\title{
CTD Observations on the North Brazil Shelf During A Multidisciplinary Amazon Shelf SEDiment Study, AMASSEDS, August 1989
}

\author{
by \\ Richard Limeburner and Robert C. Beardsley \\ Woods Hole Oceanographic Institution \\ Woods Hole, Massachusetts 02543
}

November 1989

\section{Technical Report}

\author{
Funding was provided by the National Science Foundation \\ through Grant Number OCE 88-12917 \\ Reproduction in whole or in part is permitted for any purpose of the \\ United States Government. This report should be cited as: \\ Woods Hole Oceanog. Inst. Tech. Rept., WHOI-89-53. \\ Approved for publication; distribution unlimited.
}

Approved for Distribution:

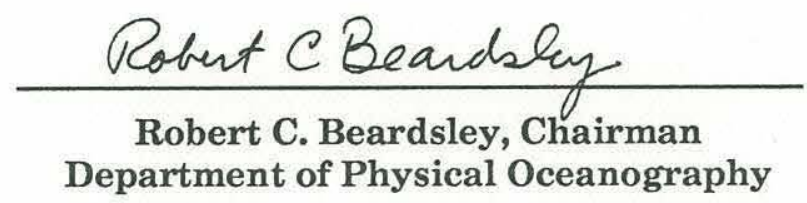




\title{
CTD OBSERVATIONS ON THE NORTH BRAZIL SHELF DURING A MULTIDISCIPLINARY AMAZON SHELF SEDIMENT STUDY, AMASSEDS, AUGUST 1989
}

\begin{abstract}
CTD and acoustic Doppler current profiler (ADCP) observations were made on the North Brazil shelf adjacent to the mouth of the Amazon River during R/V Iselin cruise I8909 August 3-14, 1989 as part of A Multidisciplinary Amazon Shelf SEDiment Study (AMASSEDS). These observations were obtained during a large-scale survey in support of geological and geochemical sampling, an anchored time series station consisting of 26 hourly CTD casts, and one transect which was repeated off the mouth of the Amazon River. The maximum sampling depth at each station was within two meters of the bottom.

The primary objectives of the AMASSEDS hydrographic measurement program were to (a) observe and characterize the temperature, salinity, density, oxygen, fluorescence and light transmission fields and their spatial variability on the north Brazilian shelf directly influenced by the Amazon River discharge, (b) resolve the seaward extent and vertical structure of the surface plume of low salinity Amazon River water during different stages of river discharge, (c) describe the spatial structure of the turbidity and associated suspended sediment distributions across the shelf, (d) characterize the properties of the Amazon shelf water beneath the surface plume and their seasonal variability, and (e) describe the landward penetration of the North Brazil Current (NBC) with respect to water properties and shelf currents. This report represents a summary in graphic and tabular form of the hydrographic observations made during the first AMASSEDS cruise (I8909) on the R/V Iselin.
\end{abstract}




\section{Table of Contents}

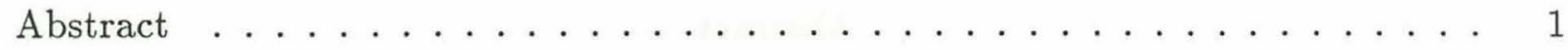

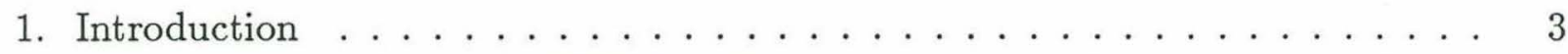

2. Instrumentation and Calibration $\ldots \ldots \ldots \ldots \ldots$

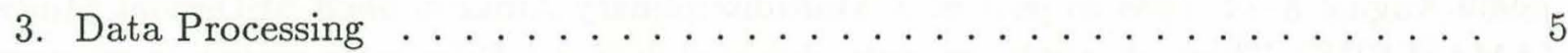

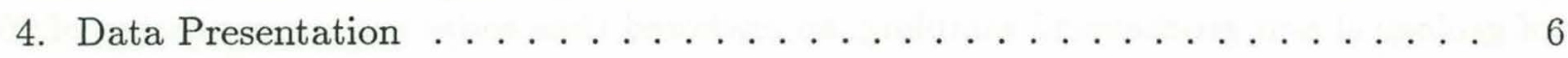

5. Acknowledgments . . . . . . . . . . . . . . . 6

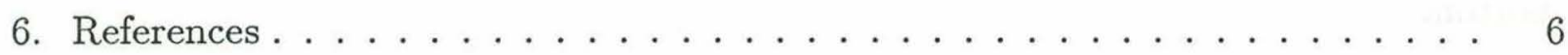

7. Graphic Description of CTD Data . . . . . . . . . . . . . 9

A. Large-Scale Survey - August 4-10, 1989 - Stations 1 to $52 \ldots \ldots$

1. Station Locations . . . . . . . . . . . . . . 10

2. Section Locations . . . . . . . . . . . . . . . . 11

3. $\mathrm{T} / \mathrm{S}$ and Vertical Sections $\ldots \ldots \ldots \ldots \ldots \ldots$

4. Horizontal Sections . . . . . . . . . . . . . . . 26

B. Time Series Anchor Station - August 11-12, 1989 - Stations 54 to 79 . 35

1. Anchor Station (53) and Time Series Station (54) Locations . . . 37

2. $\mathrm{T} / \mathrm{S}$ and Temporal Vertical Sections . . . . . . . . . 38

C. Section C - August $12-13,1989$ - Stations 80 to $86 \ldots$. . . . . . . 41

1. Station and Section Location . . . . . . . . . . . . 43

2. $\quad \mathrm{T} / \mathrm{S}$ and Vertical Sections $\ldots \ldots \ldots \ldots . \ldots . \ldots 44$

8. Station Listings and Profiles $\ldots \ldots \ldots \ldots$. . . . . . . . . 47 


\section{Introduction}

A Multidisciplinary Amazon Shelf SEDiment Study (AMASSEDS) is an multiinstitutional international program to investigate physical, geological, geochemical and biological processes within the Amazon dispersal system. The primary objective of AMASSEDS is to understand the sedimentary processes on the continental shelf near the mouth of the Amazon River. Previous studies of the Amazon shelf and similar environments suggest that the enormous suspended sediment load carried over the inner shelf in the local water column strongly influences other factors such as seabed morphology and biological productivity, and leads to creation of a subaqueous delta. The physical oceanographic component of AMASSEDS is directed toward understanding circulation and boundary layer processes on the Amazon shelf. The primary objectives are to (a) measure and characterize the flow and mixing processes in the low salinity frontal zone, (b) to estimate the contributions to bottom stress from subtidal motions, tidal flow, and surface waves, and (c) to quantify the cross-shelf advective and dispersive transport mechanisms. The physical oceanographic field work includes: short-term (February May, 1990) moored measurements of wind, current, temperature, conductivity, and surface waves to determine tidal and low-frequency variability in the shelf flow field, density stratification, and surface and bottom stresses; a series of four hydrographic surveys to map the distributions of temperature, salinity, and turbidity during different stages of river discharge; and short-term high resolution measurements of the small-scale circulation and mixing processes in the frontal zone. Satellite imagery of the sea surface is being collected to describe the synoptic distribution of suspended sediment. Finally, two satellite-tracked drifting buoys are being deployed on each of the four hydrographic surveys. The first survey August, 1989 was completed during falling river discharge; the second survey is planned for February, 1990 during rising river discharge; the third survey is planned for May, 1990 during maximum river discharge, and the fourth and last cruise is planned during November, 1991 during low river discharge These measurements include hydrographic and current profiling with a CTD and a $600 \mathrm{kHz}$ acoustic Doppler current profiler (ADCP), respectively.

We present here in graphic and tabular form a preliminary analysis of the CTD observations made on the North Brazil shelf on board the R/V Iselin during cruise I8909 August 3 - 14, 1989. A total of 86 CTD stations were made on I8909, and the following station summary is intended to provide a brief description of the sequence of CTD sampling during the cruise. Vertical and horizontal sections of water properties 
obtained from the regional CTD survey August 3-14 are shown in Section 7. Listings of the CTD observations at standard depths for all stations are given in Section 8.

\section{CTD STATION LISTING SUMMARY}

Leg 1 - August 3-14, 1989

Stations 1-52 - August 4-10. Initial large-scale survey of the North Brazil shelf consisting of 7 cross-shelf transects to map the initial fields of temperature, salinity, sigma-t, oxygen, fluorescence, and light transmission.

Station 53 - August 10. CTD cast made just after deployment of a pilot mooring to test depth penetration of the anchor into the sediments.

Stations 54-79 - August 11-12. 26 hour time series anchor station consisting of hourly CTD casts.

Stations 81-86 - August 12-13. Repeat of Section C adjacent to the mouth of the Amazon River.

\section{Instrumentation and Calibration}

A Neil Brown Instrument Systems (NBIS) model MKIII CTD fish was used as the primary profiling instrument during R/V Iselin cruise I8909. The instrument provided continuous sampling of temperature, conductivity, pressure, and light transmission. Salinity and density were subsequently derived from the measured variables. The oxygen and fluorescence sensors on the underwater unit did not work properly during the entire cruise. The instrument package consisted of the MKIII CTD underwater unit mounted one meter below a General Oceanics rosette sampler with 12 five-liter Niskin bottles. A Sea Tech $5 \mathrm{~cm}$ pathlength transmissometer was mounted near the CTD fish. The fast response thermistor on the CTD fish was disabled and the instrument sampled at a rate of $16 \mathrm{~Hz}$. Lowering speeds were approximately $36 \mathrm{~m} / \mathrm{min}$ to match the response times of the temperature and conductivity sensors (Giles and McDougall, 1986). The pressure sensor had a full range of $6400 \mathrm{db}$. 
The CTD underwater unit was part of the R/V Iselin's scientific equipment. Water samples were normally collected at each station on the up cast at a depth where the vertical stratification and suspended sediment concentration were minimal. The calibration samples were then processed onboard during the cruise using a Guildline AutoSal salinometer to determine salinity. The individual salinities were converted to in situ conductivity and compared to the conductivity output of the instrument. A least-squares fit of the difference between the bottle and instrument conductivity gives

$$
C(B O T T L E)-C(C T D)=-0.0000349498 * X-0.0176786
$$

where $X$ is the station number which varied from 1 to 86 , and the sum of the squares of the residuals is $0.0121 \mathrm{mmho}^{2}$. This means that the CTD conductivity sensor drift and offset were small during the cruise and that the rms difference between the corrected CTD and bottle conductivity values was \pm 0.004 mmho.

The Sea Tech transmissometer data is presented as a percent of full scale. The primary light transmission sensor (channel b) functioned intermittently at stations 24 - 29. At station 30 a secondary $5 \mathrm{~cm}$ pathlength transmissionmeter was installed on channel $\mathrm{b}$, and the primary sensor was placed on channel a for observation and testing. Stations 24-29 were located in relatively clear water and a linear interpolation was made for all bad light transmission data.

\section{Data Processing}

The CTD data were recorded at sea with a PC-based data acquisition system written by Howard Saklad of the University of Alaska, Institute of Marine Science. The program acquires the raw CTD data at $16 \mathrm{~Hz}$, displays the data in real time, postcast data averages, and prints lists of data at each station. Normally, only the down profile is processed at sea. During post cruise data processing, the conductivity calibration was first applied to the raw data. Then an exponential recursive filter (Middleton and Foster, 1980) described by Cox was applied to pressure and conductivity to match the amplitude and phase of the platinum temperature sensor. The Cox filter has the form

$$
c^{\prime}(t)=w_{0} c^{\prime}(t-d t)+\left(1-w_{0}\right) c(t),
$$


where $c^{\prime}(t)$ is the filtered conductivity at time $t, c^{\prime}(t-d t)$ is the previous filtered conductivity, and $c(t)$ is the original unfiltered conductivity at time $t$. The filter weight is given by

$$
w_{0}=e^{-d t / t l a g}
$$

where $d t=.064 \mathrm{sec}$ is the sampling period, and tlag $=.235 \mathrm{sec}$ is the time lag of the platinum temperature sensor. A uniform pressure series of $1 \mathrm{db}$ interval was then created from the filtered data, after spurious data observations (spikes) had been replaced with interpolated data. Salinity was calculated using Lewis (1980) and a value of 42.914 was used to convert to conductivity ratio (Culkin and Smith, 1980).

\section{Data Presentation}

The hydrographic data collected on cruise I8909 are shown in the form of vertical sections, T/S correlations and horizontal sections in Section 7, and vertical profiles and tabular lists at graduated depths are given in Section 8.

\section{Acknowledgments}

Cruise I8909 on the R/V Iselin was supported by NSF Grant OCE88-12917. Dr. David Demaster was the Chief Scientist on the multidisciplinary cruise. The work was a cooperative effort by R. Limeburner, Y. Yu, R. Johnson, T. Milligan, R. Pope, D. Demaster, S. Hardin, I. Dias, J. Faleiro, J. Neto, H. Vital and D. Muehe. Their assistance is greatly appreciated. Finally, the helpfulness of the officers and crew of the $\mathrm{R} / \mathrm{V}$ Iselin contributed significantly to the success of the cruise.

\section{References}

Culkin, F. and N. D. Smith (1980) Determination of the concentration of potassium chloride having the same electrical conductivity, at $15^{\circ} \mathrm{C}$ and infinite frequency, as 
standard seawater of salinity $35.000 \mathrm{ppm}$ (chlorinity $19.37394 \mathrm{ppm})$. IEEE Journal of Ocean Engineering, OE-5, 22-23.

Giles, A. B., and T. J. McDougall (1986) Two methods for the reduction of salinity spiking of CTDs. Deep-Sea Research, 33(9), 1253-1274.

Lewis, L. L. (1980) The Practical Salinity Scale 1978 and its antecedents. IEEE Journal of Ocean Engineering, OE-5, 3-8.

Middleton, J. H., and T. D. Foster (1980) Fine structure measurements in a temperature compensated halocline. Journal of Geophysical Research, 85(C2), 1107-1122. 

7. Graphic Description of CTD Data

A. Large-Scale Survey - August 4-10, 1989 Stations 1 to 52 


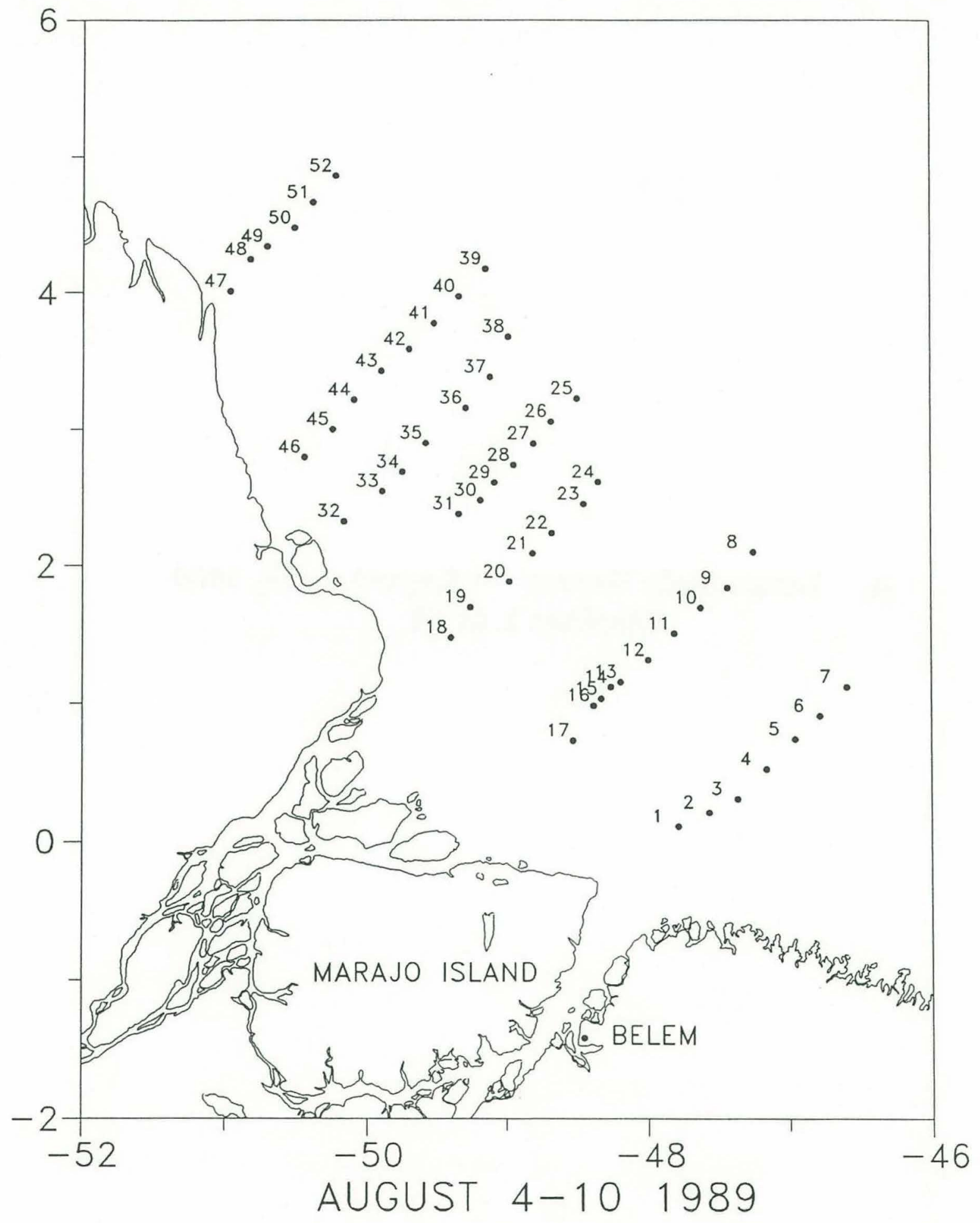




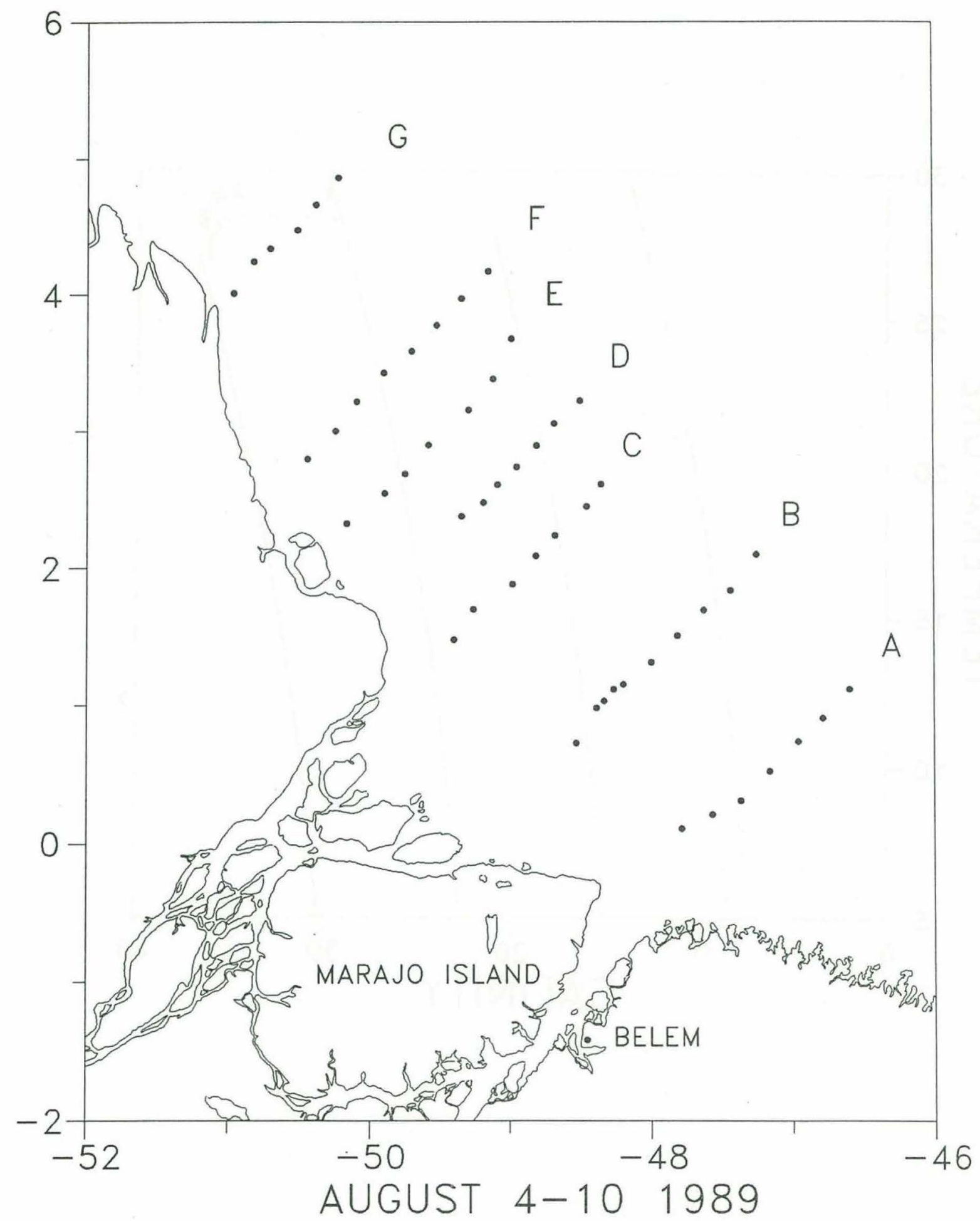




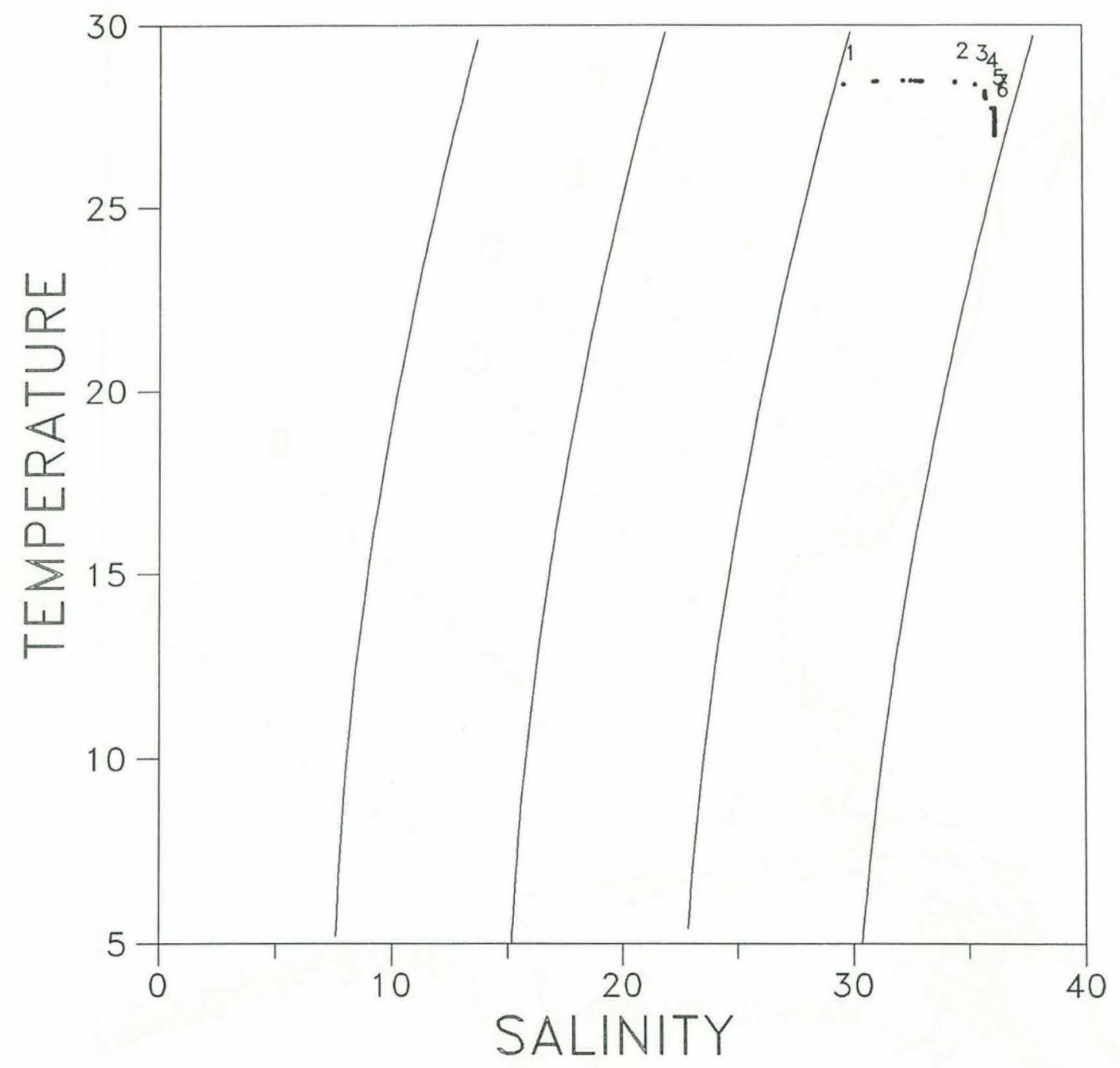



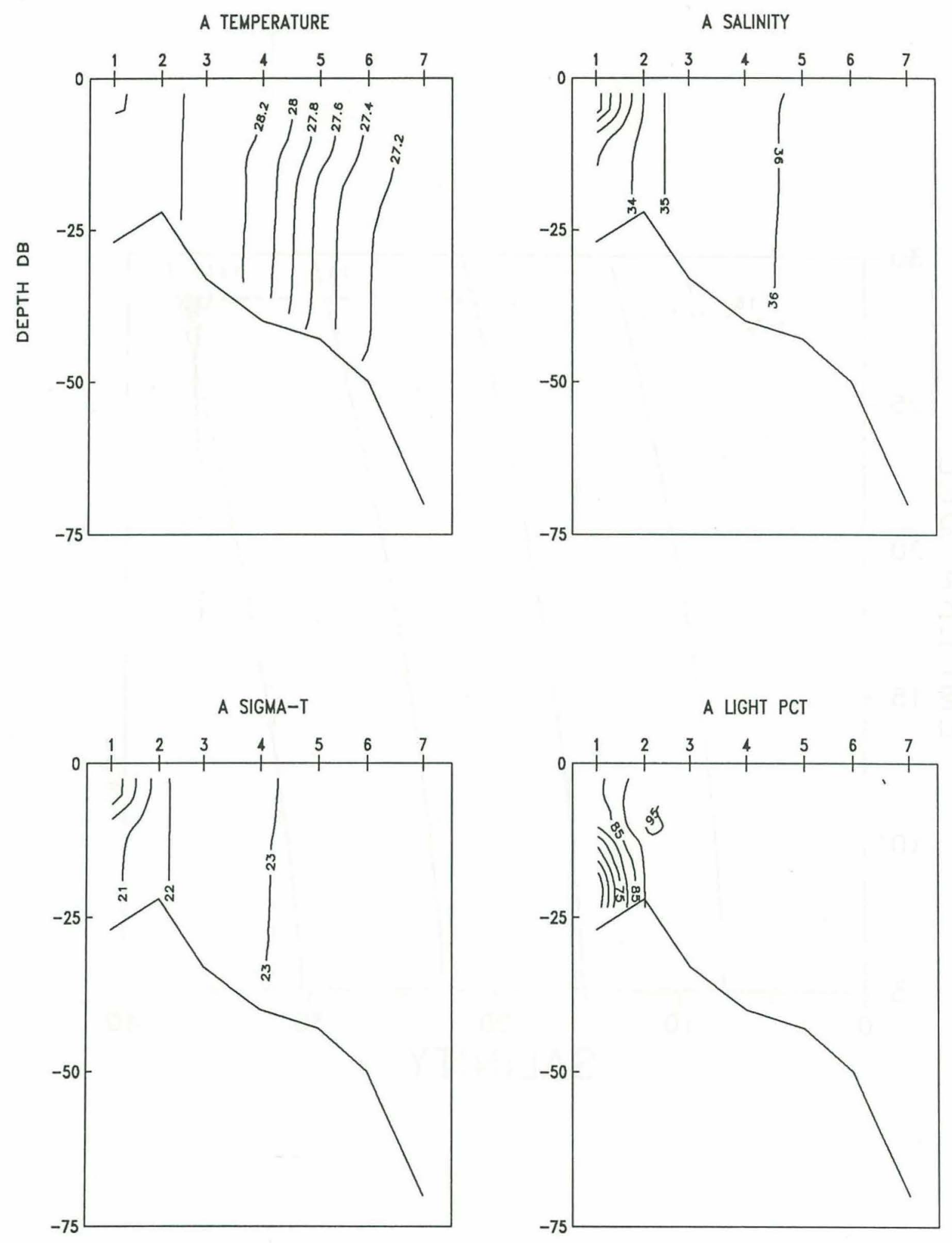


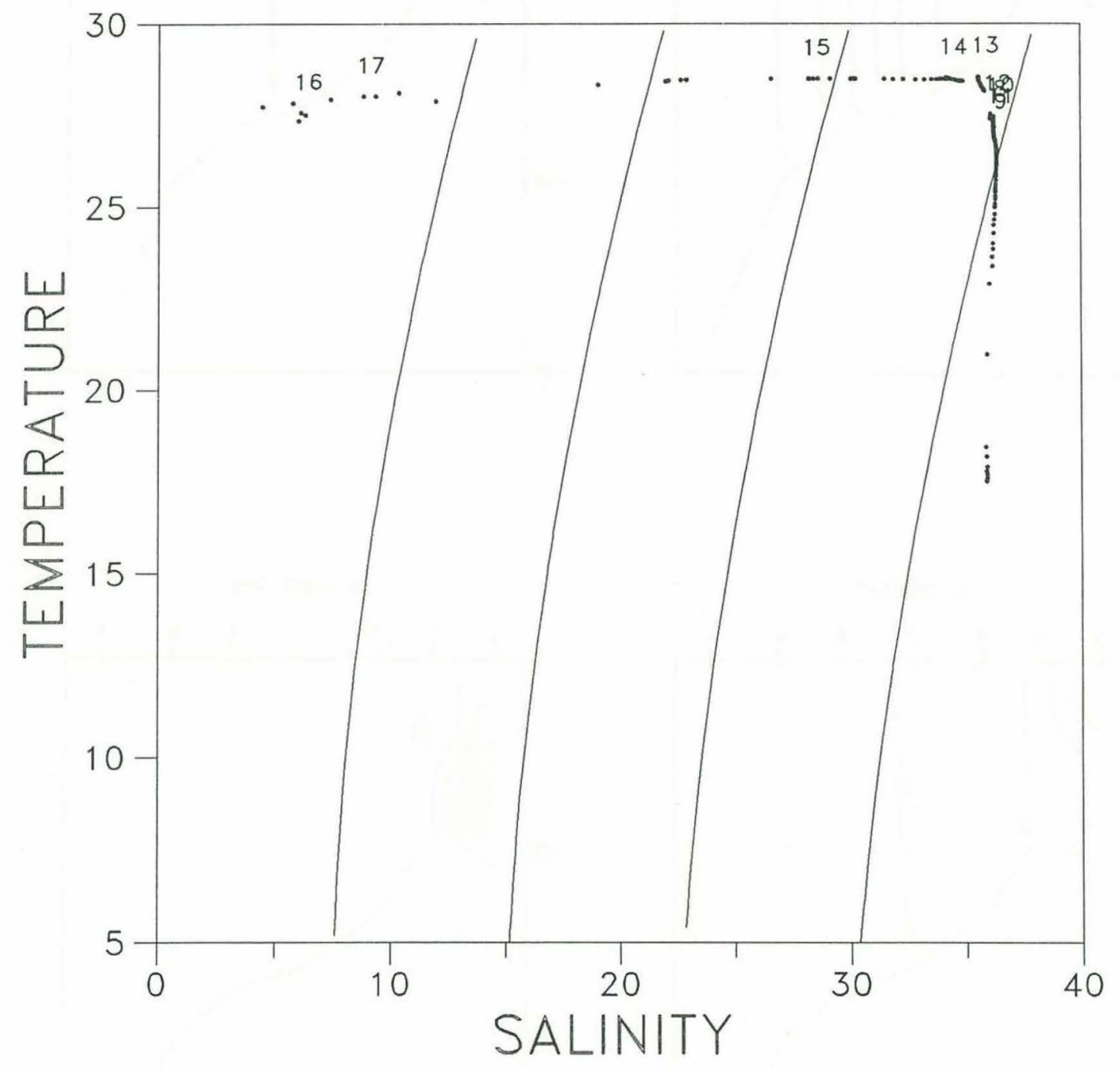



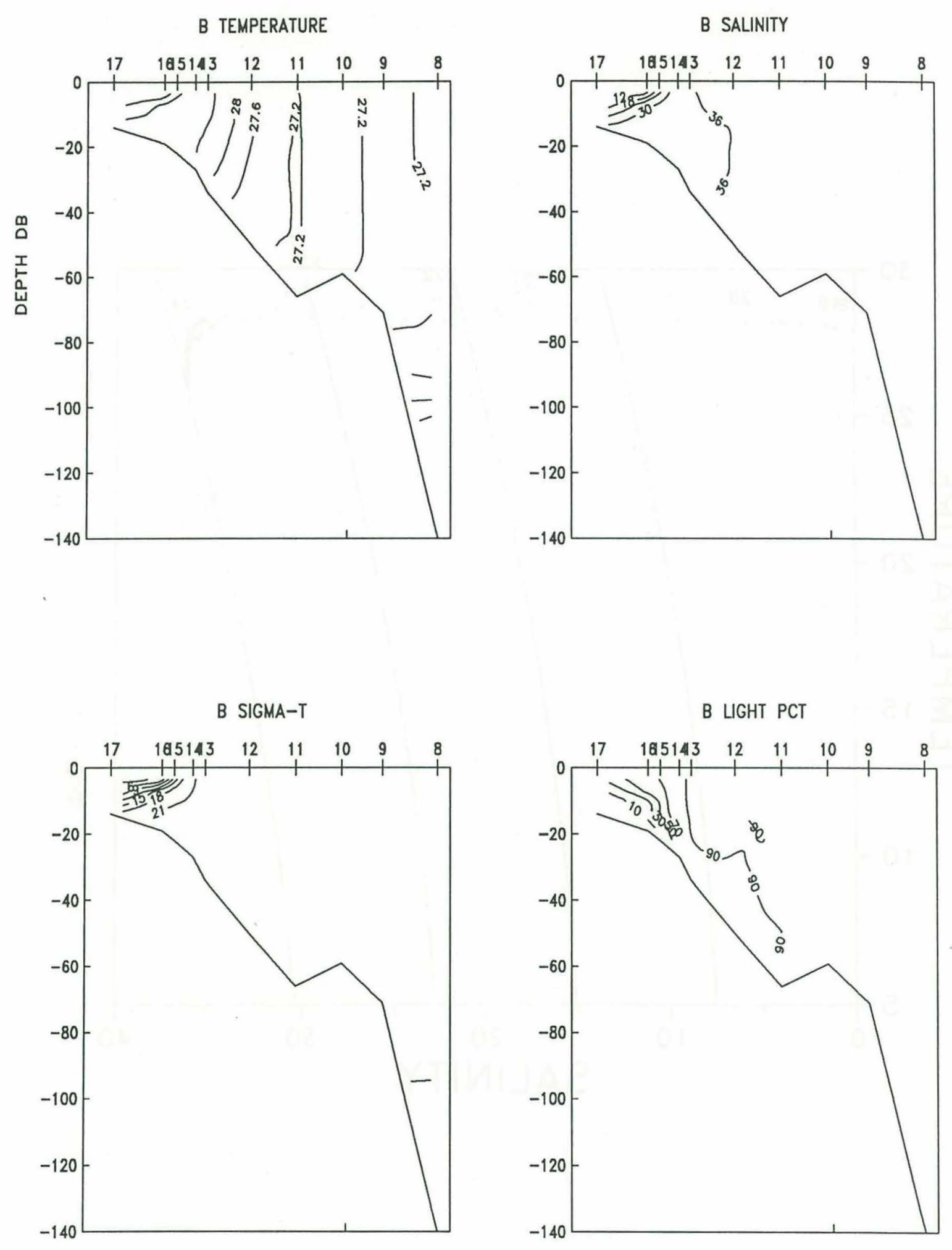


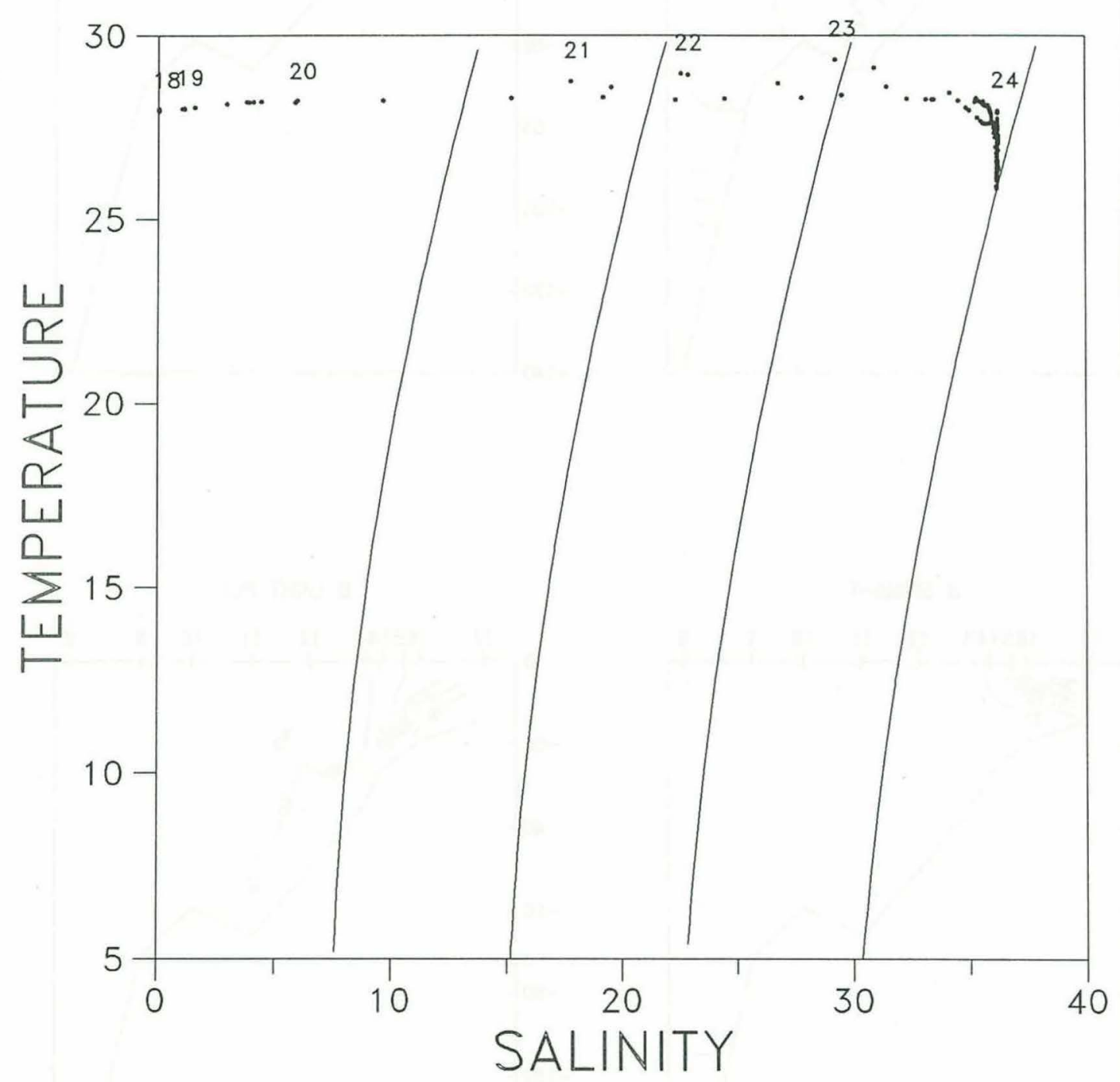



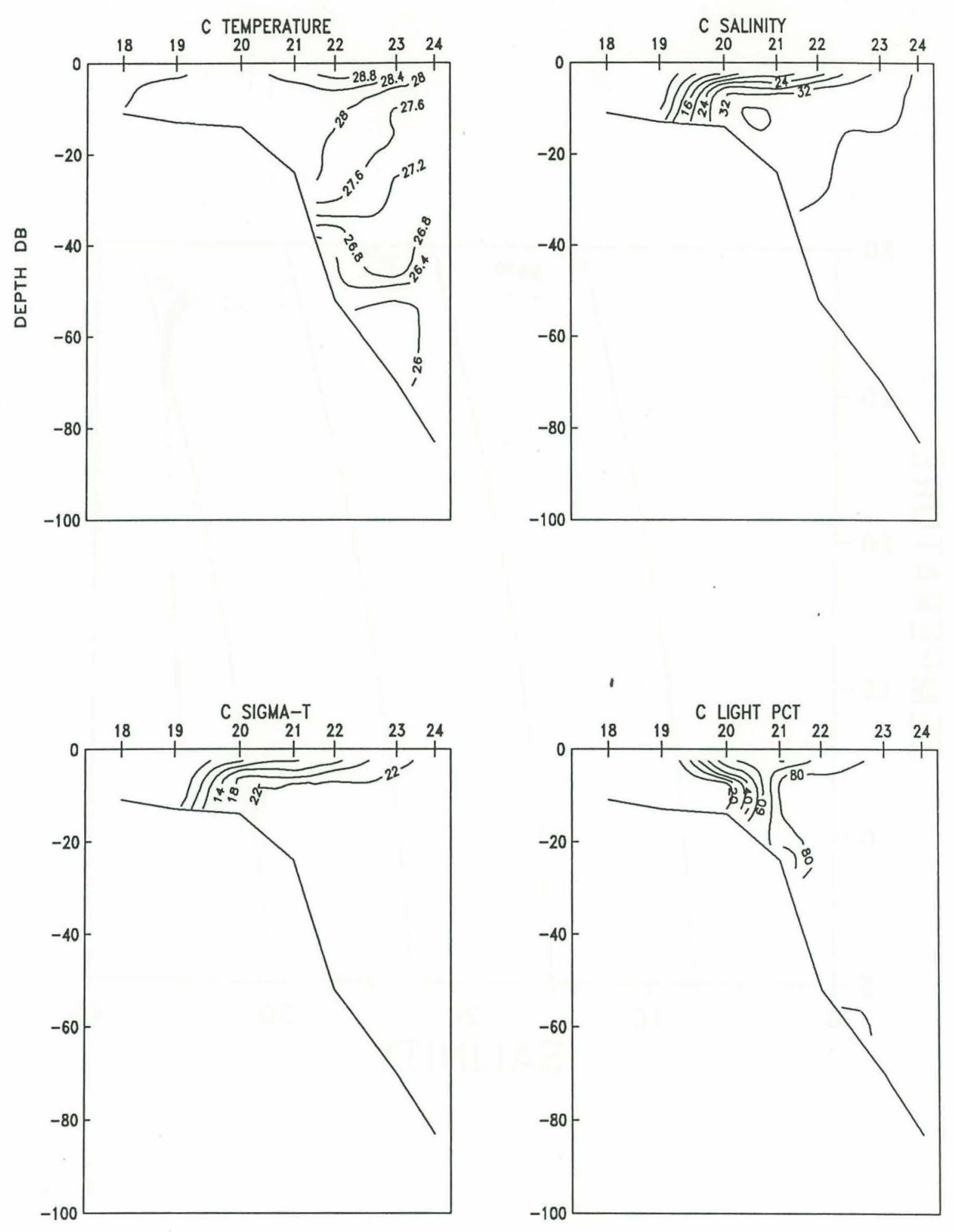


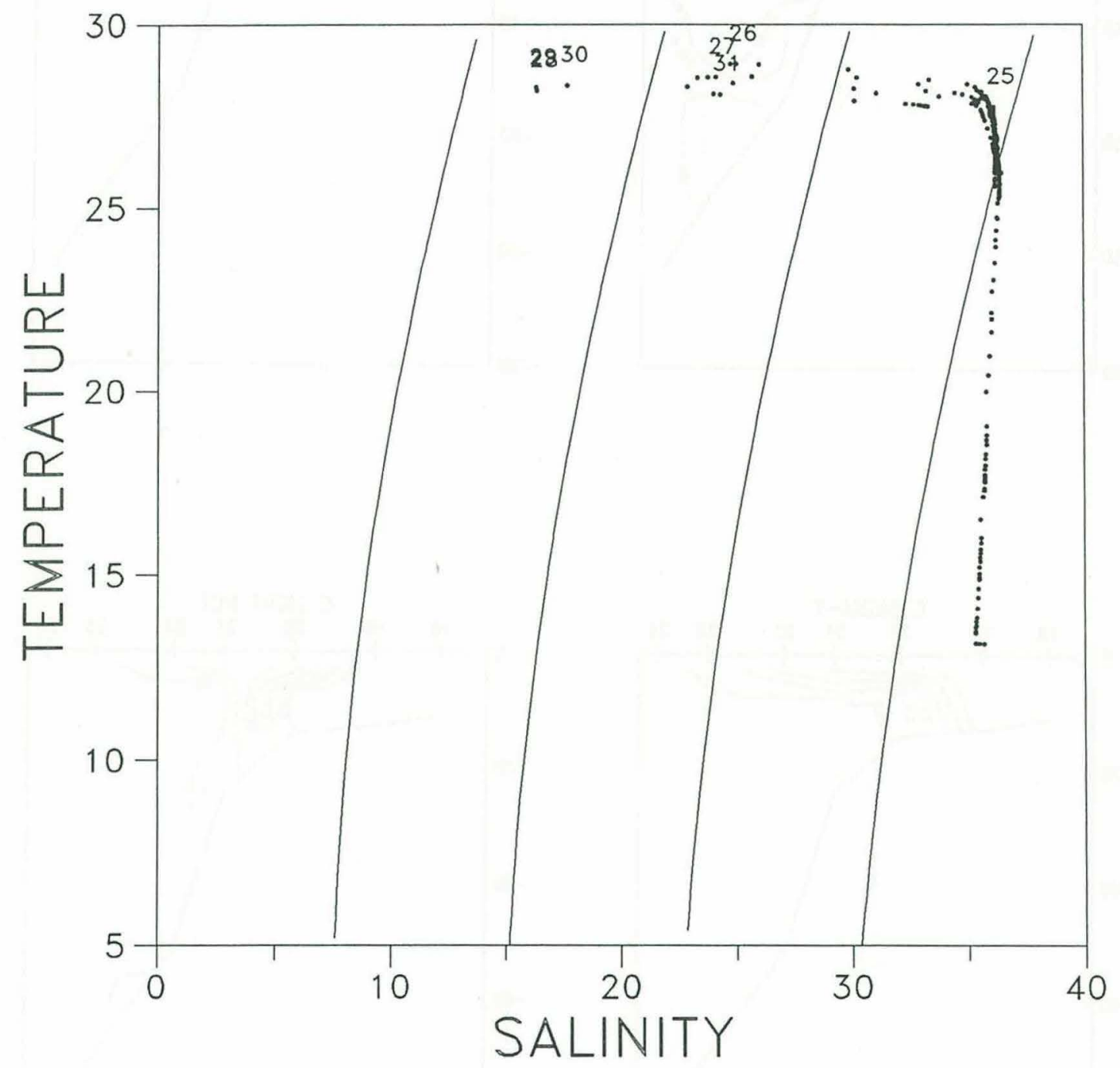



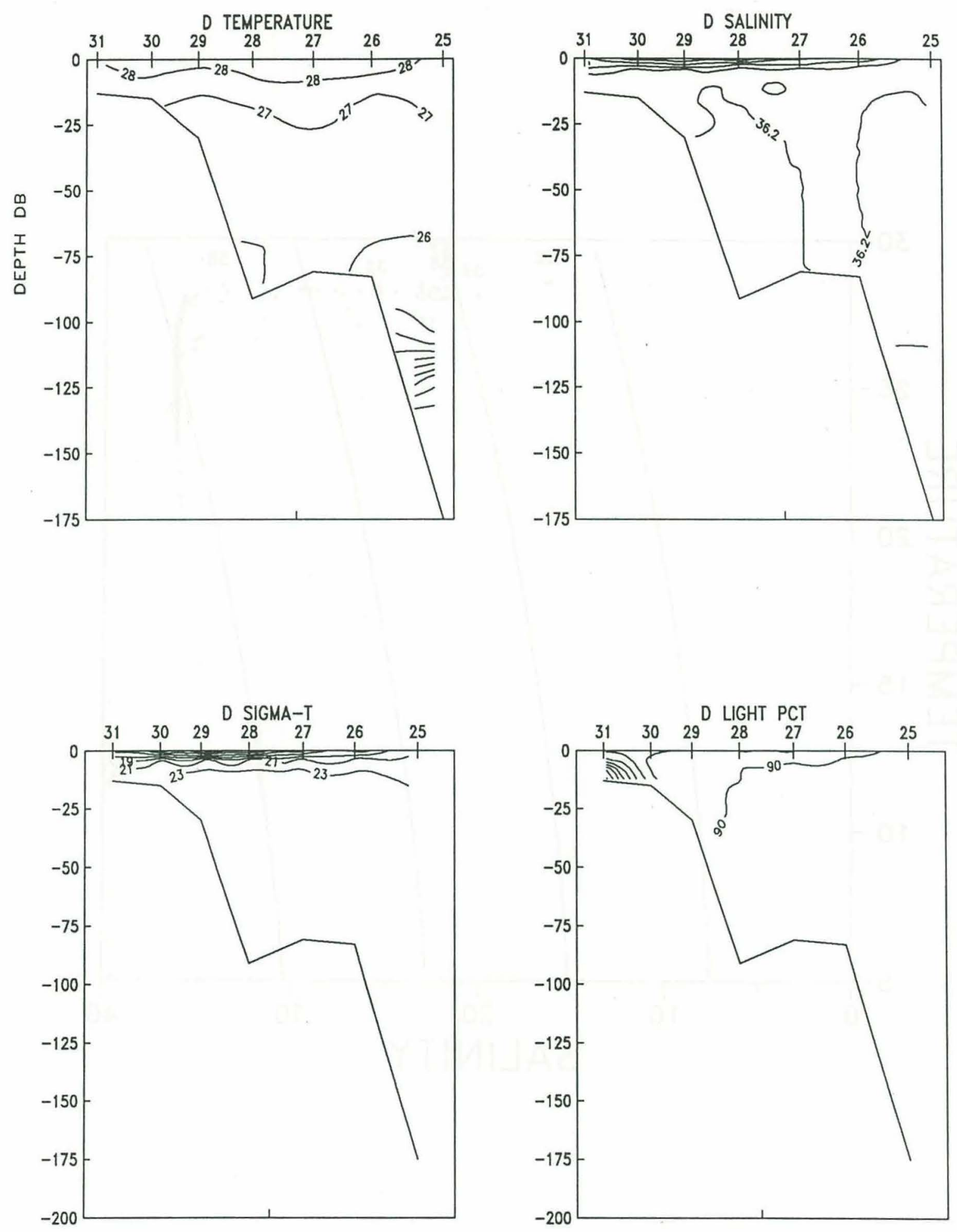


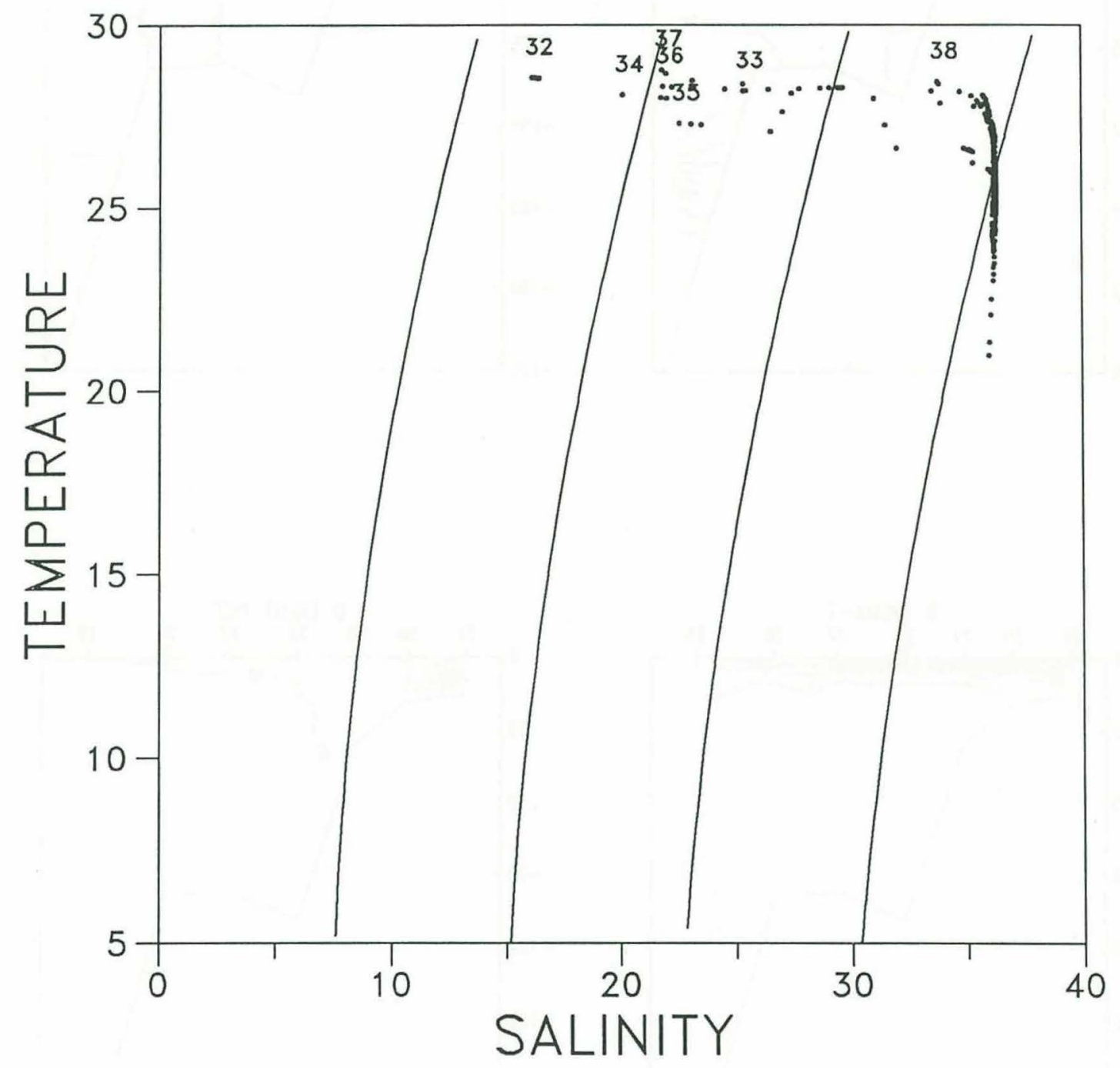



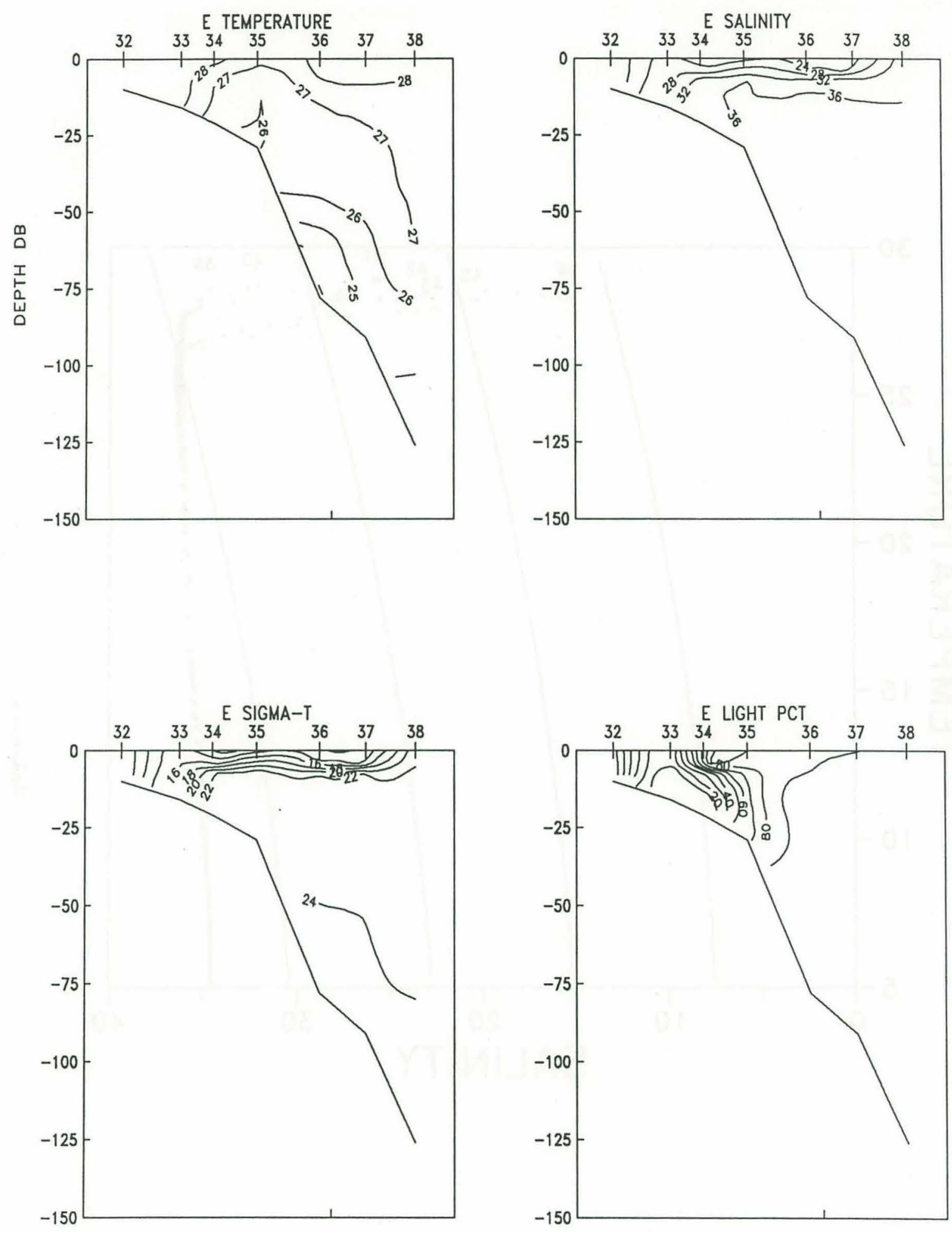


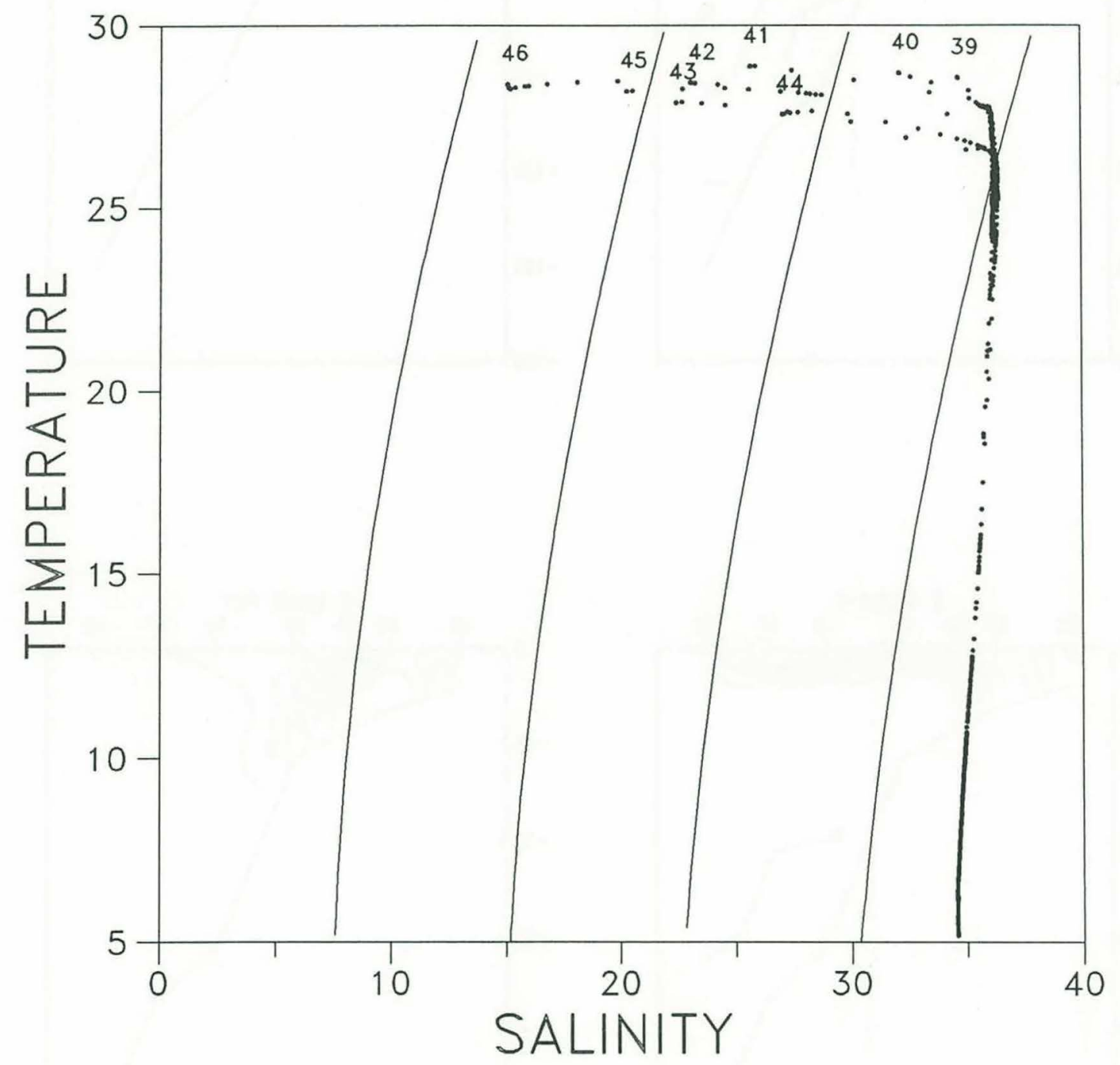



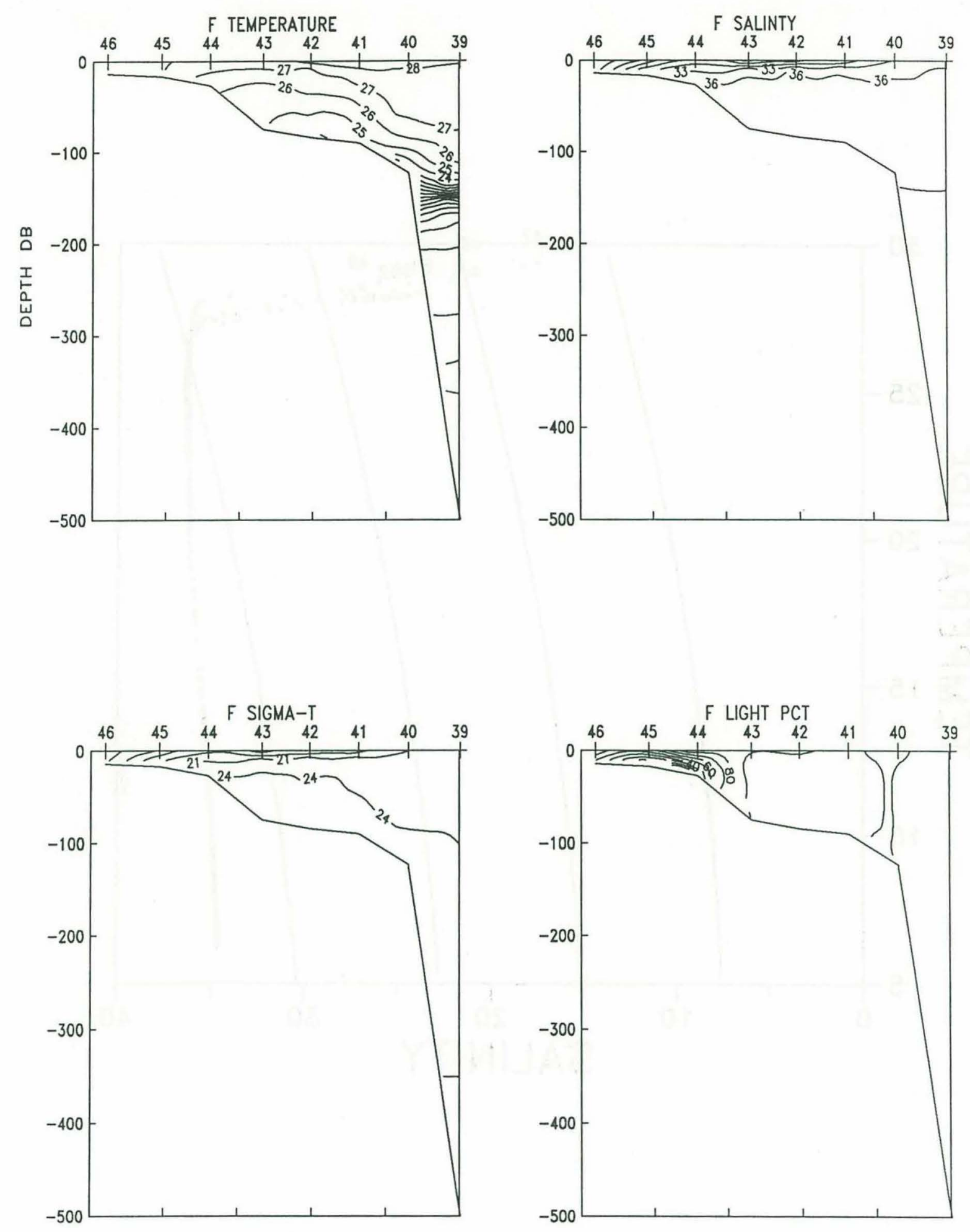


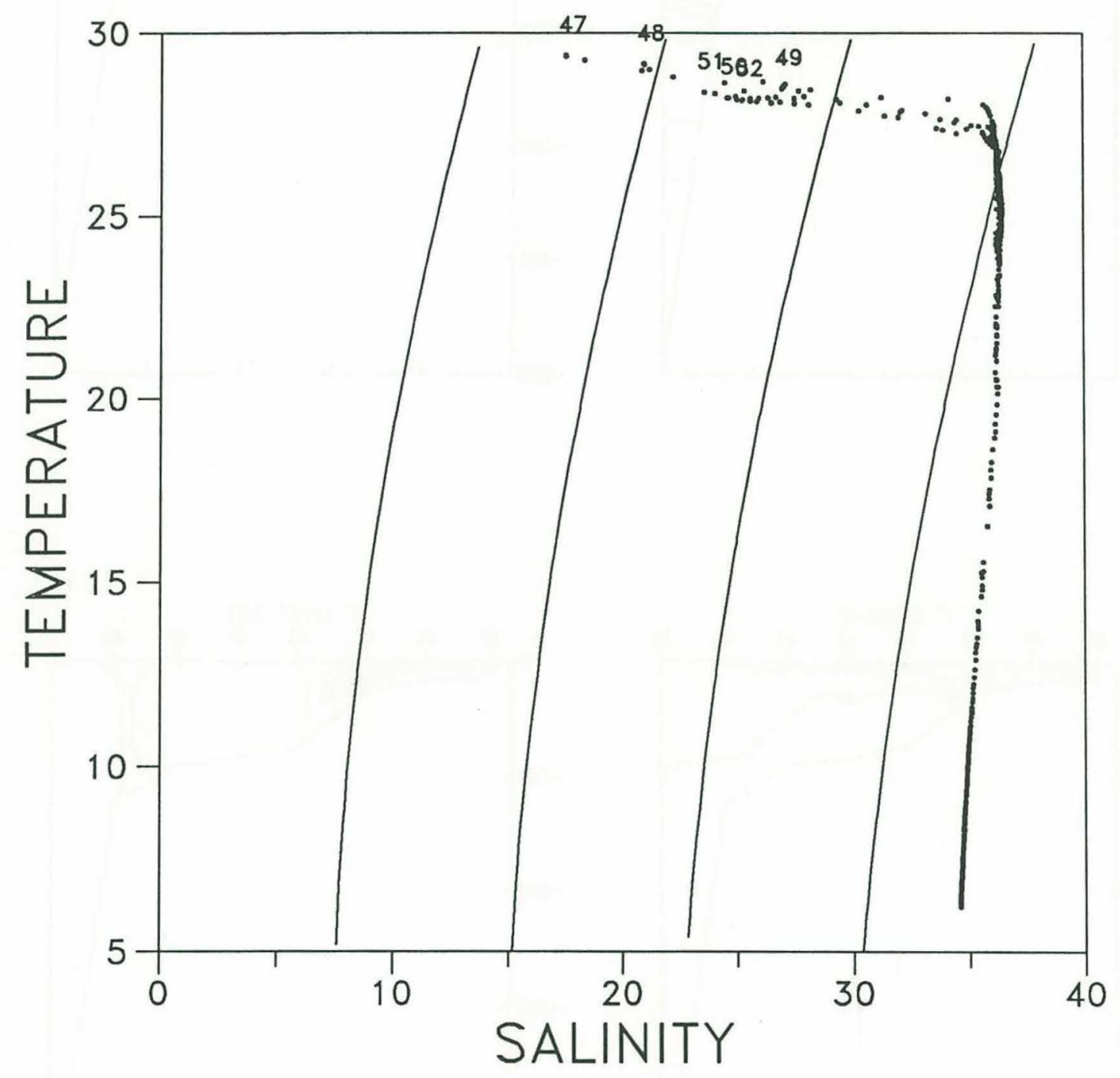



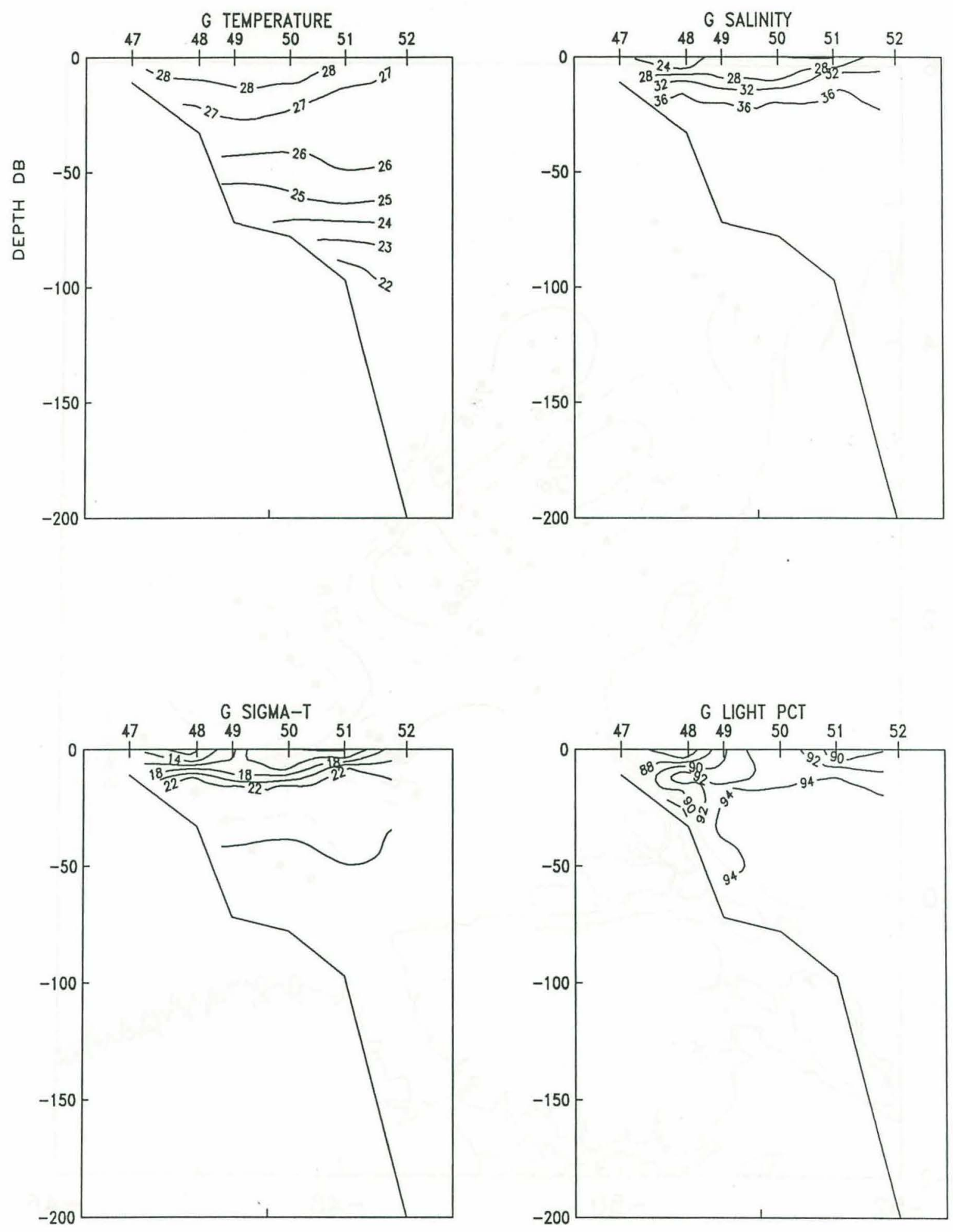


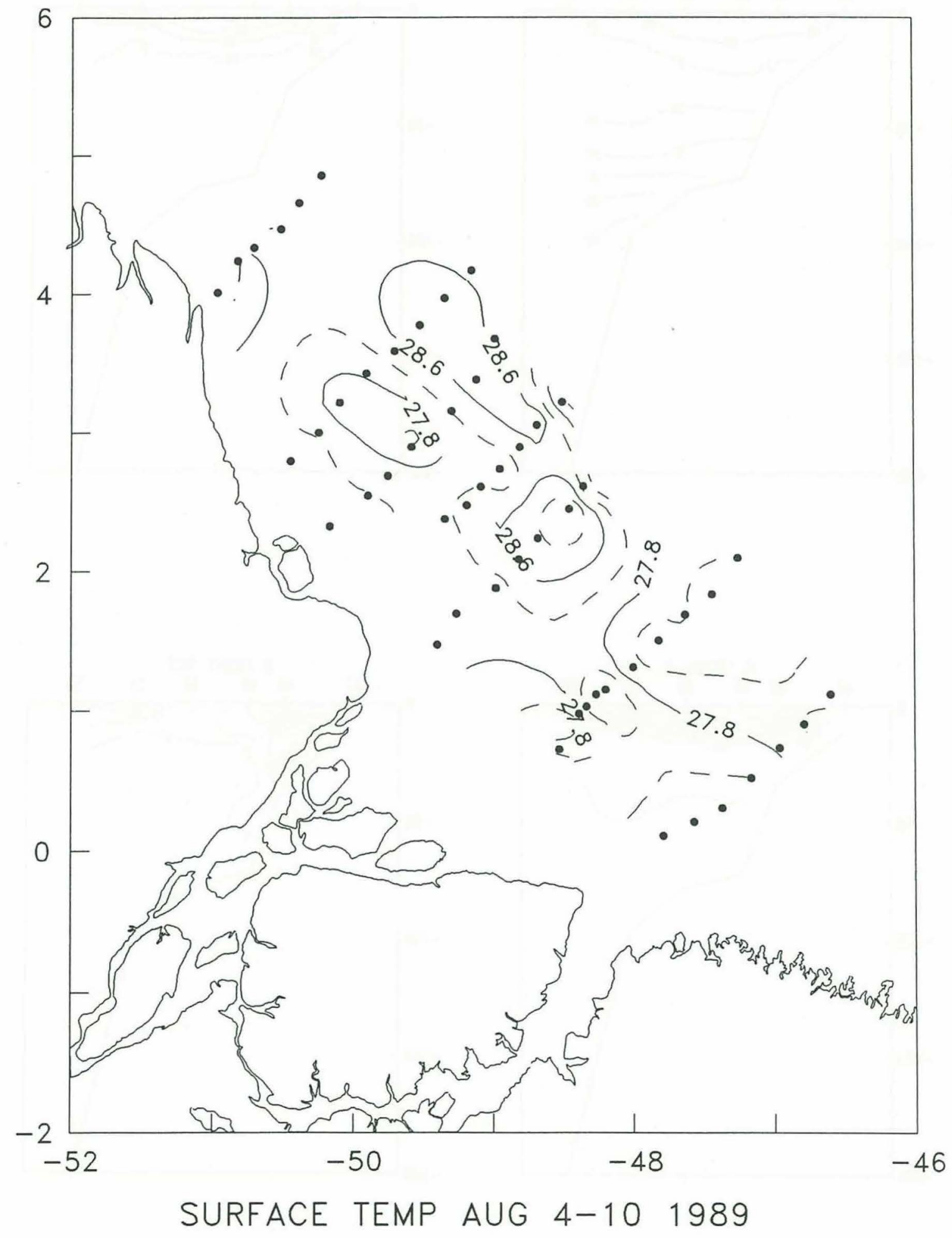




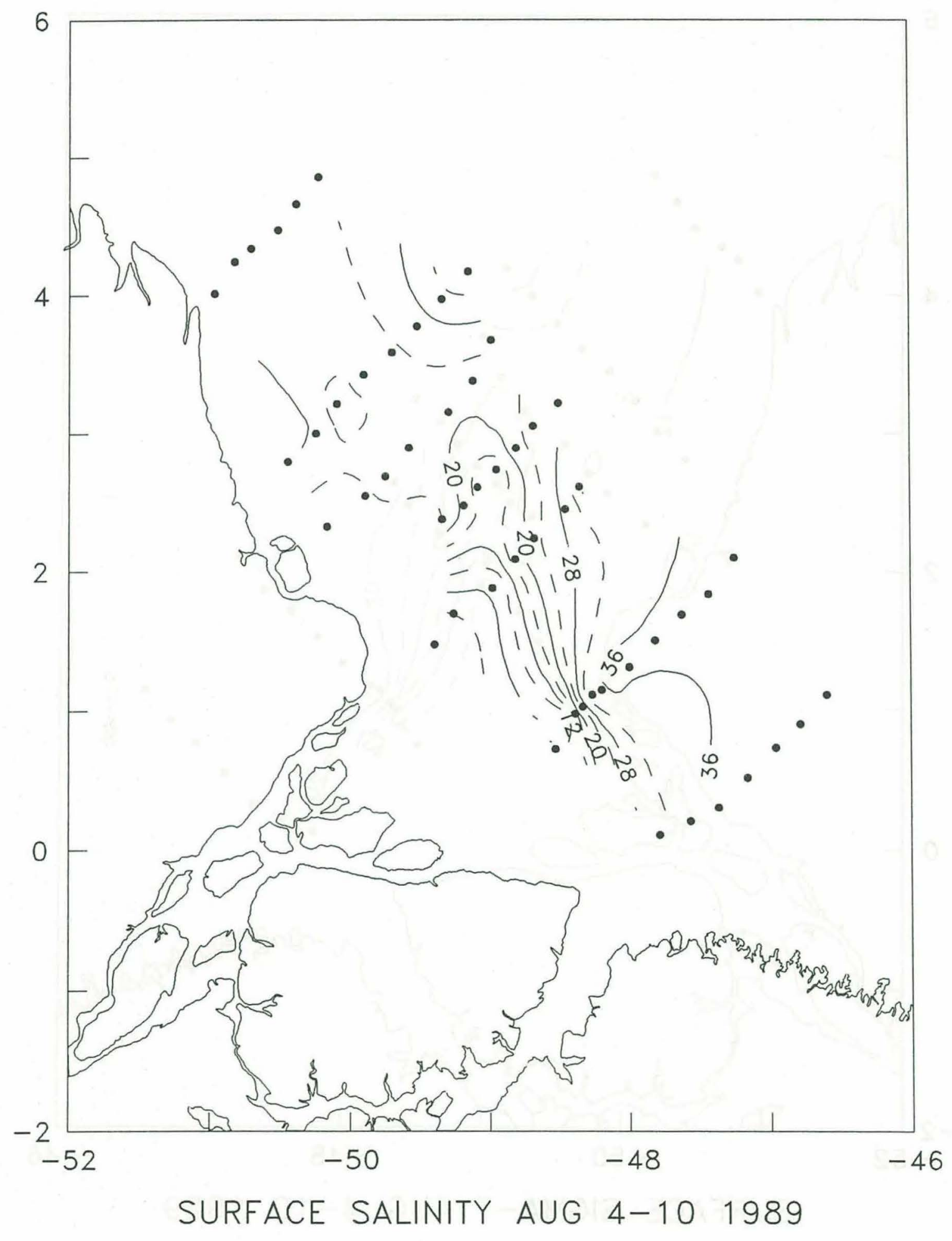




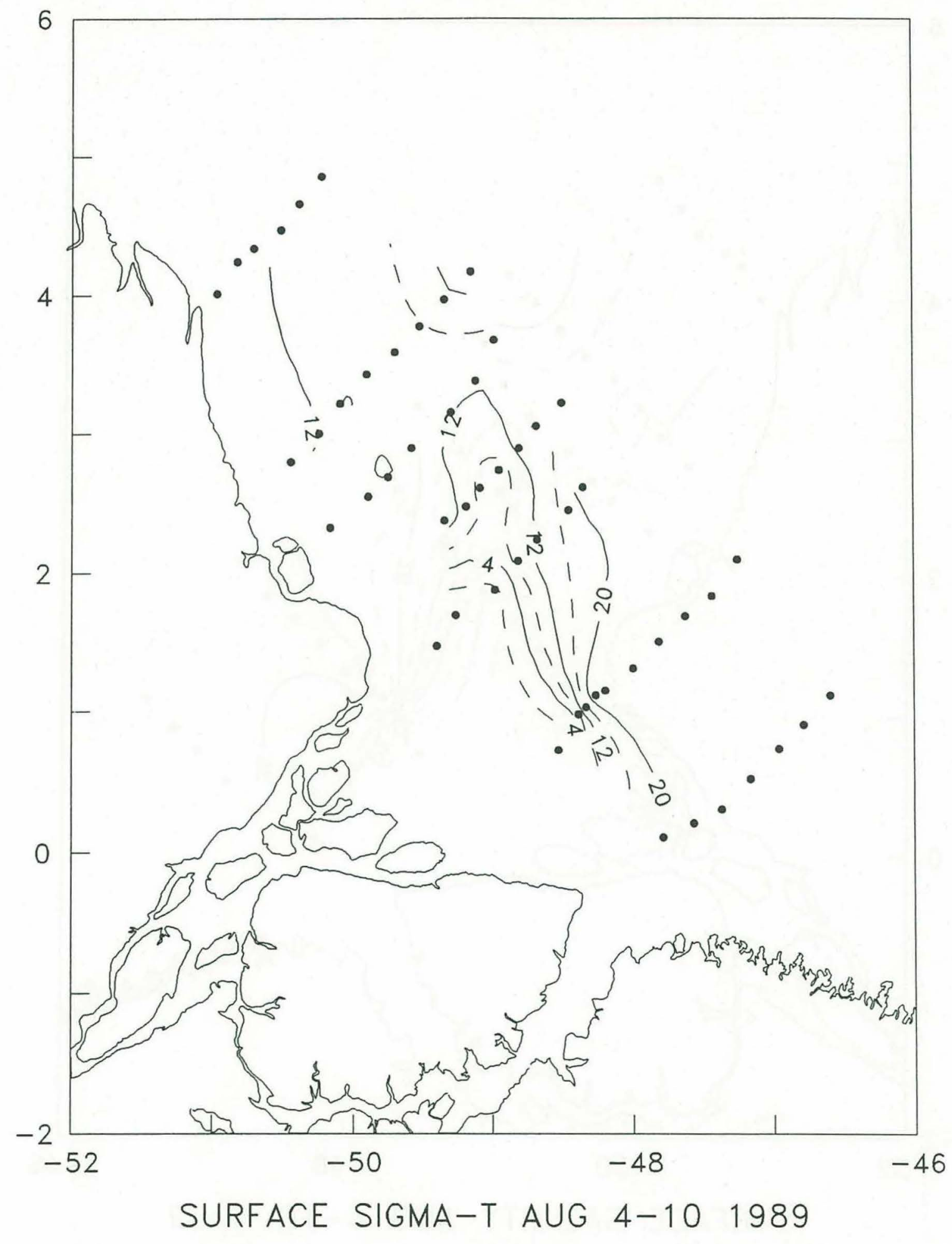




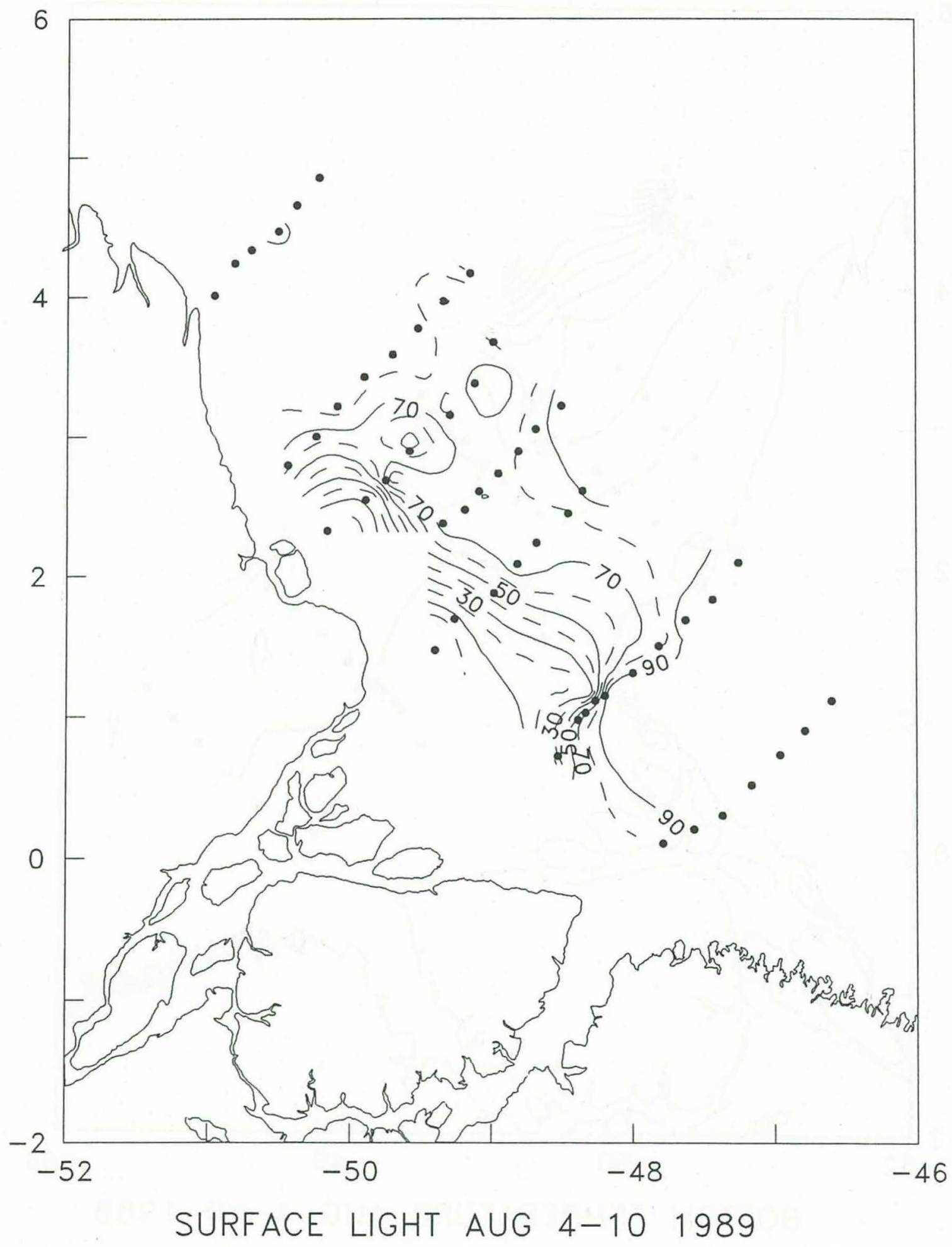




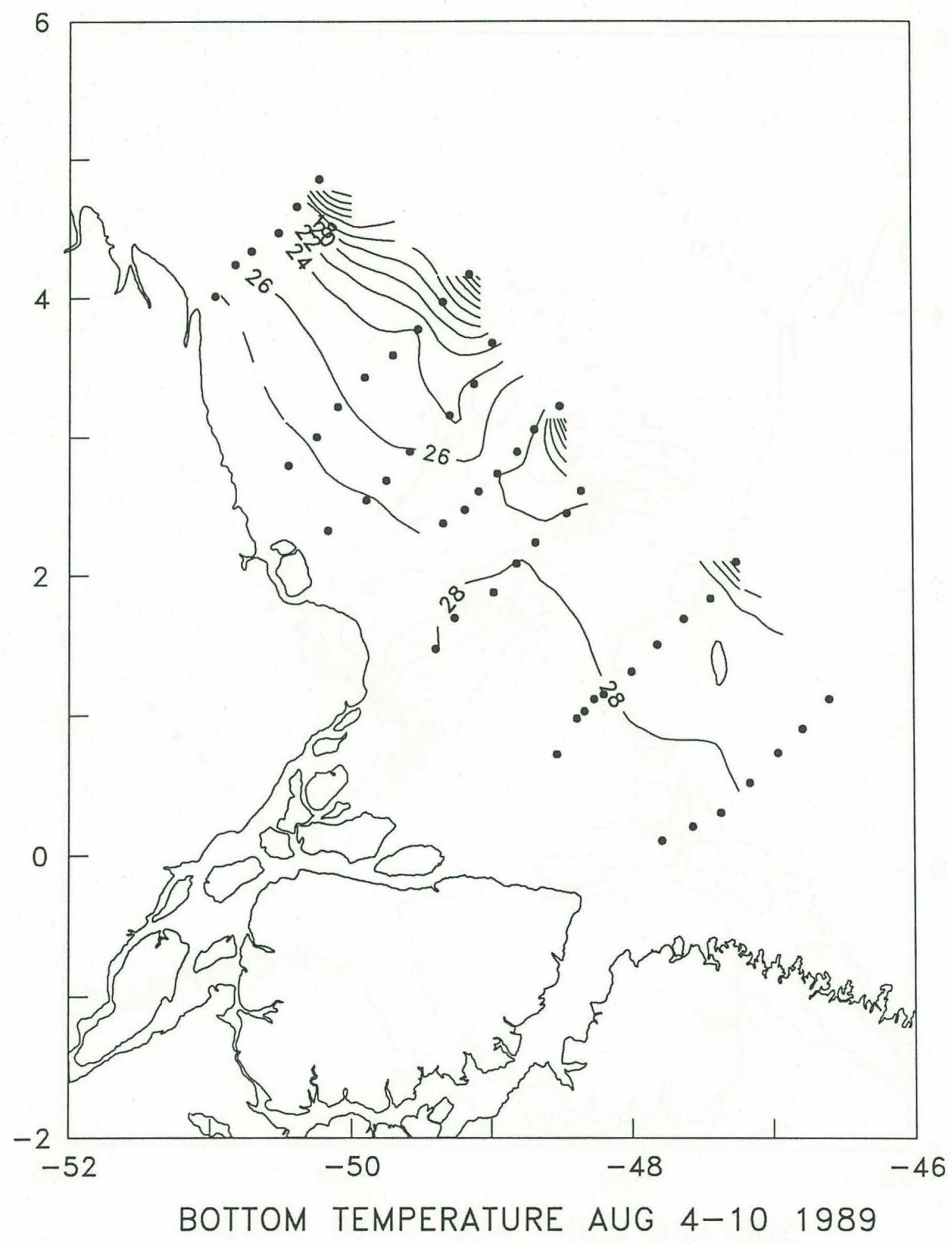




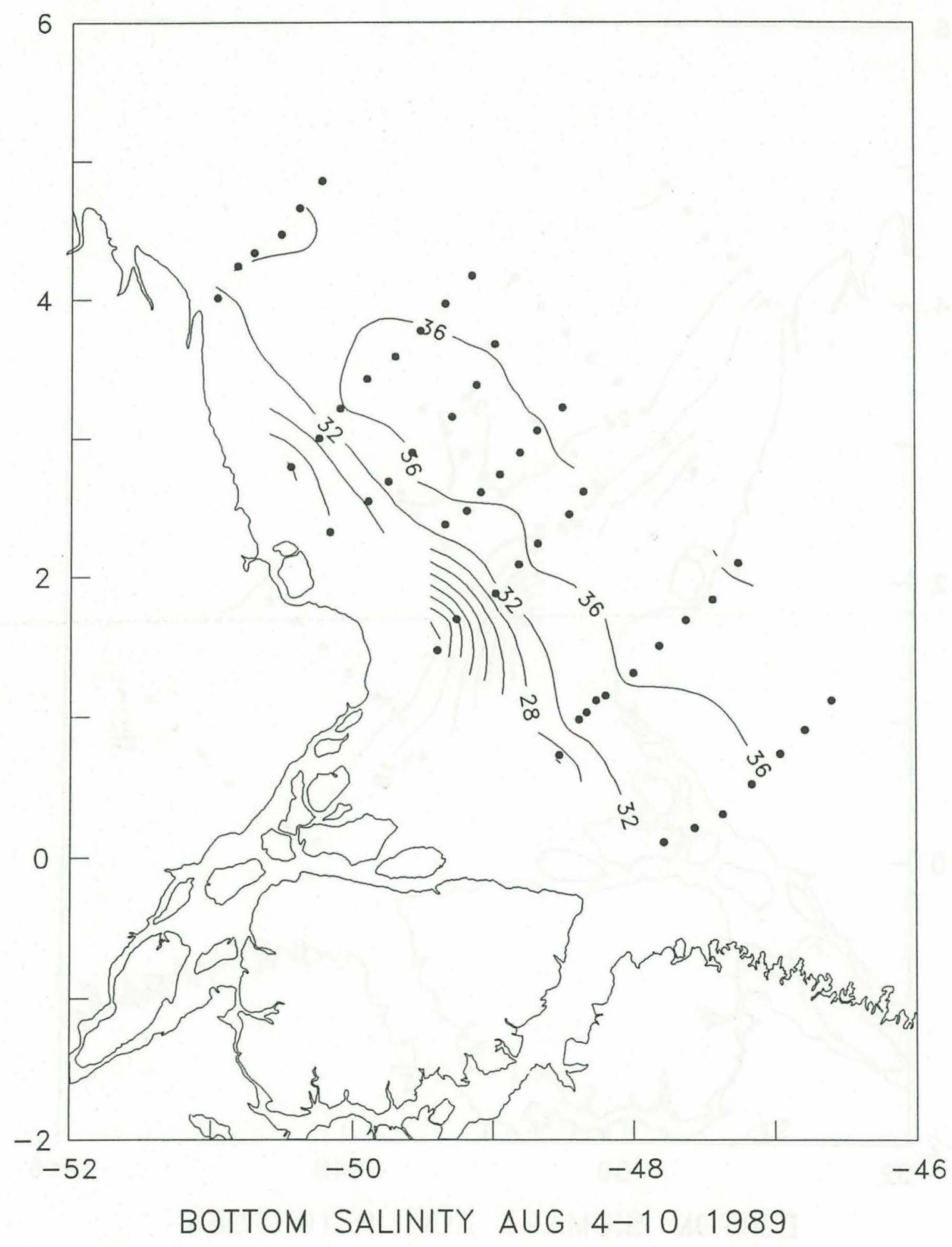




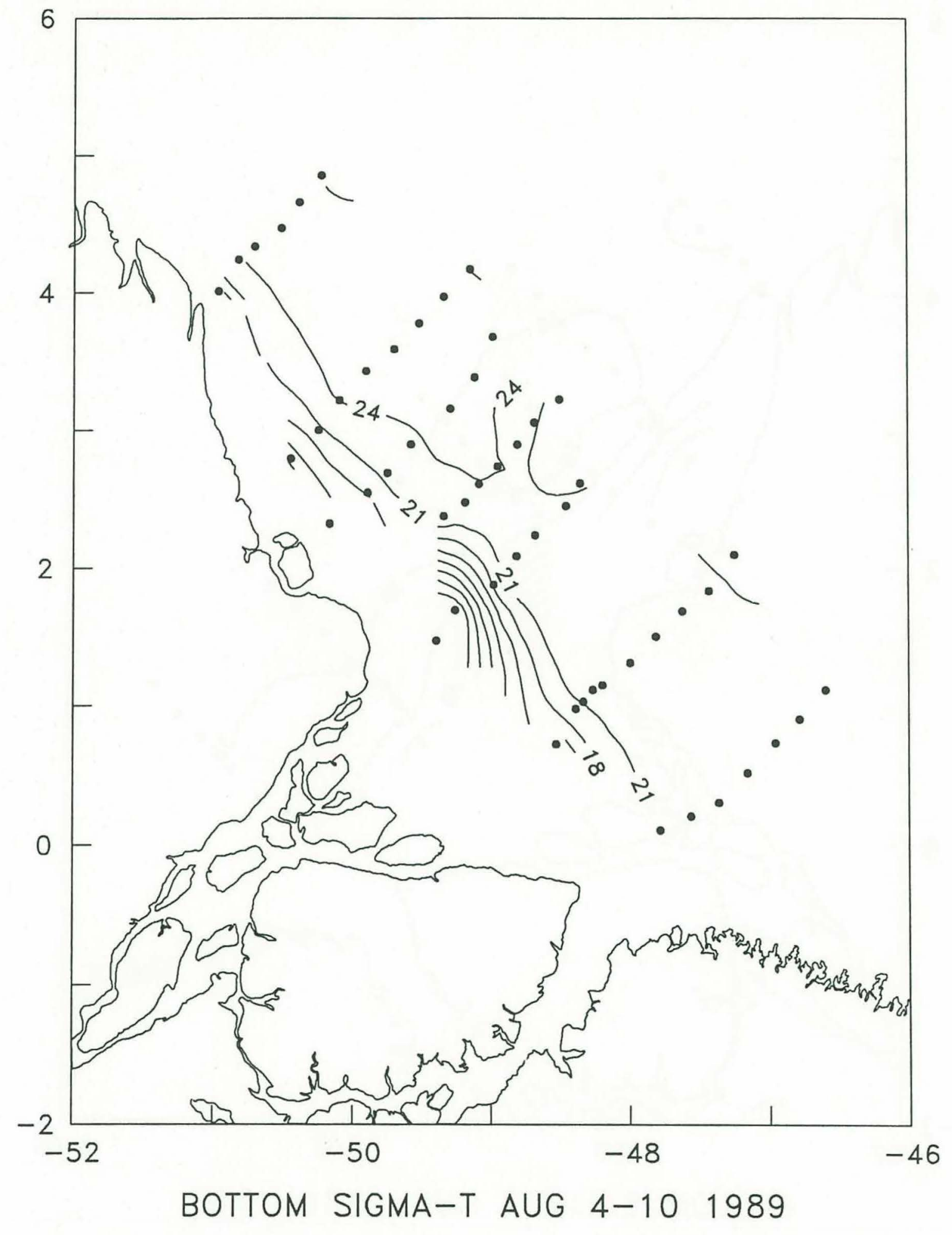




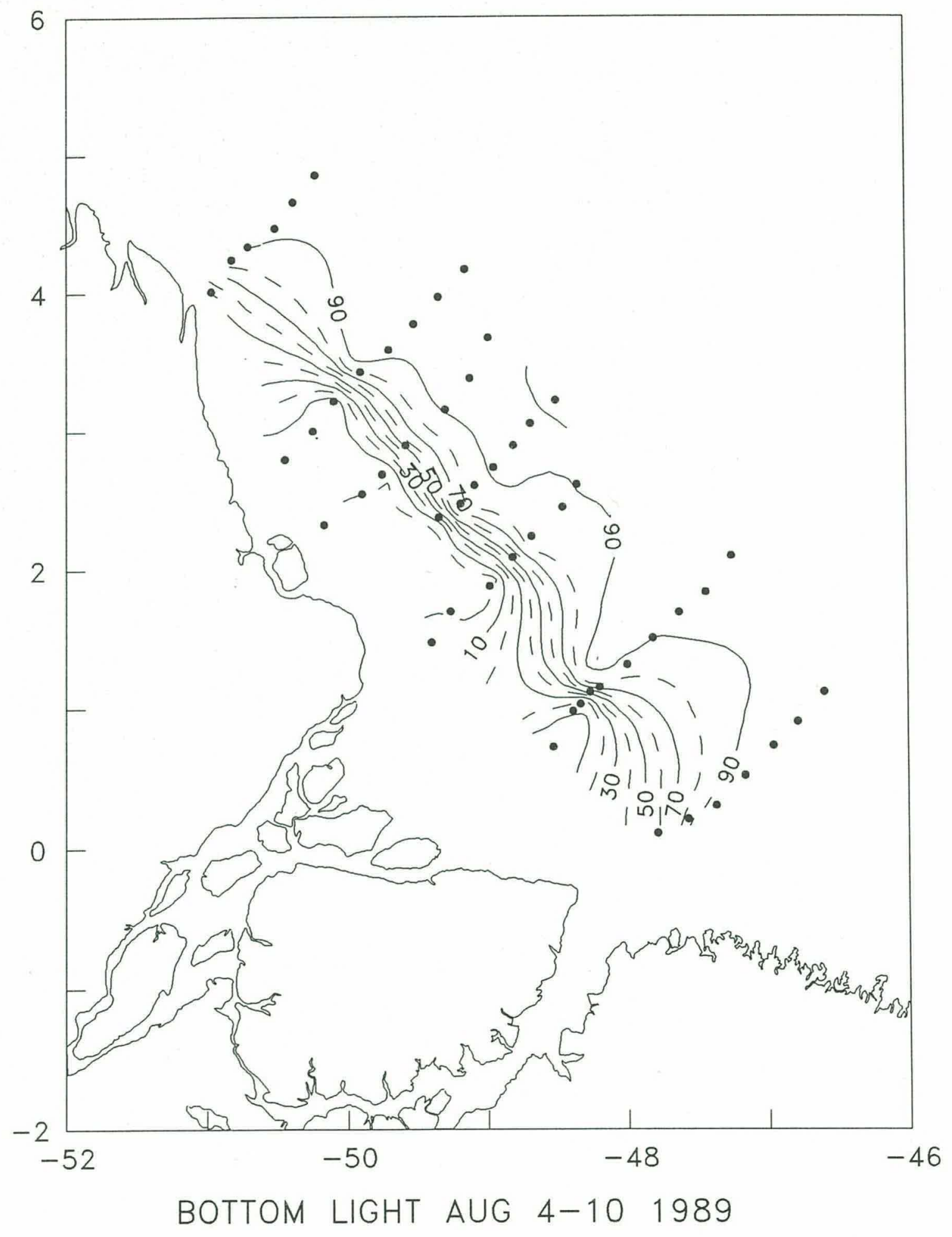



B. Time Series Anchor Station - August 11-12, 1989 Stations 54 to 79 


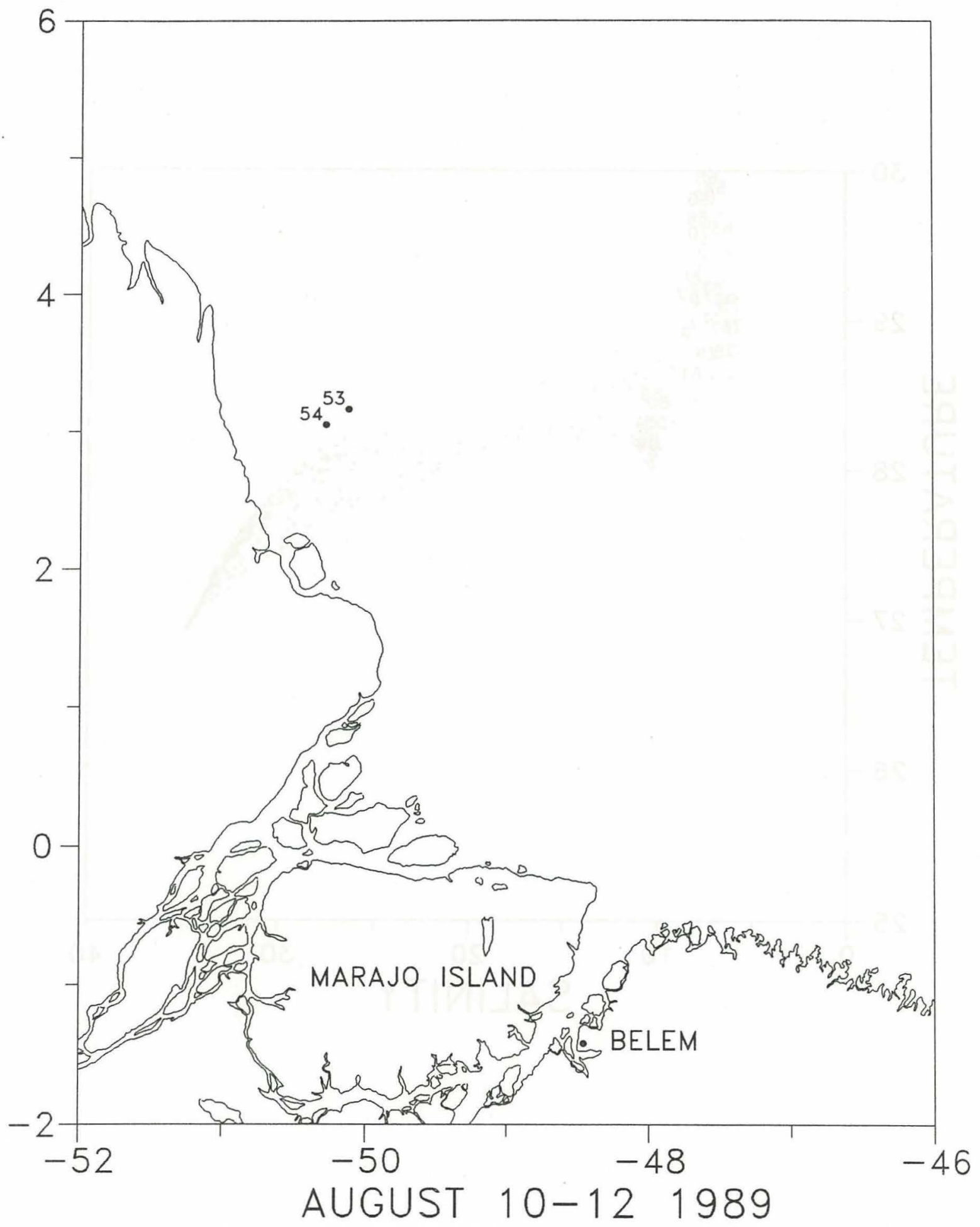




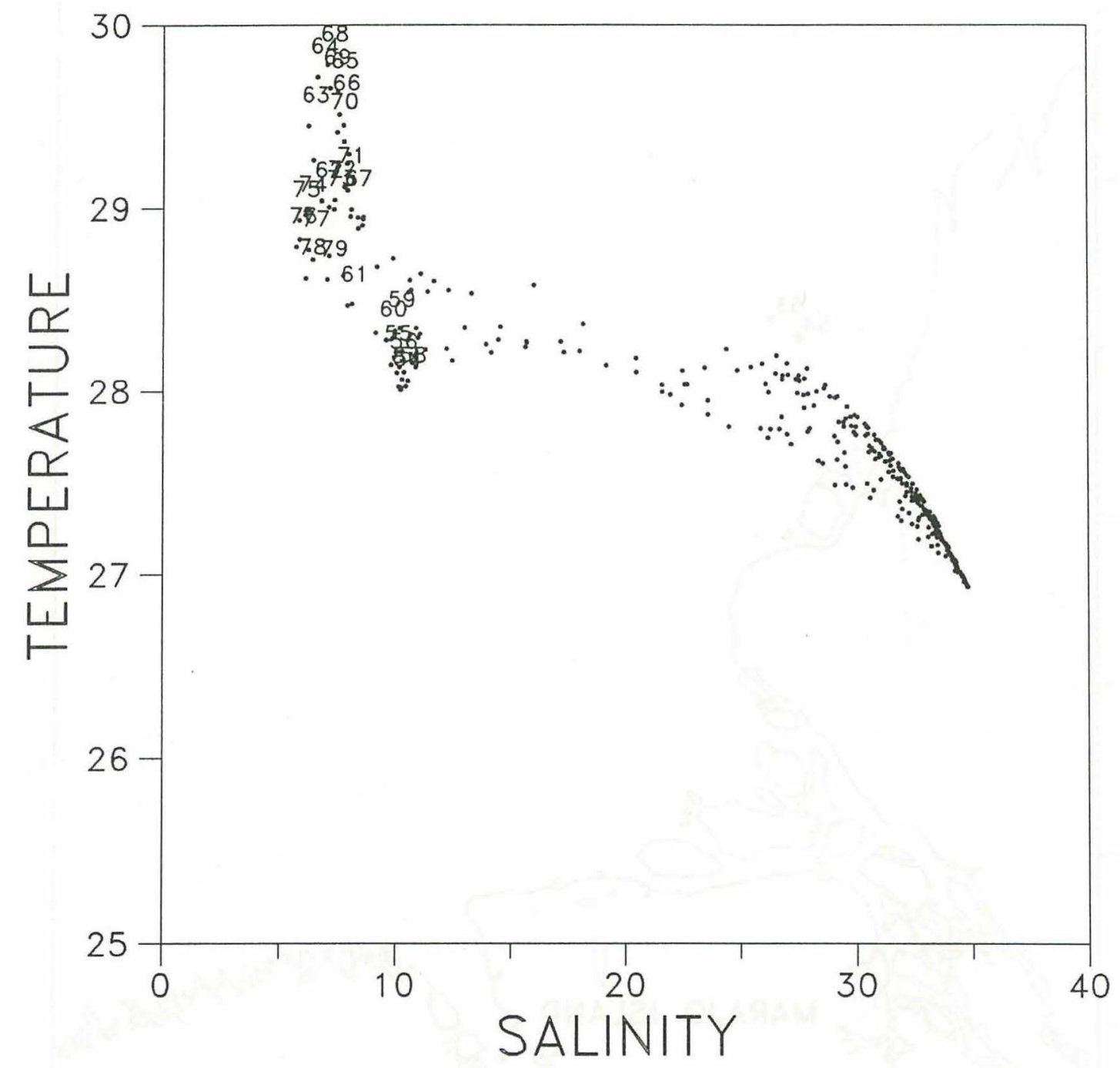


TEMPERATURE

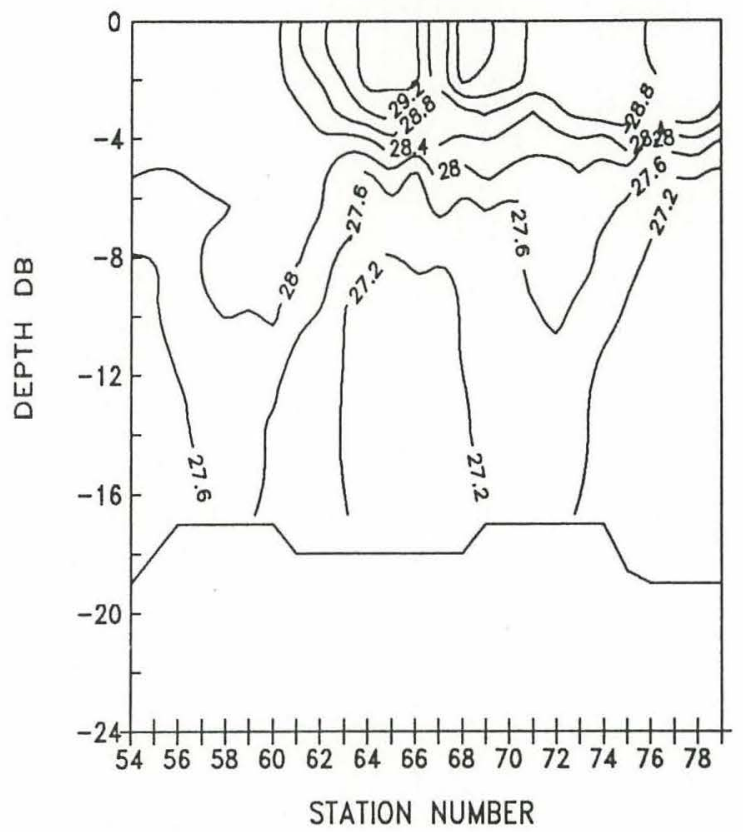

SIGMA-T

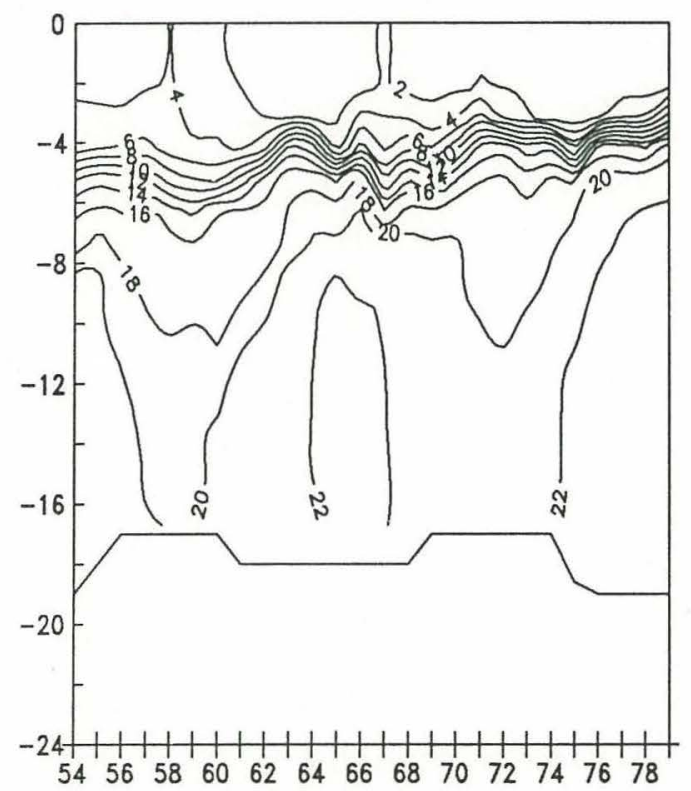

SALINITY

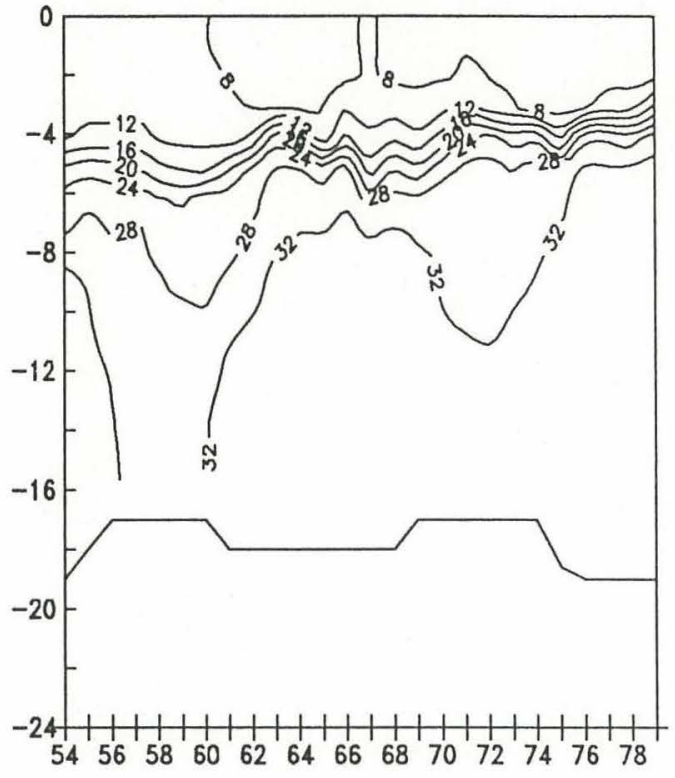

LIGHT PCT

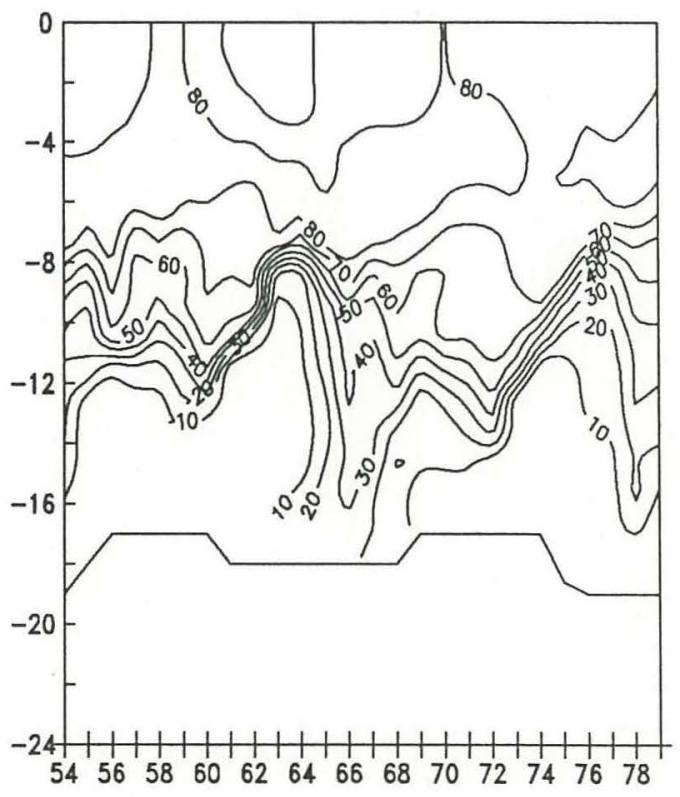



C. Section C - August $12-13,1989$ - Stations 80 to 86 


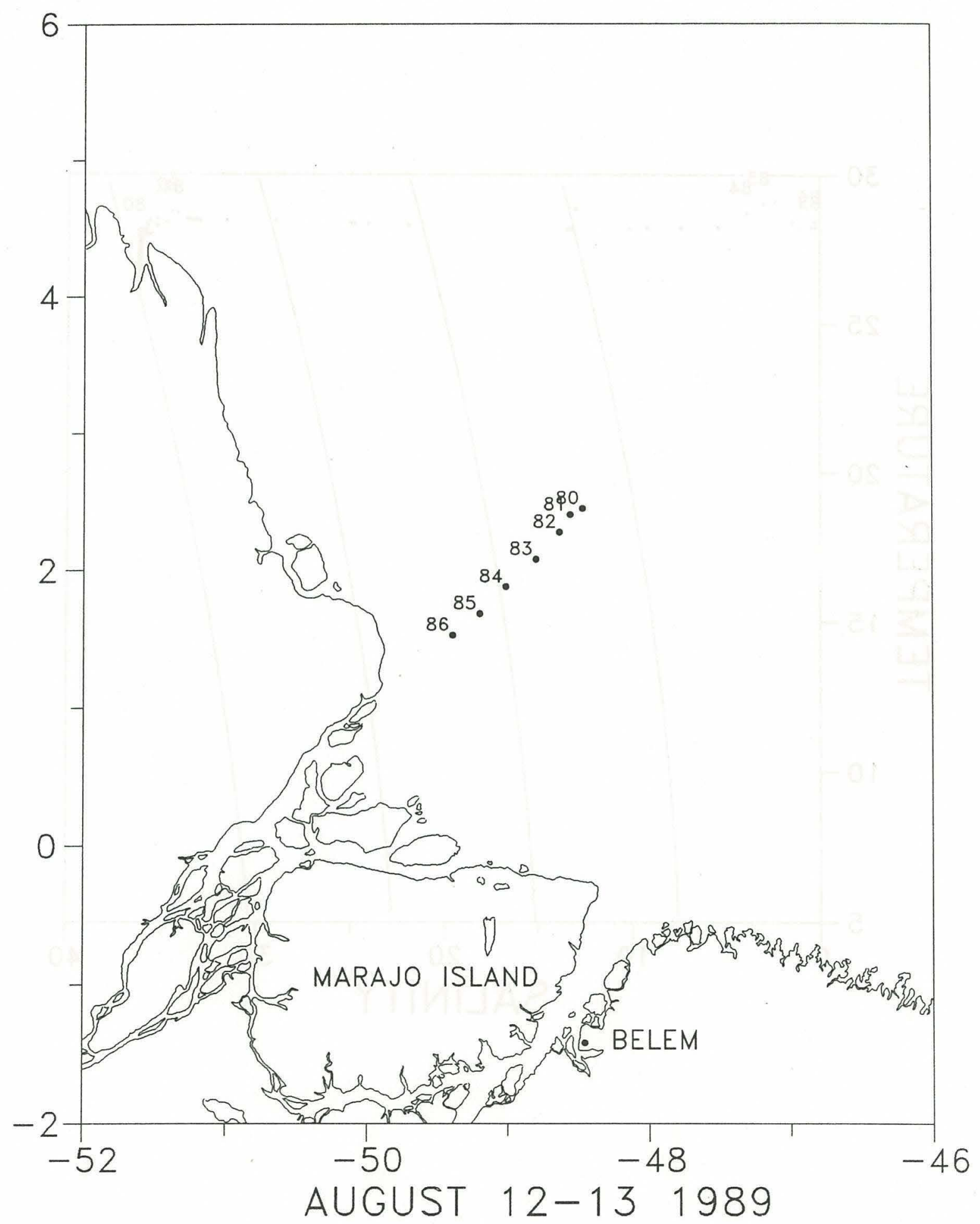




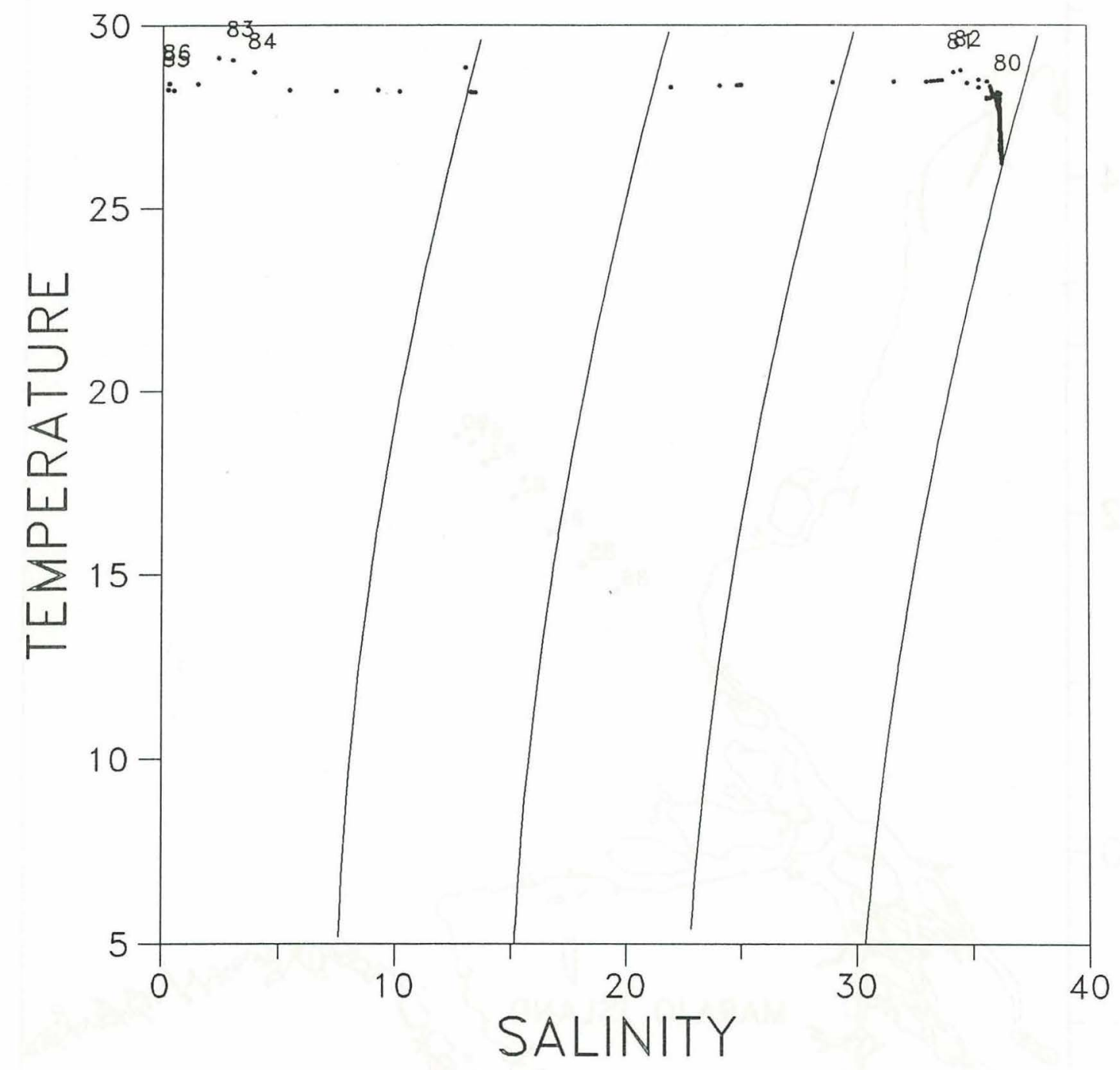



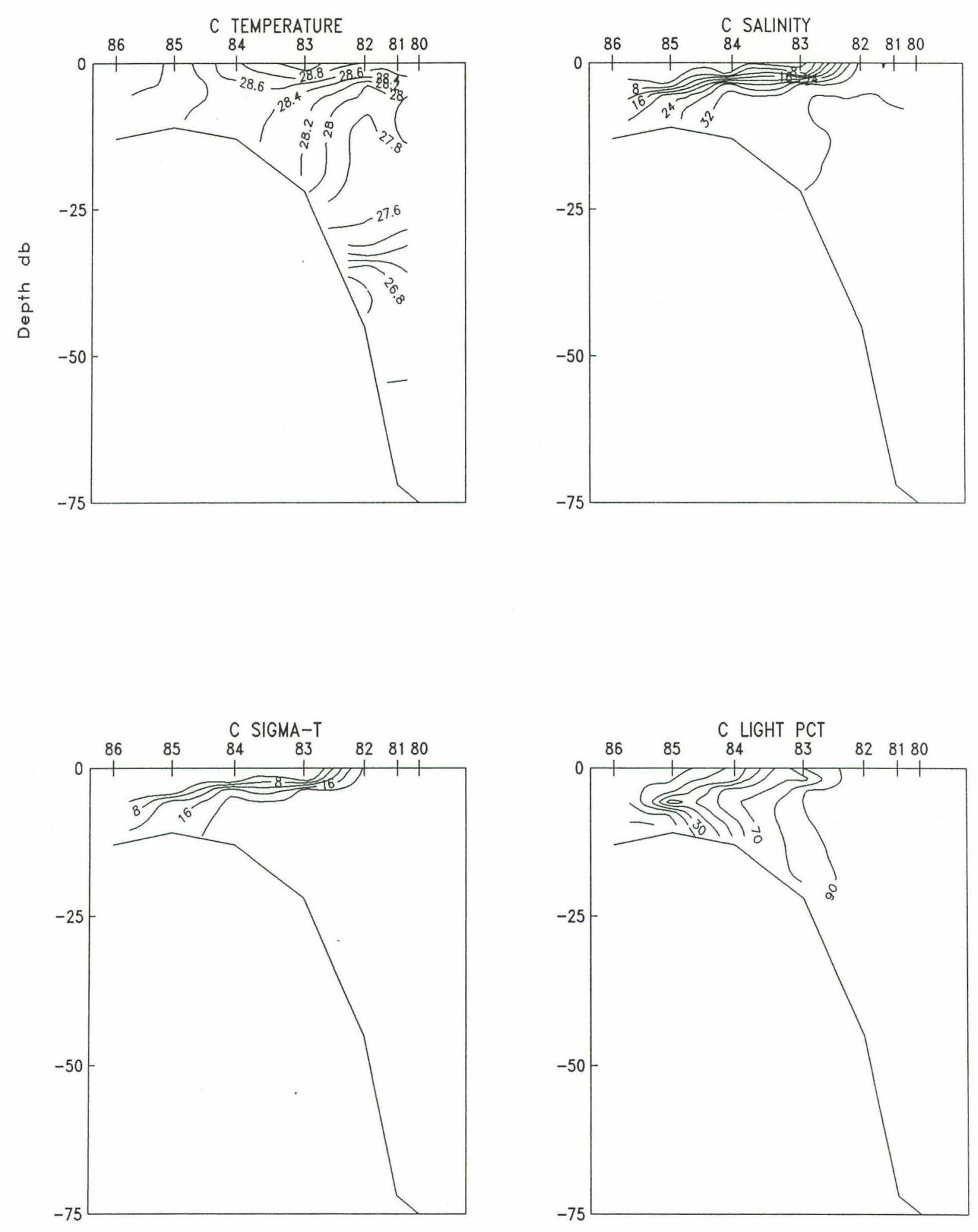
8. Station Listings and Profiles 
Cruise 18909 Consecutive Station \# 1 Depth m:27 Time: 1989-08-04 06:35 Position: $0^{\circ} 6.50^{\prime} \mathrm{N} \quad 47^{\circ} 47.20 \mathrm{~W}$ Depth Deck Offset: $0.90 \quad$ Fish 1

\begin{tabular}{|c|c|c|c|c|c|c|c|c|}
\hline $\begin{array}{l}\text { PRESSURE } \\
\text { (d-bars) }\end{array}$ & Interp & $\begin{array}{l}\text { TEMP } \\
(\operatorname{deg} \mathrm{C})\end{array}$ & $\begin{array}{l}\text { SALINITY } \\
(0 / 00)\end{array}$ & SI GMA-T & DELTA-D & $\begin{array}{l}\text { OXYGEN } \\
(\mathrm{ml} / \mathrm{l})\end{array}$ & $\begin{array}{l}\text { CHANNELA } \\
\text { (\%light) }\end{array}$ & $\begin{array}{l}\text { CHANNELB } \\
\text { (\%light) }\end{array}$ \\
\hline 0.0 & $E$ & 28.381 & 29.648 & 18.255 & 0.0000 & 0.000 & 0.0 & 83.8 \\
\hline 2.0 & & 28.381 & 29.647 & 18.255 & 0.0189 & 0.000 & 0.0 & 83.8 \\
\hline 4.0 & * & 28.381 & 29.648 & 18.255 & 0.0378 & 0.000 & 0.0 & 83.7 \\
\hline 6.0 & & 28.381 & 29.646 & 18.254 & 0.0566 & 0.000 & 0.0 & 83.8 \\
\hline 8.0 & & 28.470 & 31.105 & 19.318 & 0.0741 & 0.000 & 0.0 & 80.5 \\
\hline 10.0 & & 28.479 & 32.563 & 20.409 & 0.0896 & 0.000 & 0.0 & 81.0 \\
\hline 12.0 & & 28.467 & 32.921 & 20.682 & 0.1040 & 0.000 & 0.0 & 73.4 \\
\hline 14.0 & & 28.464 & 33.014 & 20.753 & 0.1182 & 0.000 & 0.0 & 69.2 \\
\hline 16.0 & * & 28.462 & 33.041 & 20.773 & 0.1323 & 0.000 & 0.0 & 64.2 \\
\hline 18.0 & & 28.462 & 33.068 & 20.794 & 0.1463 & 0.000 & 0.0 & 59.2 \\
\hline 20.0 & & 28.462 & 33.071 & 20.796 & 0.1603 & 0.000 & 0.0 & 56.3 \\
\hline 22.0 & & 28.462 & 33.077 & 20.801 & 0.1743 & 0.000 & 0.0 & 57.8 \\
\hline
\end{tabular}
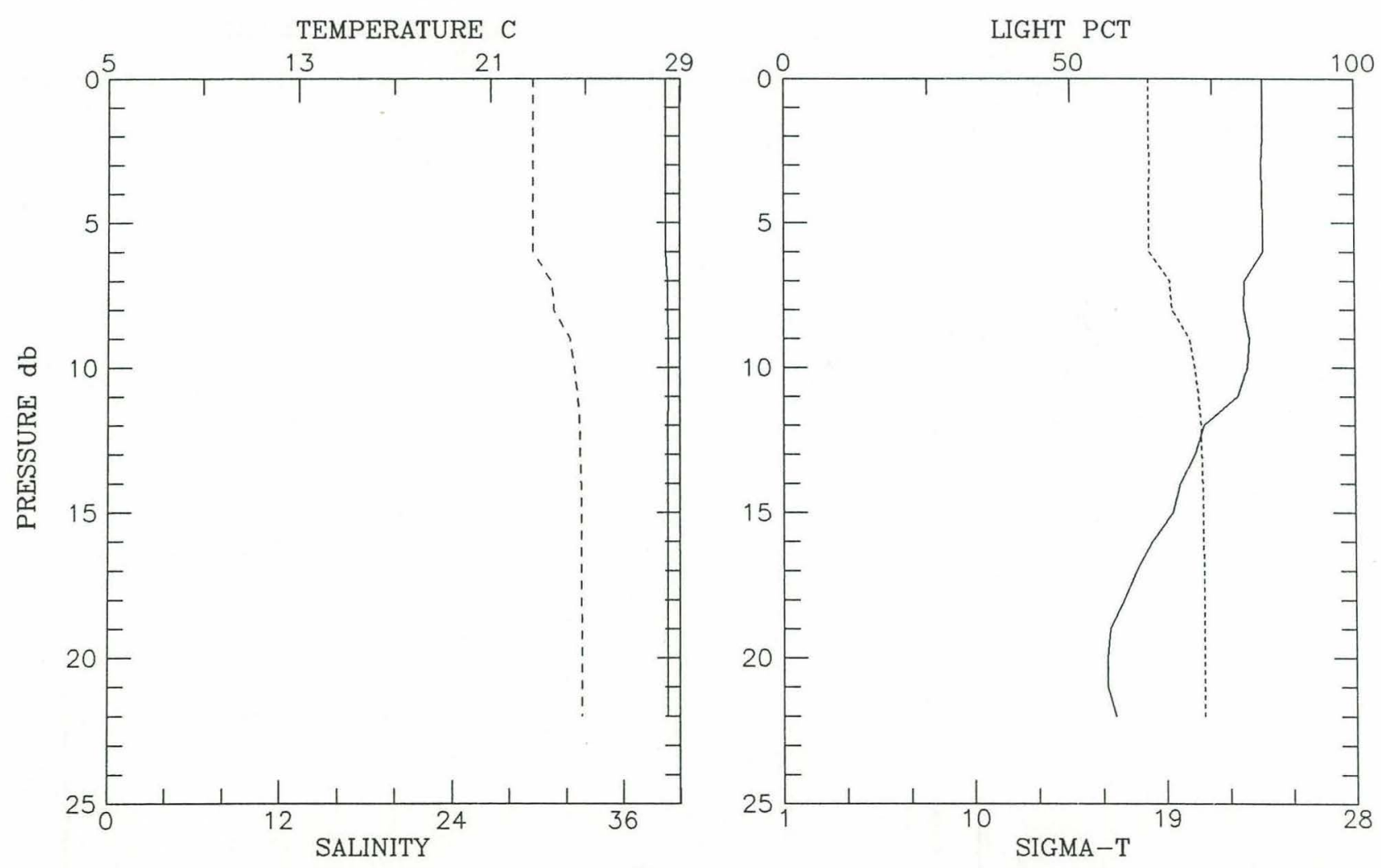


\begin{tabular}{|c|c|c|c|c|c|c|c|c|}
\hline $\begin{array}{l}\text { Cruise I8 } \\
\text { Position: }\end{array}$ & $\begin{array}{r}8909 \text { Cor } \\
0^{\circ} \quad 12\end{array}$ & 50 N & $47^{\circ} 34.10$ & 2 & epth Deck & offset: & 0.90 & Fish 1 \\
\hline $\begin{array}{l}\text { PRESSURE } \\
\text { (d-bars) }\end{array}$ & Interp & $\begin{array}{c}\text { TEMP } \\
(\operatorname{deg} \mathrm{C})\end{array}$ & $\begin{array}{c}\text { SALINITY } \\
(0 / 00)\end{array}$ & SIGMA-T & DELTA-D & $\begin{array}{l}\text { OXYGEN } \\
(m / / l)\end{array}$ & $\begin{array}{l}\text { CHANNELA } \\
\text { (\%light) }\end{array}$ & $\begin{array}{l}\text { CHANNELB } \\
\text { (\%light) }\end{array}$ \\
\hline 0.0 & $E$ & 28.442 & 34.507 & 21.881 & 0.0000 & 0.000 & 0.0 & 93.3 \\
\hline 2.0 & & 28.441 & 34.506 & 21.881 & 0.0119 & 0.000 & 0.0 & 93.3 \\
\hline 4.0 & & 28.442 & 34.505 & 21.880 & 0.0238 & 0.000 & 0.0 & 93.8 \\
\hline 6.0 & & 28.442 & 34.504 & 21.879 & 0.0357 & 0.000 & 0.0 & 95.3 \\
\hline 8.0 & & 28.444 & 34.503 & 21.878 & 0.0477 & 0.000 & 0.0 & 96.8 \\
\hline 10.0 & & 28.439 & 34.504 & 21.880 & 0.0596 & 0.000 & 0.0 & 93.3 \\
\hline 12.0 & & 28.437 & 34.504 & 21.880 & 0.0715 & 0.000 & 0.0 & 93.4 \\
\hline 14.0 & & 28.437 & 34.504 & 21.881 & 0.0834 & 0.000 & 0.0 & 93.3 \\
\hline 16.0 & & 28.437 & 34.503 & 21.880 & 0.0954 & 0.000 & 0.0 & 93.3 \\
\hline 18.0 & & 28.436 & 34.503 & 21.880 & 0.1073 & 0.000 & 0.0 & 93.3 \\
\hline 20.0 & & 28.436 & 34.503 & 21.880 & 0.1192 & 0.000 & 0.0 & 93.3 \\
\hline 22.0 & & 28.435 & 34.503 & 21.880 & 0.1312 & 0.000 & 0.0 & 93.3 \\
\hline 24.0 & & 28.435 & 34.504 & 21.881 & 0.1431 & 0.000 & 0.0 & 93.2 \\
\hline 26.0 & & 28.432 & 34.509 & 21.886 & 0.1550 & 0.000 & 0.0 & 93.5 \\
\hline
\end{tabular}
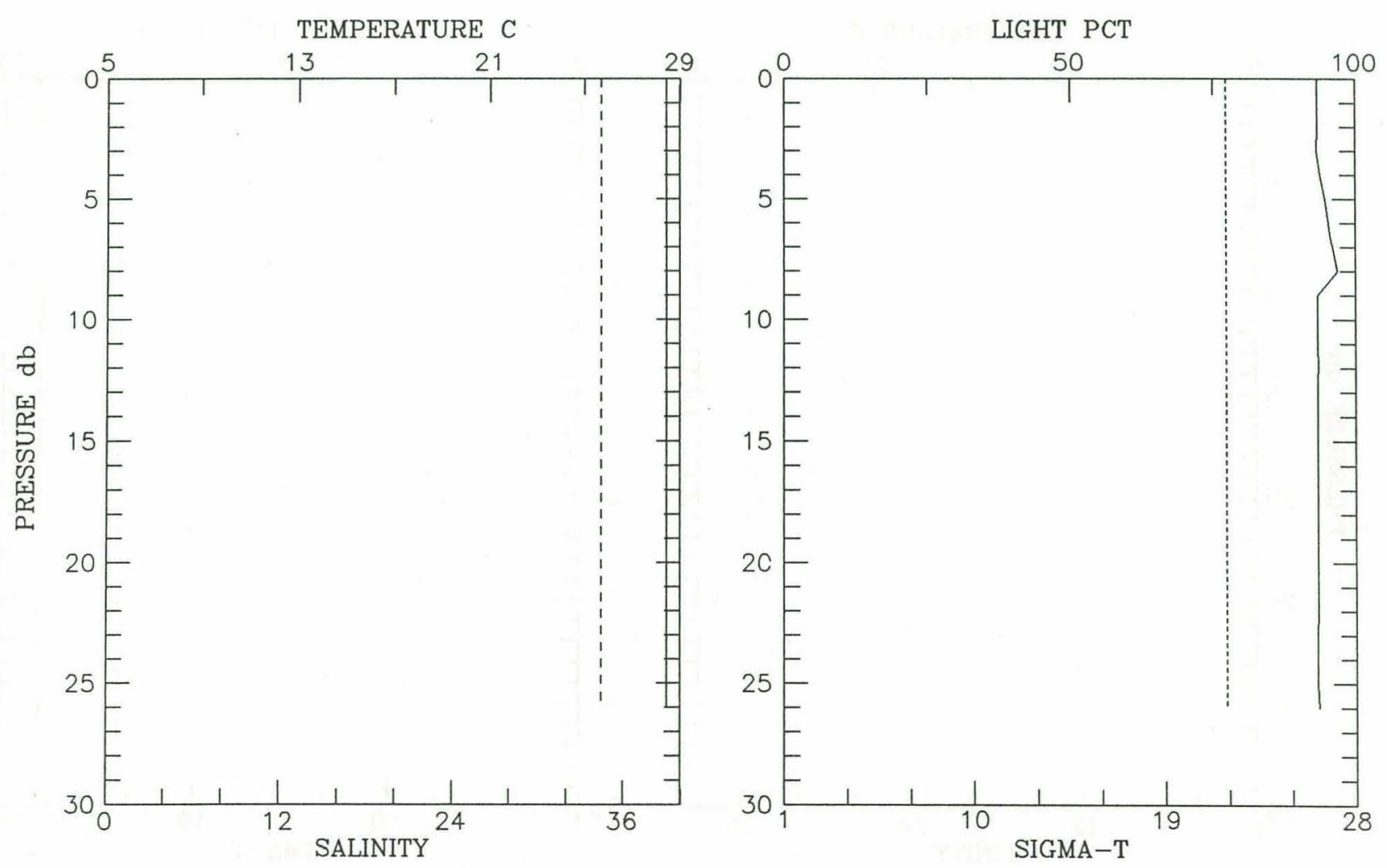


\begin{tabular}{|c|c|c|c|c|c|c|c|c|}
\hline \multirow{2}{*}{$\begin{array}{l}\text { Cruise I8 } \\
\text { Position: } \\
\text { PRESSURE } \\
\text { (d-bars) }\end{array}$} & \multicolumn{2}{|c|}{$=0^{\circ} 18.40^{\prime} \mathrm{N}$} & $47^{\circ} 22.00^{\prime} \mathrm{W}$ & \multicolumn{2}{|c|}{ Depth Deck } & Offset: & \multirow{2}{*}{$\begin{array}{r}0.70 \\
\text { CHANNELA } \\
\text { (\%light) }\end{array}$} & \multirow{2}{*}{$\begin{array}{r}\text { Fish } 1 \\
\text { CHANNELB } \\
(\% \text { light })\end{array}$} \\
\hline & Interp & $\begin{array}{c}\text { TEMP } \\
(\operatorname{deg} C)\end{array}$ & $\begin{array}{l}\text { SALINITY } \\
(0 / 00)\end{array}$ & SI GMA-T & DELTA-D & $\begin{array}{l}\text { OXYGEN } \\
(\mathrm{ml} / \mathrm{l})\end{array}$ & & \\
\hline 0.0 & E & 28.375 & 35.372 & 22.553 & 0.0000 & 0.000 & 0.0 & 93.8 \\
\hline 2.0 & & 28.375 & 35.371 & 22.553 & 0.0106 & 0.000 & 0.0 & 93.8 \\
\hline 4.0 & & 28.372 & 35.371 & 22.553 & 0.0213 & 0.000 & 0.0 & 93.7 \\
\hline 6.0 & & 28.371 & 35.371 & 22.554 & 0.0319 & 0.000 & 0.0 & 93.6 \\
\hline 8.0 & & 28.370 & 35.371 & 22.554 & 0.0425 & 0.000 & 0.0 & 93.5 \\
\hline 10.0 & & 28.369 & 35.372 & 22.555 & 0.0531 & 0.000 & 0.0 & 93.6 \\
\hline 12.0 & & 28.369 & 35.372 & 22.555 & 0.0638 & 0.000 & 0.0 & 93.6 \\
\hline 14.0 & & 28.368 & 35.373 & 22.556 & 0.0744 & 0.000 & 0.0 & 93.7 \\
\hline 16.0 & & 28.368 & 35.373 & 22.556 & 0.0850 & 0.000 & 0.0 & 93.5 \\
\hline 18.0 & & 28.369 & 35.374 & 22.557 & 0.0957 & 0.000 & 0.0 & 93.7 \\
\hline 20.0 & & 28.369 & 35.373 & 22.556 & 0.1063 & 0.000 & 0.0 & 93.6 \\
\hline 22.0 & & 28.368 & 35.374 & 22.557 & 0.1170 & 0.000 & 0.0 & 93.7 \\
\hline 24.0 & & 28.369 & 35.373 & 22.556 & 0.1276 & 0.000 & 0.0 & 93.6 \\
\hline 26.0 & & 28.368 & 35.375 & 22.558 & 0.1382 & 0.000 & 0.0 & 93.7 \\
\hline 28.0 & & 28.367 & 35.376 & 22.559 & 0.1489 & 0.000 & 0.0 & 93.6 \\
\hline 30.0 & & 28.368 & 35.376 & 22.559 & 0.1595 & 0.000 & 0.0 & 93.5 \\
\hline 32.0 & & 28.367 & 35.376 & 22.559 & 0.1702 & 0.000 & 0.0 & 93.7 \\
\hline
\end{tabular}
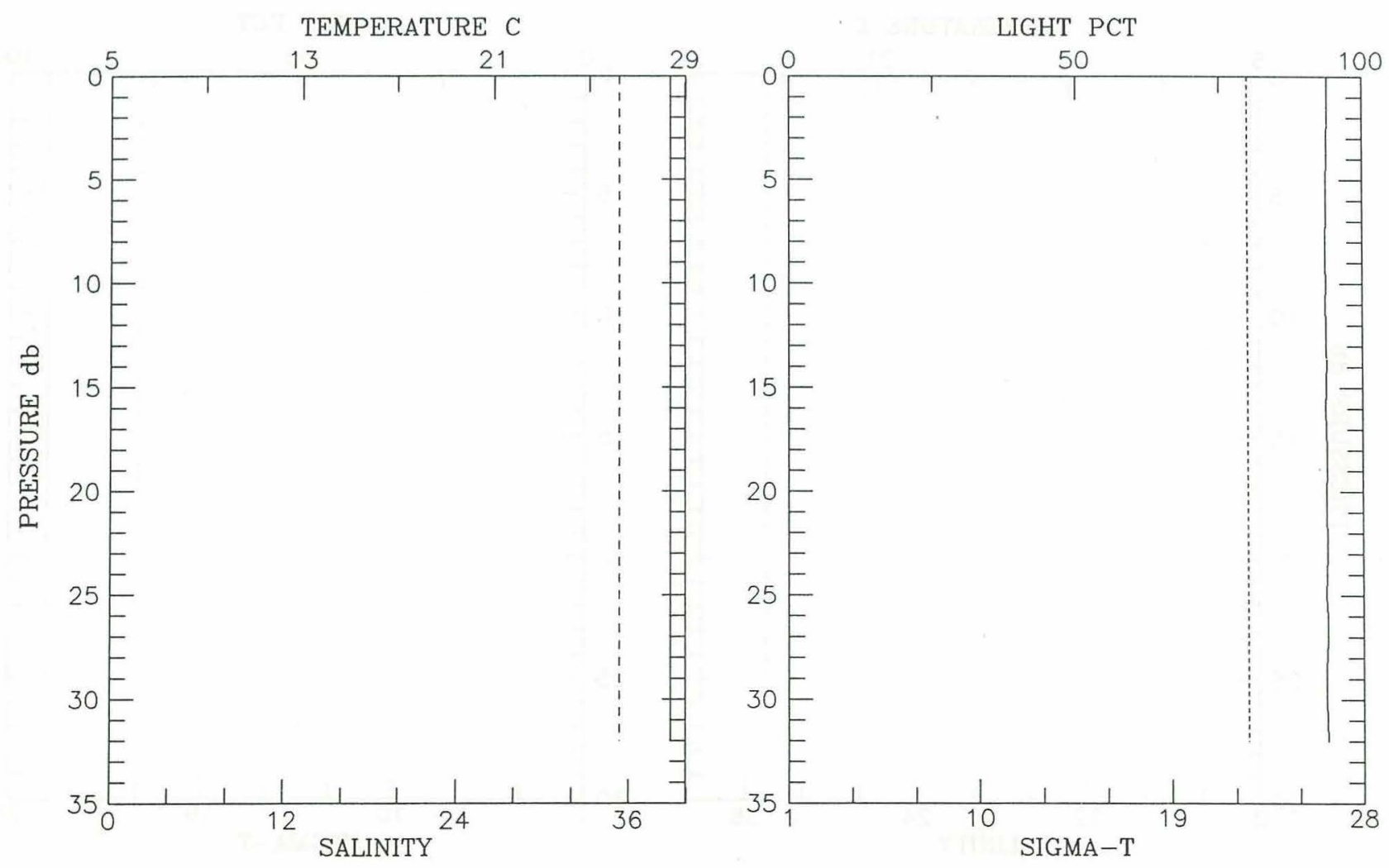


\begin{tabular}{|c|c|c|c|c|c|c|c|c|}
\hline \multirow{2}{*}{$\begin{array}{l}\text { Position: } \\
\text { PRESSURE } \\
\text { (d-bars) }\end{array}$} & \multicolumn{2}{|c|}{$=0^{\circ} 31.201 \mathrm{~N}$} & $47^{\circ} 9.70 \mathrm{~W}$ & \multicolumn{2}{|c|}{ Depth Deck } & Offset: & & \multirow{2}{*}{$\begin{array}{r}\text { Fish } 1 \\
\text { CHANNELB } \\
\text { (\%light) }\end{array}$} \\
\hline & Interp & $\begin{array}{l}\text { TEMP } \\
(\operatorname{deg} \mathrm{C})\end{array}$ & $\begin{array}{l}\text { SALINITY } \\
(0 / 00)\end{array}$ & SI GMA-T & DELTA-D & $\begin{array}{l}\text { OXYGEN } \\
(\mathrm{ml} / \mathrm{l})\end{array}$ & $\begin{array}{l}\text { CHANNELA } \\
\text { (\%light) }\end{array}$ & \\
\hline 0.0 & E & 28.181 & 35.792 & 22.933 & 0.0000 & 0.000 & 0.0 & 94.2 \\
\hline 2.0 & $E$ & 28.181 & 35.792 & 22.933 & 0.0099 & 0.000 & 0.0 & 94.2 \\
\hline 4.0 & & 28.185 & 35.792 & 22.932 & 0.0198 & 0.000 & 0.0 & 94.3 \\
\hline 6.0 & & 28.192 & 35.792 & 22.929 & 0.0297 & 0.000 & 0.0 & 94.5 \\
\hline 8.0 & & 28.164 & 35.792 & 22.939 & 0.0396 & 0.000 & 0.0 & 94.5 \\
\hline 10.0 & & 28.156 & 35.790 & 22.940 & 0.0495 & 0.000 & 0.0 & 94.4 \\
\hline 12.0 & & 28.074 & 35.801 & 22.975 & 0.0 & 0.000 & 0.0 & 94.4 \\
\hline 14.0 & & 28.058 & 35.805 & 22.983 & 0.0692 & 0.000 & 0.0 & 94.4 \\
\hline 16.0 & & 28.058 & 35.805 & 22.983 & 0.0790 & 0.000 & 0.0 & 94.3 \\
\hline 18.0 & & 28.059 & 35.805 & 22.983 & 0.0888 & 0.000 & 0.0 & 94.4 \\
\hline 20.0 & & 28.045 & 35.810 & 22.991 & 0.0986 & 0.000 & 0.0 & 94.4 \\
\hline 22.0 & & 28.045 & 35.811 & 22.992 & 0.1084 & 0.000 & 0.0 & 94.3 \\
\hline 24.0 & & 28.044 & 35.811 & 22.992 & 0.1182 & 0.000 & 0.0 & 94.3 \\
\hline 26.0 & & 28.033 & 35.817 & 23.001 & 0.1280 & 0.000 & 0.0 & 94.3 \\
\hline 28.0 & & 28.032 & 35.817 & 23.001 & 0.1378 & 0.000 & 0.0 & 94.3 \\
\hline 30.0 & & 28.023 & 35.822 & 23.008 & 0.1476 & 0.000 & 0.0 & 94.3 \\
\hline 32.0 & & 28.014 & 35.826 & 23.014 & 0.1574 & 0.000 & 0.0 & 94.3 \\
\hline 34.0 & & 28.007 & 35.830 & 23.019 & 0.1672 & 0.000 & 0.0 & 94.3 \\
\hline 36.0 & & 27.999 & 35.834 & 23.025 & 0.1769 & 0.000 & 0.0 & 94.3 \\
\hline 38.0 & & 27.994 & 35.837 & 23.028 & 0.1867 & 0.000 & 0.0 & 94.3 \\
\hline 40.0 & & 27.990 & 35.840 & 23.032 & 0.1964 & 0.000 & 0.0 & 94.1 \\
\hline
\end{tabular}
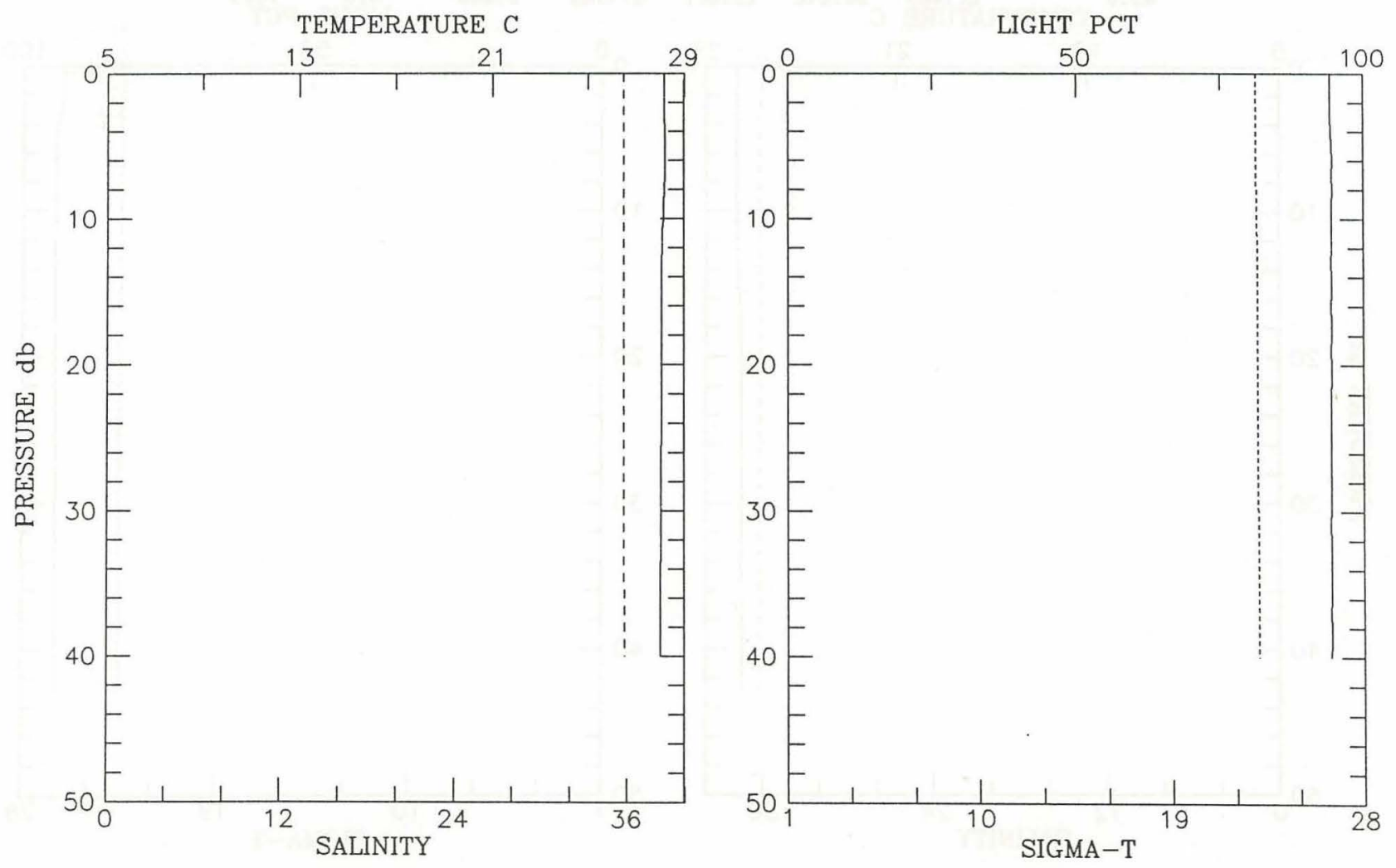


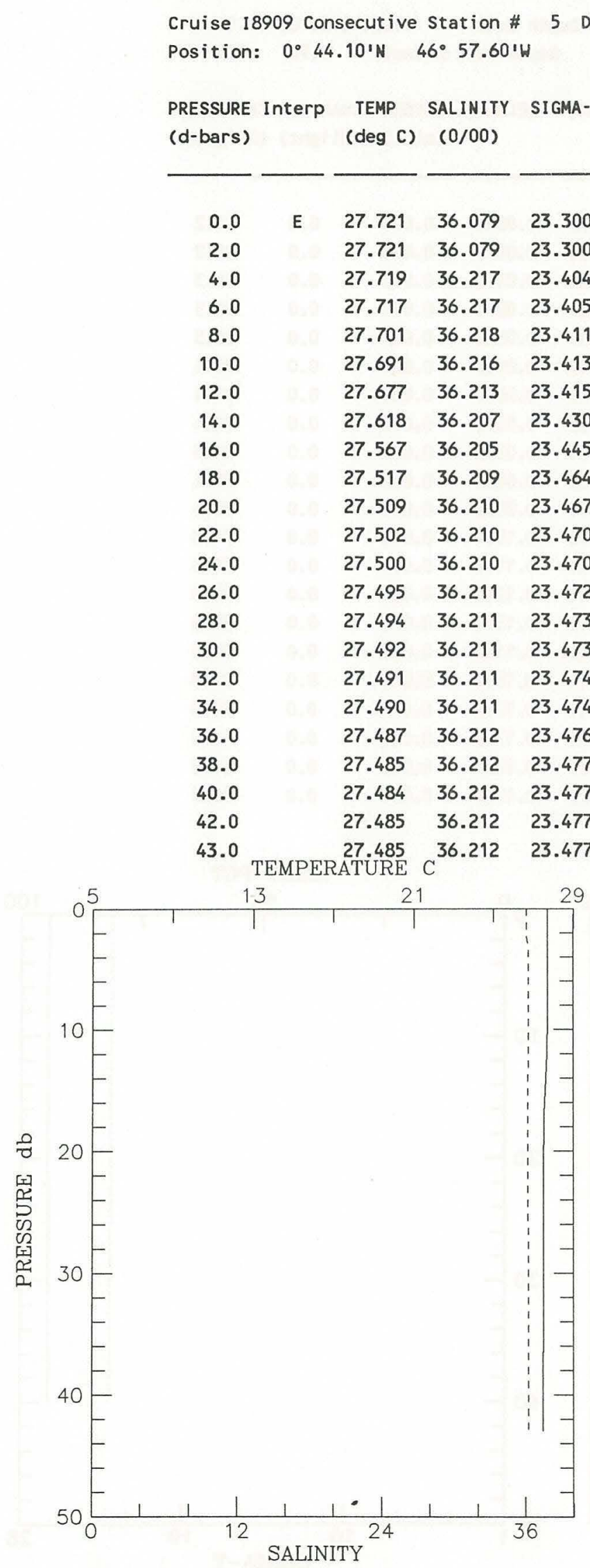

Time: 1989-08-04 17:06

Depth Deck offset:

$0.70 \quad$ Fish 1

PRESSURE InterP TEMP SALINITY SIGMA-T DELTA-D OXYGEN CHANNELA CHANNELB

(ml/l) (\%light) (\%light)

\begin{tabular}{|c|c|c|c|c|c|c|c|c|}
\hline 0.0 & $E$ & 27.721 & 36.079 & 23.300 & 0.0000 & 0.000 & 0.0 & 93.1 \\
\hline 2.0 & & 27.721 & 36.079 & 23.300 & 0.0092 & 0.000 & 0.0 & 93.1 \\
\hline 4.0 & & 27.719 & 36.217 & 23.404 & 0.0183 & 0.000 & 0.0 & 93.9 \\
\hline 6.0 & & 27.717 & 36.217 & 23.405 & 0.0273 & 0.000 & 0.0 & 94.3 \\
\hline 8.0 & & 27.701 & 36.218 & 23.411 & 0.0363 & 0.000 & 0.0 & 94.4 \\
\hline 10.0 & & 27.691 & 36.216 & 23.413 & 0.0453 & 0.000 & 0.0 & 94.4 \\
\hline 12.0 & & 27.677 & 36.213 & 23.415 & 0.0542 & 0.000 & 0.0 & 94.5 \\
\hline 14.0 & & 27.618 & 36.207 & 23.430 & 0.0632 & 0.000 & 0.0 & 94.4 \\
\hline 16.0 & & 27.567 & 36.205 & 23.445 & 0.0722 & 0.000 & 0.0 & 94.4 \\
\hline 18.0 & & 27.517 & 36.209 & 23.464 & 0.0811 & 0.000 & 0.0 & 94.4 \\
\hline 20.0 & & 27.509 & 36.210 & 23.467 & 0.0900 & 0.000 & 0.0 & 94.4 \\
\hline 22.0 & & 27.502 & 36.210 & 23.470 & 0.0989 & 0.000 & 0.0 & 94.3 \\
\hline 24.0 & & 27.500 & 36.210 & 23.470 & 0.1078 & 0.000 & 0.0 & 94.3 \\
\hline 26.0 & & 27.495 & 36.211 & 23.472 & 0.1167 & 0.000 & 0.0 & 94.3 \\
\hline 28.0 & & 27.494 & 36.211 & 23.473 & 0.1256 & 0.000 & 0.0 & 94.3 \\
\hline 30.0 & & 27.492 & 36.211 & 23.473 & 0.1345 & 0.000 & 0.0 & 94.3 \\
\hline 32.0 & & 27.491 & 36.211 & 23.474 & 0.1434 & 0.000 & 0.0 & 94.3 \\
\hline 34.0 & & 27.490 & 36.211 & 23.474 & 0.1523 & 0.000 & 0.0 & 94.3 \\
\hline 36.0 & & 27.487 & 36.212 & 23.476 & 0.1611 & 0.000 & 0.0 & 94.3 \\
\hline 38.0 & & 27.485 & 36.212 & 23.477 & 0.1700 & 0.000 & 0.0 & 94.3 \\
\hline 40.0 & & 27.484 & 36.212 & 23.477 & 0.1789 & 0.000 & 0.0 & 94.3 \\
\hline 42.0 & & 27.485 & 36.212 & 23.477 & 0.1878 & 0.000 & 0.0 & 94.3 \\
\hline 43.0 & & $\begin{array}{r}27.485 \\
\text { RATURE }\end{array}$ & 36.212 & 23.477 & 0.1923 & 0.000 & $\begin{array}{l}0.0 \\
\text { LIGHT }\end{array}$ & $\begin{array}{l}94.3 \\
\text { РCT }\end{array}$ \\
\hline
\end{tabular}

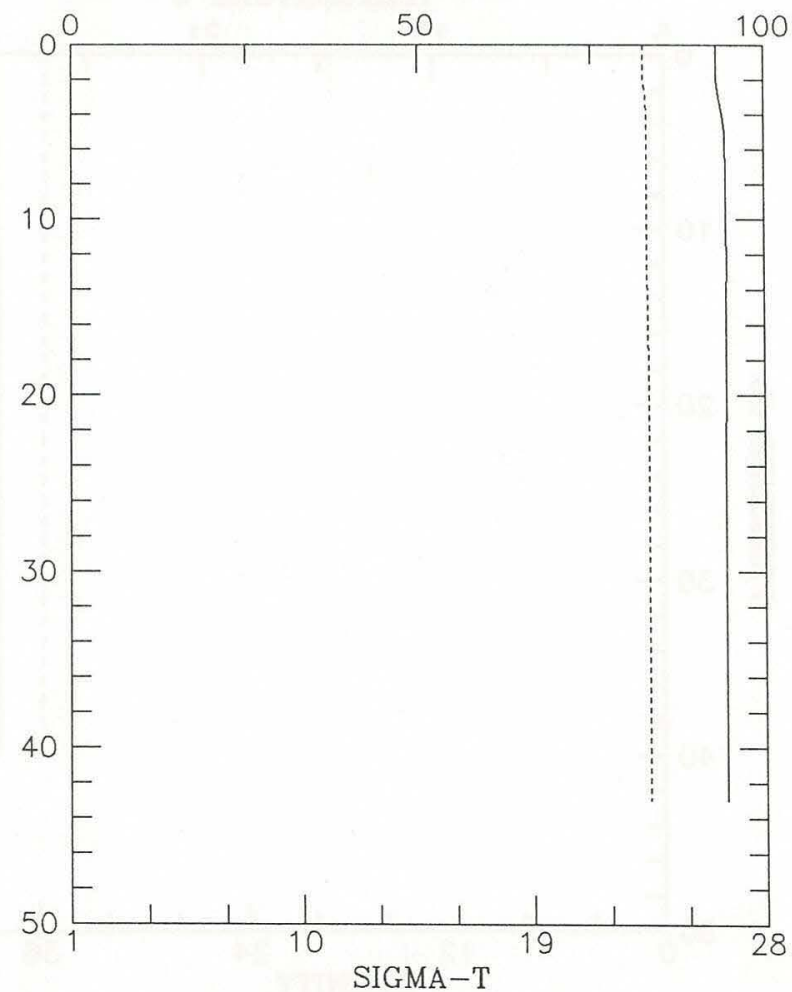




\begin{tabular}{|c|c|c|c|c|c|c|c|c|}
\hline $\begin{array}{l}\text { Cruise } 18 \\
\text { Position: }\end{array}$ & $\begin{array}{l}3909 \text { Con } \\
=0^{\circ} 54\end{array}$ & .30 N & $46^{\circ} 47.20^{\prime}$ & $W$ & epth Deck & offset: & -0.20 & Fish 1 \\
\hline $\begin{array}{l}\text { PRESSURE } \\
\text { (d-bars) }\end{array}$ & Interp & $\begin{array}{c}\text { TEMP } \\
(\operatorname{deg} C)\end{array}$ & $\begin{array}{l}\text { SALINITY } \\
(0 / 00)\end{array}$ & SIGMA-T & DELTA-D & $\begin{array}{l}\text { OXYGEN } \\
(\mathrm{ml} / \mathrm{l})\end{array}$ & $\begin{array}{l}\text { CHANNELA } \\
\text { (\%light) }\end{array}$ & $\begin{array}{l}\text { CHANNELB } \\
\text { (\%light) }\end{array}$ \\
\hline 0.0 & E & 27.383 & 36.240 & 23.531 & 0.0000 & 0.000 & 0.0 & 93.6 \\
\hline 5.0 & & 27.356 & 36.239 & 23.539 & 0.0219 & 0.000 & 0.0 & 94.6 \\
\hline 10.0 & & 27.314 & 36.236 & 23.550 & 0.0437 & 0.000 & 0.0 & 94.6 \\
\hline 15.0 & & 27.282 & 36.234 & 23.559 & 0.0655 & 0.000 & 0.0 & 94.5 \\
\hline 20.0 & & 27.230 & 36.233 & 23.575 & 0.0873 & 0.000 & 0.0 & 94.5 \\
\hline 25.0 & & 27.199 & 36.233 & 23.585 & 0.1090 & 0.000 & 0.0 & 94.4 \\
\hline 30.0 & & 27.187 & 36.233 & 23.589 & 0.1307 & 0.000 & 0.0 & 94.5 \\
\hline 35.0 & & 27.180 & 36.233 & 23.591 & 0.1524 & 0.000 & 0.0 & 94.5 \\
\hline 40.0 & & 27.180 & 36.233 & 23.591 & 0.1741 & 0.000 & 0.0 & 94.5 \\
\hline 45.0 & & 27.180 & 36.233 & 23.591 & 0.1958 & 0.000 & 0.0 & 94.6 \\
\hline 47.0 & & 27.180 & 36.233 & 23.591 & 0.2045 & 0.000 & 0.0 & 94.5 \\
\hline
\end{tabular}
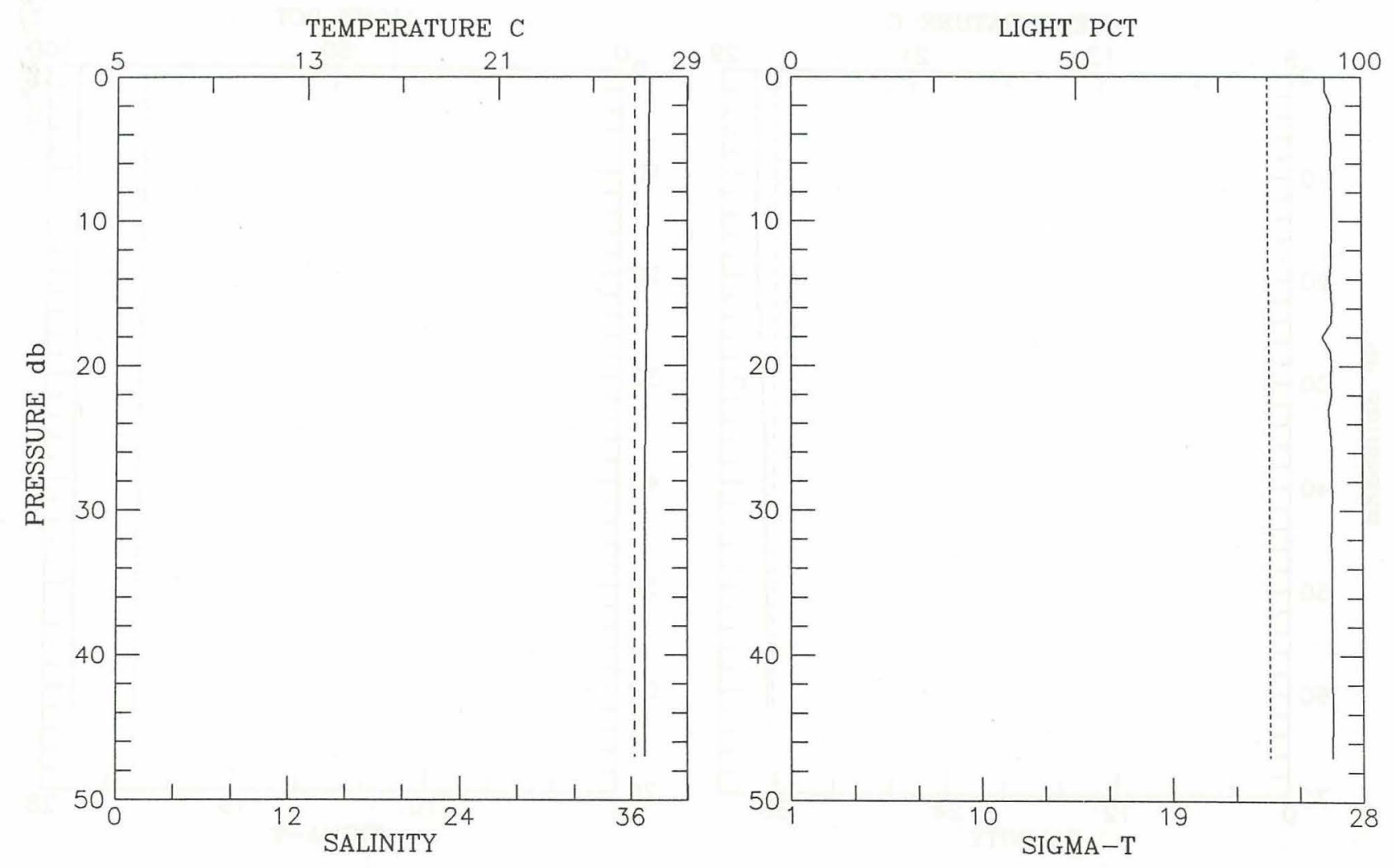


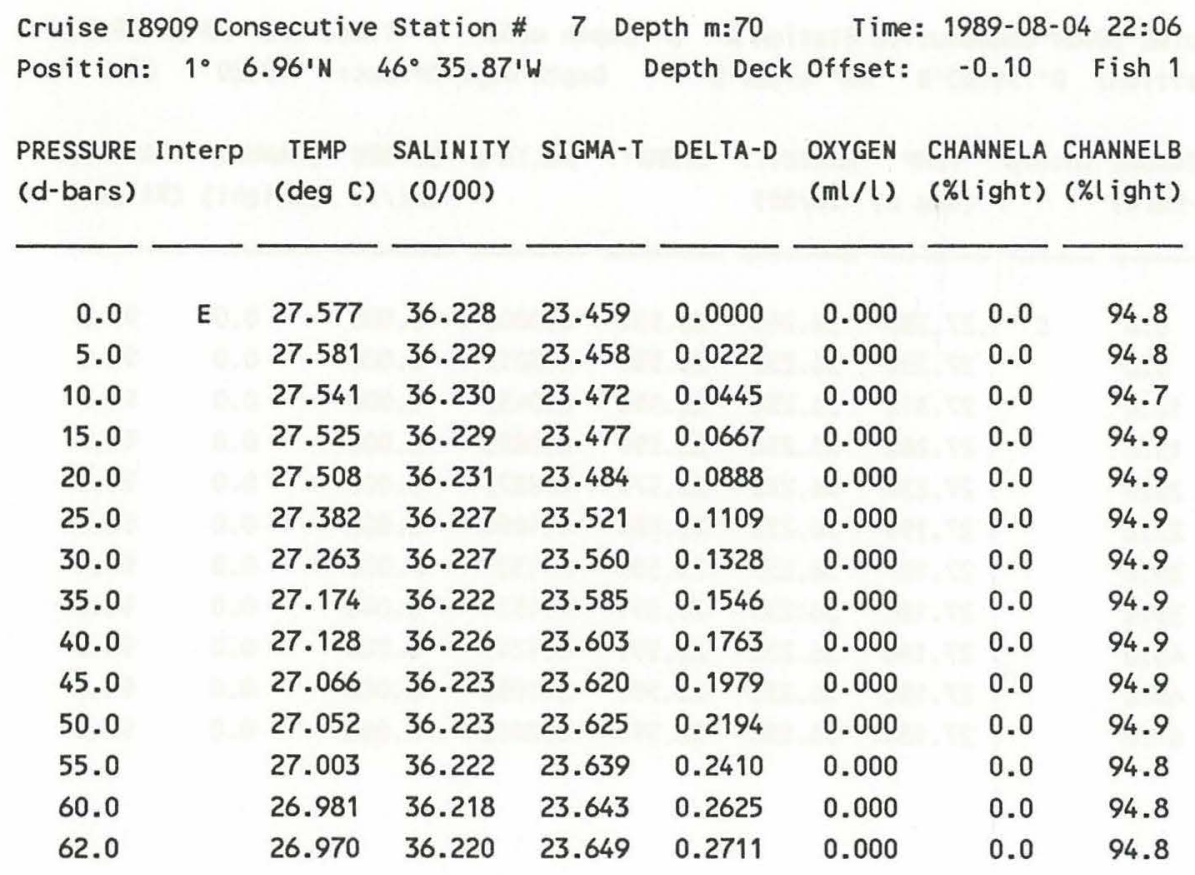
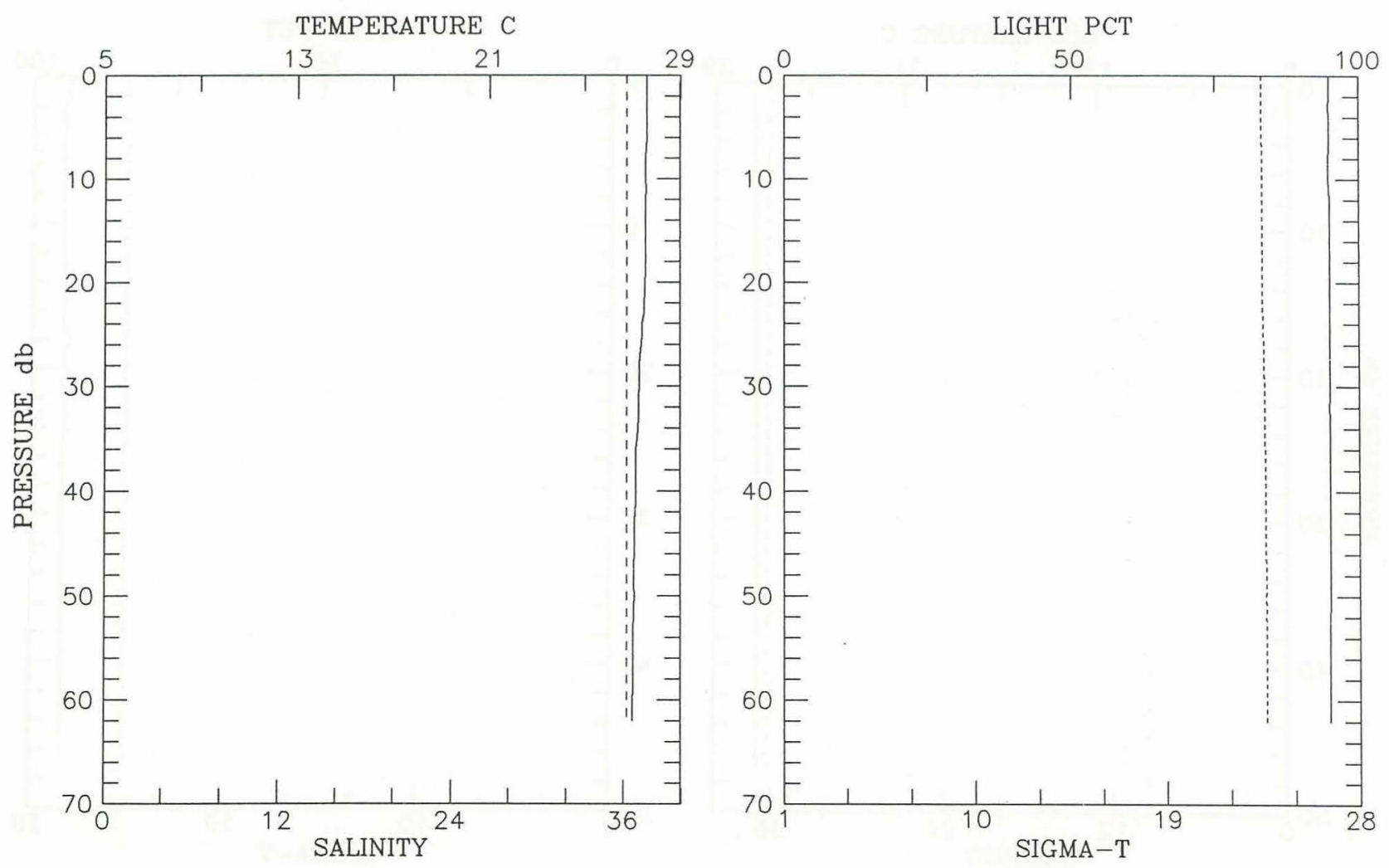


\begin{tabular}{|c|c|c|c|c|c|c|c|c|}
\hline \multicolumn{4}{|c|}{ Cruise 18909 Consecutive Station \# } & \multicolumn{2}{|c|}{8 Depth m: 140} & Tine & \multicolumn{2}{|c|}{ : $1989-08-05 \quad 04: 49$} \\
\hline Position: & $2^{\circ}$ & $.001 \mathrm{~N}$ & $47^{\circ} 15.10^{\prime}$ & & epth Deck & Offset: & -0.10 & Fish 1 \\
\hline $\begin{array}{l}\text { PRESSURE } \\
\text { (d-bars) }\end{array}$ & Interp & $\begin{array}{l}\text { TEMP } \\
(\operatorname{deg} \mathrm{C})\end{array}$ & $\begin{array}{l}\text { SALINITY } \\
(0 / 00)\end{array}$ & SIGMA-T & DELTA-D & $\begin{array}{l}\text { OXYGEN } \\
(\mathrm{ml} / \mathrm{l})\end{array}$ & $\begin{array}{l}\text { CHANNELA } \\
\text { (\%light) }\end{array}$ & $\begin{array}{l}\text { CHANNELB } \\
\text { (\%tight) }\end{array}$ \\
\hline 0.0 & E & 27.379 & 36.196 & 23.499 & 0.0000 & 0.000 & 0.0 & 93.7 \\
\hline 10.0 & & 27.383 & 36.203 & 23.503 & 0.0441 & 0.000 & 0.0 & 95.2 \\
\hline 20.0 & & 27.369 & 36.206 & 23.510 & 0.0882 & 0.000 & 0.0 & 95.1 \\
\hline 30.0 & & 27.307 & 36.216 & 23.537 & 0.1322 & 0.000 & 0.0 & 95.1 \\
\hline 40.0 & & 27.120 & 36.232 & 23.609 & 0.1758 & 0.000 & 0.0 & 95.1 \\
\hline 50.0 & & 26.875 & 36.258 & 23.708 & 0.2185 & 0.000 & 0.0 & 95.0 \\
\hline 60.0 & & 26.844 & 36.279 & 23.733 & 0.2607 & 0.000 & 0.0 & 95.1 \\
\hline 70.0 & & 26.800 & 36.310 & 23.771 & 0.3027 & 0.000 & 0.0 & 95.1 \\
\hline 80.0 & & 26.709 & 36.326 & 23.812 & 0.3445 & 0.000 & 0.0 & 95.1 \\
\hline 90.0 & & 26.413 & 36.353 & 23.926 & 0.3856 & 0.000 & 0.0 & 95.0 \\
\hline 100.0 & & 25.849 & 36.335 & 24.090 & 0.4252 & 0.000 & 0.0 & 94.8 \\
\hline 110.0 & & 24.997 & 36.272 & 24.306 & 0.4631 & 0.000 & 0.0 & 95.1 \\
\hline 120.0 & & 20.959 & 35.909 & 25.198 & 0.4977 & 0.000 & 0.0 & 94.7 \\
\hline 128.0 & & 17.605 & 35.913 & 26.070 & 0.5147 & 0.000 & 0.0 & 95.3 \\
\hline
\end{tabular}
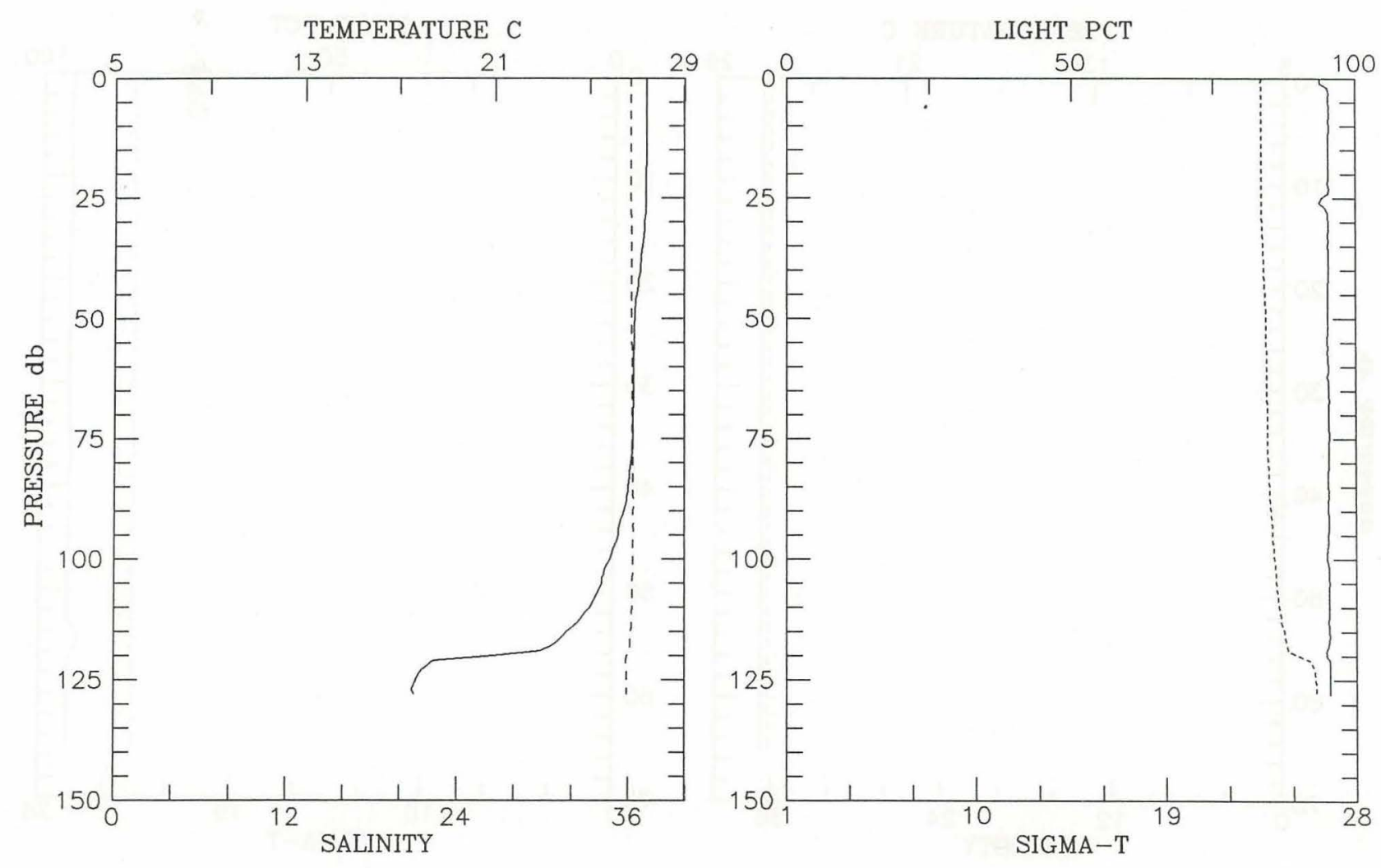


\begin{tabular}{|c|c|c|c|c|c|c|c|c|}
\hline \multicolumn{4}{|c|}{ Cruise 18909 Consecutive Station \# } & & Depth m:71 & $\begin{array}{r}\text { Time } \\
\text { offset: }\end{array}$ & \multicolumn{2}{|c|}{$1989-08-05 \quad 09: 33$} \\
\hline $\begin{array}{l}\text { PRESSURE } \\
\text { (d-bars) }\end{array}$ & Interp & $\begin{array}{c}\text { TEMP } \\
(\operatorname{deg} C)\end{array}$ & $\begin{array}{l}\text { SALINITY } \\
(0 / 00)\end{array}$ & SIGMA-T & DELTA-D & $\begin{array}{l}\text { OXYGEN } \\
(\mathrm{ml} / \mathrm{l})\end{array}$ & $\begin{array}{l}\text { CHANNELA } \\
(\% \text { light) }\end{array}$ & $\begin{array}{l}\text { CHANNELB } \\
\text { (\%light) }\end{array}$ \\
\hline 0.0 & E & 27.044 & 36.226 & 23.629 & 0.0000 & 0.000 & 0.0 & 94.6 \\
\hline 5.0 & & 27.054 & 36.226 & 23.626 & 0.0214 & 0.000 & 0.0 & 95.0 \\
\hline 10.0 & & 27.051 & 36.226 & 23.627 & 0.0429 & 0.000 & 0.0 & 95.0 \\
\hline 15.0 & & 27.054 & 36.226 & 23.626 & 0.0643 & 0.000 & 0.0 & 95.0 \\
\hline 20.0 & & 27.053 & 36.226 & 23.626 & 0.0858 & 0.000 & 0.0 & 95.1 \\
\hline 25.0 & & 27.054 & 36.226 & 23.626 & 0.1073 & 0.000 & 0.0 & 95.1 \\
\hline 30.0 & & 27.054 & 36.226 & 23.626 & 0.1288 & 0.000 & 0.0 & 95.1 \\
\hline 35.0 & & 27.053 & 36.226 & 23.626 & 0.1503 & 0.000 & 0.0 & 94.9 \\
\hline 40.0 & & 27.054 & 36.226 & 23.626 & 0.1719 & 0.000 & 0.0 & 95.0 \\
\hline 45.0 & & 27.054 & 36.226 & 23.626 & 0.1934 & 0.000 & 0.0 & 94.9 \\
\hline 50.0 & & 27.054 & 36.226 & 23.626 & 0.2149 & 0.000 & 0.0 & 95.1 \\
\hline 55.0 & & 27.054 & 36.226 & 23.626 & 0.2365 & 0.000 & 0.0 & 93.3 \\
\hline 60.0 & & 27.055 & 36.226 & 23.626 & 0.2581 & 0.000 & 0.0 & 95.0 \\
\hline 65.0 & & 27.055 & 36.226 & 23.626 & 0.2797 & 0.000 & 0.0 & 95.0 \\
\hline
\end{tabular}
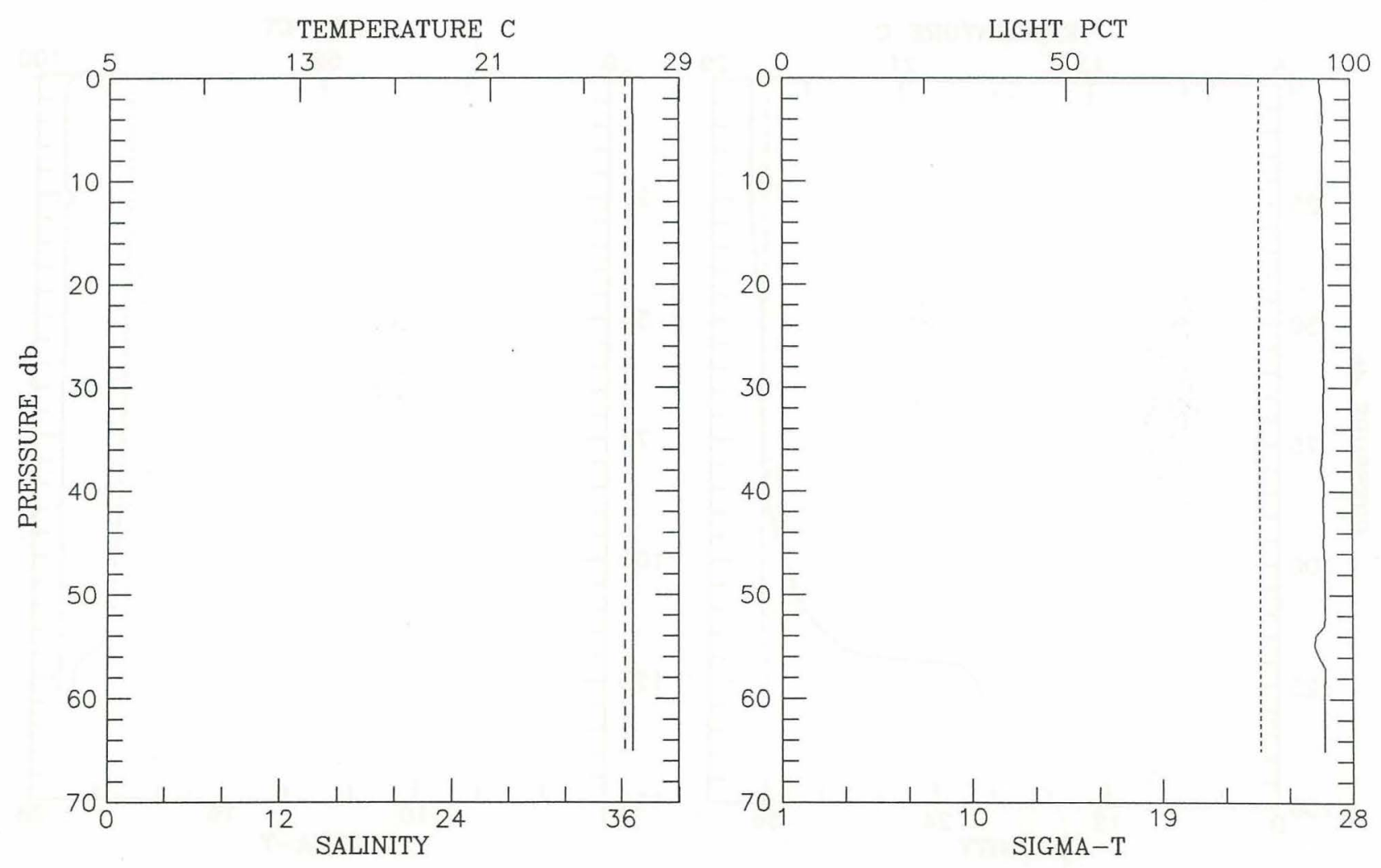


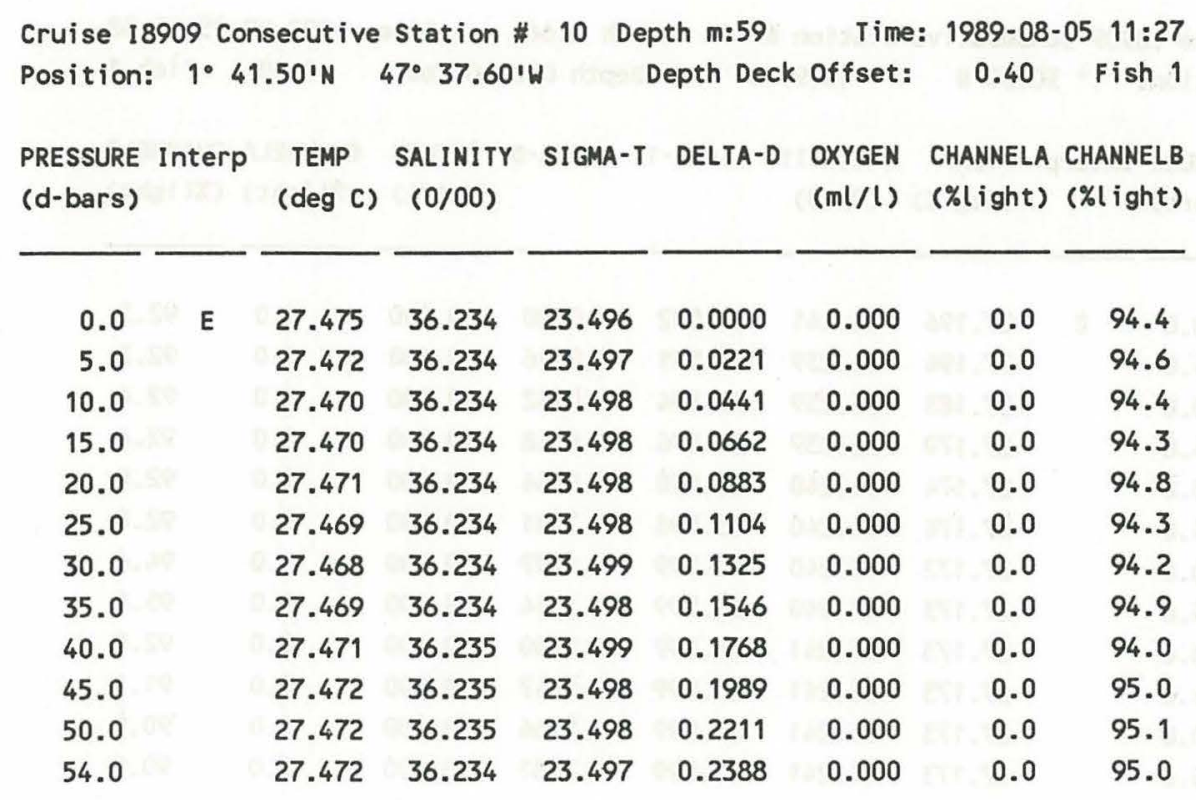
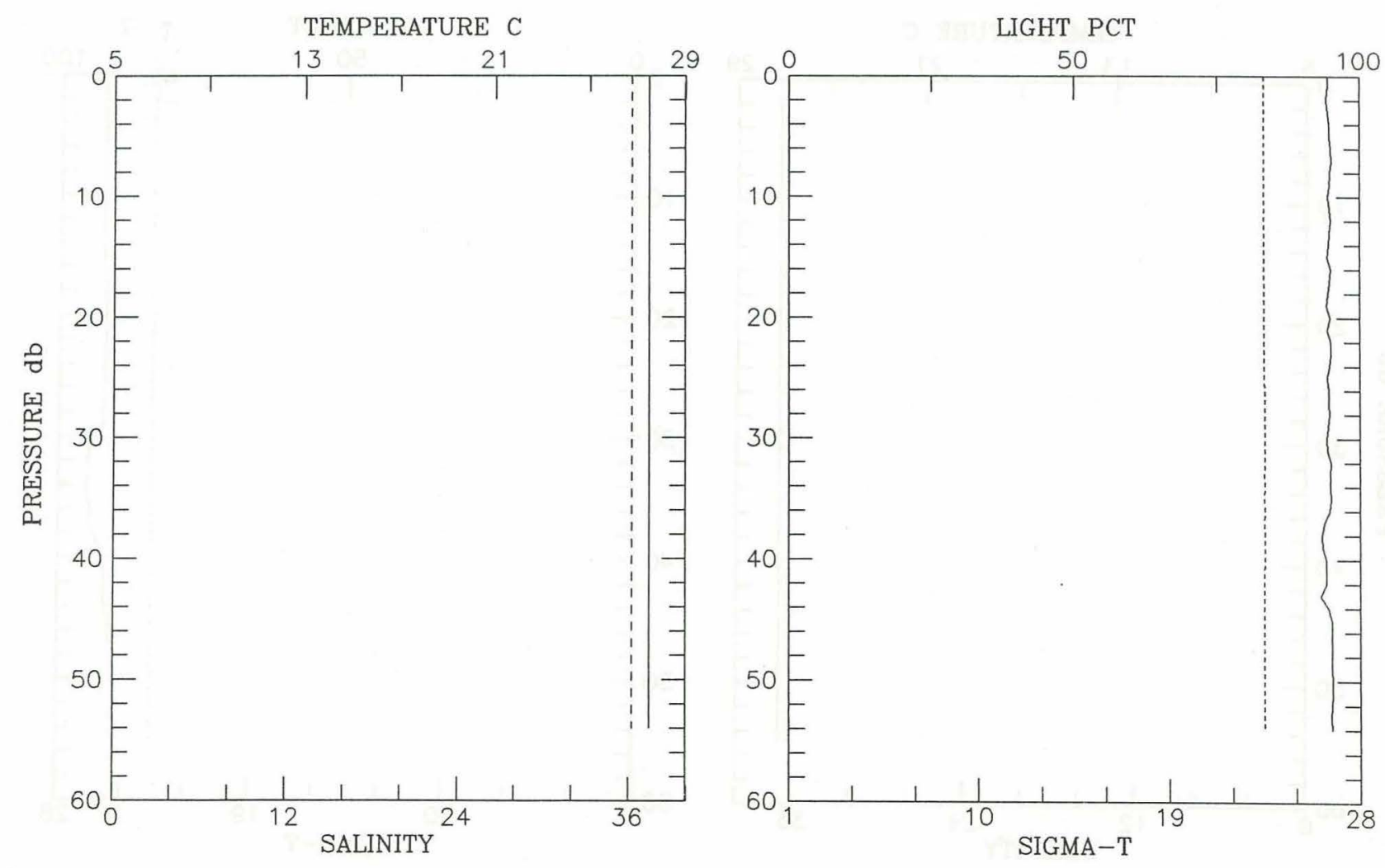


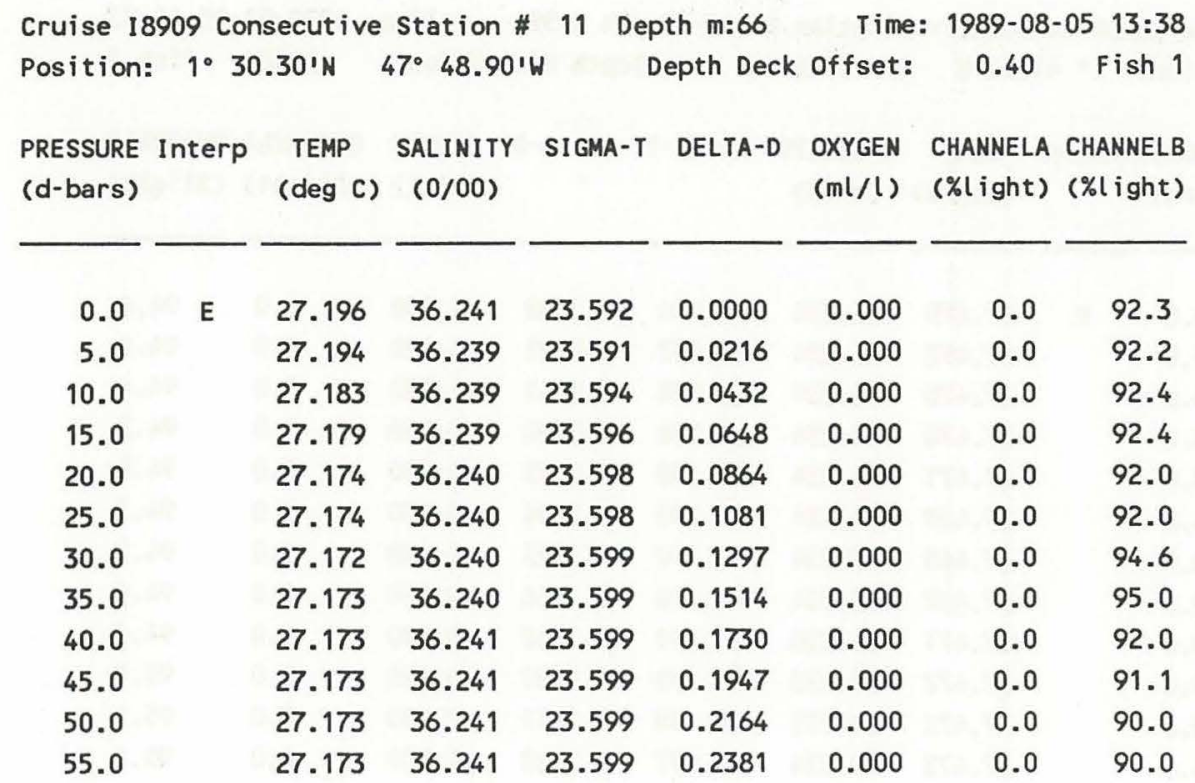
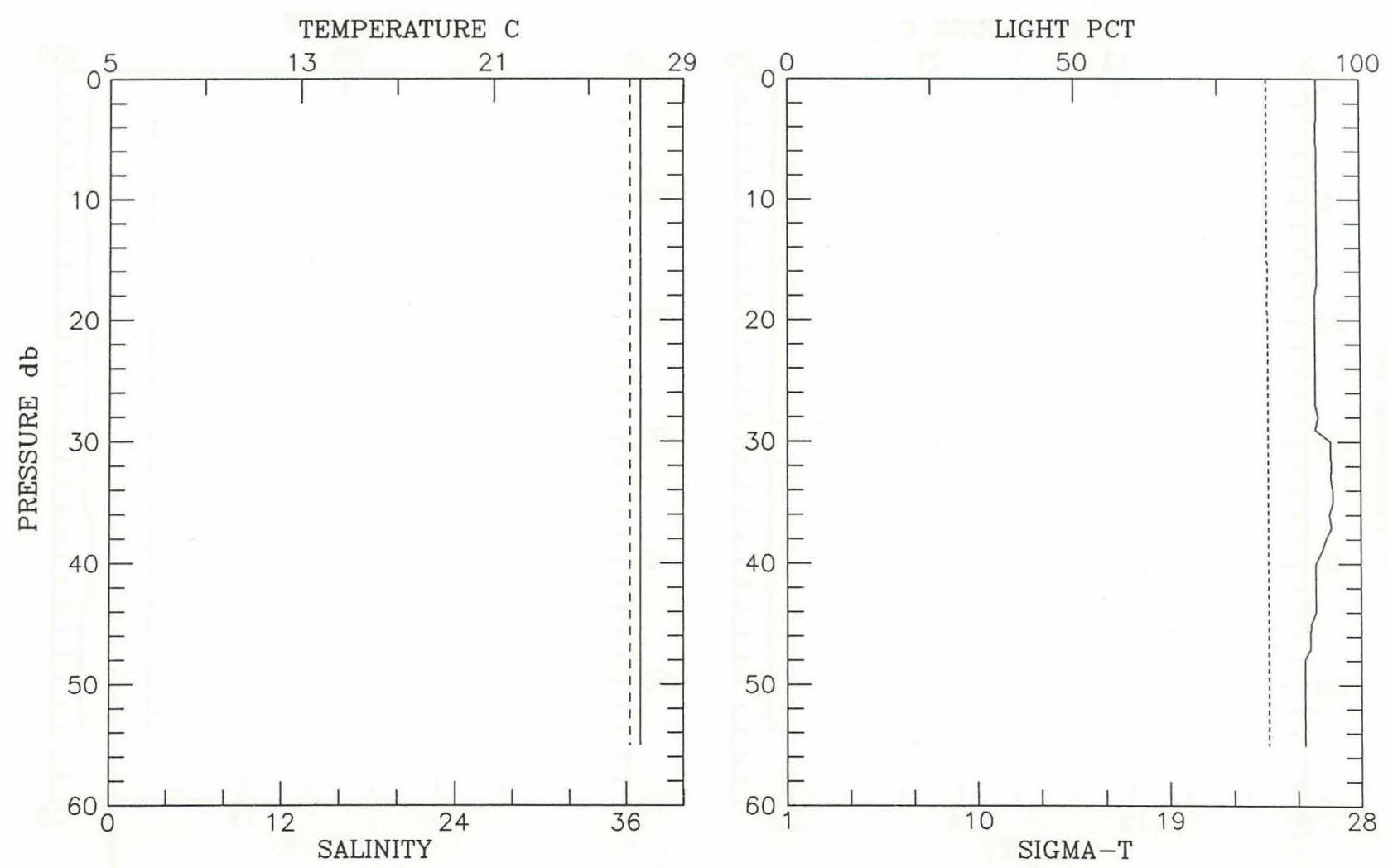


\begin{tabular}{|c|c|c|c|c|c|c|c|c|}
\hline Position: & $=1018$ & . 70 N & $\begin{array}{ll}48^{\circ} & 0.00^{\prime}\end{array}$ & & epth Deck & Offset: & 0.60 & Fish 1 \\
\hline $\begin{array}{l}\text { PRESSURE } \\
\text { (d-bars) }\end{array}$ & Interp & $\begin{array}{l}\text { TEMP } \\
(\operatorname{deg} C)\end{array}$ & $\begin{array}{l}\text { SALINITY } \\
(0 / 00)\end{array}$ & SI GMA-T & DELTA-D & $\begin{array}{l}\text { OXYGEN } \\
(\mathrm{ml} / \mathrm{l})\end{array}$ & $\begin{array}{l}\text { CHANNELA } \\
\text { (\%light) }\end{array}$ & $\begin{array}{l}\text { CHANNELB } \\
\text { (\%! ight) }\end{array}$ \\
\hline 0.0 & $E$ & 27.536 & 36.091 & 23.369 & 0.0000 & 0.000 & 0.0 & 90.1 \\
\hline 5.0 & & 27.535 & 36.090 & 23.368 & 0.0227 & 0.000 & 0.0 & 90.3 \\
\hline 10.0 & & 27.530 & 36.089 & 23.369 & 0.0454 & 0.000 & 0.0 & 90.4 \\
\hline 15.0 & & 27.497 & 36.089 & 23.380 & 0.0680 & 0.000 & 0.0 & 90.4 \\
\hline 20.0 & & 27.444 & 36.082 & 23.392 & 0.0906 & 0.000 & 0.0 & 90.3 \\
\hline 25.0 & & 27.406 & 36.081 & 23.404 & 0.1132 & 0.000 & 0.0 & 90.0 \\
\hline 30.0 & & 27.405 & 36.081 & 23.404 & 0.1358 & 0.000 & 0.0 & 89.8 \\
\hline 35.0 & & 27.406 & 36.081 & 23.404 & 0.1584 & 0.000 & 0.0 & 87.9 \\
\hline 40.0 & & 27.405 & 36.081 & 23.404 & 0.1810 & 0.000 & 0.0 & 87.8 \\
\hline 45.0 & & 27.404 & 36.082 & 23.405 & 0.2036 & 0.000 & 0.0 & 87.7 \\
\hline 48.0 & & 27.403 & 36.082 & 23.405 & 0.2171 & 0.000 & 0.0 & 87.5 \\
\hline
\end{tabular}
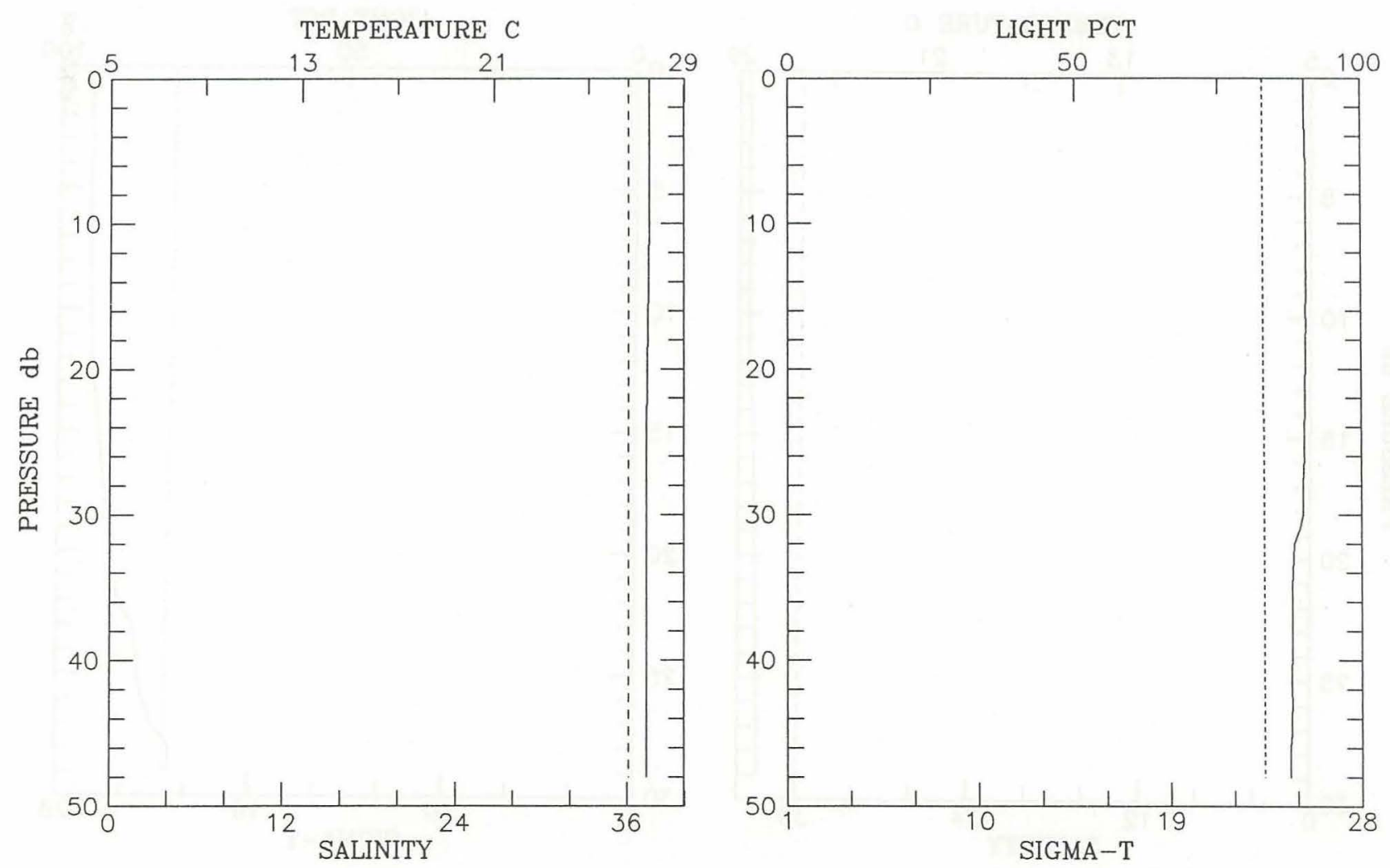
Cruise 18909 Consecutive Station \# 13 Depth m:34 Time: 1989-08-05 17:45 Position: $1^{\circ} 9.09^{\prime} \mathrm{N} \quad 48^{\circ} 11.90 \mathrm{~K}$ Depth Deck Offset: 0.00 Fish 1

\begin{tabular}{|c|c|c|c|c|c|c|c|c|}
\hline $\begin{array}{l}\text { PRESSURE } \\
\text { (d-bars) }\end{array}$ & Interp & $\begin{array}{c}\text { TEMP } \\
\text { (deg } \mathrm{C} \text { ) }\end{array}$ & $\begin{array}{c}\text { SALINITY } \\
(0 / 00)\end{array}$ & SIGMA-T & DELTA-D & $\begin{array}{l}\text { OXYGEN } \\
(m l / l)\end{array}$ & $\begin{array}{l}\text { CHANNELA } \\
\text { (\%light) }\end{array}$ & $\begin{array}{l}\text { CHANNELB } \\
\text { (\%light) }\end{array}$ \\
\hline 0.0 & E & 28.555 & 35.566 & 22.640 & 0.0000 & 0.000 & 0.0 & 94.5 \\
\hline 2.0 & & 28.554 & 35.566 & 22.640 & 0.0105 & 0.000 & 0.0 & 94.5 \\
\hline 4.0 & & 28.553 & 35.566 & 22.640 & 0.0209 & 0.000 & 0.0 & 94.5 \\
\hline 6.0 & & 28.547 & 35.565 & 22.641 & 0.0314 & 0.000 & 0.0 & 94.5 \\
\hline 8.0 & & 28.544 & 35.564 & 22.642 & 0.0418 & 0.000 & 0.0 & 94.5 \\
\hline 10.0 & & 28.540 & 35.561 & 22.641 & 0.0523 & 0.000 & 0.0 & 94.4 \\
\hline 12.0 & & 28.489 & 35.573 & 22.667 & 0.0628 & 0.000 & 0.0 & 94.2 \\
\hline 14.0 & & 28.400 & 35.625 & 22.735 & 0.0731 & 0.000 & 0.0 & 93.3 \\
\hline 16.0 & & 28.341 & 35.665 & 22.785 & 0.0834 & 0.000 & 0.0 & 92.6 \\
\hline 18.0 & & 28.316 & 35.689 & 22.811 & 0.0935 & 0.000 & 0.0 & 91.7 \\
\hline 20.0 & & 28.274 & 35.726 & 22.853 & 0.1036 & 0.000 & 0.0 & 90.8 \\
\hline 22.0 & & 28.253 & 35.749 & 22.877 & 0.1137 & 0.000 & 0.0 & 89.3 \\
\hline 24.0 & & 28.201 & 35.802 & 22.934 & 0.1236 & 0.000 & 0.0 & 86.0 \\
\hline 26.0 & & 28.188 & 35.815 & 22.948 & 0.1336 & 0.000 & 0.0 & 85.0 \\
\hline 28.0 & & 28.168 & 35.834 & 22.969 & 0.1434 & 0.000 & 0.0 & 80.4 \\
\hline 29.0 & & 28.168 & 35.834 & 22.969 & 0.1484 & 0.000 & 0.0 & 80.7 \\
\hline
\end{tabular}
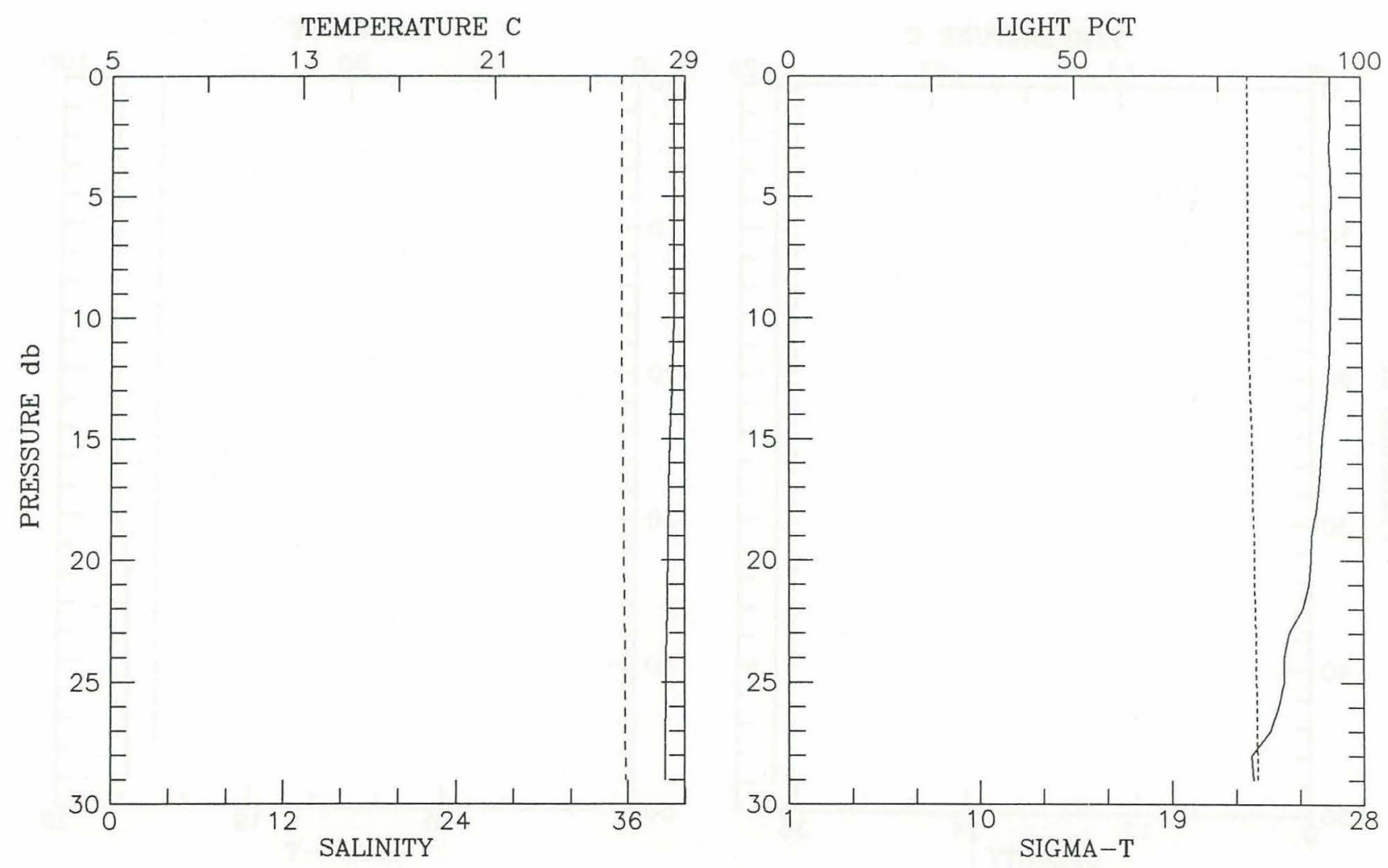


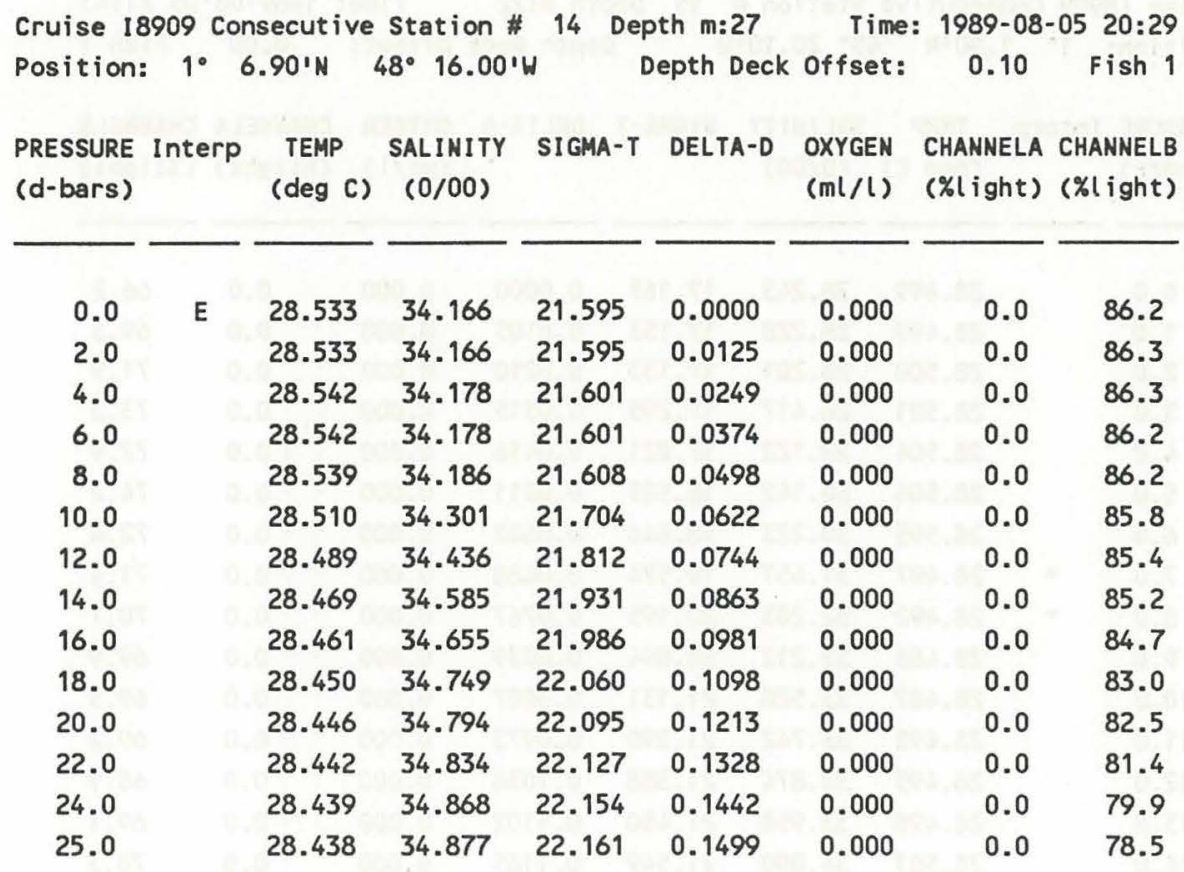
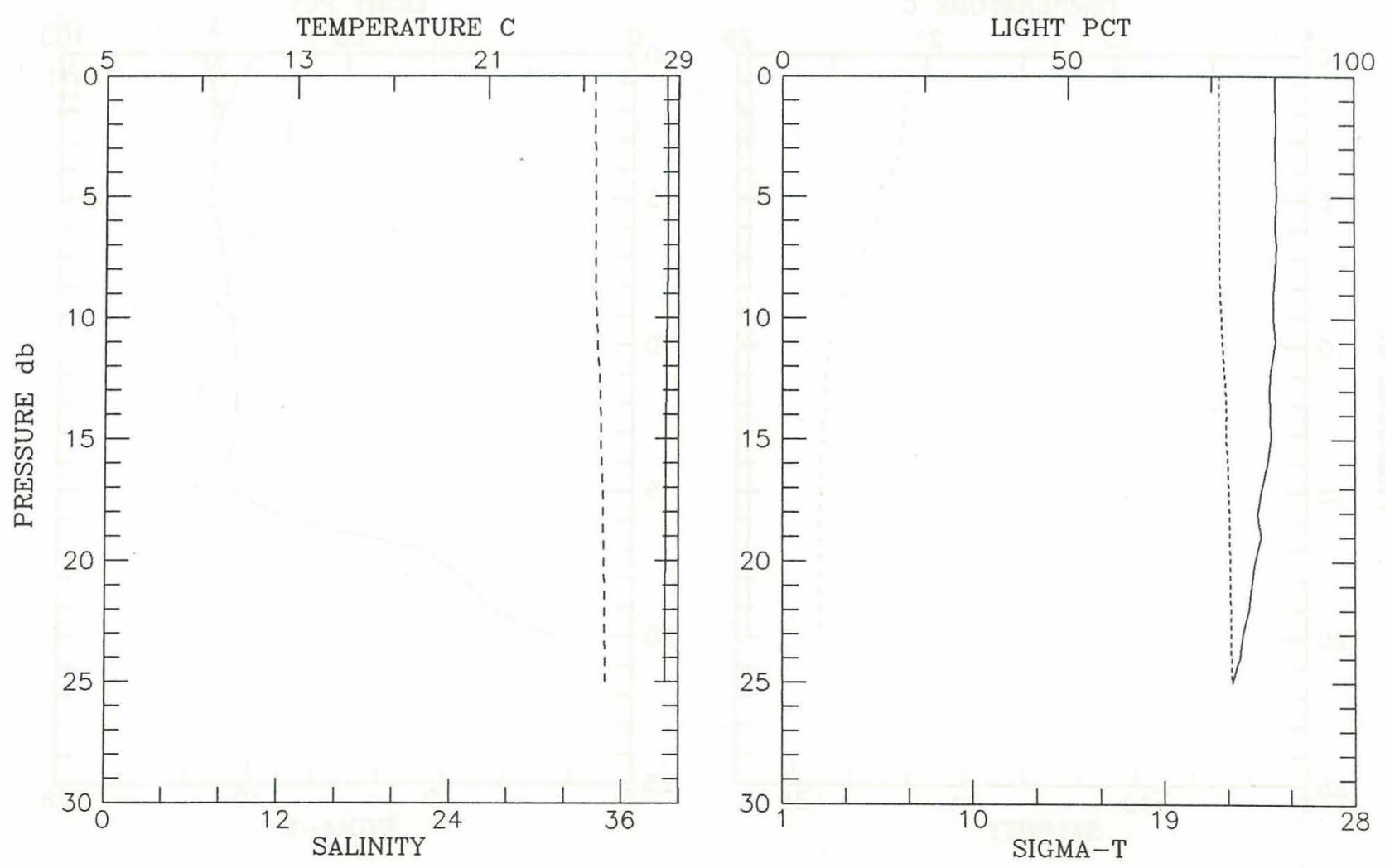


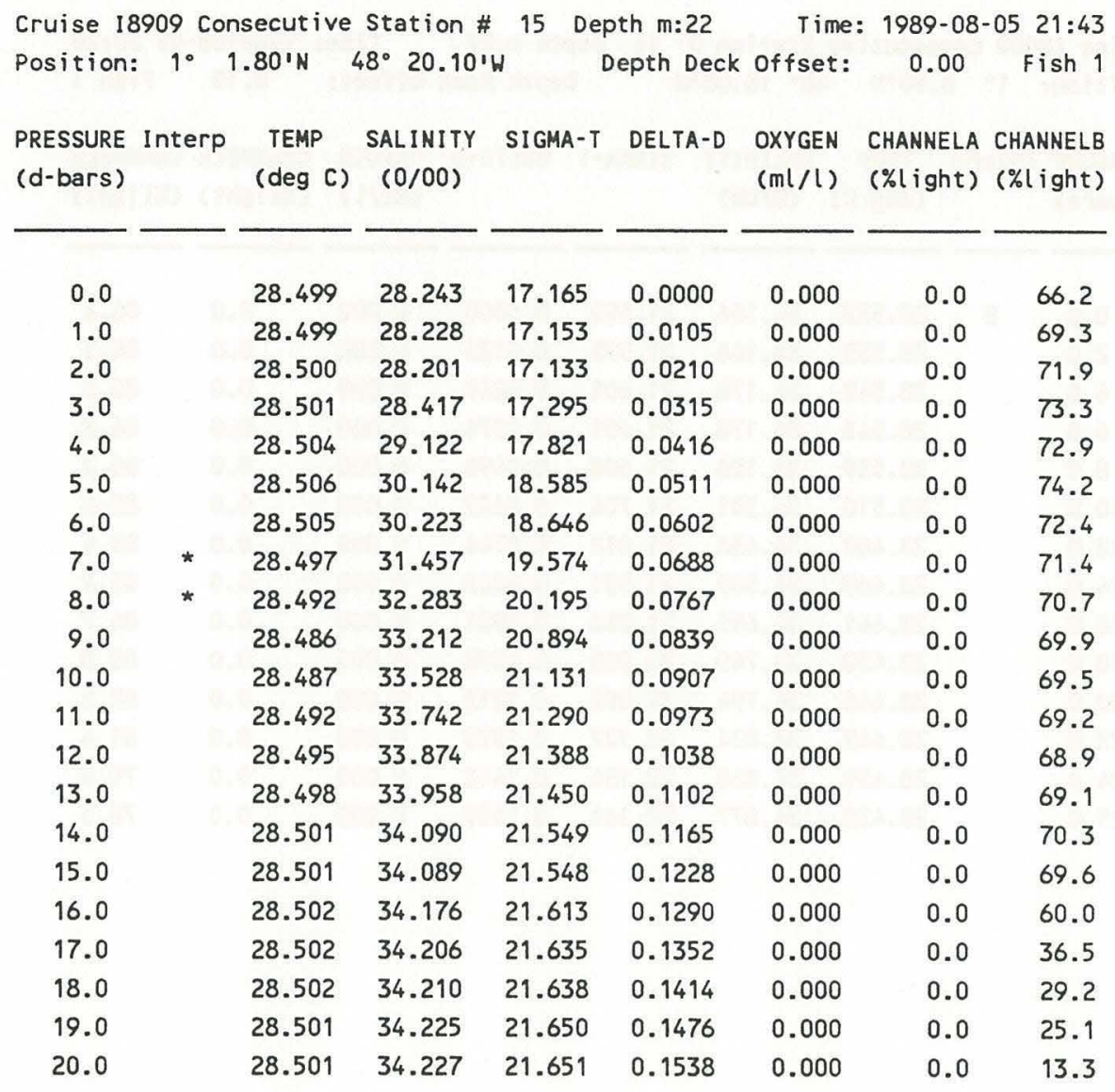
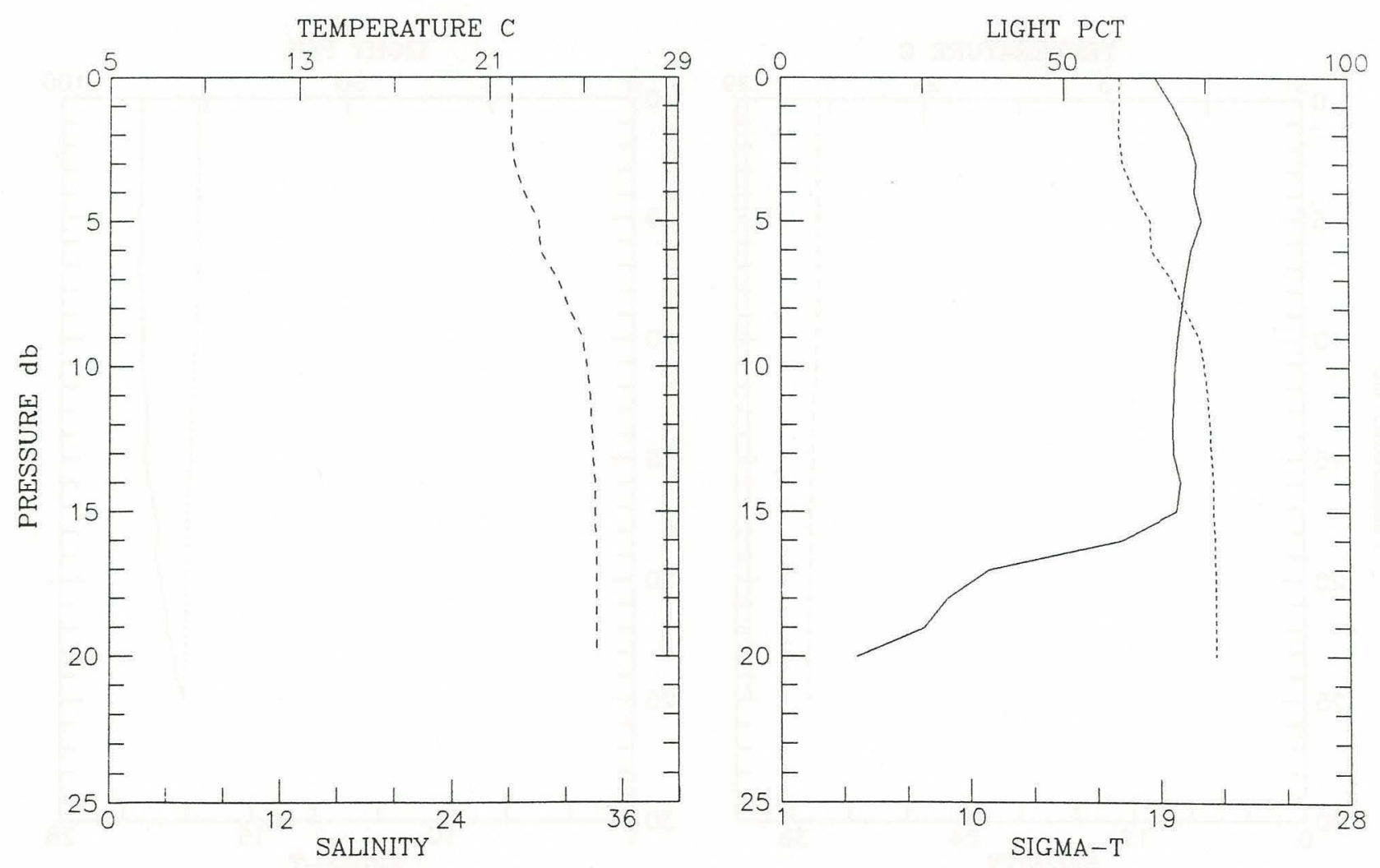


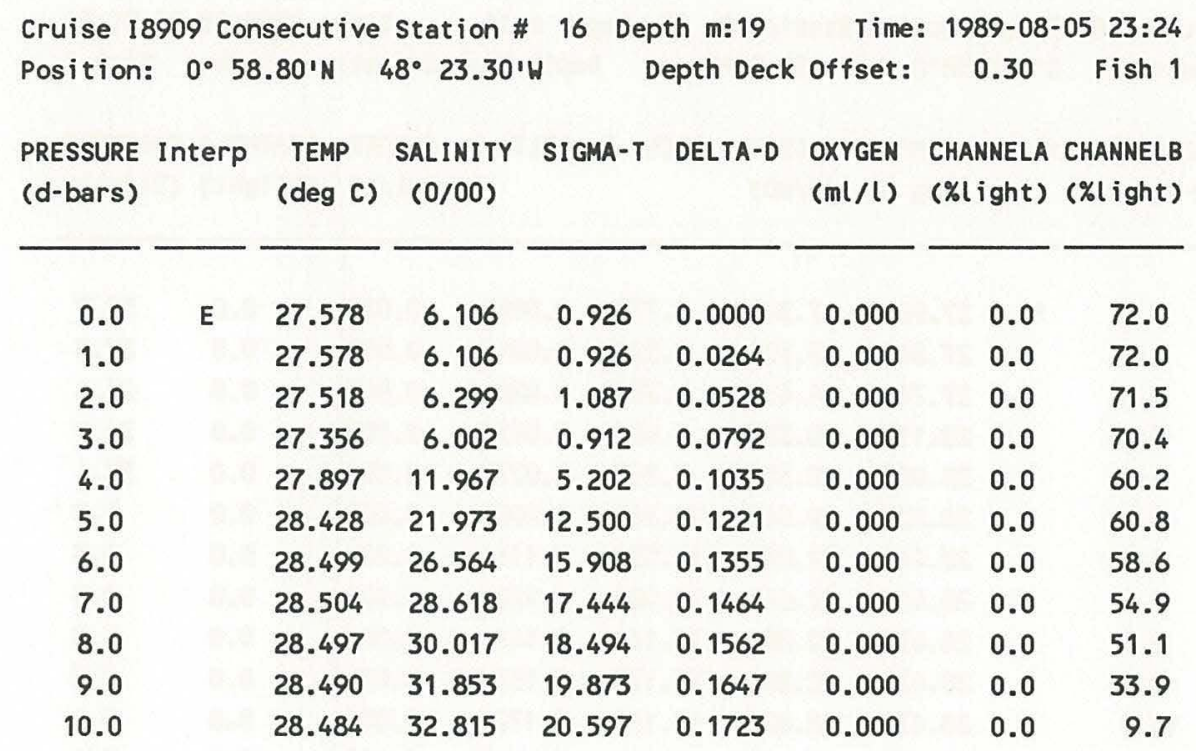
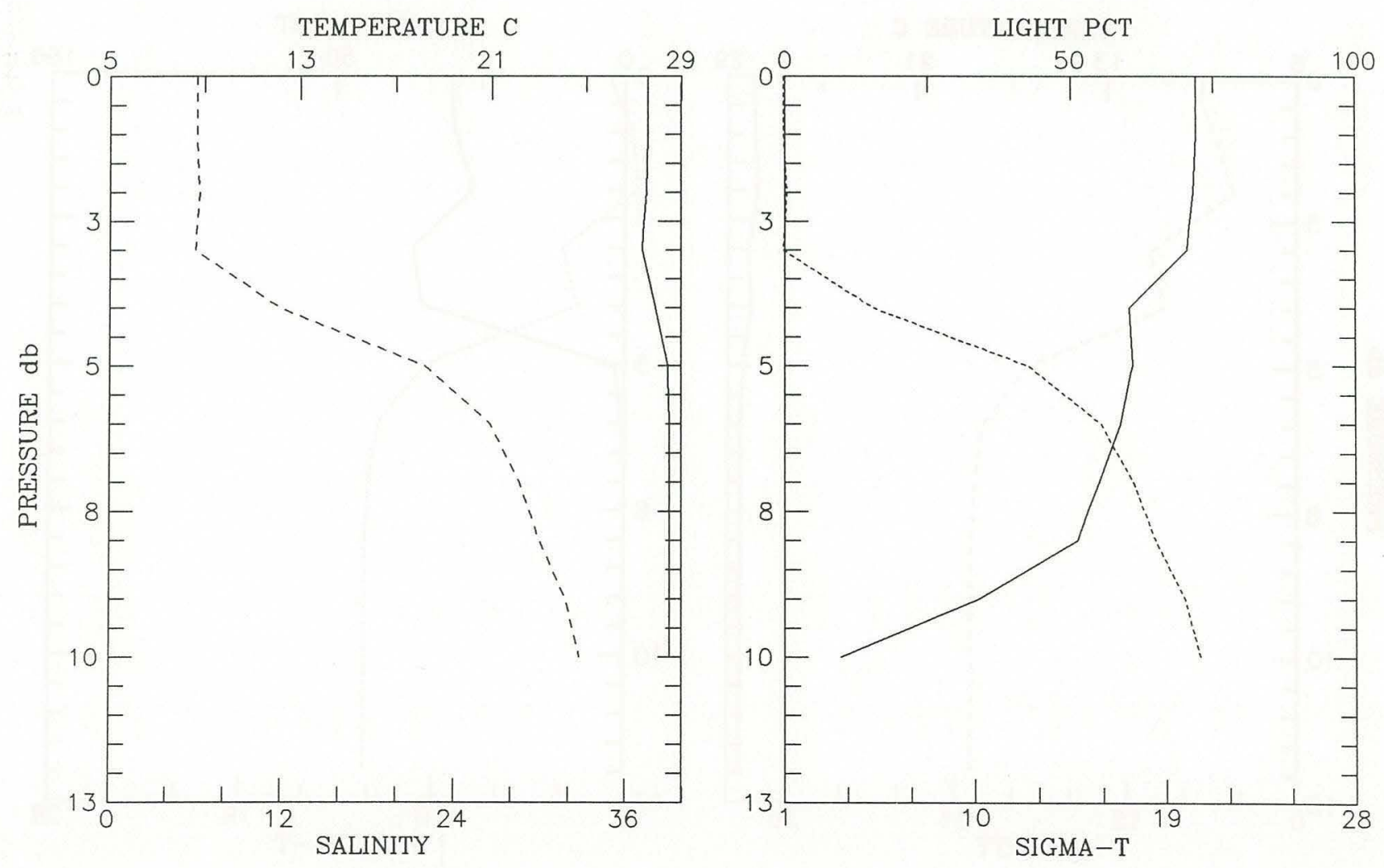


\begin{tabular}{|c|c|c|c|c|c|c|c|c|}
\hline $\begin{array}{l}\text { Cruise } 18 \\
\text { Position: }\end{array}$ & $\begin{array}{r}3909 \text { Con } \\
0^{\circ} 43\end{array}$ & .50'N & $\begin{array}{l}\text { Station } \\
48^{\circ} 31.90^{\prime}\end{array}$ & 170 & epth Deck & $\begin{array}{l}\text { Time: } \\
\text { Offset: }\end{array}$ & 0.00 & Fish 1 \\
\hline $\begin{array}{l}\text { PRESSURE } \\
\text { (d-bars) }\end{array}$ & Interp & $\begin{array}{c}\text { TEMP } \\
(\operatorname{deg} C)\end{array}$ & $\begin{array}{c}\text { SALINITY } \\
(0 / 00)\end{array}$ & SIGMA-T & DELTA-D & $\begin{array}{l}\text { OXYGEN } \\
(m l / l)\end{array}$ & $\begin{array}{l}\text { CHANNELA } \\
\text { (\%light) }\end{array}$ & $\begin{array}{l}\text { CHANNELB } \\
\text { (\%light) }\end{array}$ \\
\hline 0.0 & * & 27.941 & 7.380 & 1.770 & 0.0000 & 0.000 & 0.0 & 30.7 \\
\hline 1.0 & & 27.841 & 5.759 & 0.591 & 0.0011 & 0.000 & 0.0 & 29.8 \\
\hline 2.0 & & 27.744 & 4.456 & 0.354 & 0.0284 & 0.000 & 0.0 & 26.4 \\
\hline 3.0 & & 28.117 & 10.358 & 3.937 & 0.0539 & 0.000 & 0.0 & 36.9 \\
\hline 4.0 & & 28.029 & 9.350 & 3.213 & 0.0778 & 0.000 & 0.0 & 35.4 \\
\hline 5.0 & & 28.338 & 19.043 & 10.341 & 0.0984 & 0.000 & 0.0 & 1.9 \\
\hline 6.0 & & 28.448 & 22.097 & 12.586 & 0.1144 & 0.000 & 0.0 & 0.3 \\
\hline 7.0 & & 28.462 & 22.642 & 12.989 & 0.1292 & 0.000 & 0.0 & 0.0 \\
\hline 8.0 & & 28.471 & 22.880 & 13.164 & 0.1436 & 0.000 & 0.0 & 0.0 \\
\hline 9.0 & & 28.473 & 22.894 & 13.173 & 0.1580 & 0.000 & 0.0 & 0.0 \\
\hline 10.0 & & 28.471 & 22.887 & 13.169 & 0.1724 & 0.000 & 0.0 & 0.0 \\
\hline 11.0 & & 28.472 & 22.882 & 13.165 & 0.1868 & 0.000 & 0.0 & 0.0 \\
\hline 12.0 & & 28.473 & 22.884 & 13.166 & 0.2011 & 0.000 & 0.0 & 0.0 \\
\hline
\end{tabular}
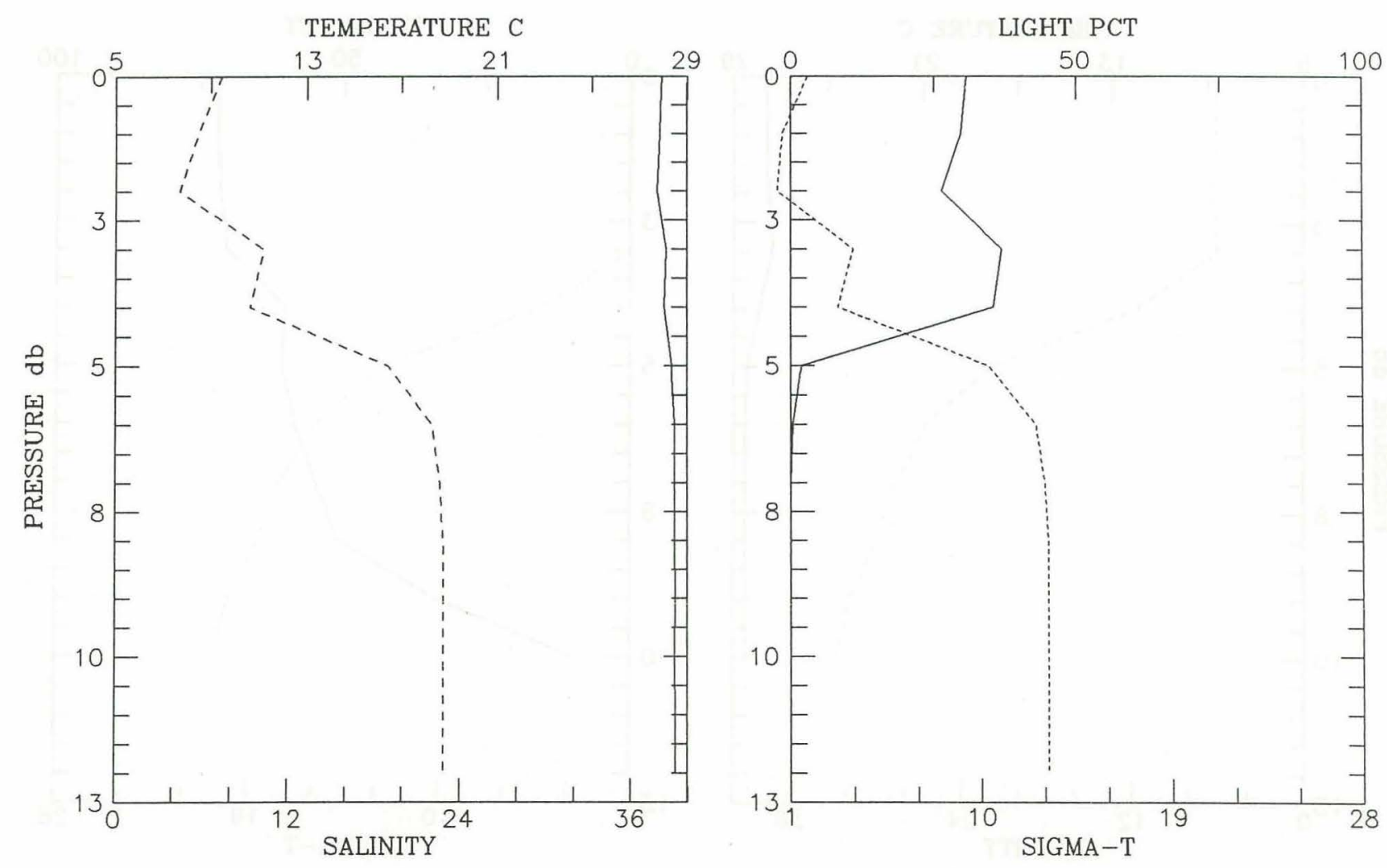


\begin{tabular}{|c|c|c|c|c|c|c|c|c|}
\hline $\begin{array}{l}\text { Cruise } 18 \\
\text { Position: }\end{array}$ & $\begin{array}{r}3909 \text { Con } \\
1^{\circ} 28\end{array}$ & 60 'N 4 & $\begin{array}{l}\text { Station \# } \\
49^{\circ} 23.90 \text { I }\end{array}$ & 18 Dep & $\begin{array}{l}\text { th } \mathrm{m}: 11 \\
\text { epth Deck }\end{array}$ & $\begin{array}{r}\text { Time: } \\
\text { Offset: }\end{array}$ & $\begin{array}{c}1989-08 \\
0.00\end{array}$ & $\begin{array}{r}-0609: 27 \\
\text { Fish } 1\end{array}$ \\
\hline $\begin{array}{l}\text { PRESSURE } \\
\text { (d-bars) }\end{array}$ & Interp & $\begin{array}{l}\text { TEMP } \\
(\operatorname{deg} C)\end{array}$ & $\begin{array}{l}\text { SALINITY } \\
(0 / 00)\end{array}$ & SIGMA-T & DELTA-D & $\begin{array}{l}\text { OXYGEN } \\
(\mathrm{ml} / \mathrm{l})\end{array}$ & $\begin{array}{l}\text { CHANNELA } \\
\text { (\%light) }\end{array}$ & $\begin{array}{l}\text { CHANNELB } \\
\text { (\%light) }\end{array}$ \\
\hline 0.0 & $E$ & 27.924 & 0.034 & 0.000 & 0.0000 & 0.000 & 0.0 & 0.0 \\
\hline 1.0 & & 27.924 & 0.034 & 0.000 & 0.0311 & 0.000 & 0.0 & 0.0 \\
\hline 2.0 & & 27.970 & 0.034 & 0.000 & 0.0622 & 0.000 & 0.0 & 0.0 \\
\hline 3.0 & & 27.958 & 0.039 & 0.000 & 0.0933 & 0.000 & 0.0 & 0.0 \\
\hline 4.0 & & 27.971 & 0.036 & 0.000 & 0.1244 & 0.000 & 0.0 & 0.0 \\
\hline 5.0 & * & 27.976 & 0.036 & 0.000 & 0.1555 & 0.000 & 0.0 & 0.0 \\
\hline 6.0 & * & 27.979 & 0.037 & 0.000 & 0.1866 & 0.000 & 0.0 & 0.0 \\
\hline 7.0 & & 27.984 & 0.037 & 0.000 & 0.2177 & 0.000 & 0.0 & 0.0 \\
\hline 8.0 & & 27.983 & 0.037 & 0.000 & 0.2489 & 0.000 & 0.0 & 0.0 \\
\hline
\end{tabular}
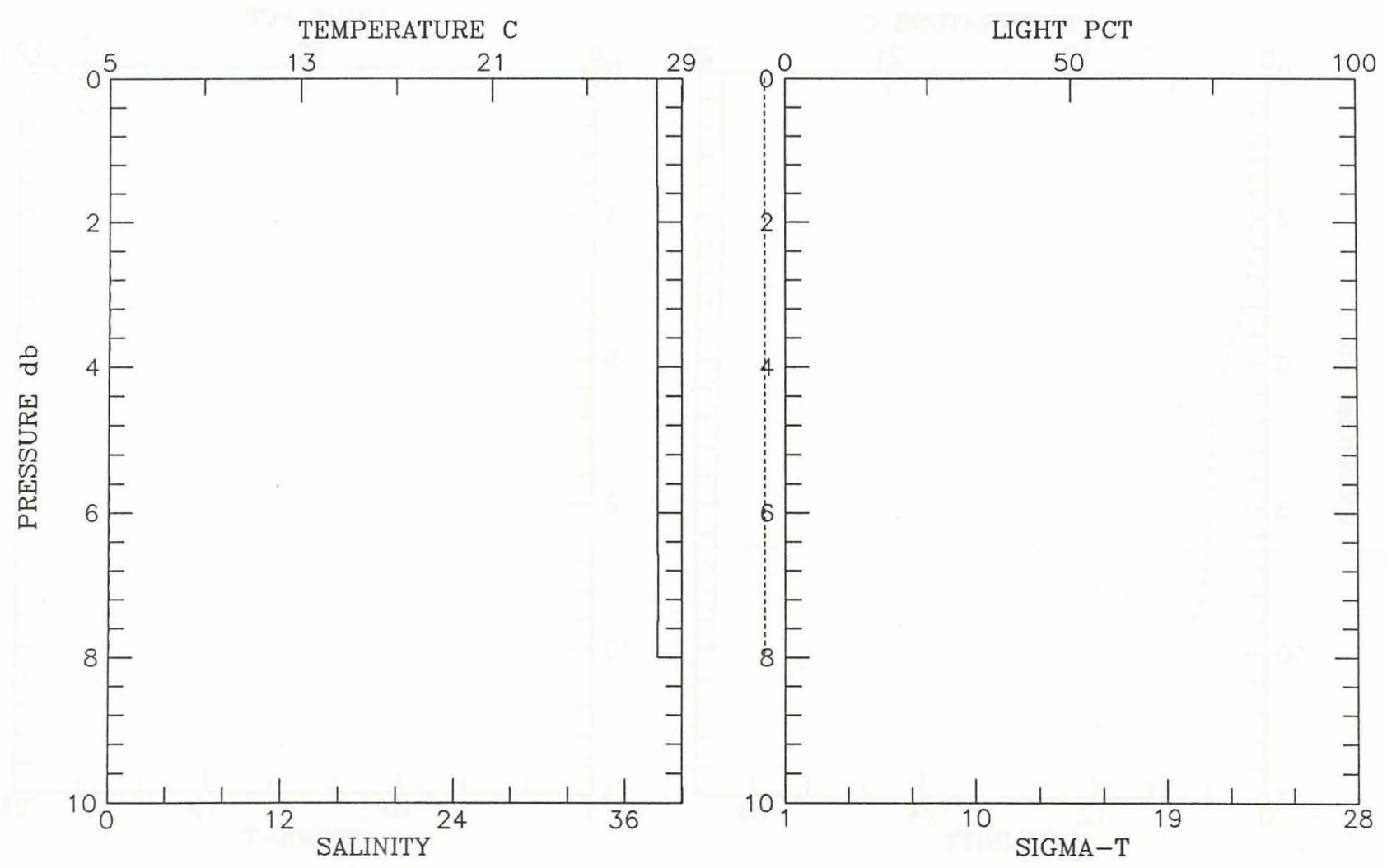


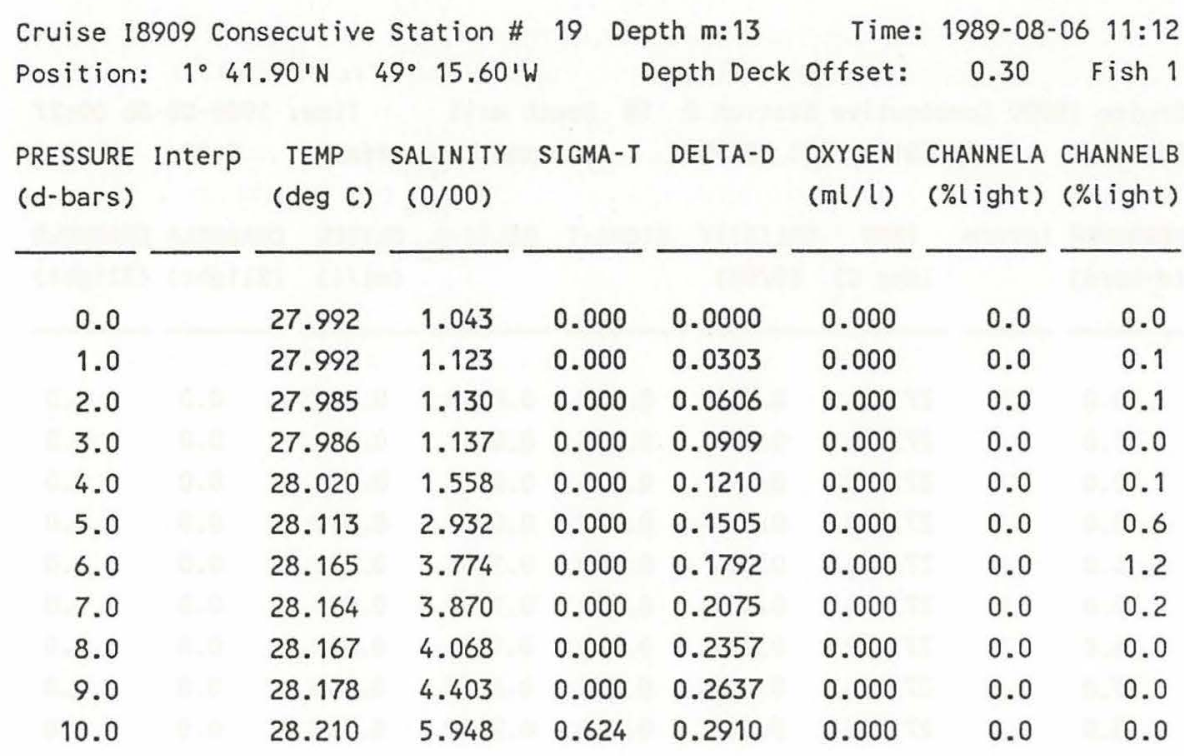
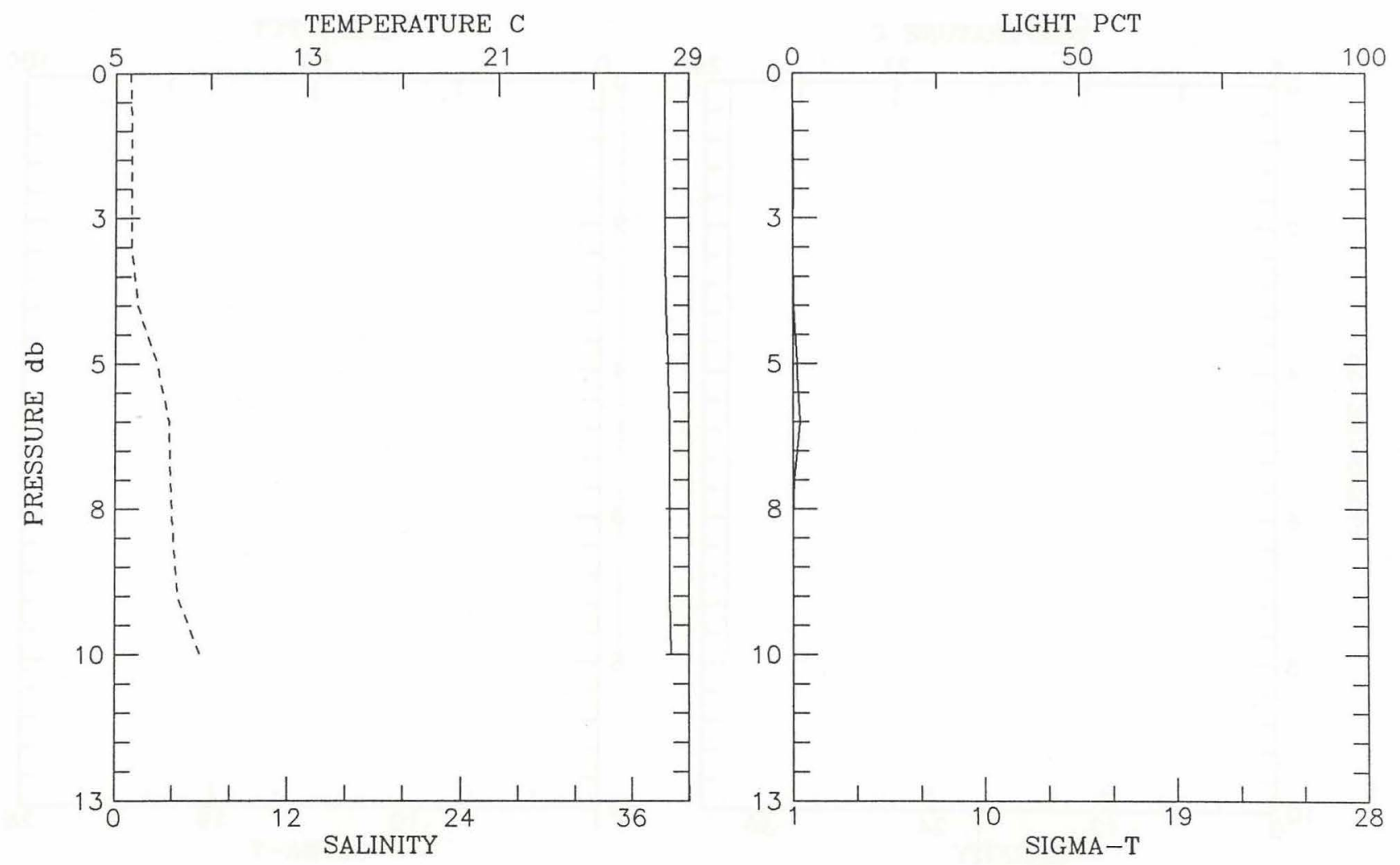


\begin{tabular}{|c|c|c|c|c|c|c|c|c|}
\hline \multirow{2}{*}{$\begin{array}{l}\text { Cruise } 18 \\
\text { Position: } \\
\text { PRESSURE } \\
\text { (d-bars) }\end{array}$} & \multicolumn{2}{|c|}{$\begin{array}{l}8909 \text { Consecutive } \\
\text { 1. } 52.90 \mathrm{~N}\end{array}$} & $\begin{array}{l}\text { Station } \\
48^{\circ} 58.90^{\prime} \mathrm{H}\end{array}$ & $H$ & $\begin{array}{l}\text { epth m: } 14 \\
\text { epth Deck }\end{array}$ & $\begin{array}{l}\text { Time: } \\
\text { offset: }\end{array}$ & $\begin{array}{c}1989.08 \\
0.10\end{array}$ & $\begin{array}{r}.06 \quad 13: 21 \\
\text { Fish } 1\end{array}$ \\
\hline & Interp & $\begin{array}{l}\text { TEMP } \\
\text { (deg C) }\end{array}$ & $\begin{array}{l}\text { SALINITY } \\
(0 / 00)\end{array}$ & SIGMA-T & DELTA-D & $\begin{array}{l}\text { OXYGEN } \\
(m / / l)\end{array}$ & $\begin{array}{l}\text { CHANNELA } \\
\text { (\%tight) }\end{array}$ & $\begin{array}{l}\text { CHANNELB } \\
\text { ( } \% \text { light) }\end{array}$ \\
\hline 0.0 & E & 28.158 & 5.877 & 0.586 & 0.0000 & 0.000 & 0.0 & 54.8 \\
\hline 1.0 & & 28.158 & 5.877 & 0.586 & 0.0268 & 0.000 & 0.0 & 54.8 \\
\hline 2.0 & & 28.202 & 9.655 & 3.388 & 0.0522 & 0.000 & 0.0 & 56.1 \\
\hline 3.0 & & 28.277 & 15.171 & 7.475 & 0.0741 & 0.000 & 0.0 & 57.5 \\
\hline 4.0 & & 28.303 & 19.151 & 10.433 & 0.0926 & 0.000 & 0.0 & 58.4 \\
\hline 5.0 & & 28.287 & 27.799 & 16.900 & 0.1065 & 0.000 & 0.0 & 43.0 \\
\hline 6.0 & & 28.257 & 32.306 & 20.289 & 0.1157 & 0.000 & 0.0 & 15.8 \\
\hline 7.0 & & 28.246 & 33.121 & 20.904 & 0.1228 & 0.000 & 0.0 & 6.2 \\
\hline 8.0 & & 28.243 & 33.371 & 21.093 & 0.1296 & 0.000 & 0.0 & 1.7 \\
\hline 9.0 & & 28.241 & 33.442 & 21.147 & 0.1363 & 0.000 & 0.0 & 0.4 \\
\hline 10.0 & & 28.249 & 33.452 & 21.155 & 0.1430 & 0.000 & 0.0 & 0.2 \\
\hline 11.0 & & 28.240 & 33.468 & 21.167 & 0.1496 & 0.000 & 0.0 & 0.1 \\
\hline
\end{tabular}
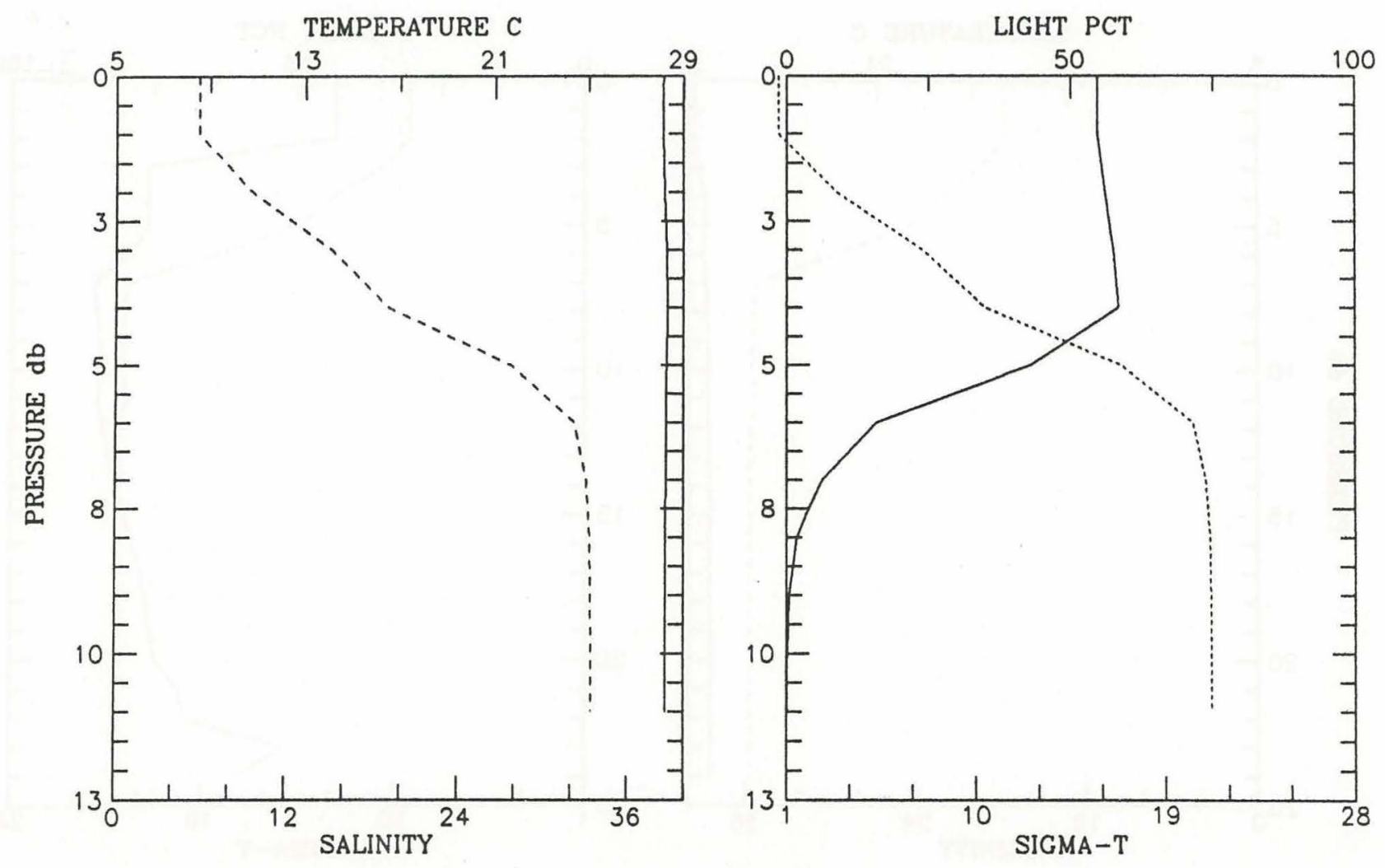


\begin{tabular}{|c|c|c|c|c|c|c|c|c|}
\hline Position: & $2^{\circ}$ & $.30^{\prime} \mathrm{N}$ & $48^{\circ} 49.00^{\prime}$ & & epth Deck & Offset: & 0.40 & Fish 1 \\
\hline $\begin{array}{l}\text { PRESSURE } \\
\text { (d-bars) }\end{array}$ & Interp & $\begin{array}{c}\text { TEMP } \\
(\operatorname{deg} C)\end{array}$ & $\begin{array}{l}\text { SALINITY } \\
(0 / 00)\end{array}$ & SIGMA-T & DELTA-D & $\begin{array}{l}\text { OXYGEN } \\
(\mathrm{ml} / \mathrm{l})\end{array}$ & $\begin{array}{l}\text { CHANNELA } \\
\text { (\%light) }\end{array}$ & $\begin{array}{l}\text { CHANNELB } \\
\text { (\%light) }\end{array}$ \\
\hline 0.0 & $E$ & 28.733 & 17.761 & 9.263 & 0.0000 & 0.000 & 0.0 & 43.5 \\
\hline 2.0 & & 28.733 & 17.760 & 9.262 & 0.0364 & 0.000 & 0.0 & 43.5 \\
\hline 4.0 & & 28.227 & 22.311 & 12.815 & 0.0697 & 0.000 & 0.0 & 76.1 \\
\hline 6.0 & & 28.364 & 29.515 & 18.161 & 0.0950 & 0.000 & 0.0 & 79.1 \\
\hline 8.0 & & 28.186 & 35.445 & 22.670 & 0.1077 & 0.000 & 0.0 & 85.1 \\
\hline 10.0 & & 28.149 & 35.518 & 22.738 & 0.1180 & 0.000 & 0.0 & 84.6 \\
\hline 12.0 & & 28.113 & 35.591 & 22.804 & 0.1285 & 0.000 & 0.0 & 84.0 \\
\hline 14.0 & & 28.096 & 35.629 & 22.839 & 0.1386 & 0.000 & 0.0 & 81.9 \\
\hline 16.0 & & 28.077 & 35.661 & 22.869 & 0.1487 & 0.000 & 0.0 & 78.8 \\
\hline 18.0 & & 28.075 & 35.668 & 22.875 & 0.1587 & 0.000 & 0.0 & 76.3 \\
\hline 20.0 & & 28.069 & 35.674 & 22.881 & 0.1687 & 0.000 & 0.0 & 74.7 \\
\hline 22.0 & & 28.068 & 35.679 & 22.885 & 0.1787 & 0.000 & 0.0 & 69.6 \\
\hline 24.0 & & 28.063 & 35.682 & 22.889 & 0.1887 & 0.000 & 0.0 & 60.6 \\
\hline
\end{tabular}
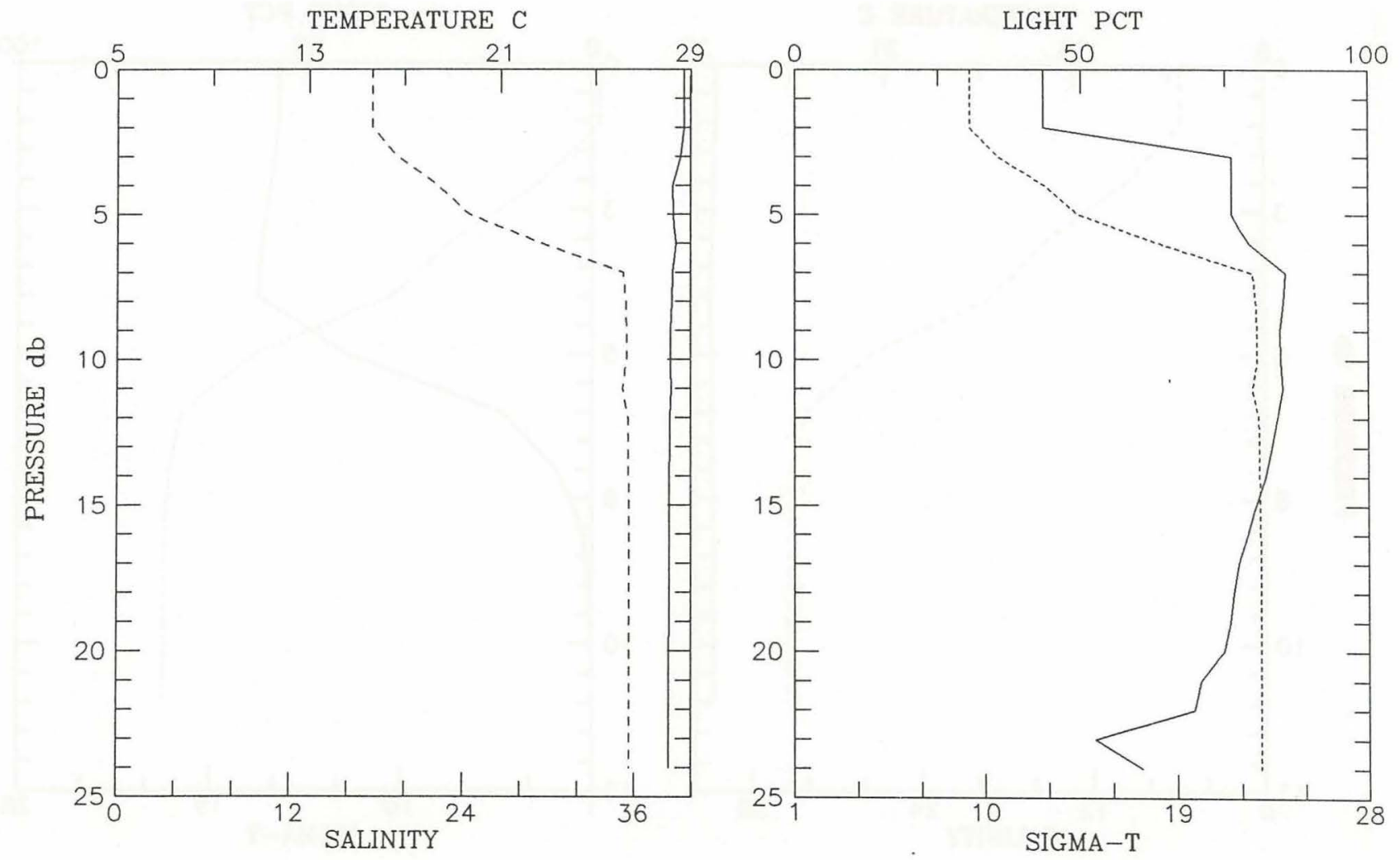


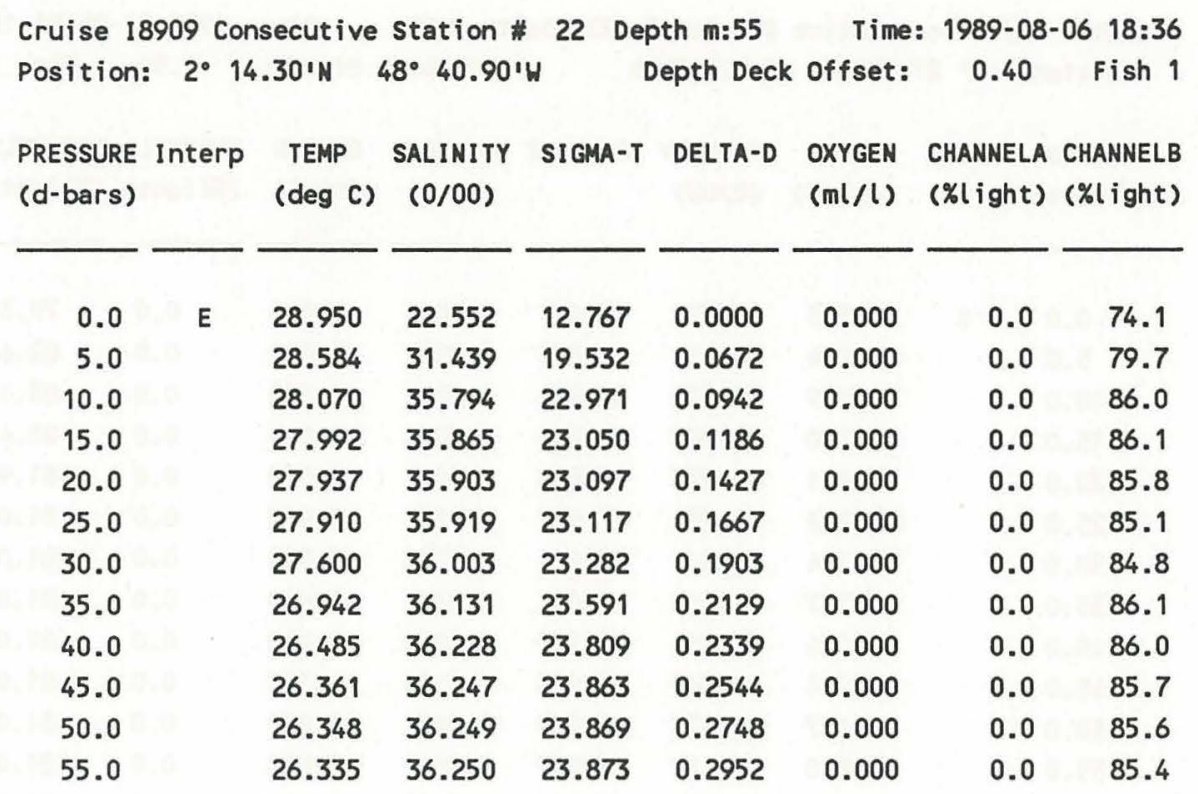
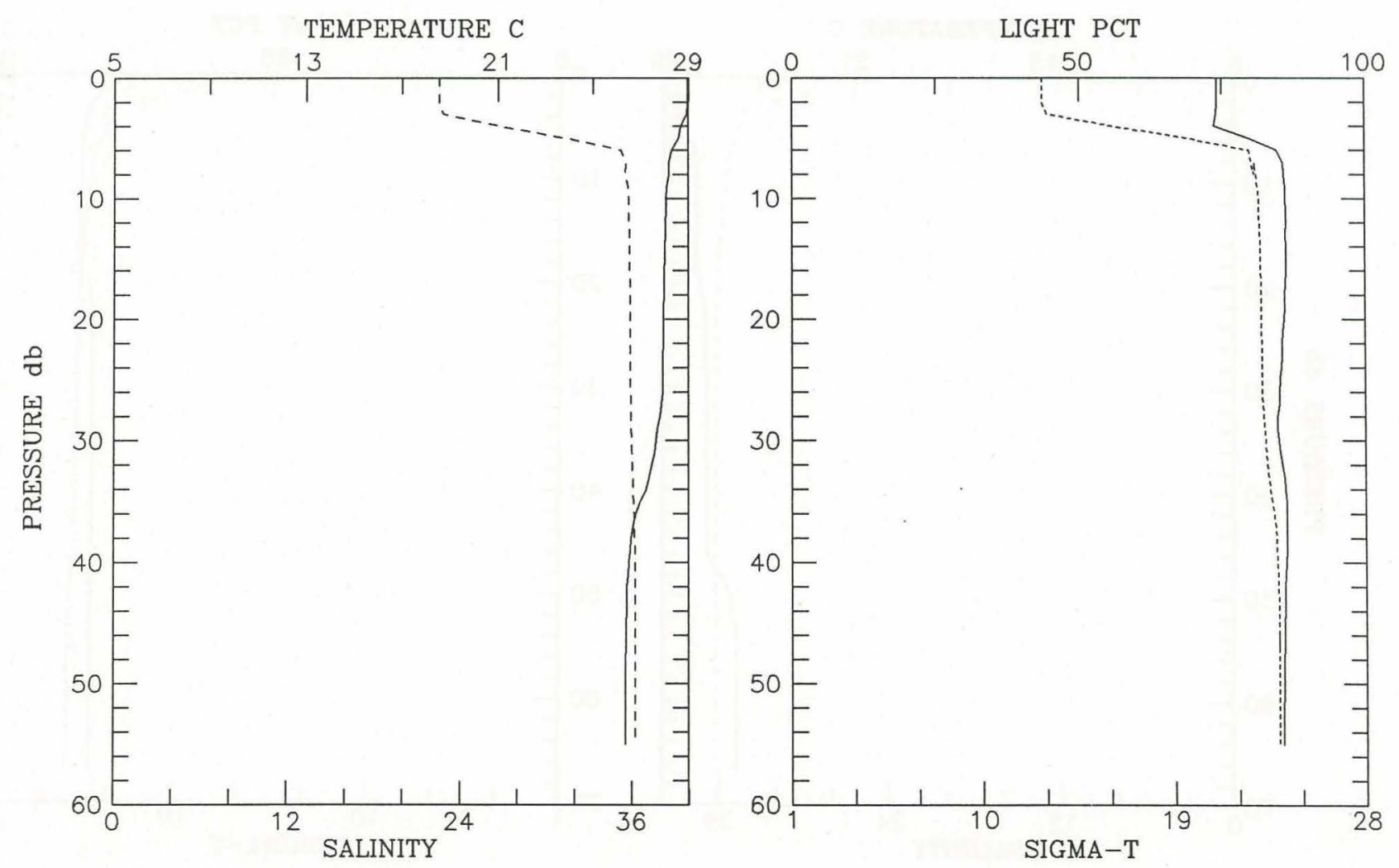


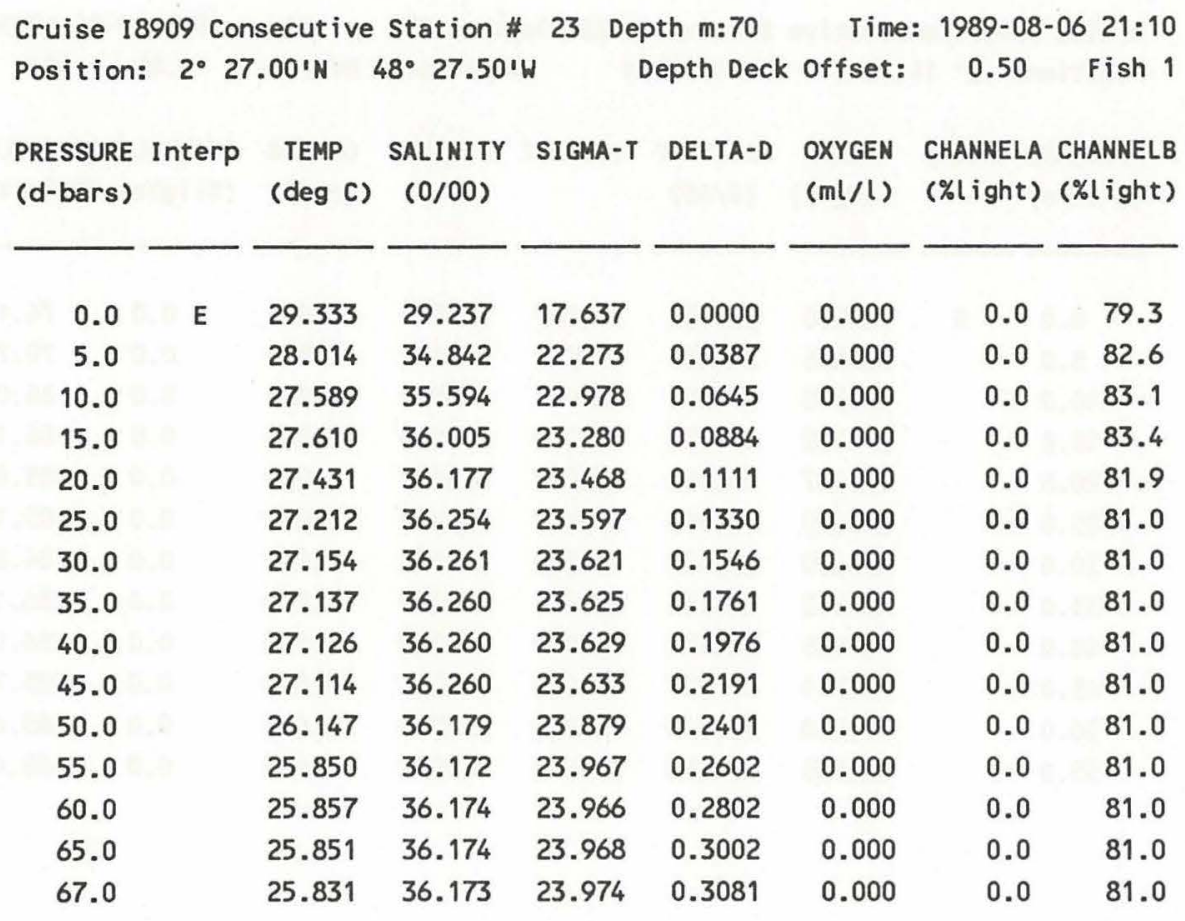
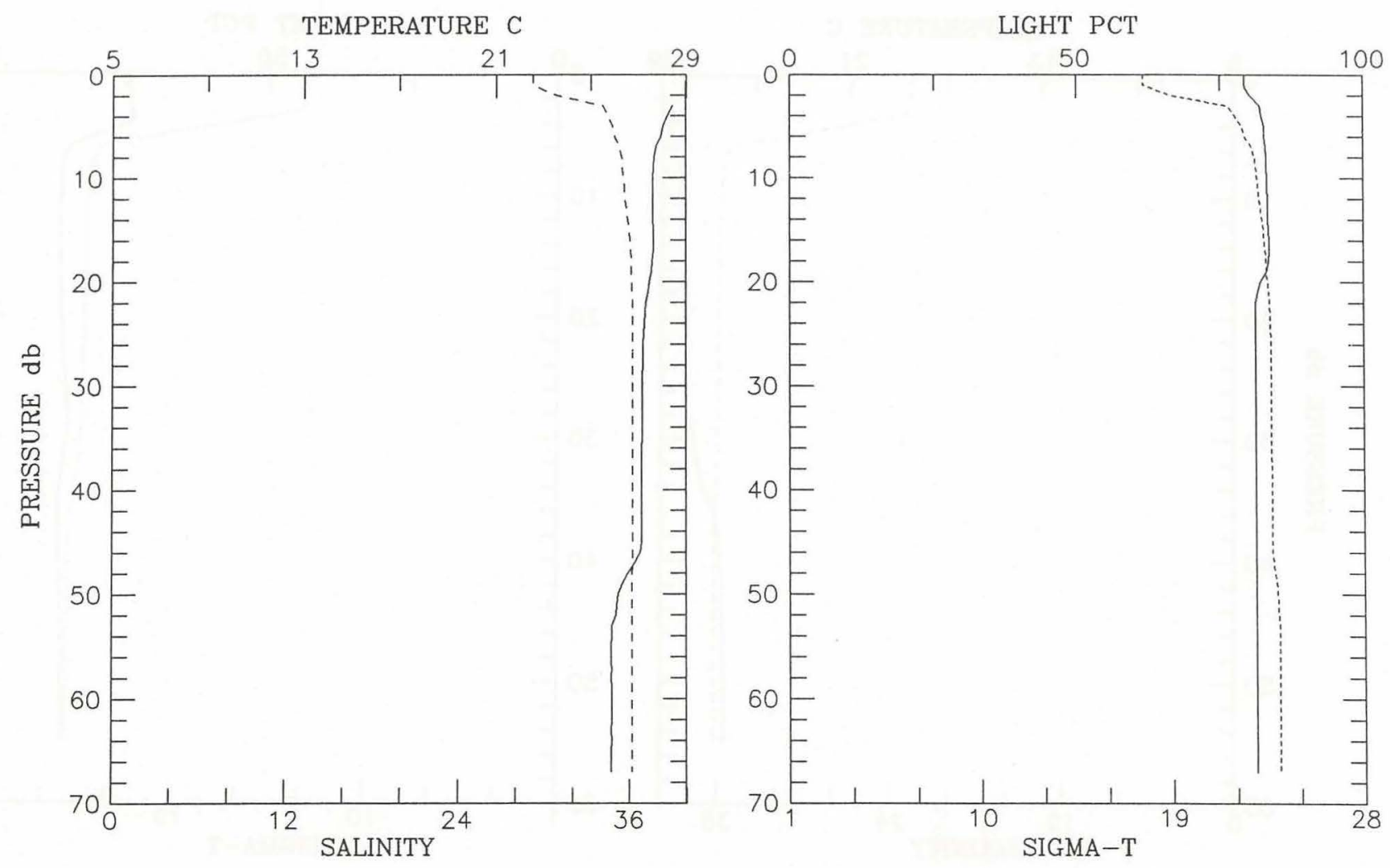


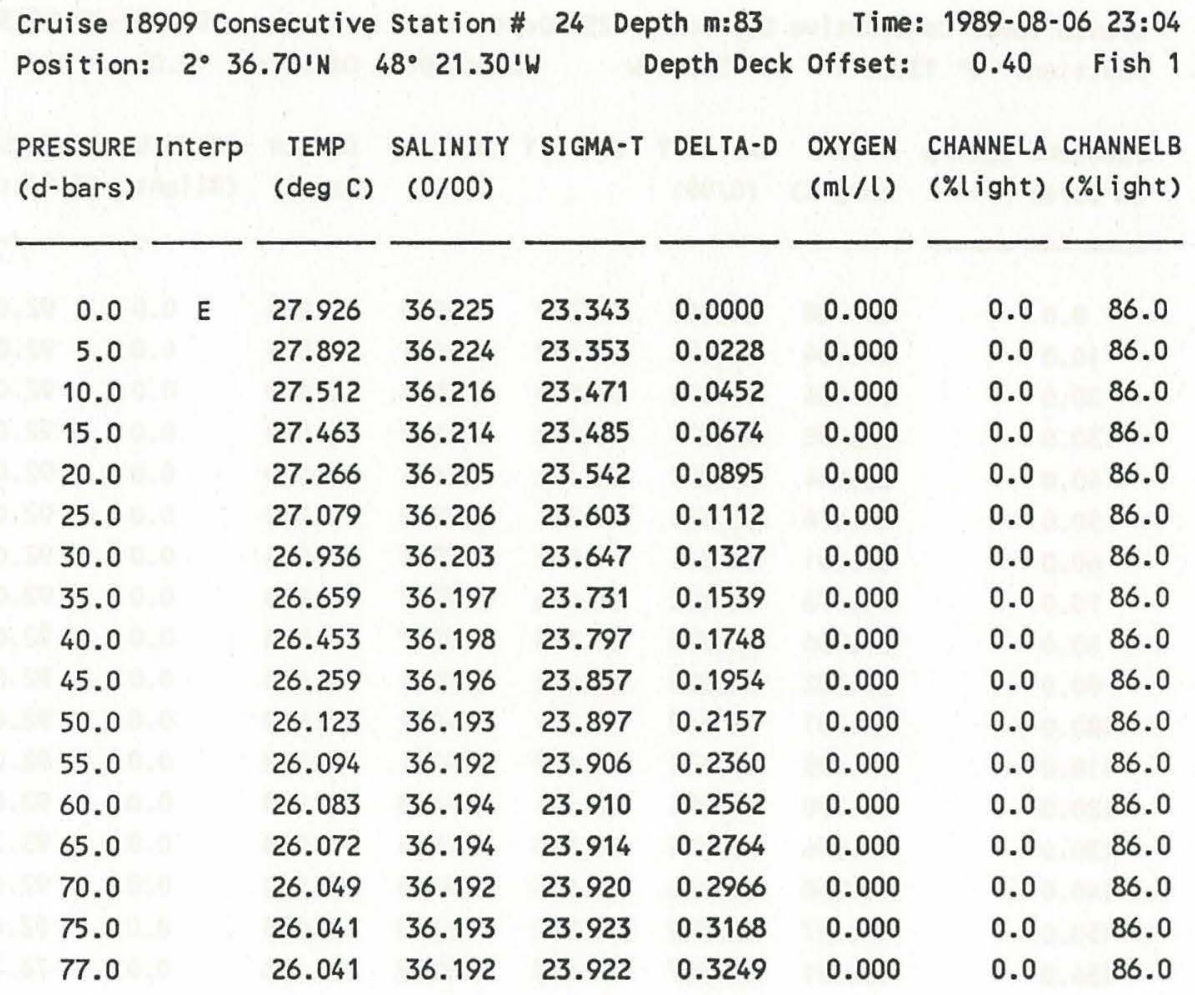

TEMPERATURE C

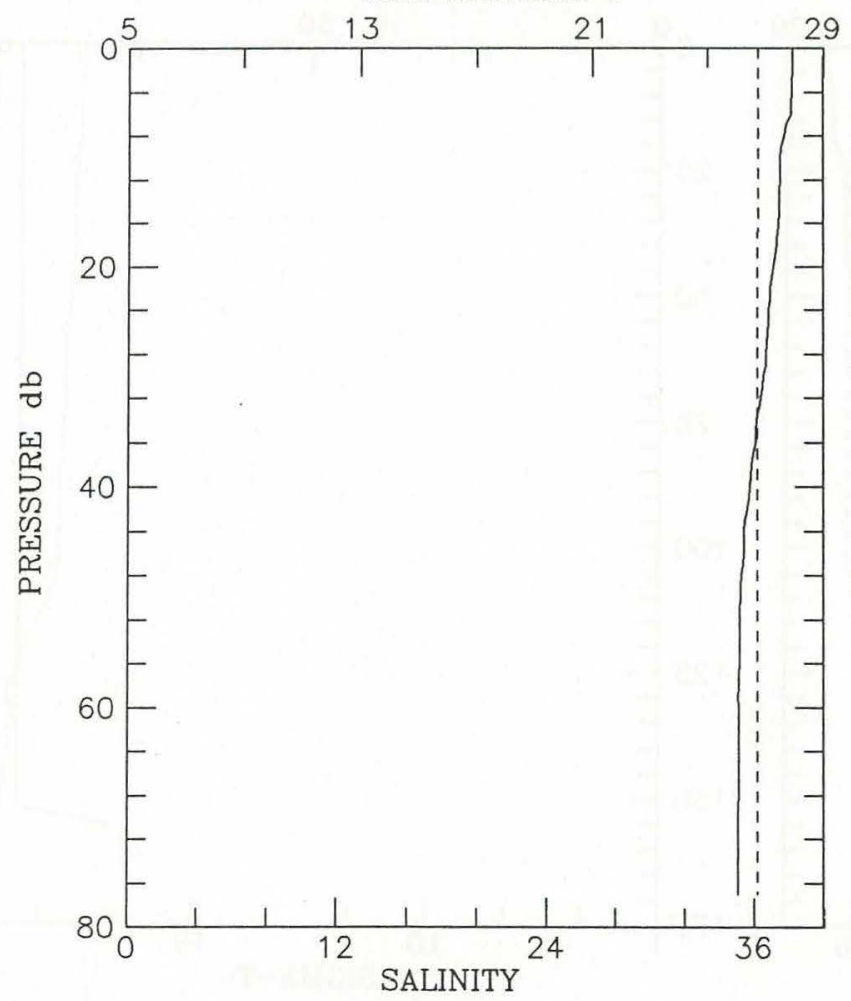

LIGHT PCT

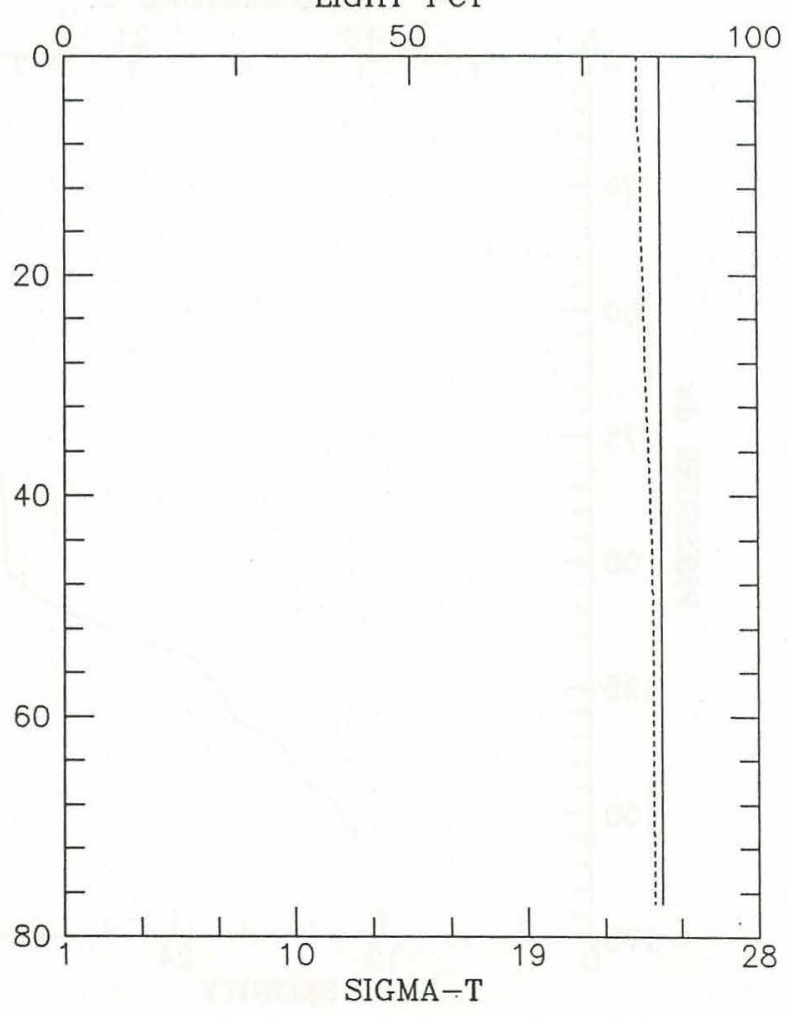




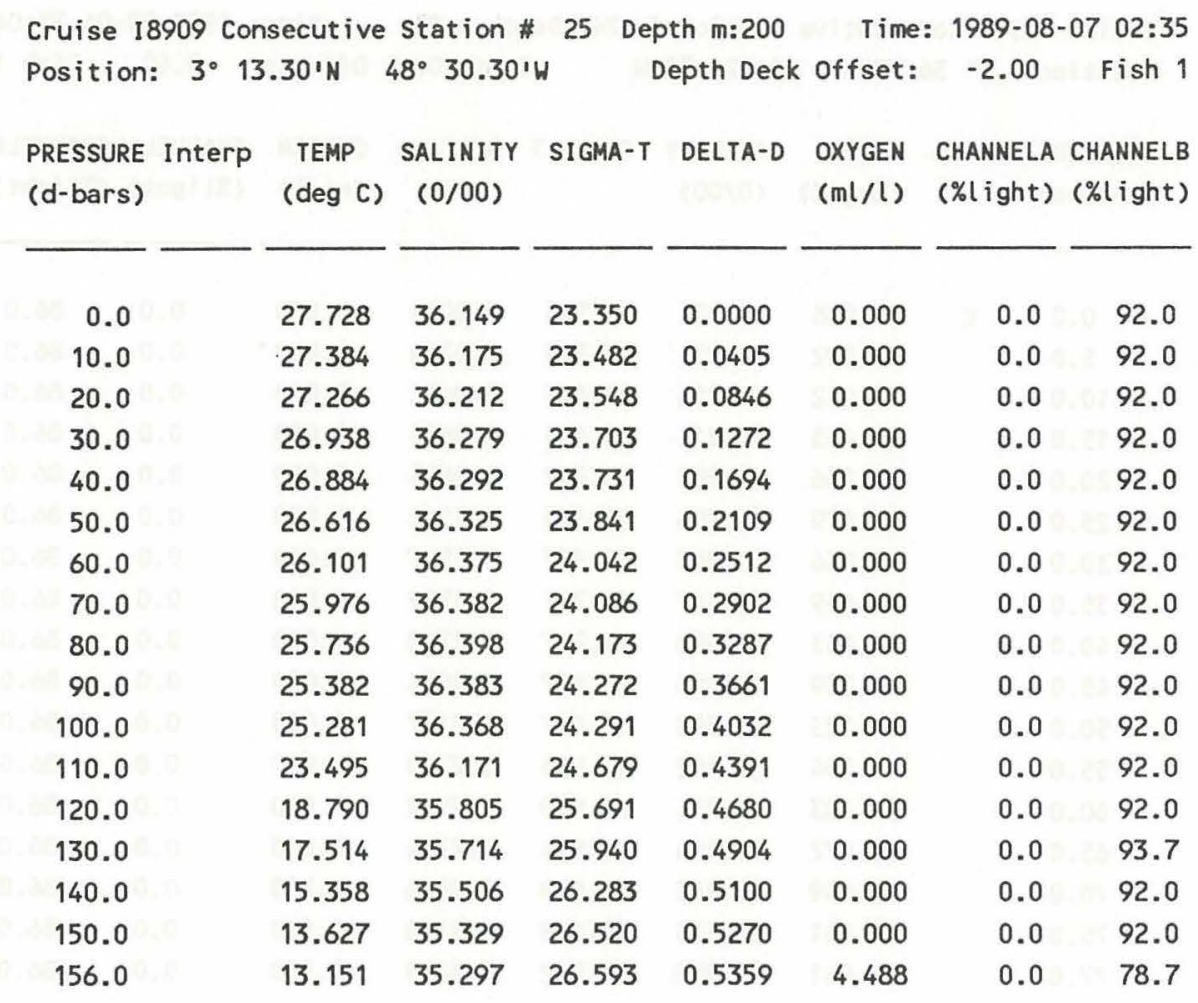
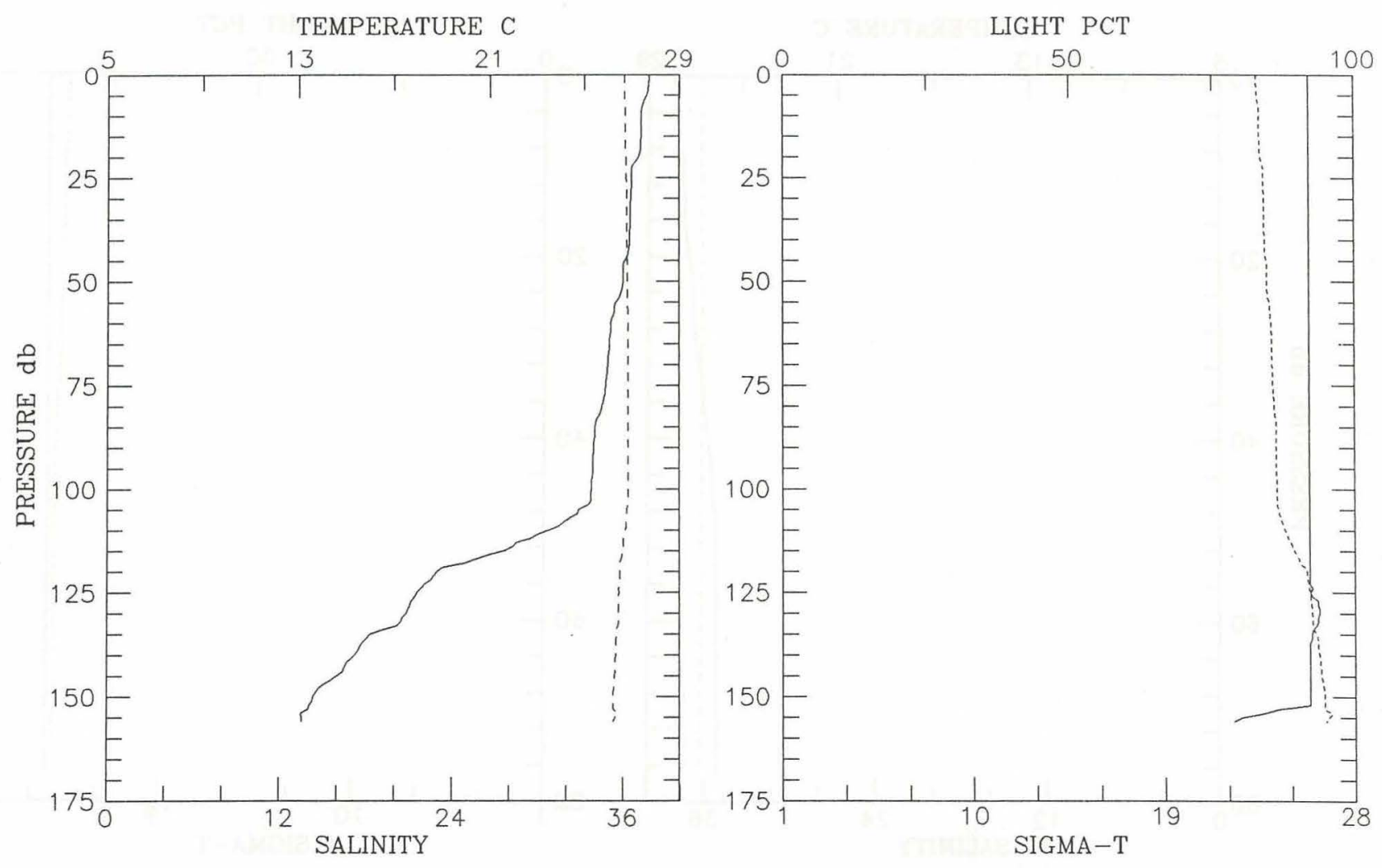


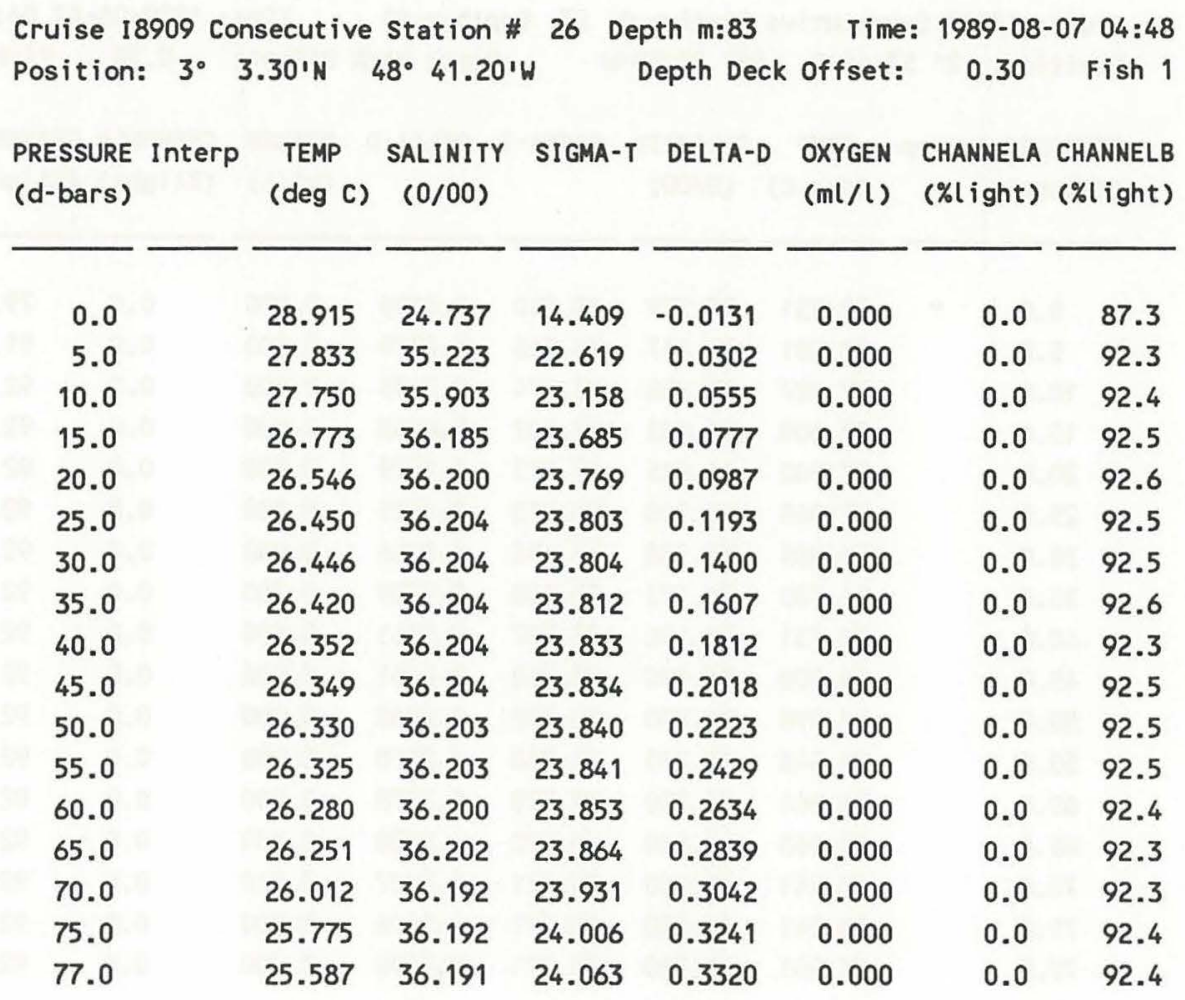
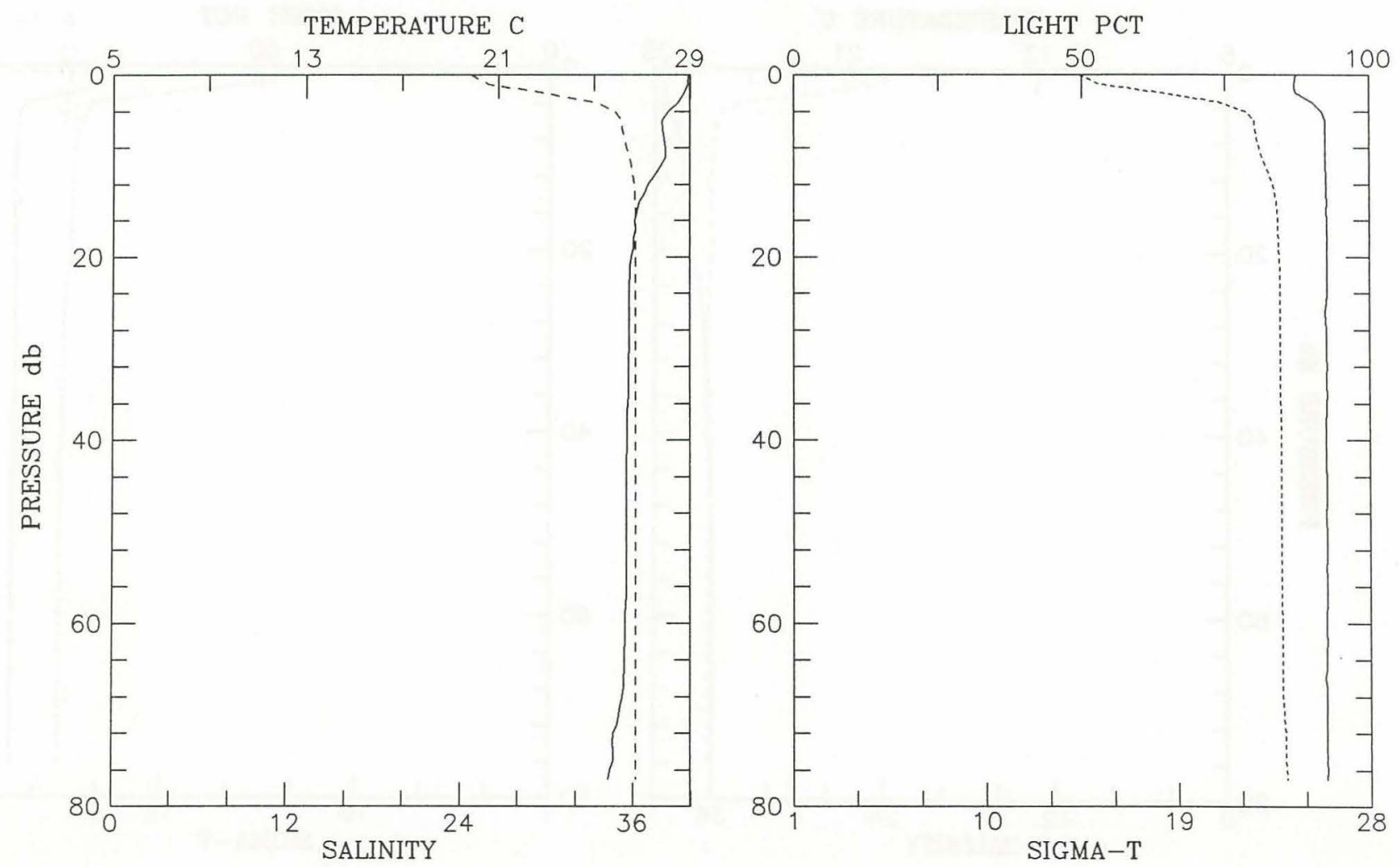
Cruise 18909 Consecutive Station \# 27 Depth m:81 Time: 1989-08-07 06:34 Position: $2^{\circ} 53.60^{\prime} \mathrm{N} \quad 48^{\circ} 48.60^{\prime} \mathrm{W} \quad$ Depth Deck offset: $0.30 \quad \mathrm{Fish} 1$

\begin{tabular}{|c|c|c|c|c|c|c|c|c|}
\hline $\begin{array}{l}\text { PRESSURE } \\
\text { (d-bars) }\end{array}$ & Interp & $\begin{array}{c}\text { TEMP } \\
(\operatorname{deg} \mathrm{C})\end{array}$ & $\begin{array}{l}\text { SALINITY } \\
(0 / 00)\end{array}$ & SIGMA-T & DELTA-D & $\begin{array}{l}\text { OXYGEN } \\
(\mathrm{ml} / \mathrm{l})\end{array}$ & $\begin{array}{l}\text { CHANNELA } \\
\text { (\%light) }\end{array}$ & $\begin{array}{l}\text { CHANNELB } \\
\text { (\%light) }\end{array}$ \\
\hline 0.0 & * & 28.551 & 23.779 & 13.810 & -0.0136 & 0.000 & 0.0 & 79.3 \\
\hline 5.0 & & 28.281 & 35.347 & 22.565 & 0.0370 & 0.000 & 0.0 & 91.8 \\
\hline 10.0 & & 27.827 & 35.958 & 23.174 & 0.0615 & 0.000 & 0.0 & 92.2 \\
\hline 15.0 & & 27.600 & 36.003 & 23.282 & 0.0848 & 0.000 & 0.0 & 92.7 \\
\hline 20.0 & & 27.502 & 36.015 & 23.323 & 0.1079 & 0.000 & 0.0 & 92.2 \\
\hline 25.0 & & 27.068 & 36.168 & 23.578 & 0.1301 & 0.000 & 0.0 & 92.4 \\
\hline 30.0 & & 26.865 & 36.188 & 23.658 & 0.1516 & 0.000 & 0.0 & 92.4 \\
\hline 35.0 & & 26.785 & 36.193 & 23.688 & 0.1729 & 0.000 & 0.0 & 92.4 \\
\hline 40.0 & & 26.731 & 36.196 & 23.707 & 0.1941 & 0.000 & 0.0 & 92.4 \\
\hline 45.0 & & 26.598 & 36.199 & 23.752 & 0.2151 & 0.000 & 0.0 & 92.7 \\
\hline 50.0 & & 26.598 & 36.200 & 23.752 & 0.2360 & 0.000 & 0.0 & 92.7 \\
\hline 55.0 & & 26.548 & 36.200 & 23.768 & 0.2570 & 0.000 & 0.0 & 92.7 \\
\hline 60.0 & & 26.544 & 36.200 & 23.770 & 0.2778 & 0.000 & 0.0 & 92.7 \\
\hline 65.0 & & 26.543 & 36.200 & 23.770 & 0.2988 & 0.000 & 0.0 & 92.7 \\
\hline 70.0 & & 26.541 & 36.200 & 23.771 & 0.3197 & 0.000 & 0.0 & 92.7 \\
\hline 75.0 & & 26.541 & 36.200 & 23.771 & 0.3406 & 0.000 & 0.0 & 92.7 \\
\hline 77.0 & & 26.541 & 36.200 & 23.771 & 0.3490 & 0.000 & 0.0 & 92.7 \\
\hline
\end{tabular}
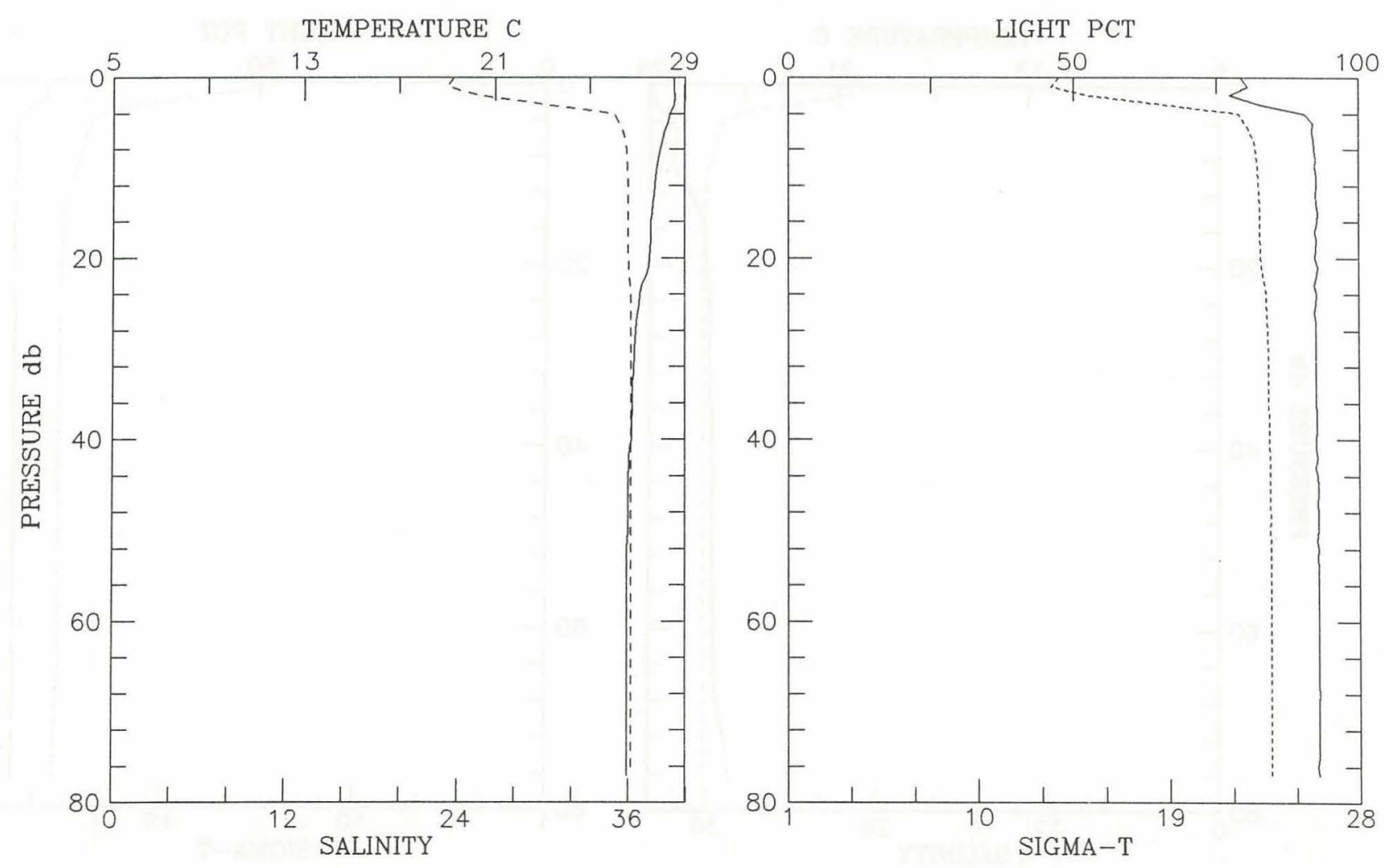


\begin{tabular}{|c|c|c|c|c|c|c|c|c|}
\hline \multicolumn{9}{|c|}{ Cruise 18909 Consecutive Station \# 28 Depth m:91 } \\
\hline Position: & $: \quad 2^{\circ} 44$ & $201 \mathrm{~N}$ & $48^{\circ} 57.10^{\prime}$ & & epth Deck & offset: & 0.30 & Fish 1 \\
\hline $\begin{array}{l}\text { PRESSURE } \\
\text { (d-bars) }\end{array}$ & Interp & $\begin{array}{c}\text { TEMP } \\
(\operatorname{deg} C)\end{array}$ & $\begin{array}{l}\text { SALINITY } \\
(0 / 00)\end{array}$ & SIGMA-T & DELTA-D & $\begin{array}{l}\text { OXYGEN } \\
(m l / l)\end{array}$ & $\begin{array}{l}\text { CHANNELA } \\
\text { (\%tight) }\end{array}$ & $\begin{array}{l}\text { CHANNELB } \\
\text { (\%light) }\end{array}$ \\
\hline 0.0 & E & 28.193 & 16.360 & 8.386 & 0.0000 & 0.000 & 0.0 & 79.1 \\
\hline 5.0 & & 28.154 & 35.542 & 22.754 & 0.0564 & 0.000 & 0.0 & 90.6 \\
\hline 10.0 & & 27.871 & 35.905 & 23.120 & 0.0812 & 0.000 & 0.0 & 90.1 \\
\hline 15.0 & & 27.469 & 36.089 & 23.389 & 0.1046 & 0.000 & 0.0 & 91.8 \\
\hline 20.0 & & 26.935 & 36.214 & 23.656 & 0.1264 & 0.000 & 0.0 & 92.7 \\
\hline 25.0 & & 26.643 & 36.215 & 23.749 & 0.1475 & 0.000 & 0.0 & 92.5 \\
\hline 30.0 & & 26.527 & 36.217 & 23.788 & 0.1684 & 0.000 & 0.0 & 92.5 \\
\hline 35.0 & & 26.475 & 36.226 & 23.811 & 0.1890 & 0.000 & 0.0 & 92.4 \\
\hline 40.0 & & 26.387 & 36.225 & 23.838 & 0.2096 & 0.000 & 0.0 & 92.3 \\
\hline 45.0 & & 26.373 & 36.226 & 23.844 & 0.2301 & 0.000 & 0.0 & 92.2 \\
\hline 50.0 & & 26.358 & 36.228 & 23.850 & 0.2506 & 0.000 & 0.0 & 92.2 \\
\hline 55.0 & & 26.367 & 36.228 & 23.847 & 0.2711 & 0.000 & 0.0 & 92.1 \\
\hline 60.0 & & 26.340 & 36.230 & 23.857 & 0.2916 & 0.000 & 0.0 & 92.0 \\
\hline 65.0 & & 26.326 & 36.230 & 23.861 & 0.3121 & 0.000 & 0.0 & 92.0 \\
\hline 70.0 & & 25.957 & 36.469 & 24.158 & 0.3324 & 0.000 & 0.0 & 91.5 \\
\hline 75.0 & & 25.956 & 36.361 & 24.076 & 0.3517 & 0.000 & 0.0 & 91.6 \\
\hline 79.0 & & 25.958 & 36.279 & 24.014 & 0.3674 & 0.000 & 0.0 & 91.5 \\
\hline
\end{tabular}
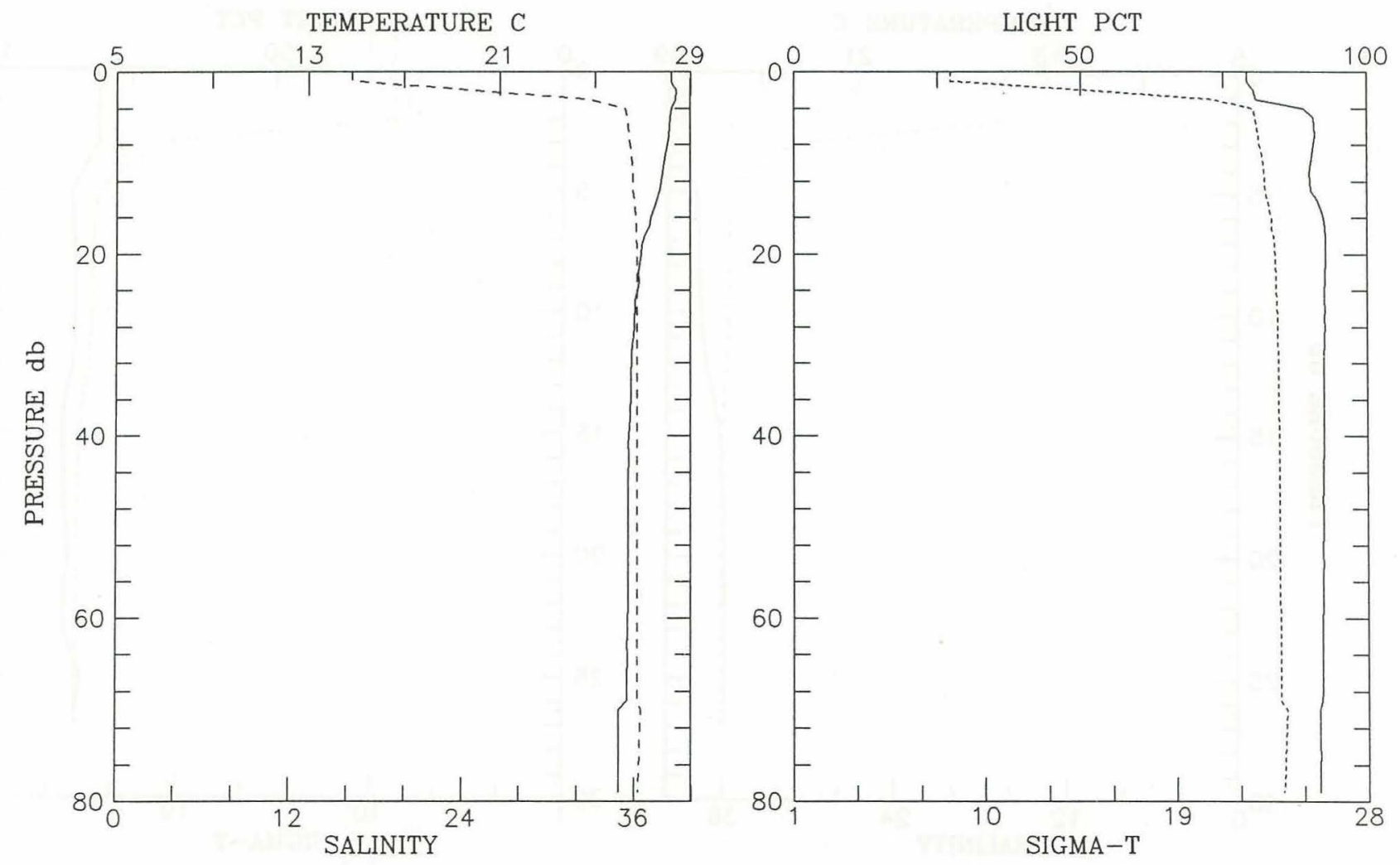


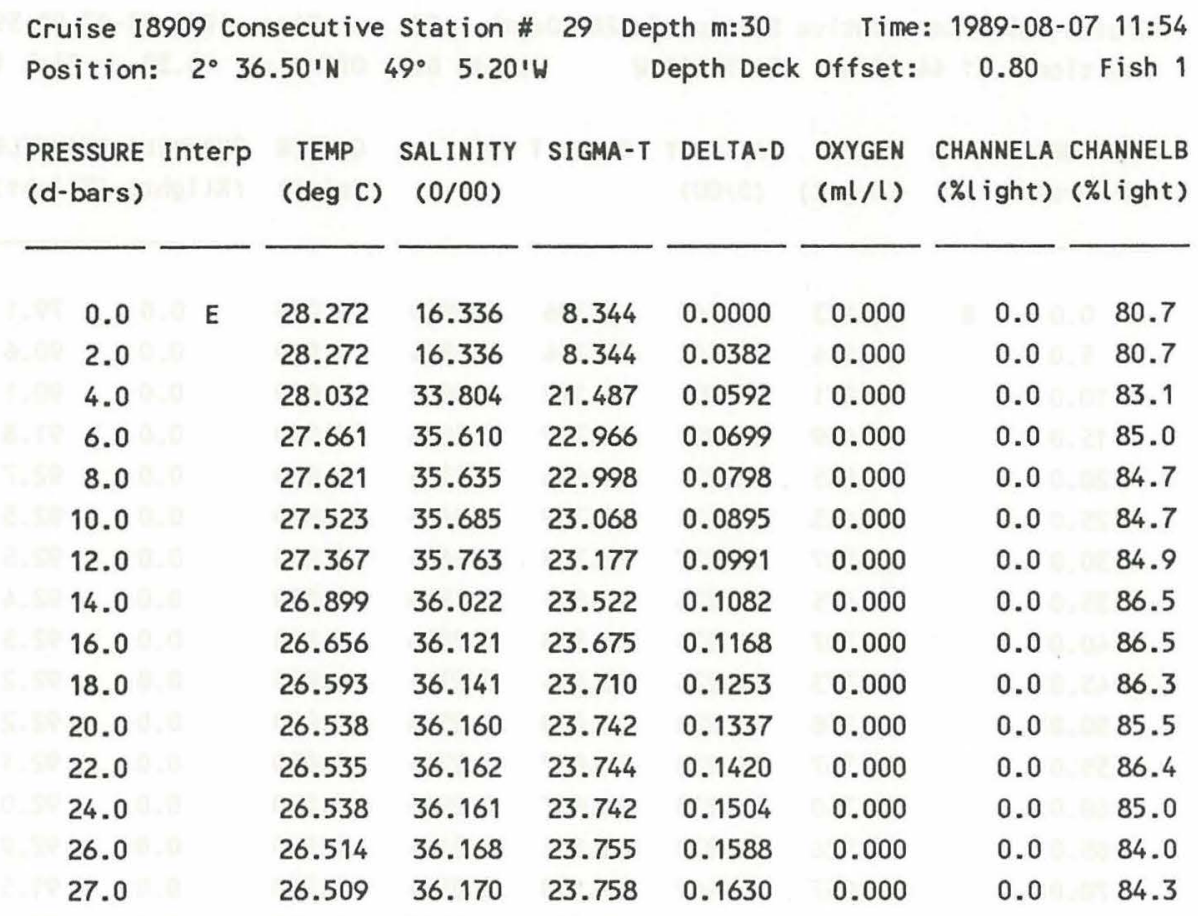
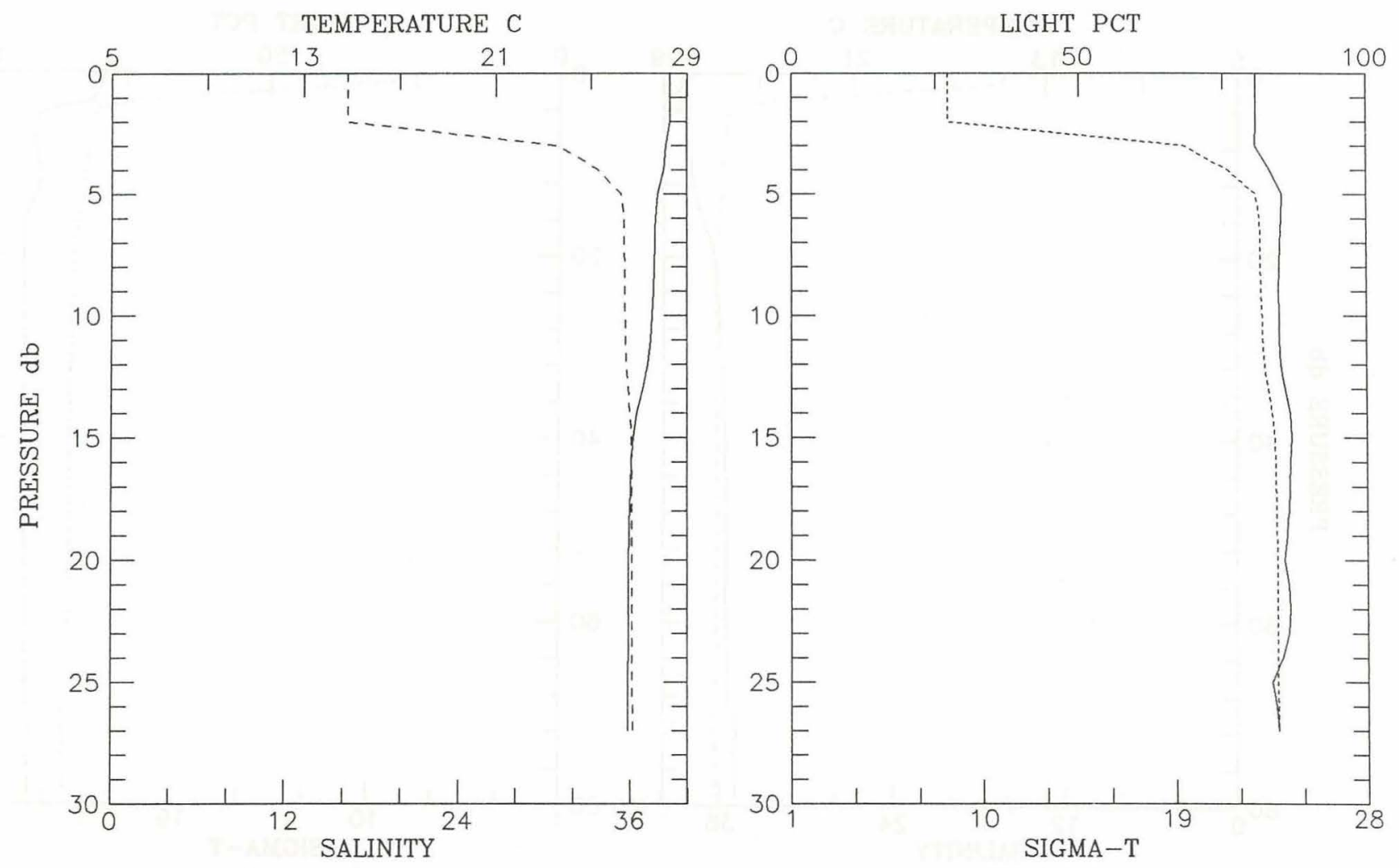


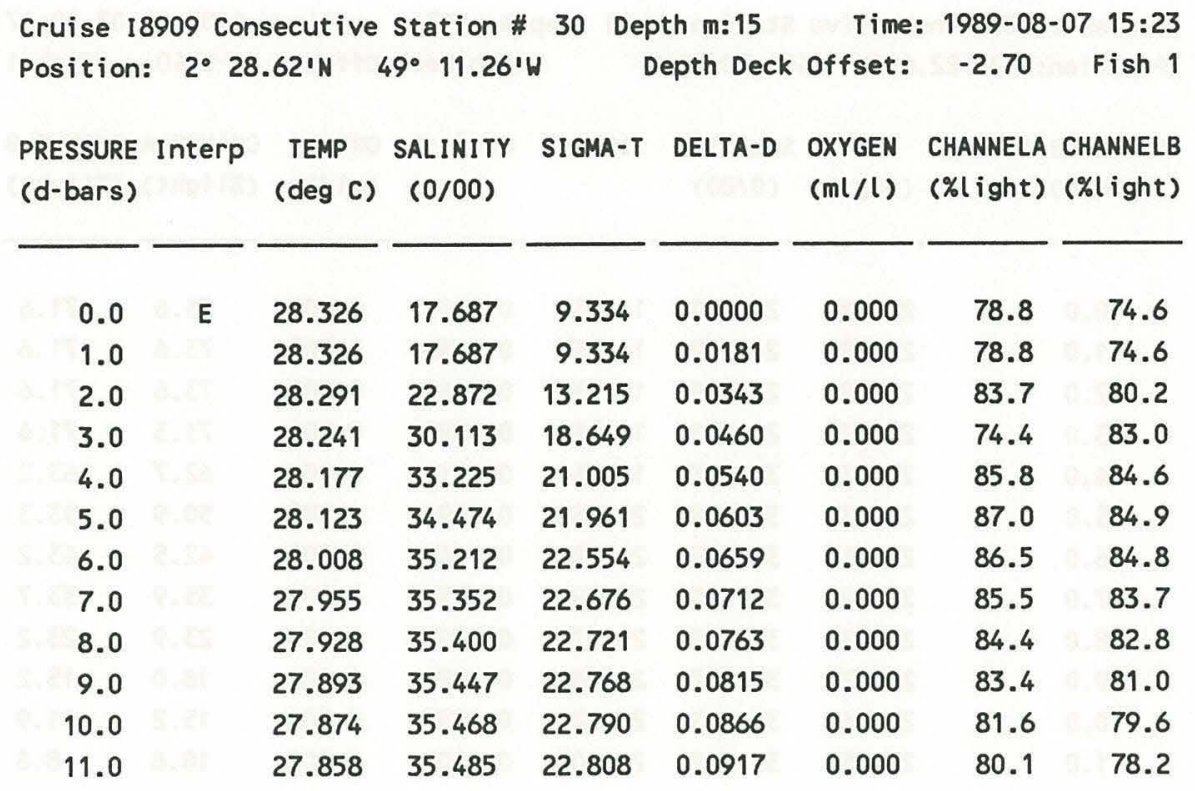
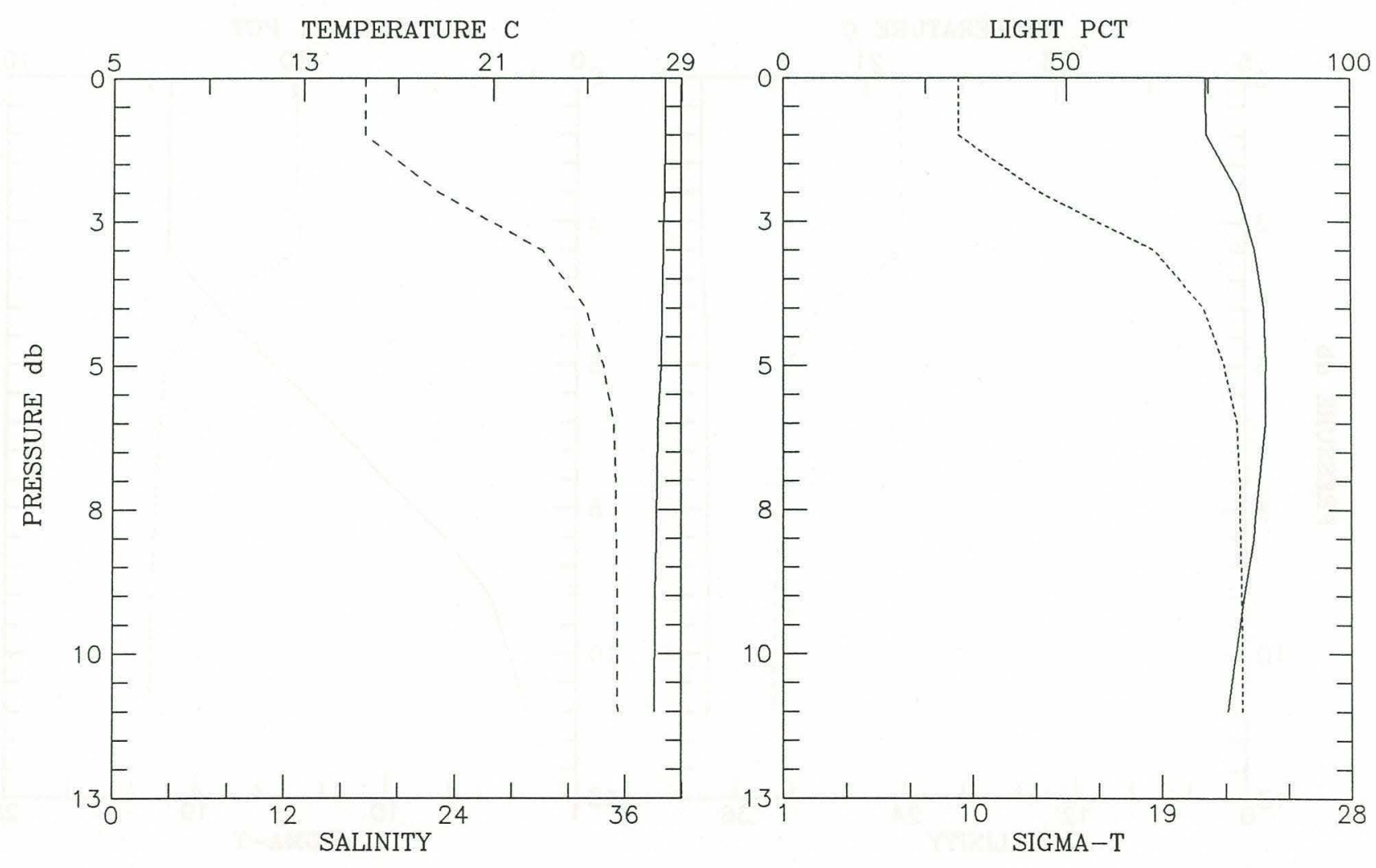
Cruise 18909 Consecutive Station \# 31 Depth m:13 Time: 1989-08-07 18:17 Position: $2^{\circ} 22.69$ ' $\mathrm{N} 49^{\circ} 20.60 \mathrm{~W}$ Wepth Deck offset: -0.40 Fish 1

\begin{tabular}{|c|c|c|c|c|c|c|c|c|}
\hline $\begin{array}{l}\text { PRESSURE } \\
\text { (d-bars) }\end{array}$ & Interp & $\begin{array}{c}\text { TEMP } \\
(\operatorname{deg} C)\end{array}$ & $\begin{array}{l}\text { SALINITY } \\
(0 / 00)\end{array}$ & SI GMA-T & DELTA-D & $\begin{array}{l}\text { OXYGEN } \\
(\mathrm{ml} / \mathrm{l})\end{array}$ & $\begin{array}{l}\text { CHANNELA } \\
\text { (\%light) }\end{array}$ & $\begin{array}{l}\text { CHANNELB } \\
\text { (\%light) }\end{array}$ \\
\hline 0.0 & E & 28.088 & 24.290 & 14.338 & 0.0000 & 0.000 & 73.6 & 71.6 \\
\hline 1.0 & $E$ & 28.088 & 24.289 & 14.337 & 0.0132 & 0.000 & 73.6 & 71.6 \\
\hline 2.0 & & 28.088 & 24.289 & 14.338 & 0.0265 & 0.000 & 73.6 & 71.6 \\
\hline 3.0 & & 28.097 & 24.001 & 14.119 & 0.0398 & 0.000 & 73.3 & 71.6 \\
\hline 4.0 & & 27.910 & 30.127 & 18.766 & 0.0510 & 0.000 & 62.7 & 63.2 \\
\hline 5.0 & & 27.833 & 32.348 & 20.458 & 0.0591 & 0.000 & 50.9 & 53.3 \\
\hline 6.0 & & 27.811 & 32.695 & 20.726 & 0.0663 & 0.000 & 42.5 & 43.2 \\
\hline 7.0 & & 27.796 & 32.923 & 20.902 & 0.0733 & 0.000 & 35.9 & 33.7 \\
\hline 8.0 & & 27.785 & 33.017 & 20.976 & 0.0802 & 0.000 & 23.9 & 23.2 \\
\hline 9.0 & & 27.771 & 33.157 & 21.086 & 0.0870 & 0.000 & 18.0 & 15.2 \\
\hline 10.0 & & 27.766 & 33.213 & 21.129 & 0.0937 & 0.000 & 15.2 & 11.9 \\
\hline 11.0 & & 27.755 & 33.311 & 21.207 & 0.1003 & 0.000 & 10.8 & 8.8 \\
\hline
\end{tabular}
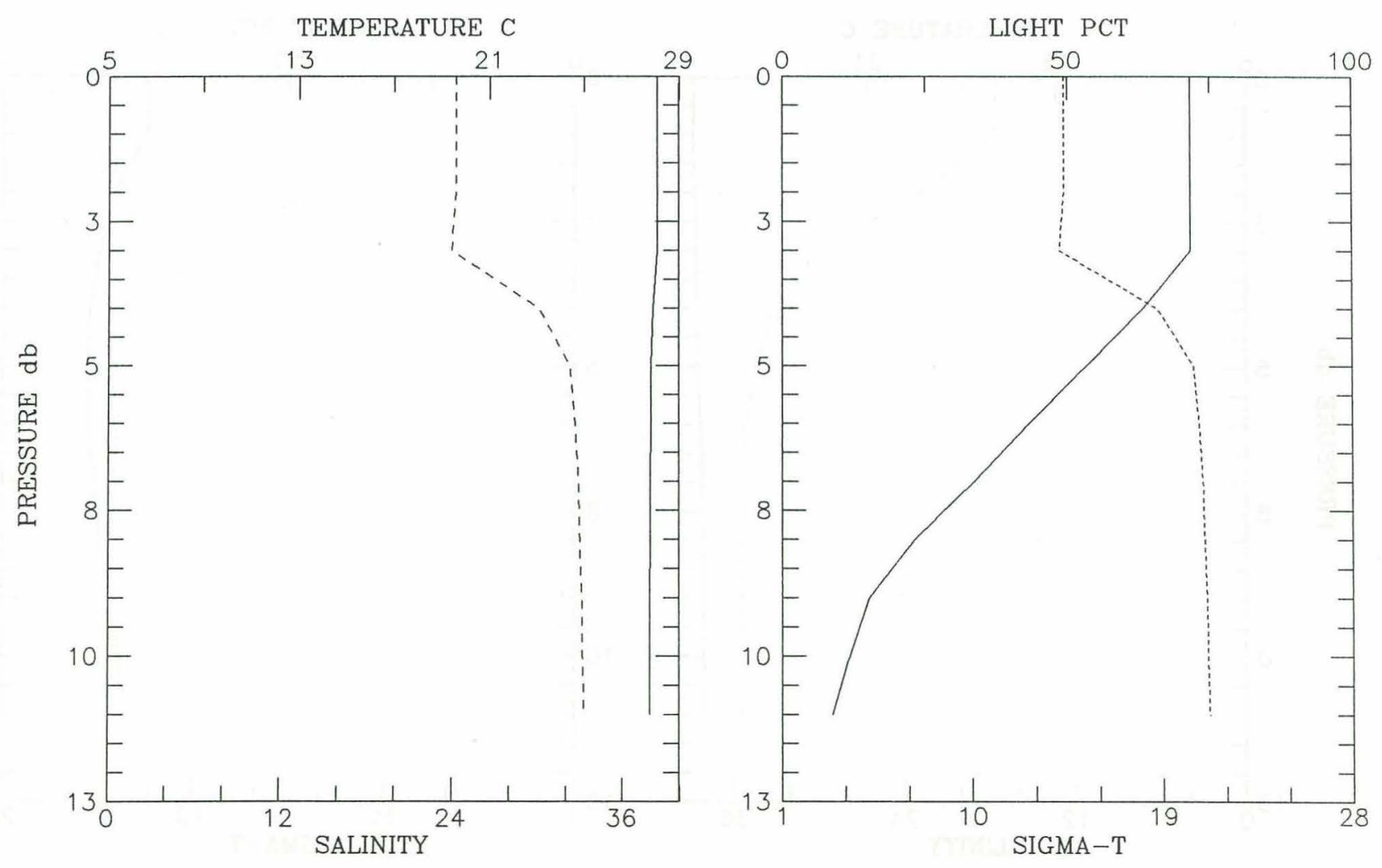


\begin{tabular}{|c|c|c|c|c|c|c|c|c|}
\hline Cruise 18 & 8909 Con & secutive & Station \# & 32 Dep & th $m: 10$ & Time: & : 1989-08- & $-0722: 50$ \\
\hline Position: & $=2^{\circ} 19$ & $.501 \mathrm{~N}$ & $50^{\circ} 9.80^{\prime}$ & & epth Deck & Offset: & 0.30 & Fish 1 \\
\hline $\begin{array}{l}\text { PRESSURE } \\
\text { (d-bars) }\end{array}$ & Interp & $\begin{array}{c}\text { TEMP } \\
(\operatorname{deg} C)\end{array}$ & $\begin{array}{c}\text { SALINITY } \\
(0 / 00)\end{array}$ & SIGMA-T & DELTA-D & $\begin{array}{l}\text { OXYGEN } \\
(\mathrm{ml} / \mathrm{l})\end{array}$ & $\begin{array}{l}\text { CHANNELA } \\
(\% \text { light })\end{array}$ & $\begin{array}{l}\text { CHANNELB } \\
\text { (\%light) }\end{array}$ \\
\hline 0.0 & E & 28.553 & 16.152 & 8.120 & 0.0000 & 0.000 & 62.3 & 61.0 \\
\hline 1.0 & & 28.553 & 16.152 & 8.120 & 0.0193 & 0.000 & 62.3 & 61.0 \\
\hline 2.0 & & 28.552 & 16.152 & 8.121 & 0.0386 & 0.000 & 62.8 & 62.8 \\
\hline 3.0 & & 28.551 & 16.144 & 8.115 & 0.0579 & 0.000 & 63.9 & 63.2 \\
\hline 4.0 & & 28.552 & 16.125 & 8.100 & 0.0773 & 0.000 & 64.8 & 63.9 \\
\hline 5.0 & & 28.551 & 16.147 & 8.117 & 0.0966 & 0.000 & 64.7 & 63.8 \\
\hline 6.0 & & 28.550 & 16.162 & 8.128 & 0.1159 & 0.000 & 64.7 & 63.8 \\
\hline 7.0 & & 28.549 & 16.177 & 8.140 & 0.1352 & 0.000 & 64.6 & 63.8 \\
\hline 8.0 & & 28.548 & 16.192 & 8.151 & 0.1545 & 0.000 & 64.6 & 63.7 \\
\hline 9.0 & & 28.548 & 16.207 & 8.163 & 0.1738 & 0.000 & 64.5 & 63.7 \\
\hline
\end{tabular}
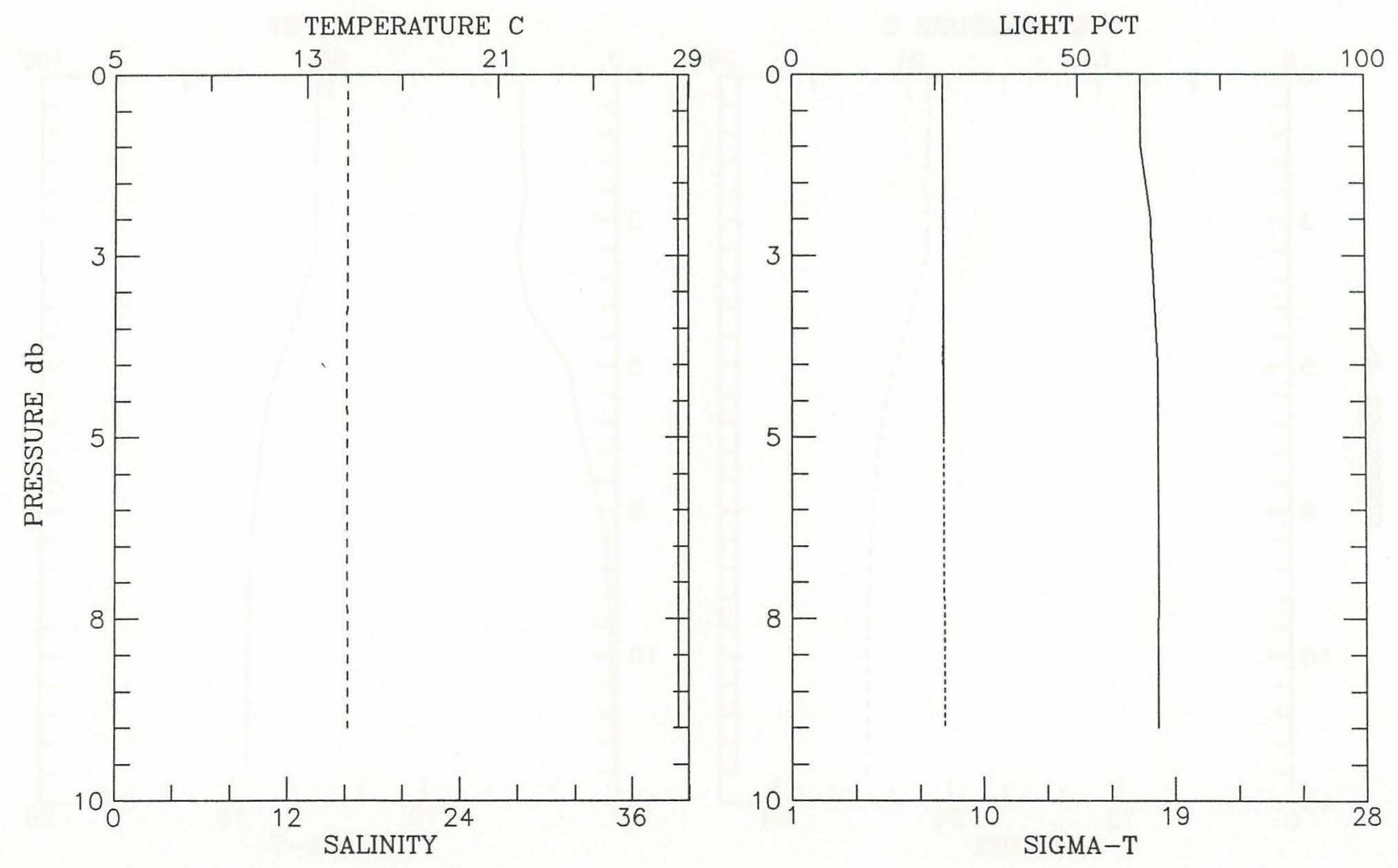
Cruise 18909 Consecutive Station \# 33 Depth m:16 Time: 1989-08-08 01:49 Position: $2^{\circ} 32.70^{\prime} \mathrm{N} \quad 49^{\circ} 53.50^{\prime} \mathrm{W} \quad$ Depth Deck offset: 0.30 Fish 1

\begin{tabular}{|c|c|c|c|c|c|c|c|c|}
\hline $\begin{array}{l}\text { PRESSURE } \\
\text { (d-bars) }\end{array}$ & Interp & $\begin{array}{c}\text { TEMP } \\
(\operatorname{deg} \mathrm{C})\end{array}$ & $\begin{array}{l}\text { SALINITY } \\
(0 / 00)\end{array}$ & SIGMA-T & DELTA-D & $\begin{array}{l}\text { OXYGEN } \\
(\mathrm{ml} / \mathrm{l})\end{array}$ & $\begin{array}{l}\text { CHANNELA } \\
\text { (\%light) }\end{array}$ & $\begin{array}{l}\text { CHANNELB } \\
\text { (\%light) }\end{array}$ \\
\hline 0.0 & $E$ & 28.190 & 25.323 & 15.079 & 0.0000 & 0.000 & 14.7 & 16.7 \\
\hline 1.0 & & 28.190 & 25.323 & 15.079 & 0.0125 & 0.000 & 14.7 & 16.7 \\
\hline 2.0 & & 28.199 & 25.446 & 15.168 & 0.0250 & 0.000 & 14.7 & 15.8 \\
\hline 3.0 & & 28.199 & 25.393 & 15.128 & 0.0374 & 0.000 & 17.0 & 17.3 \\
\hline 4.0 & & 28.229 & 26.438 & 15.900 & 0.0495 & 0.000 & 14.3 & 15.6 \\
\hline 5.0 & & 28.252 & 27.769 & 16.889 & 0.0608 & 0.000 & 8.0 & 8.5 \\
\hline 6.0 & & 28.265 & 28.692 & 17.576 & 0.0712 & 0.000 & 4.2 & 6.6 \\
\hline 7.0 & & 28.273 & 29.080 & 17.865 & 0.0812 & 0.000 & 3.8 & 4.2 \\
\hline 8.0 & & 28.274 & 29.449 & 18.141 & 0.0908 & 0.000 & 3.5 & 1.9 \\
\hline 9.0 & & 28.272 & 29.560 & 18.225 & 0.1004 & 0.000 & 2.0 & 1.3 \\
\hline 10.0 & & 28.272 & 29.625 & 18.273 & 0.1098 & 0.000 & 1.9 & 0.8 \\
\hline 11.0 & & 28.278 & 29.636 & 18.280 & 0.1192 & 0.000 & 1.0 & 0.4 \\
\hline 12.0 & & 28.279 & 29.660 & 18.297 & 0.1286 & 0.000 & 0.8 & 0.3 \\
\hline
\end{tabular}
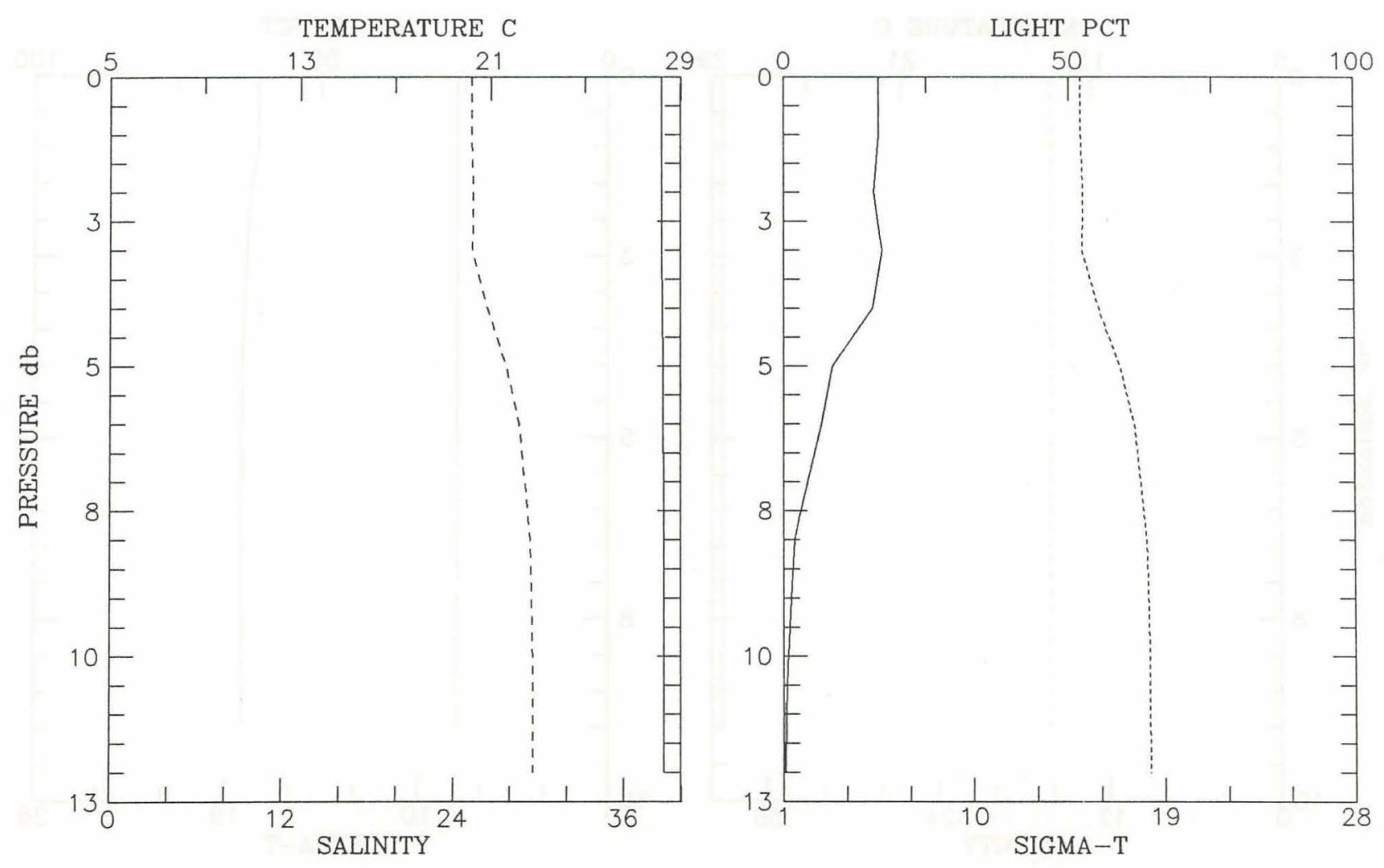


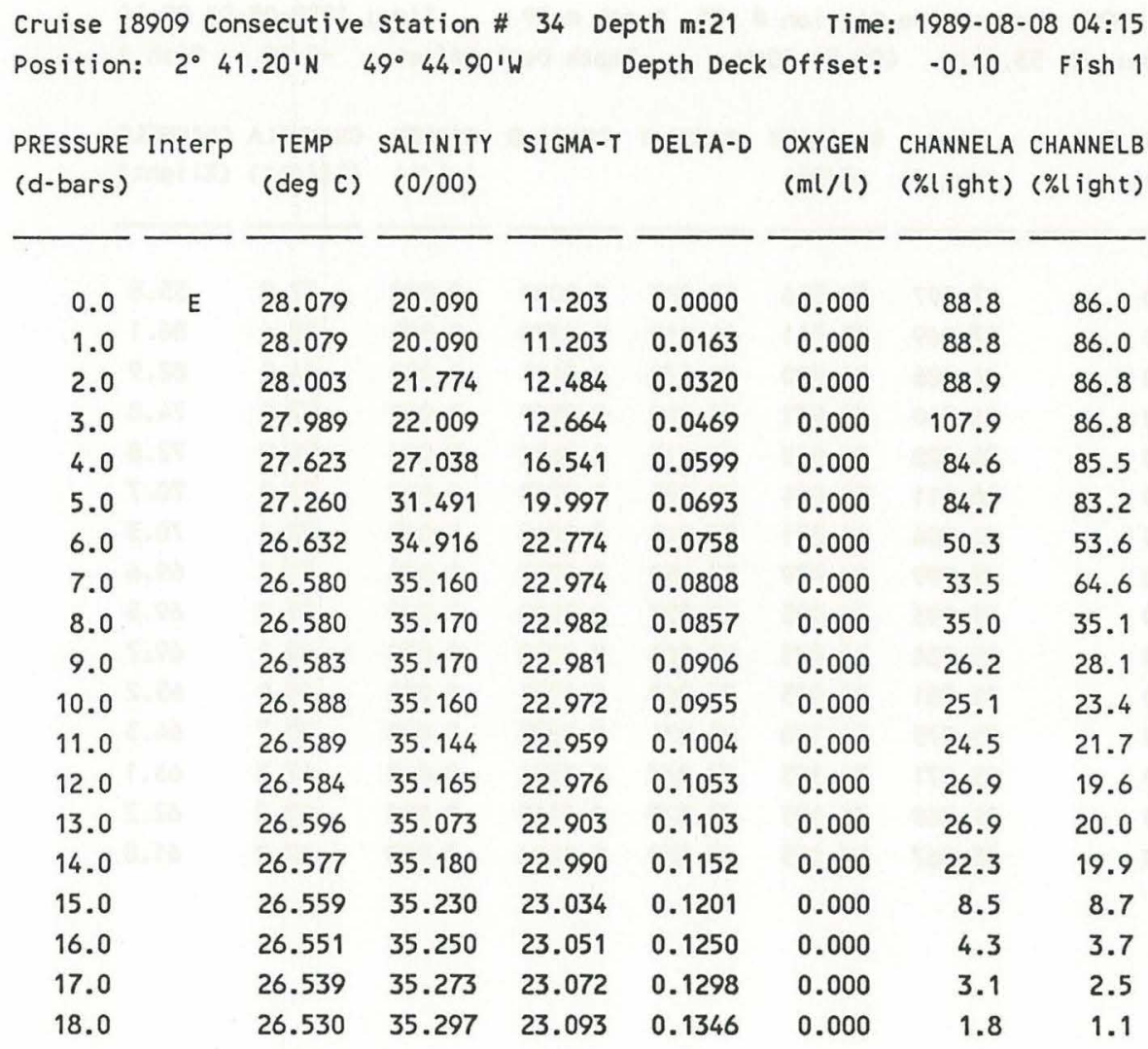
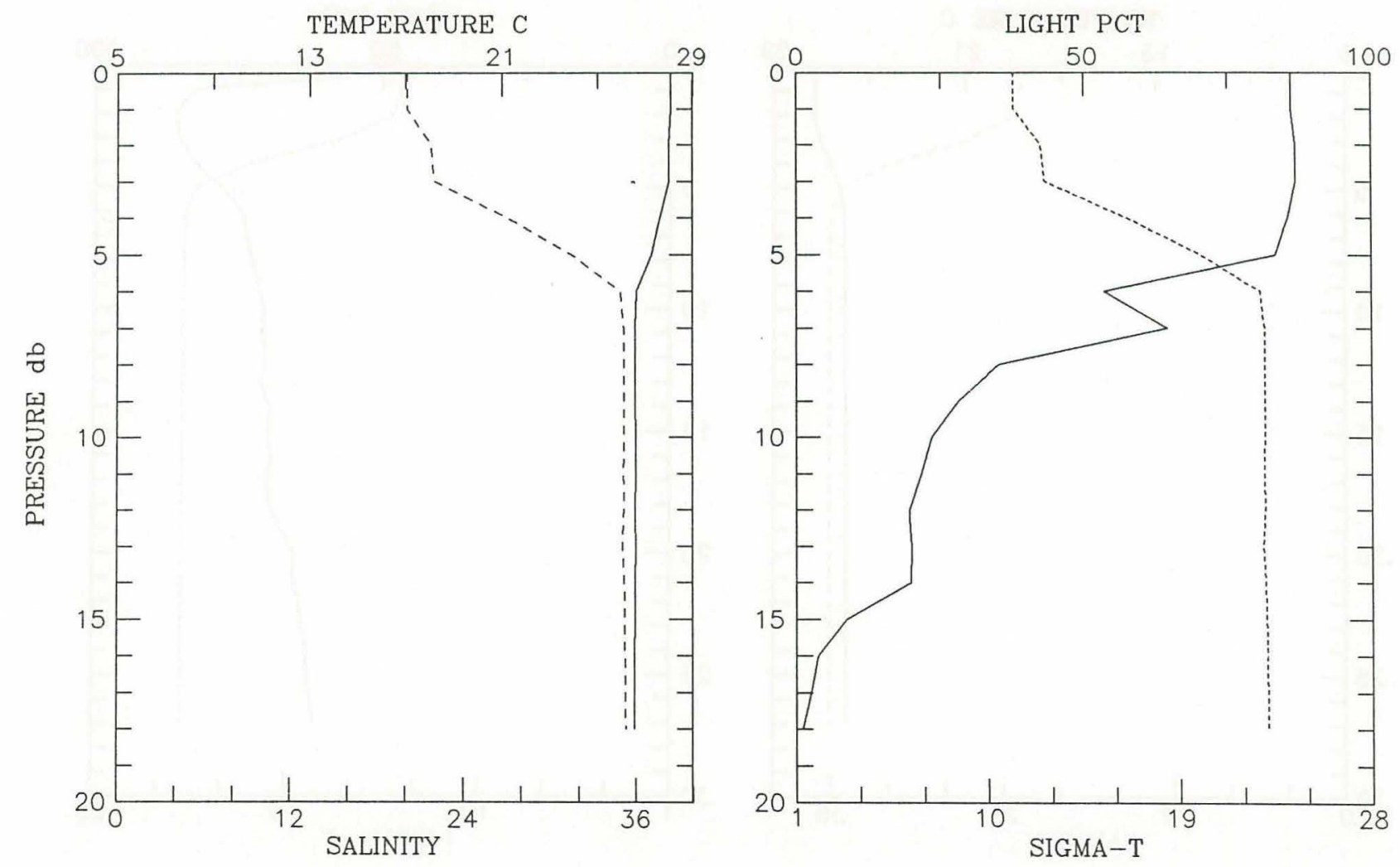


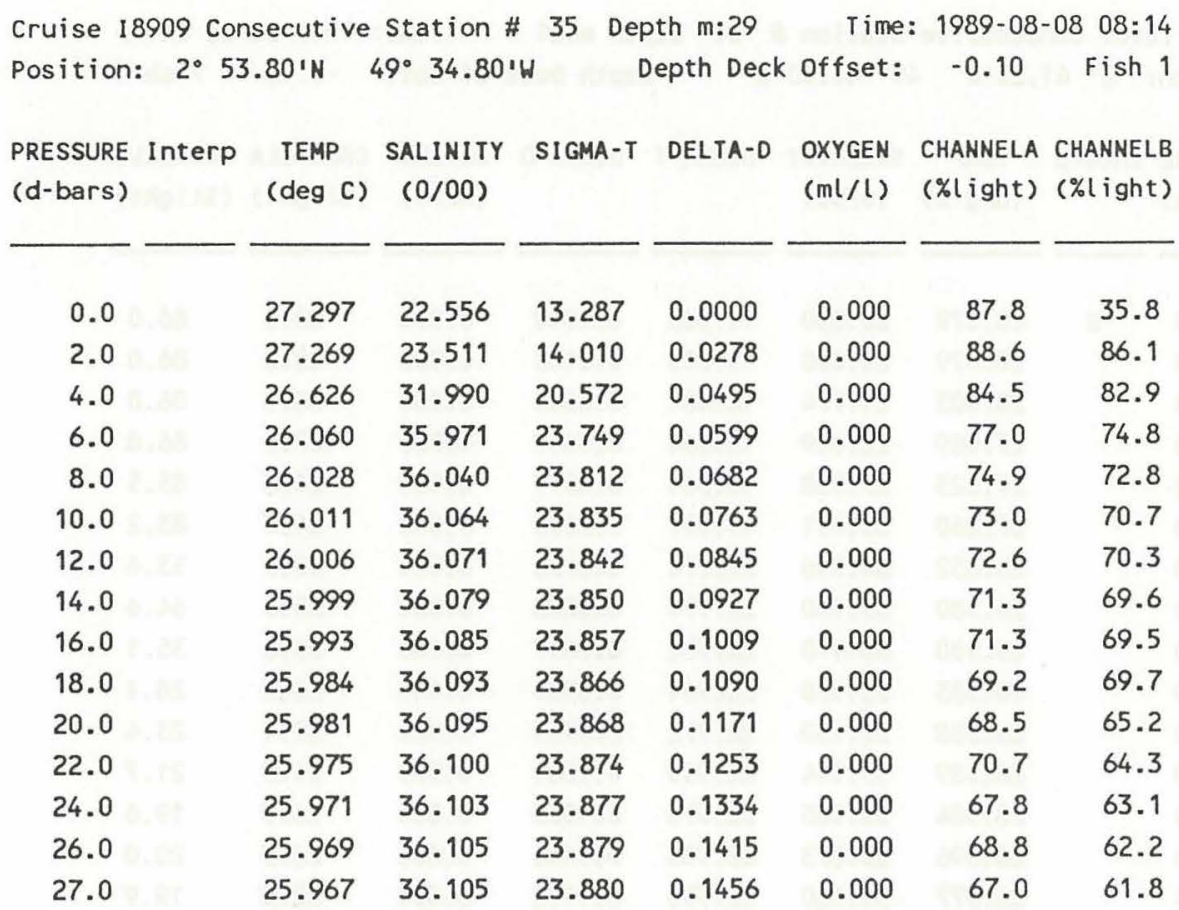
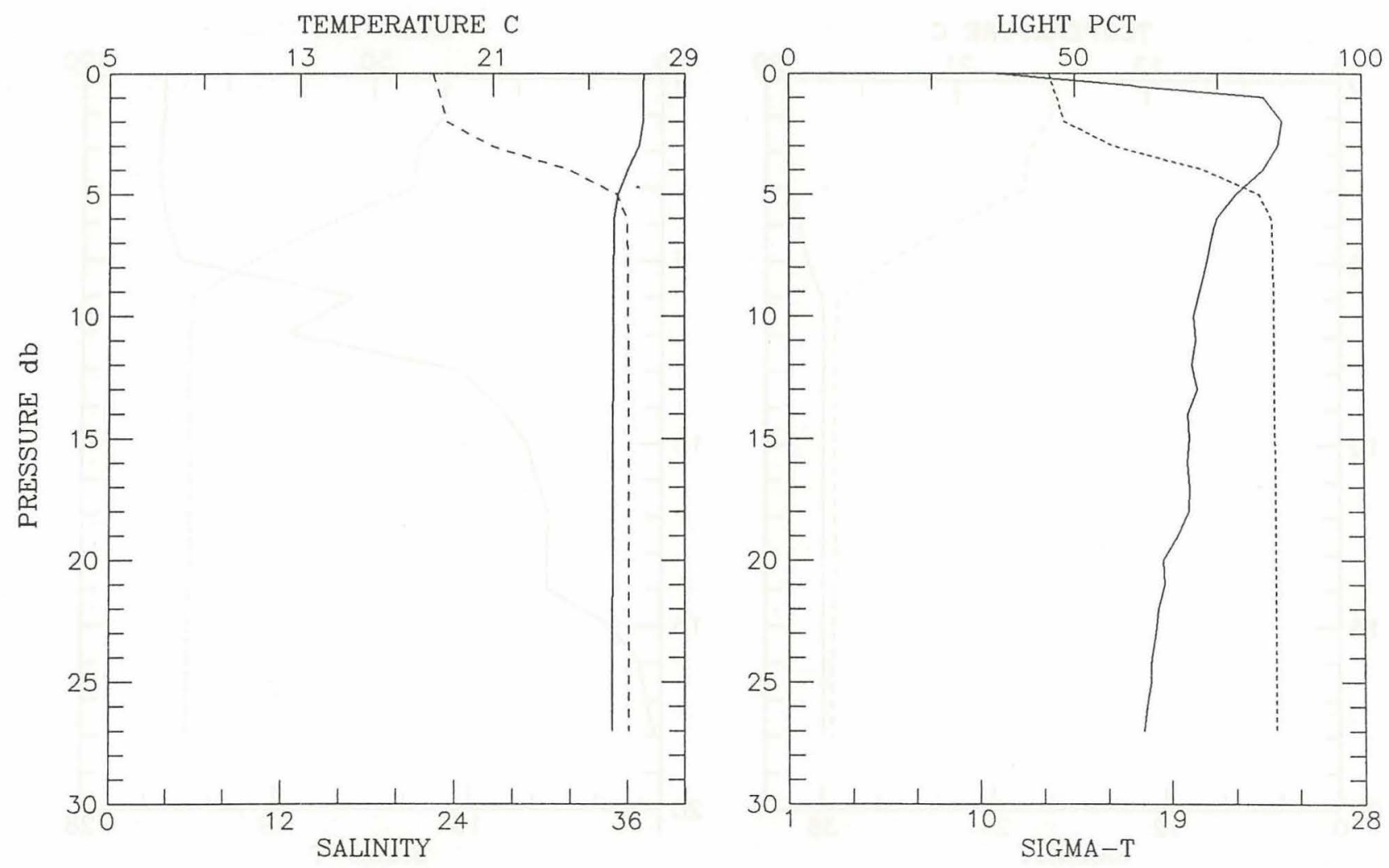


\begin{tabular}{|c|c|c|c|c|c|c|c|c|}
\hline $\begin{array}{l}\text { Cruise } 18 \\
\text { Position: }\end{array}$ & $\begin{array}{l}8909 \mathrm{C} \\
=3^{\circ}\end{array}$ & $\begin{array}{l}.201 \mathrm{~N}\end{array}$ & $\begin{array}{l}\text { Station \# } \\
49^{\circ} 17.60^{\prime}\end{array}$ & $36 D$ & $\begin{array}{l}\text { th } \pi: 81 \\
\text { epth Deck }\end{array}$ & offset: & $\begin{array}{c}1989=08 \\
0.40\end{array}$ & Fish 1 \\
\hline $\begin{array}{l}\text { PRESSURE } \\
\text { (d-bars) }\end{array}$ & Interp & $\begin{array}{c}\text { TEMP } \\
(\operatorname{deg} C)\end{array}$ & $\begin{array}{l}\text { SALINITY } \\
(0 / 00)\end{array}$ & SIGMA-T & DELTA-D & $\begin{array}{l}\text { OXYGEN } \\
(\mathrm{ml} / \mathrm{l})\end{array}$ & $\begin{array}{l}\text { CHANNELA } \\
\text { (\%light) }\end{array}$ & $\begin{array}{l}\text { CHANNELB } \\
\text { (\%light) }\end{array}$ \\
\hline 0.0 & $E$ & 28.306 & 21.862 & 12.456 & 0.0000 & 0.000 & 86.1 & 83.0 \\
\hline 5.0 & & 28.128 & 27.434 & 16.678 & 0.0701 & 0.000 & 88.7 & 85.9 \\
\hline 10.0 & & 27.636 & 35.962 & 23.239 & 0.1025 & 0.000 & 93.6 & 90.7 \\
\hline 15.0 & & 26.984 & 36.221 & 23.645 & 0.1246 & 0.000 & 95.7 & 92.8 \\
\hline 20.0 & & 26.721 & 36.219 & 23.727 & 0.1458 & 0.000 & 95.8 & 93.1 \\
\hline 25.0 & & 26.576 & 36.221 & 23.775 & 0.1667 & 0.000 & 95.8 & 93.1 \\
\hline 30.0 & & 26.497 & 36.210 & 23.792 & 0.1875 & 0.000 & 95.9 & 93.1 \\
\hline 35.0 & & 26.461 & 36.206 & 23.800 & 0.2082 & 0.000 & 95.9 & 93.1 \\
\hline 40.0 & & 26.416 & 36.204 & 23.813 & 0.2289 & 0.000 & 95.9 & 93.1 \\
\hline 45.0 & & 26.084 & 36.204 & 23.918 & 0.2494 & 0.000 & 95.8 & 93.0 \\
\hline 50.0 & & 25.698 & 36.176 & 24.017 & 0.2693 & 0.000 & 95.8 & 93.0 \\
\hline 55.0 & & 24.950 & 36.147 & 24.226 & 0.2884 & 0.000 & 95.7 & 93.0 \\
\hline 60.0 & & 24.374 & 36.133 & 24.390 & 0.3068 & 0.000 & 95.6 & 92.9 \\
\hline 65.0 & & 24.249 & 36.145 & 24.436 & 0.3246 & 0.000 & 95.4 & 92.7 \\
\hline 70.0 & & 24.103 & 36.167 & 24.497 & 0.3423 & 0.000 & 94.8 & 92.1 \\
\hline 75.0 & & 23.996 & 36.181 & 24.539 & 0.3596 & 0.000 & 94.6 & 91.8 \\
\hline 80.0 & & 23.805 & 36.174 & 24.590 & 0.3768 & 0.000 & 94.7 & 92.0 \\
\hline 81.0 & & 23.797 & 36.174 & 24.592 & 0.3802 & 0.000 & 94.7 & 92.0 \\
\hline
\end{tabular}

TEMPERATURE C

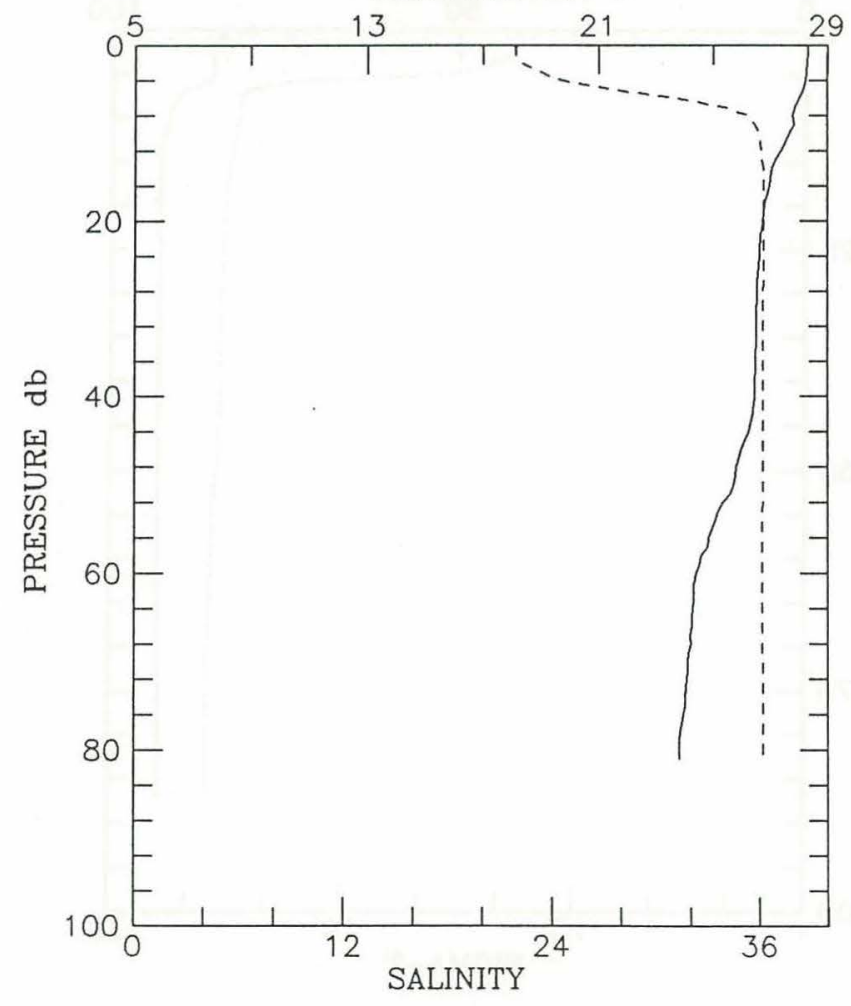

LIGHT PCT

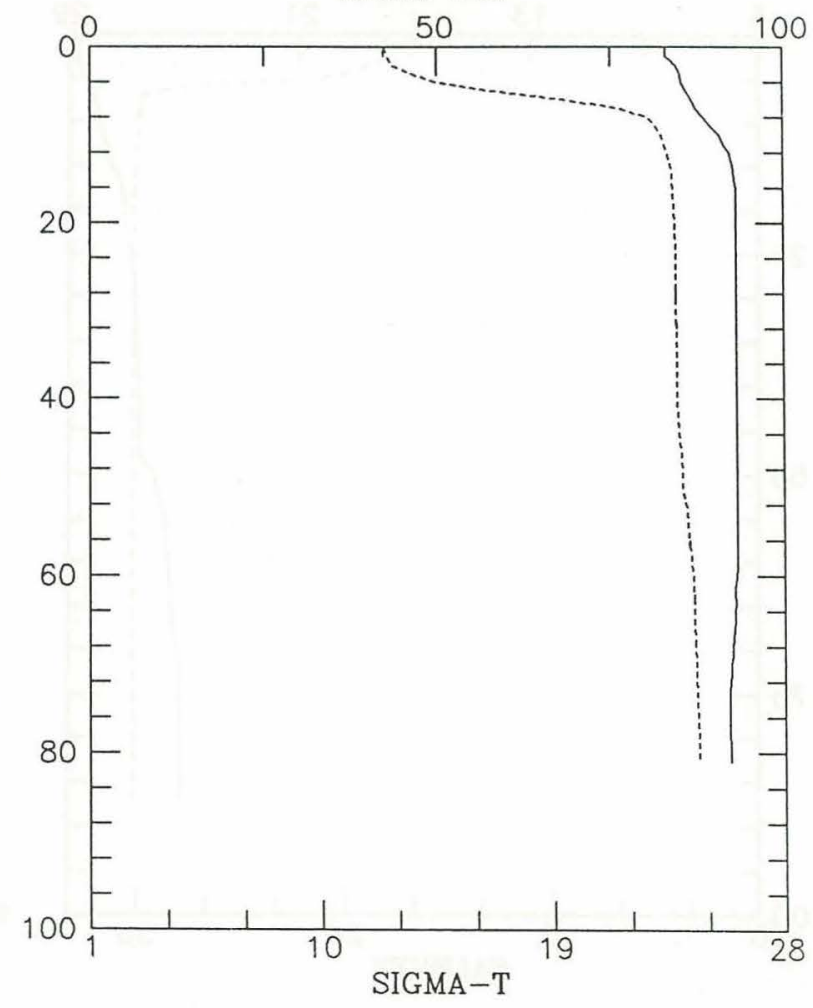


Cruise 18909 Consecutive Station \# 37 Depth m:91 Time: 1989-08-08 13:53 Position: $3^{\circ} 22.80^{\prime} \mathrm{N} \quad 49^{\circ} 7.10^{\prime} \mathrm{W} \quad$ Depth Deck offset: $0.50 \quad$ Fish 1

\begin{tabular}{|c|c|c|c|c|c|c|c|c|}
\hline $\begin{array}{l}\text { PRESSURE } \\
\text { (d-bars) }\end{array}$ & Interp & $\begin{array}{c}\text { TEMP } \\
(\operatorname{deg} C)\end{array}$ & $\begin{array}{l}\text { SALINITY } \\
(0 / 00)\end{array}$ & SI GMA-T & DELTA-D & $\begin{array}{l}\text { OXYGEN } \\
(\mathrm{ml} / \mathrm{l})\end{array}$ & $\begin{array}{l}\text { CHANNELA } \\
\text { (\%light) }\end{array}$ & $\begin{array}{l}\text { CHANNELB } \\
(\% \text { light) }\end{array}$ \\
\hline 0.0 & $E$ & 28.767 & 21.802 & 12.265 & 0.0000 & 0.000 & 87.7 & 84.5 \\
\hline 5.0 & & 28.376 & 25.320 & 15.017 & 0.0737 & 0.000 & 88.4 & 85.5 \\
\hline 10.0 & & 27.913 & 35.894 & 23.098 & 0.1036 & 0.000 & 94.3 & 91.7 \\
\hline 15.0 & & 27.529 & 36.036 & 23.330 & 0.1270 & 0.000 & 95.5 & 92.8 \\
\hline 20.0 & & 27.017 & 36.154 & 23.584 & 0.1490 & 0.000 & 95.5 & 93.0 \\
\hline 25.0 & & 26.850 & 36.184 & 23.660 & 0.1705 & 0.000 & 95.6 & 92.9 \\
\hline 30.0 & & 26.690 & 36.204 & 23.726 & 0.1916 & 0.000 & 95.5 & 92.9 \\
\hline 35.0 & & 26.618 & 36.209 & 23.753 & 0.2126 & 0.000 & 95.6 & 93.0 \\
\hline 40.0 & & 26.550 & 36.213 & 23.778 & 0.2335 & 0.000 & 95.6 & 93.0 \\
\hline 45.0 & & 26.500 & 36.219 & 23.798 & 0.2542 & 0.000 & 95.7 & 93.0 \\
\hline 50.0 & & 26.061 & 36.207 & 23.927 & 0.2748 & 0.000 & 95.6 & 93.0 \\
\hline 55.0 & & 25.594 & 36.215 & 24.079 & 0.2946 & 0.000 & 95.6 & 93.0 \\
\hline 60.0 & & 25.528 & 36.218 & 24.102 & 0.3140 & 0.000 & 95.6 & 93.0 \\
\hline 65.0 & & 25.421 & 36.211 & 24.130 & 0.3332 & 0.000 & 95.6 & 93.0 \\
\hline 70.0 & & 25.163 & 36.215 & 24.212 & 0.3522 & 0.000 & 95.5 & 92.9 \\
\hline 75.0 & & 25.115 & 36.205 & 24.219 & 0.3710 & 0.000 & 95.5 & 92.9 \\
\hline 80.0 & & 25.078 & 36.205 & 24.231 & 0.3898 & 0.000 & 95.5 & 92.9 \\
\hline 85.0 & & 25.045 & 36.203 & 24.239 & 0.4085 & 0.000 & 95.3 & 92.8 \\
\hline 87.0 & & 25.050 & 36.203 & 24.238 & 0.4160 & 0.000 & 95.4 & 92.8 \\
\hline
\end{tabular}
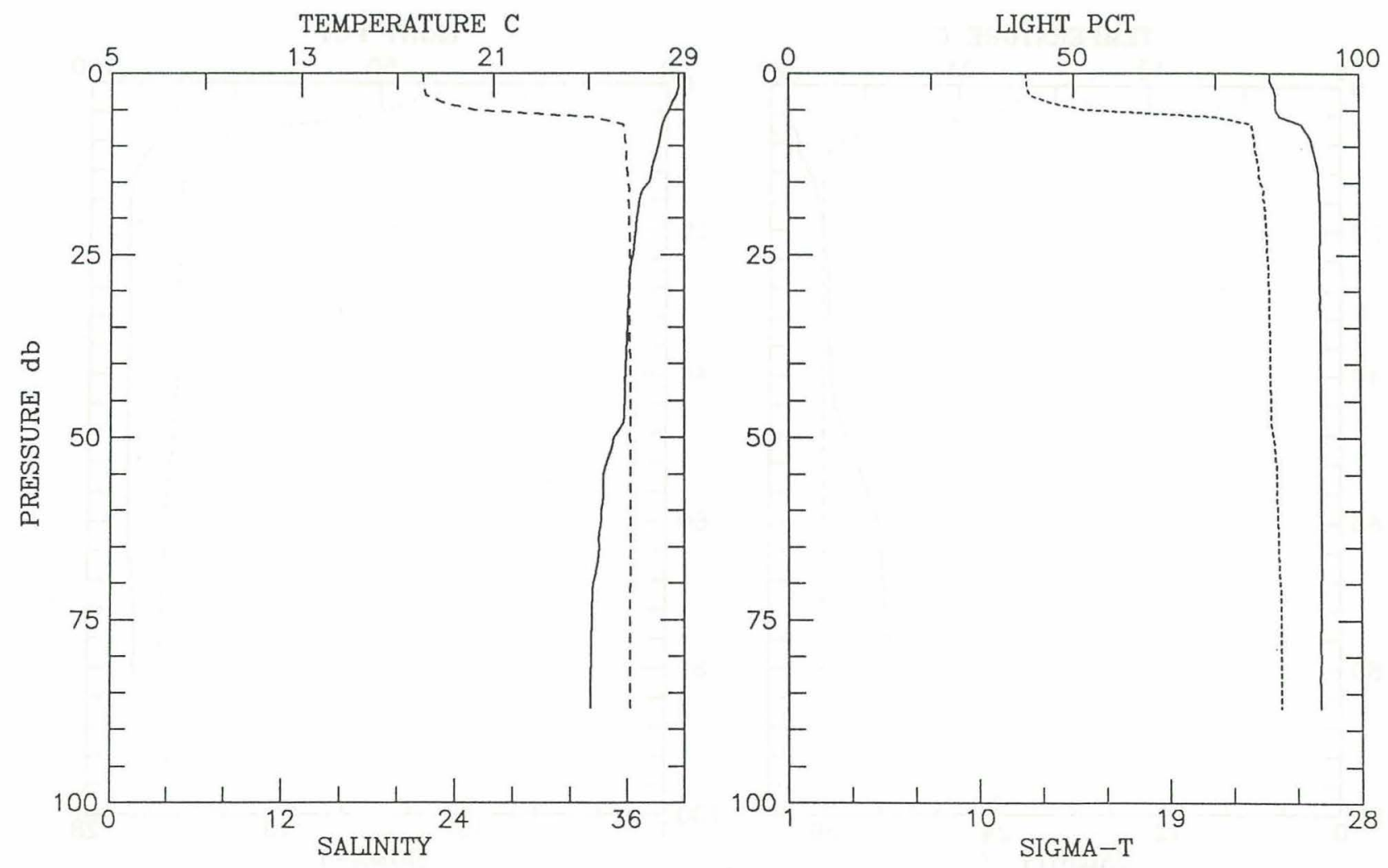


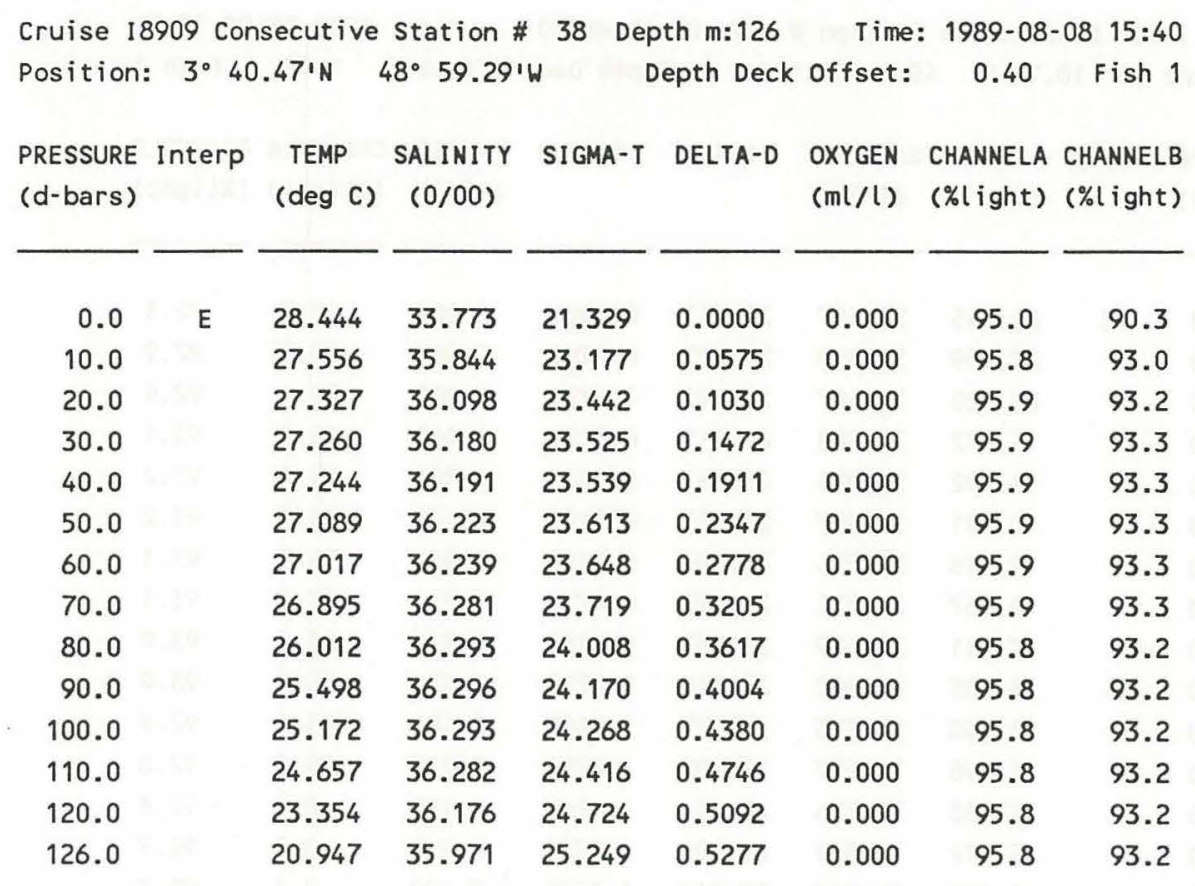
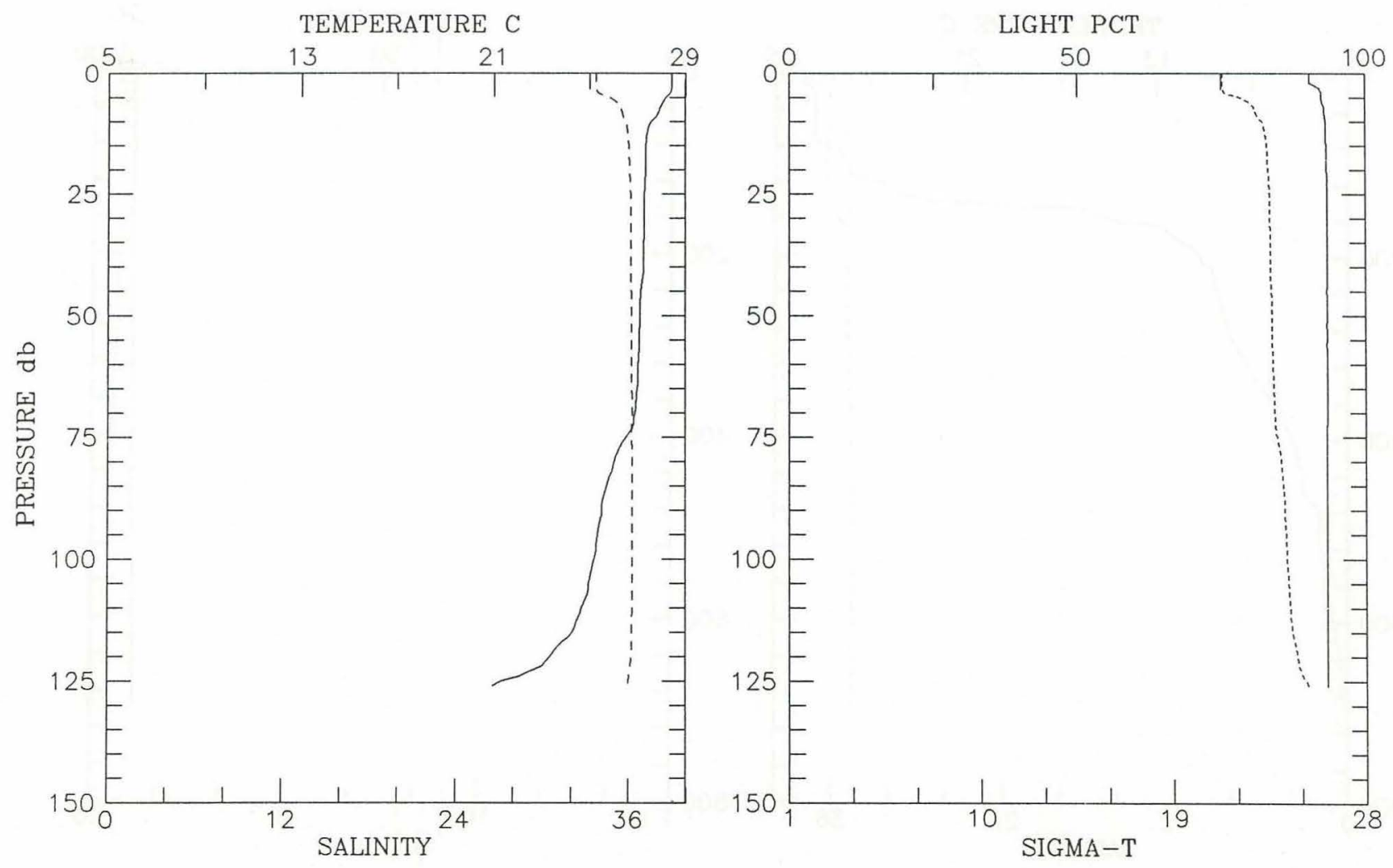


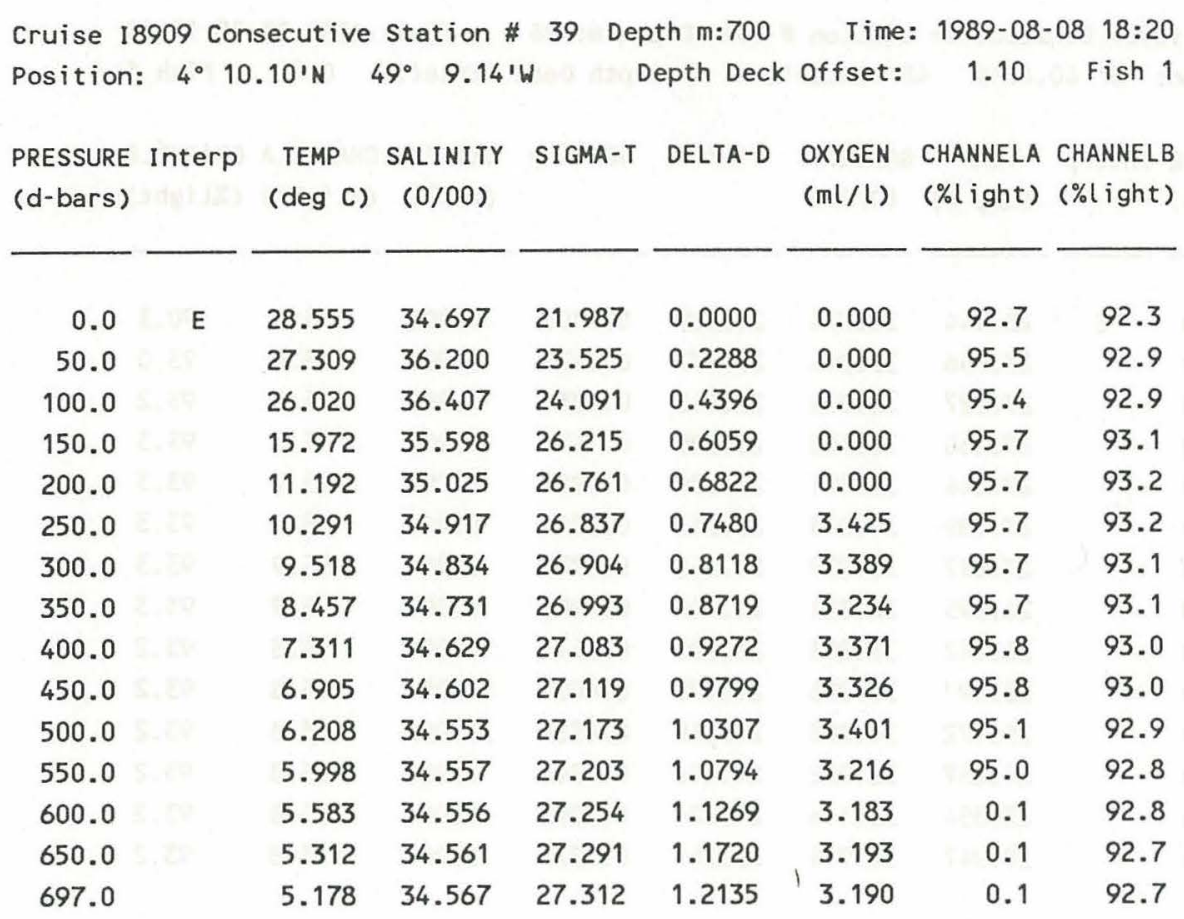
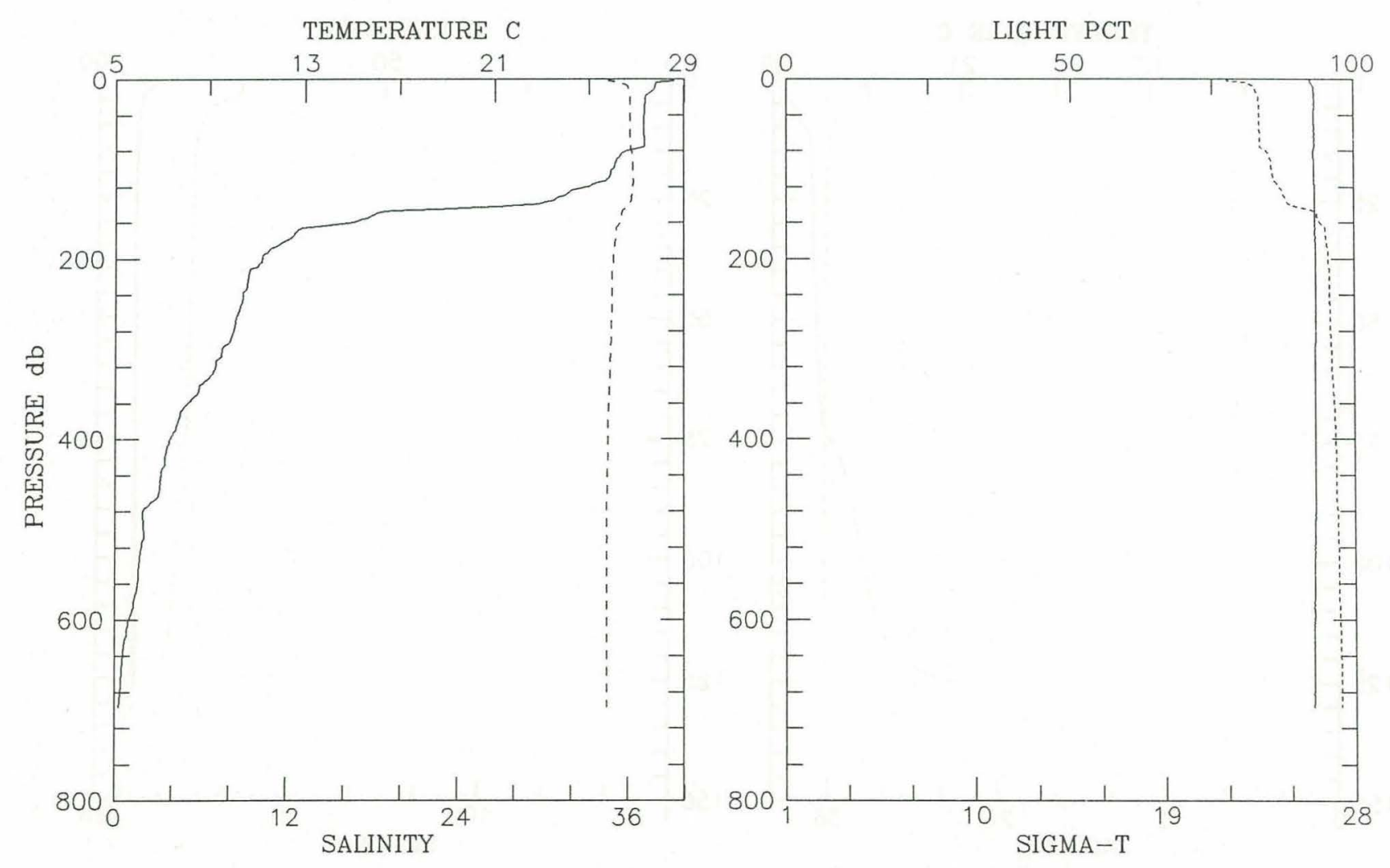


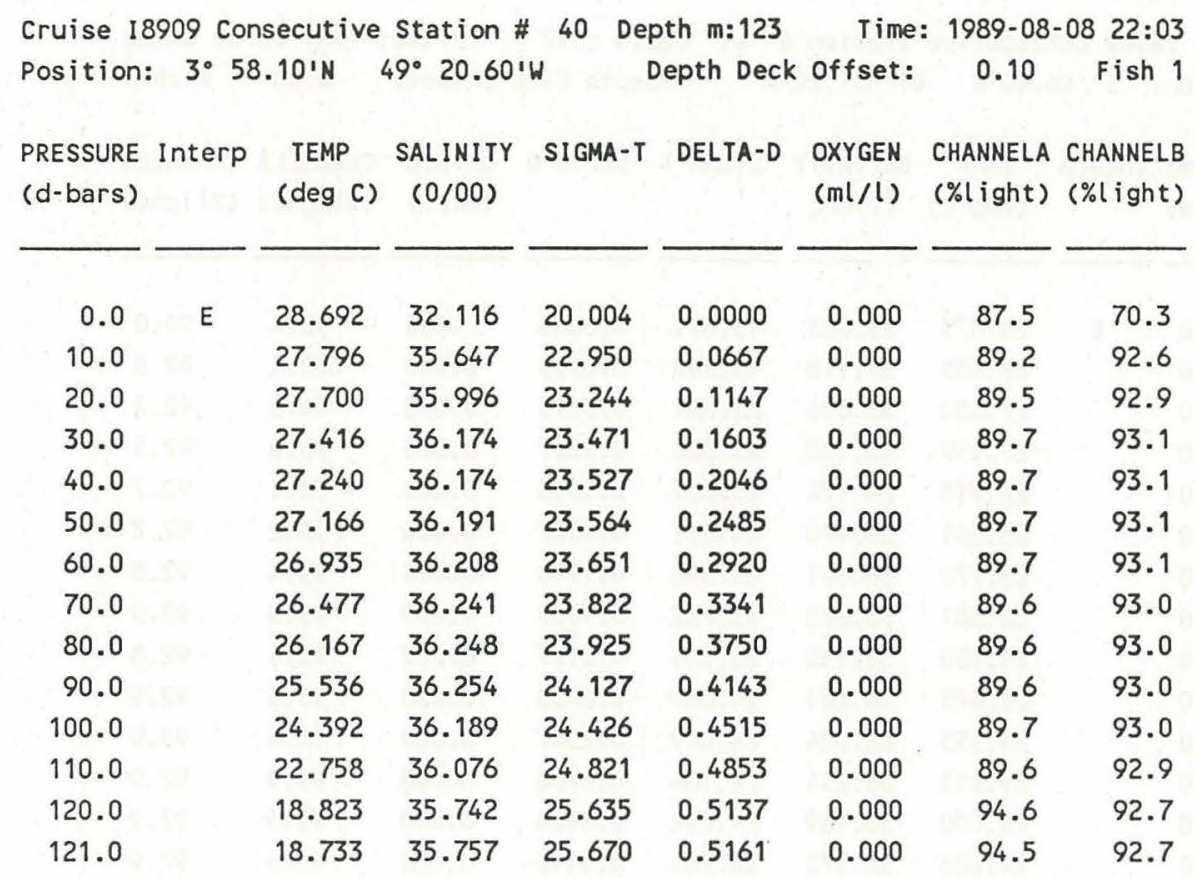

TEMPERATURE C

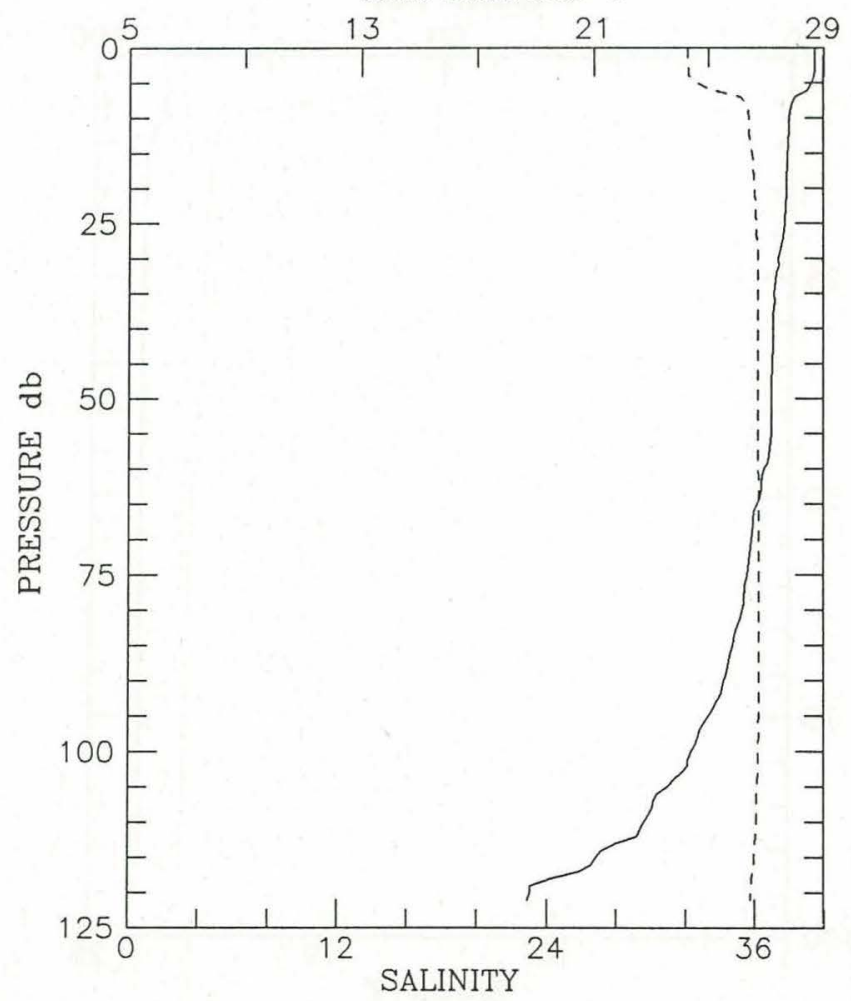

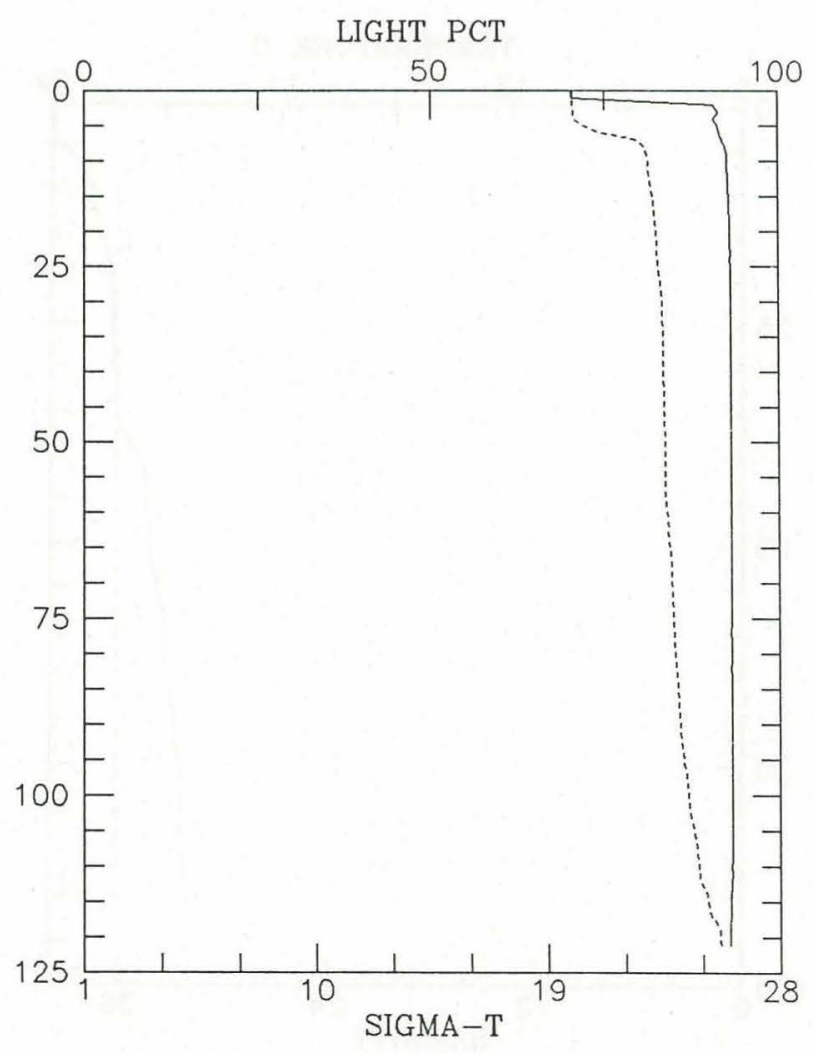




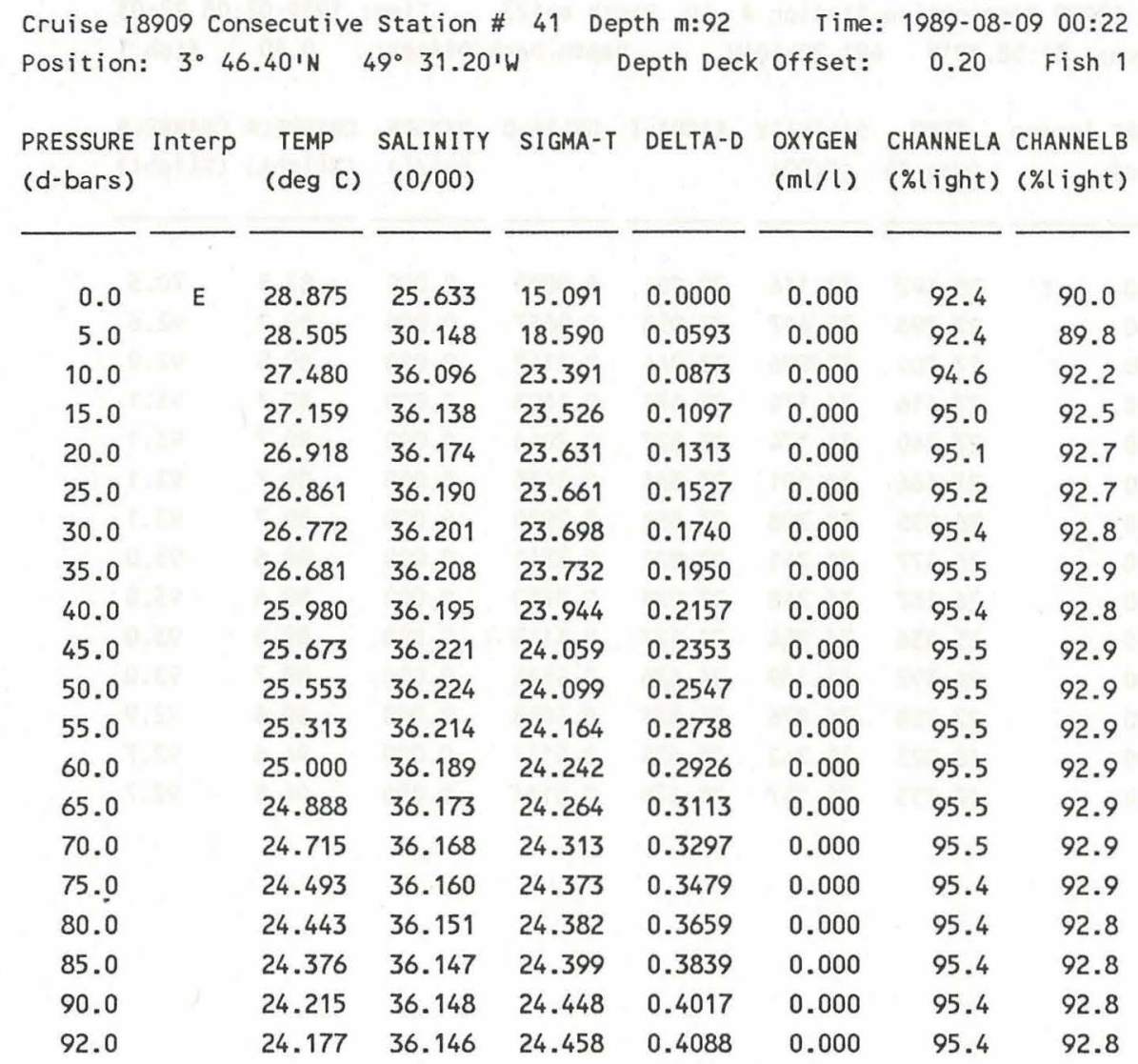
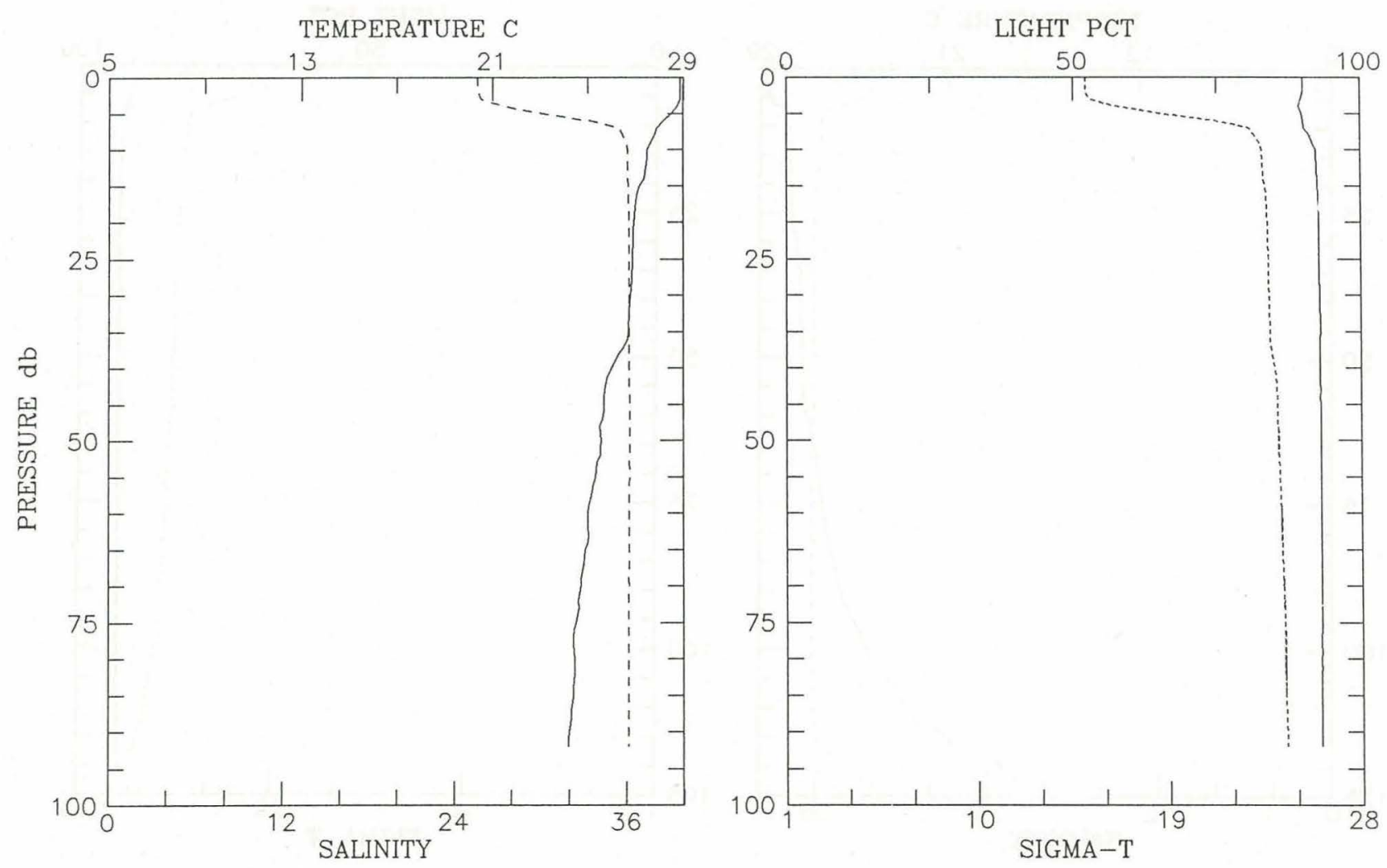


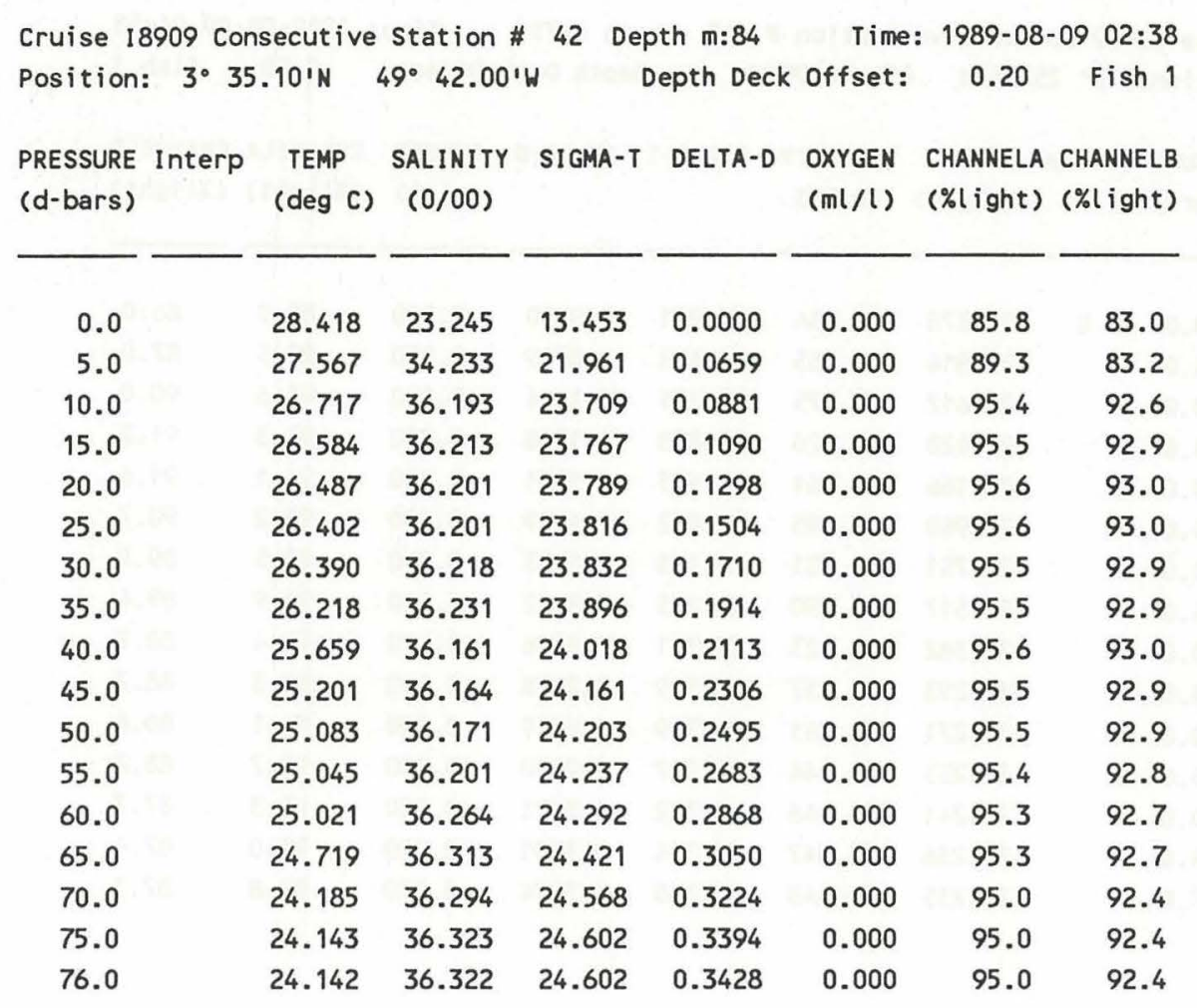
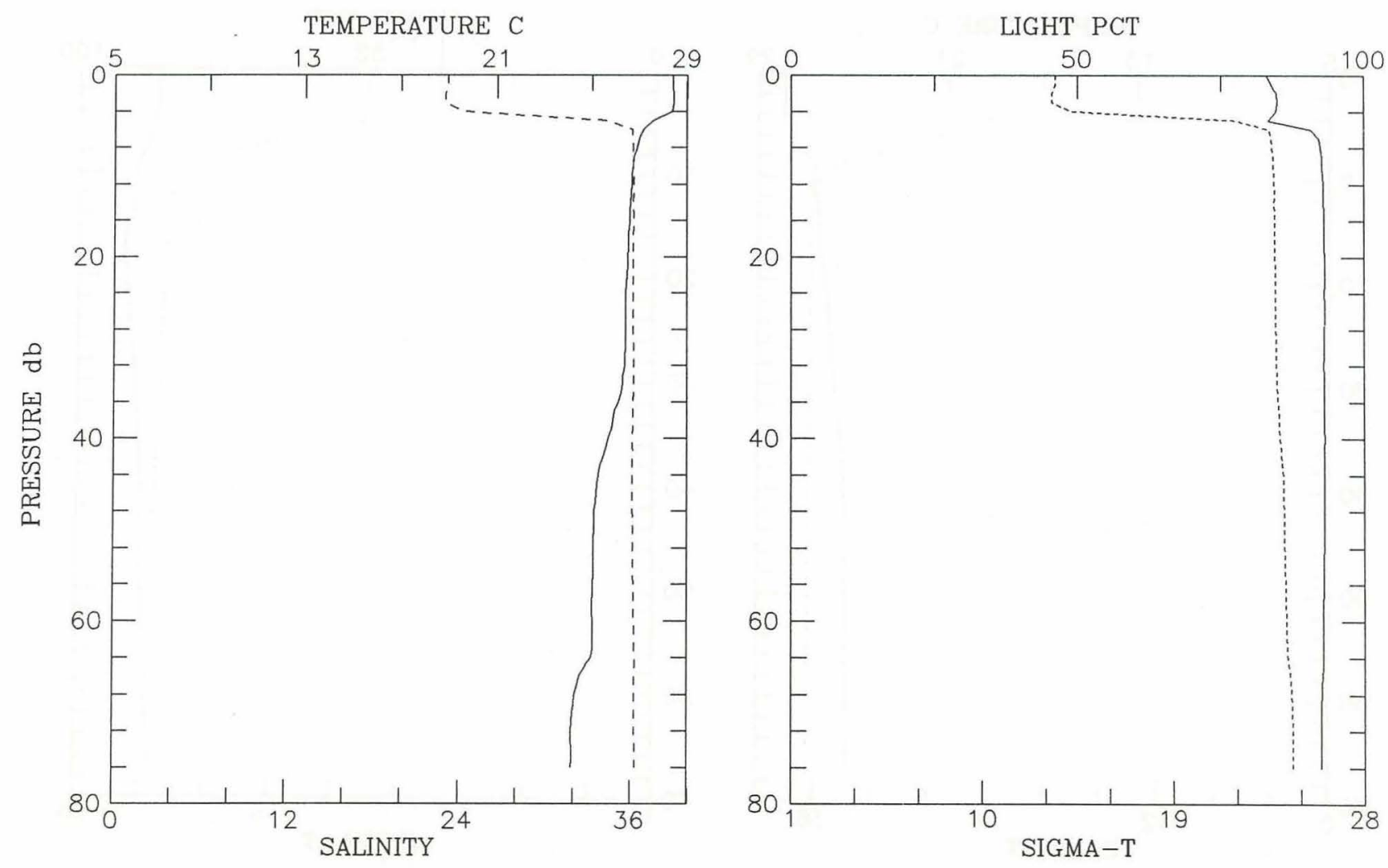


\begin{tabular}{|c|c|c|c|c|c|c|c|c|}
\hline $\begin{array}{l}\text { Cruise I } 85 \\
\text { Position: }\end{array}$ & \multicolumn{2}{|c|}{$\begin{array}{l}909 \text { Consecutive } \\
3^{\circ} 25.55^{\prime} \mathrm{N}\end{array}$} & $\begin{array}{l}\text { Station \# } \\
49^{\circ} 54.09^{\prime} \mathrm{H}\end{array}$ & \multicolumn{2}{|c|}{ Depth Deck } & $\begin{array}{l}\text { Time: } \\
\text { fset: }\end{array}$ & $\begin{array}{c}1989-08- \\
0.50\end{array}$ & $\begin{array}{r}04: 55 \\
\text { Fish } 1\end{array}$ \\
\hline $\begin{array}{l}\text { PRESSURE } \\
\text { (d-bars) }\end{array}$ & Interp & $\begin{array}{l}\text { TEMP } \\
(\operatorname{deg} C)\end{array}$ & $\begin{array}{l}\text { SALINITY } \\
(0 / 00)\end{array}$ & SIGMA-T & DELTA-D & $\begin{array}{l}\text { OXYGEN } \\
(\mathrm{ml} / \mathrm{l})\end{array}$ & $\begin{array}{l}\text { CHANNELA } \\
(\% \text { light) }\end{array}$ & $\begin{array}{l}\text { CHANNELB } \\
\text { (\%light) }\end{array}$ \\
\hline 0.0 & $E$ & 27.878 & 22.414 & 13.001 & 0.0000 & 0.000 & 88.0 & 86.0 \\
\hline 5.0 & & 27.814 & 24.555 & 14.623 & 0.0709 & 0.000 & 89.5 & 87.0 \\
\hline 10.0 & & 26.617 & 35.575 & 23.275 & 0.1114 & 0.000 & 92.6 & 90.0 \\
\hline 15.0 & & 26.420 & 36.226 & 23.828 & 0.1328 & 0.000 & 94.3 & 91.2 \\
\hline 20.0 & & 26.166 & 36.261 & 23.935 & 0.1531 & 0.000 & 94.1 & 91.6 \\
\hline 25.0 & & 25.940 & 36.295 & 24.032 & 0.1729 & 0.000 & 93.2 & 90.7 \\
\hline 30.0 & & 25.751 & 36.331 & 24.118 & 0.1923 & 0.000 & 92.5 & 89.9 \\
\hline 35.0 & & 25.517 & 36.390 & 24.235 & 0.2112 & 0.000 & 91.9 & 89.4 \\
\hline 40.0 & & 25.382 & 36.423 & 24.301 & 0.2296 & 0.000 & 91.4 & 88.9 \\
\hline 45.0 & & 25.293 & 36.437 & 24.339 & 0.2478 & 0.000 & 91.3 & 88.7 \\
\hline 50.0 & & 25.271 & 36.441 & 24.349 & 0.2659 & 0.000 & 91.1 & 88.6 \\
\hline 55.0 & & 25.253 & 36.444 & 24.357 & 0.2840 & 0.000 & 90.7 & 88.2 \\
\hline 60.0 & & 25.241 & 36.446 & 24.362 & 0.3021 & 0.000 & 90.3 & 87.7 \\
\hline 65.0 & & 25.236 & 36.447 & 24.364 & 0.3201 & 0.000 & 90.0 & 87.4 \\
\hline 67.0 & & 25.235 & 36.448 & 24.365 & 0.3274 & 0.000 & 89.8 & 87.1 \\
\hline
\end{tabular}
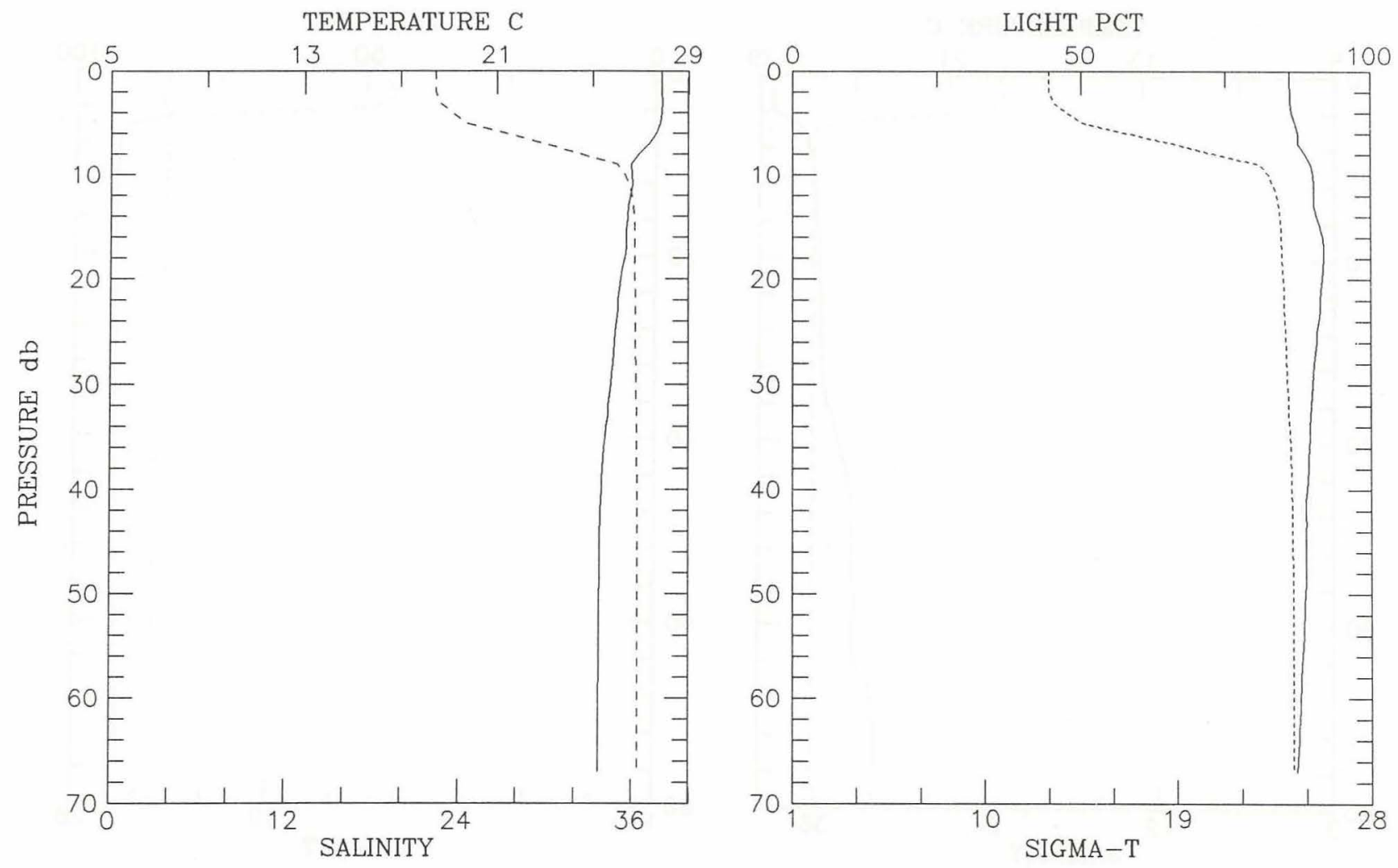


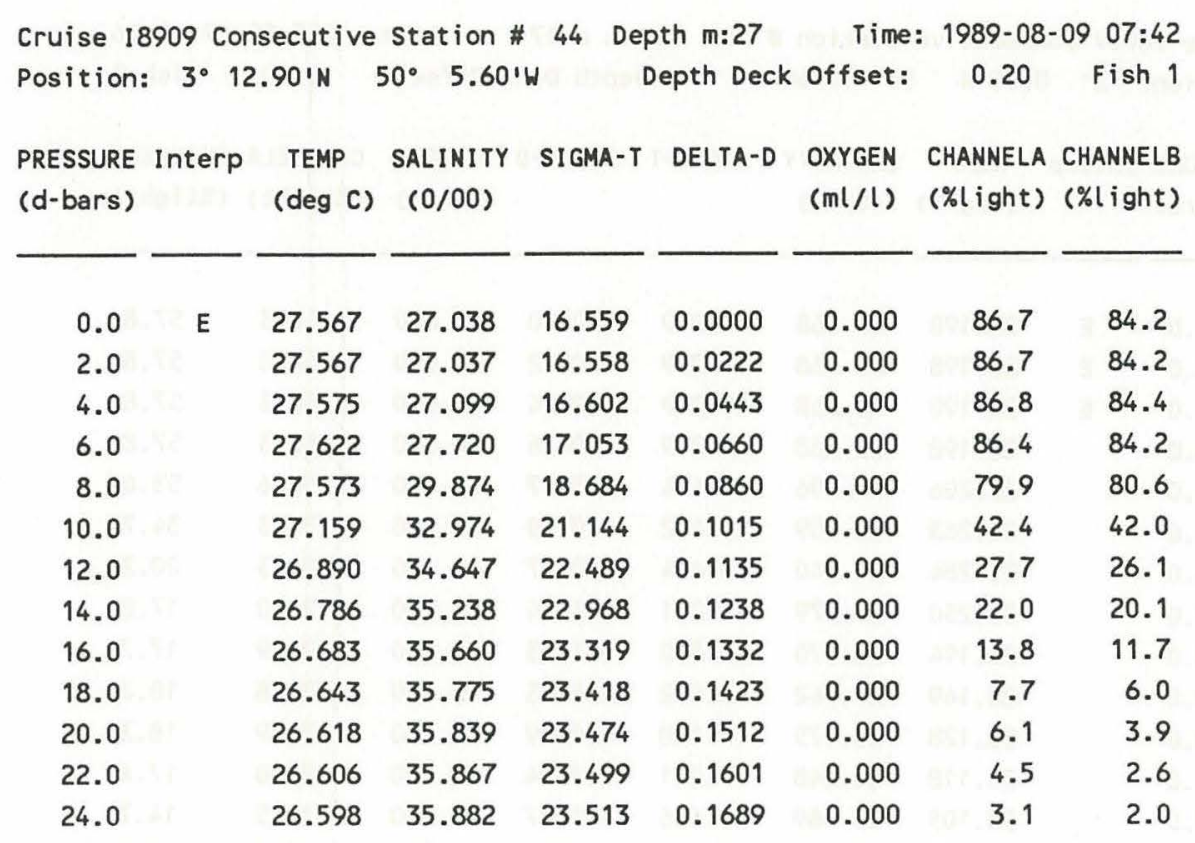
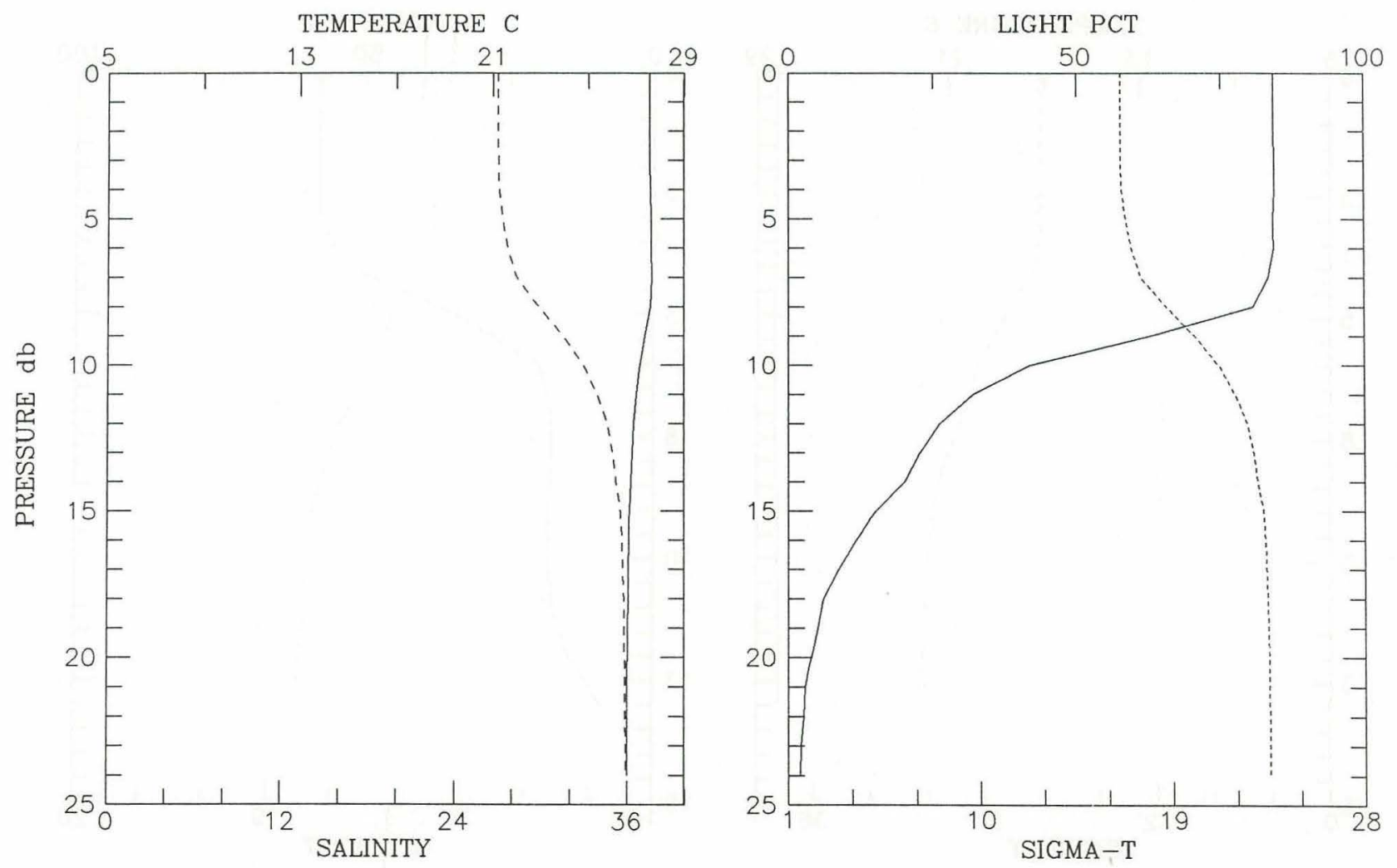


\begin{tabular}{|c|c|c|c|c|c|c|c|c|}
\hline Position: & $3^{\circ}$ & $.00 \mathrm{~N}$ & $50^{\circ} 14.60^{\prime}$ & & epth Deck & offset: & 0.20 & Fish 1 \\
\hline $\begin{array}{l}\text { PRESSURE } \\
\text { (d-bars) }\end{array}$ & Interp & $\begin{array}{c}\text { TEMP } \\
\text { (deg C) }\end{array}$ & $\begin{array}{l}\text { SALINITY } \\
(0 / 00)\end{array}$ & SIGMA-T & DELTA-D & $\begin{array}{l}\text { OXYGEN } \\
(\mathrm{ml} / \mathrm{l})\end{array}$ & $\begin{array}{l}\text { CHANNELA } \\
(\% \text { light) }\end{array}$ & $\begin{array}{l}\text { CHANNELB } \\
\text { (\%light) }\end{array}$ \\
\hline 0.0 & $E$ & 28.198 & 20.268 & 11.299 & 0.0000 & 0.000 & 58.3 & 57.8 \\
\hline 1.0 & $E$ & 28.198 & 20.268 & 11.299 & 0.0162 & 0.000 & 58.3 & 57.8 \\
\hline 2.0 & $E$ & 28.198 & 20.268 & 11.299 & 0.0324 & 0.000 & 58.3 & 57.8 \\
\hline 3.0 & & 28.198 & 20.268 & 11.299 & 0.0486 & 0.000 & 58.3 & 57.8 \\
\hline 4.0 & & 28.206 & 20.506 & 11.474 & 0.0647 & 0.000 & 52.6 & 53.8 \\
\hline 5.0 & & 28.263 & 22.709 & 13.102 & 0.0799 & 0.000 & 36.3 & 34.7 \\
\hline 6.0 & & 28.284 & 24.540 & 14.464 & 0.0937 & 0.000 & 24.3 & 20.2 \\
\hline 7.0 & & 28.250 & 25.579 & 15.251 & 0.1064 & 0.000 & 21.0 & 17.8 \\
\hline 8.0 & & 28.194 & 26.970 & 16.310 & 0.1183 & 0.000 & 20.9 & 17.7 \\
\hline 9.0 & & 28.149 & 27.742 & 16.902 & 0.1293 & 0.000 & 22.8 & 18.2 \\
\hline 10.0 & & 28.128 & 28.075 & 17.158 & 0.1399 & 0.000 & 21.9 & 18.3 \\
\hline 11.0 & & 28.118 & 28.248 & 17.291 & 0.1504 & 0.000 & 20.0 & 17.4 \\
\hline 12.0 & & 28.105 & 28.489 & 17.476 & 0.1607 & 0.000 & 14.5 & 14.7 \\
\hline 13.0 & & 28.093 & 28.753 & 17.678 & 0.1708 & 0.000 & 2.1 & 9.0 \\
\hline
\end{tabular}
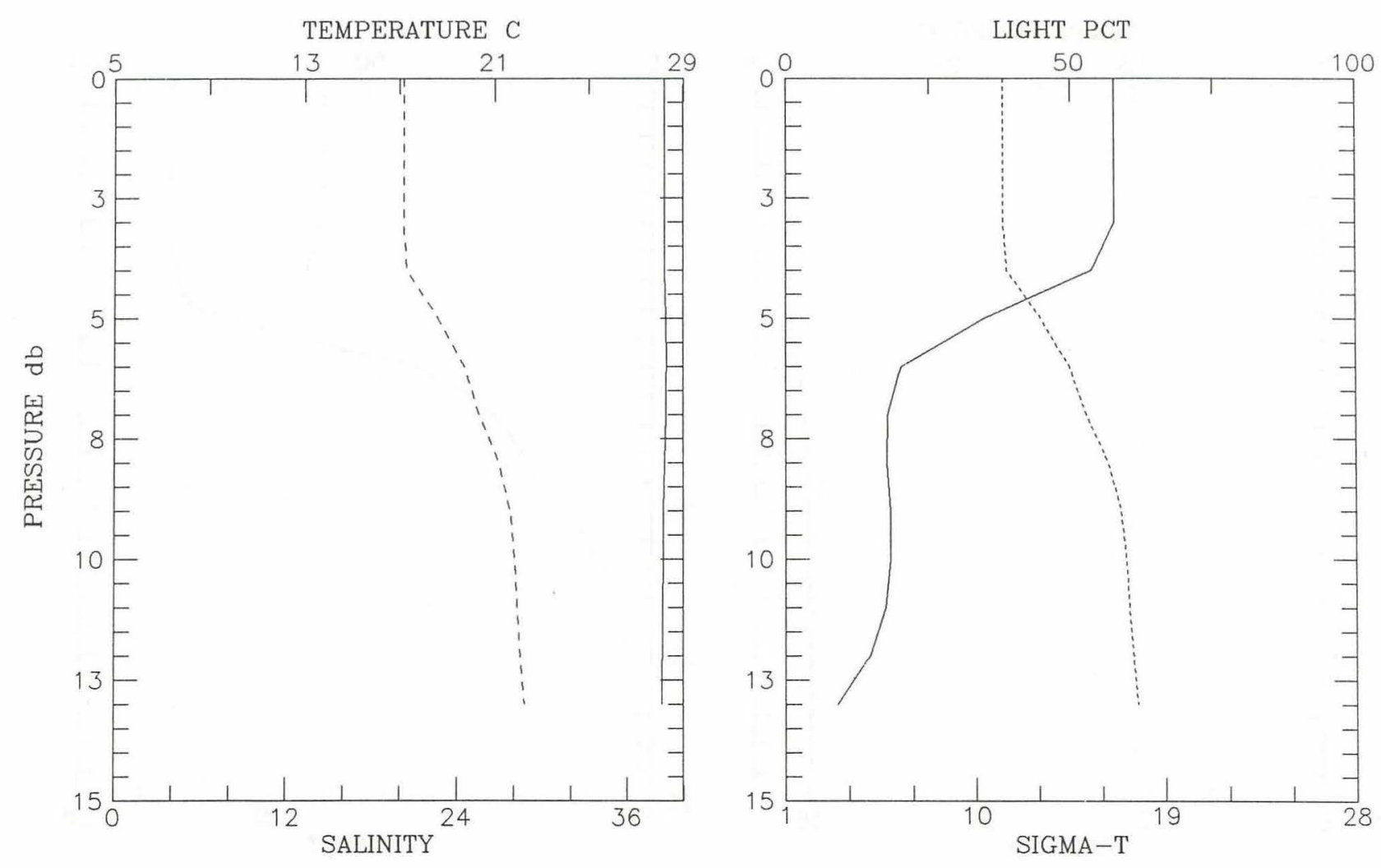


\begin{tabular}{|c|c|c|c|c|c|c|c|c|}
\hline \multirow{2}{*}{$\begin{array}{l}\text { Position: } \\
\text { PRESSURE } \\
\text { (d-bars) }\end{array}$} & \multicolumn{2}{|c|}{$=2^{\circ} 47.701 \mathrm{~N}$} & \multicolumn{2}{|c|}{$50^{\circ} 26.55^{\prime} \mathrm{W}$} & Depth Deck & Offset: & 0.20 & \multirow{2}{*}{$\begin{array}{r}\text { Fish } 1 \\
\text { CHANNELB } \\
\text { (\%light) }\end{array}$} \\
\hline & Interp & $\begin{array}{l}\text { TEMP } \\
(\operatorname{deg} C)\end{array}$ & $\begin{array}{c}\text { SALINITY } \\
(0 / 00)\end{array}$ & SIGMA-T & DELTA-D & $\begin{array}{l}\text { OXYGEN } \\
(\mathrm{ml} / \mathrm{l})\end{array}$ & $\begin{array}{l}\text { CHANNELA } \\
\text { (\%light) }\end{array}$ & \\
\hline 0.0 & $E$ & 28.393 & 15.073 & 7.365 & 0.0000 & 0.000 & 69.0 & 66.7 \\
\hline 1.0 & & 28.393 & 15.073 & 7.365 & 0.0201 & 0.000 & 69.0 & 66.7 \\
\hline 2.0 & & 28.390 & 15.073 & 7.366 & 0.0401 & 0.000 & 69.2 & 67.1 \\
\hline 3.0 & & 28.363 & 15.074 & 7.376 & 0.0602 & 0.000 & 69.6 & 67.5 \\
\hline 4.0 & & 28.311 & 15.107 & 7.416 & 0.0802 & 0.000 & 70.5 & 66.6 \\
\hline 5.0 & & 28.338 & 15.092 & 7.396 & 0.1002 & 0.000 & 70.2 & 66.8 \\
\hline 6.0 & & 28.283 & 15.132 & 7.443 & 0.1202 & 0.000 & 73.3 & 67.7 \\
\hline 7.0 & & 28.261 & 15.189 & 7.492 & 0.1402 & 0.000 & 74.3 & 70.3 \\
\hline 8.0 & & 28.300 & 15.406 & 7.642 & 0.1600 & 0.000 & 69.6 & 68.7 \\
\hline 9.0 & & 28.332 & 15.824 & 7.944 & 0.1797 & 0.000 & 54.9 & 52.8 \\
\hline 10.0 & & 28.351 & 15.992 & 8.063 & 0.1991 & 0.000 & 48.1 & 46.1 \\
\hline 11.0 & & 28.399 & 16.795 & 8.647 & 0.2182 & 0.000 & 46.6 & 40.4 \\
\hline 12.0 & & 28.450 & 18.107 & 9.609 & 0.2365 & 0.000 & 32.5 & 36.4 \\
\hline 13.0 & & 28.472 & 19.873 & 10.919 & 0.2537 & 0.000 & 0.2 & 1.7 \\
\hline
\end{tabular}
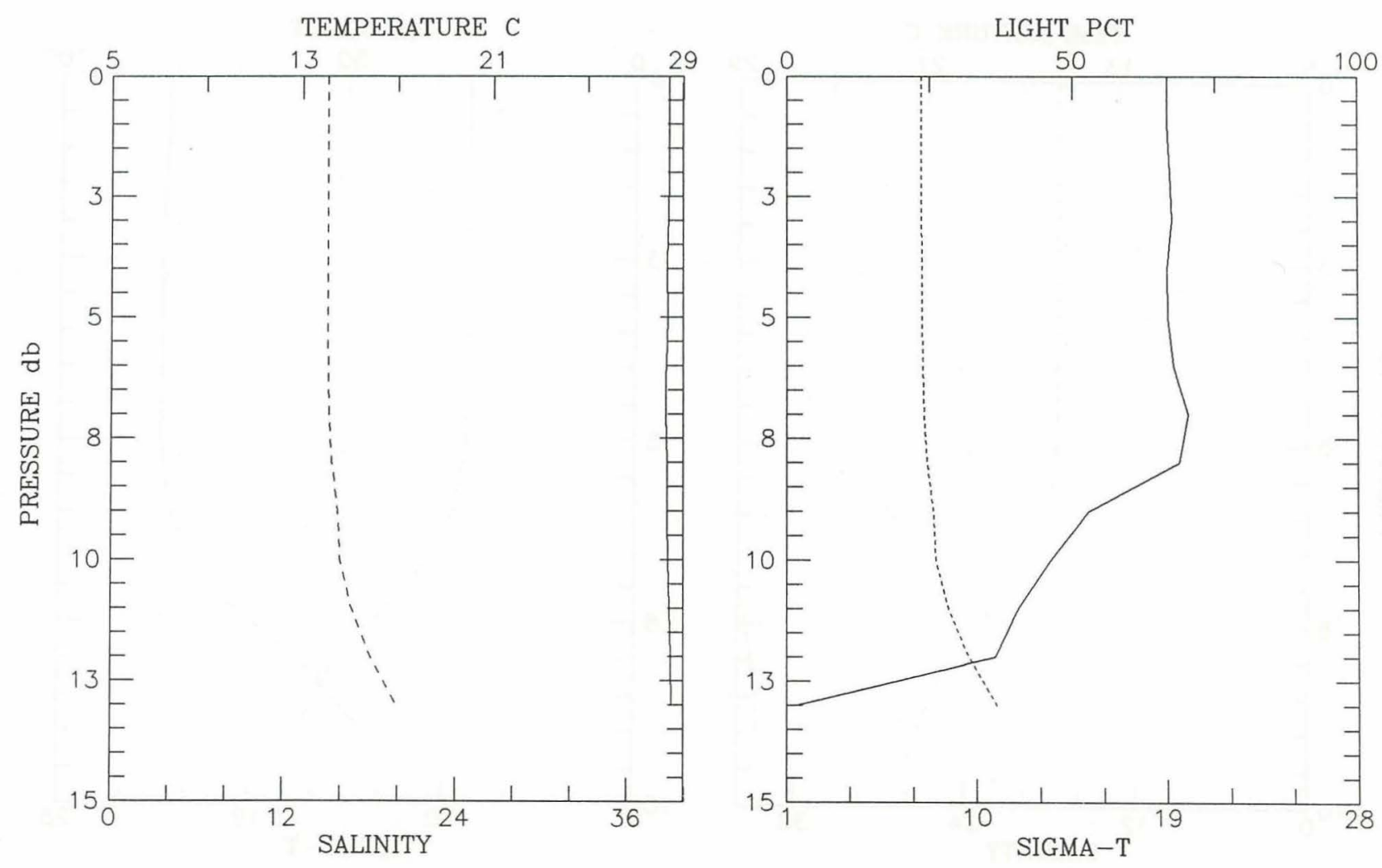


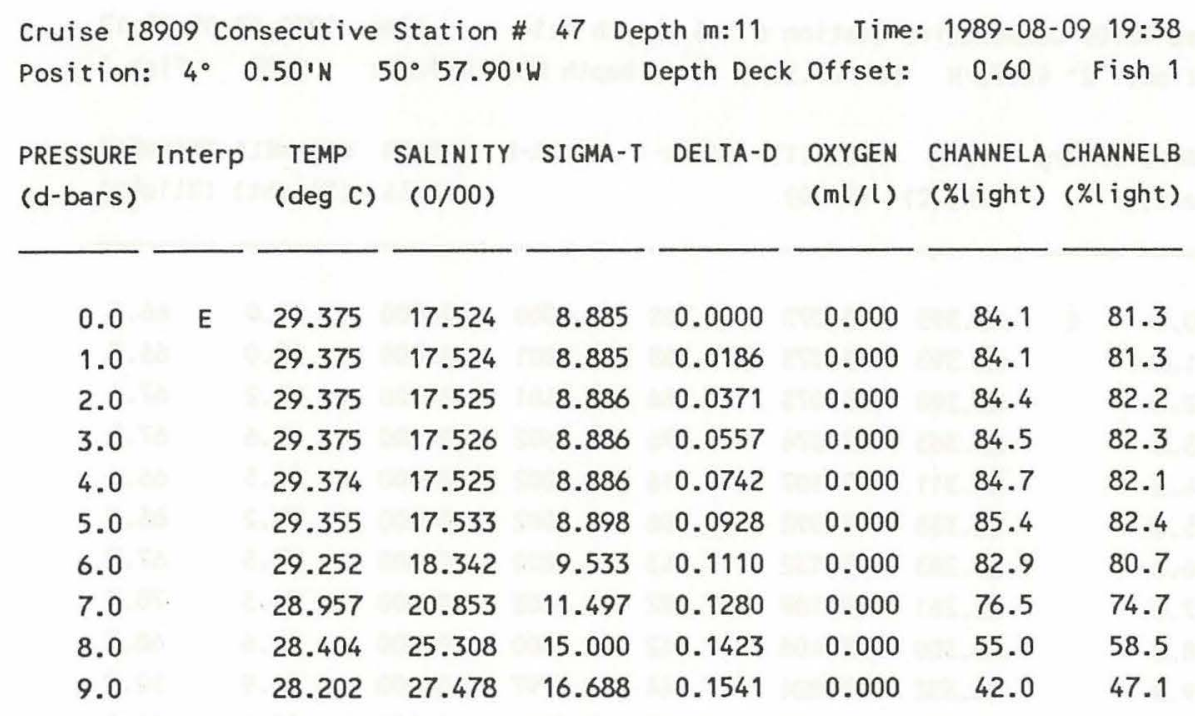

TEMPERATURE C

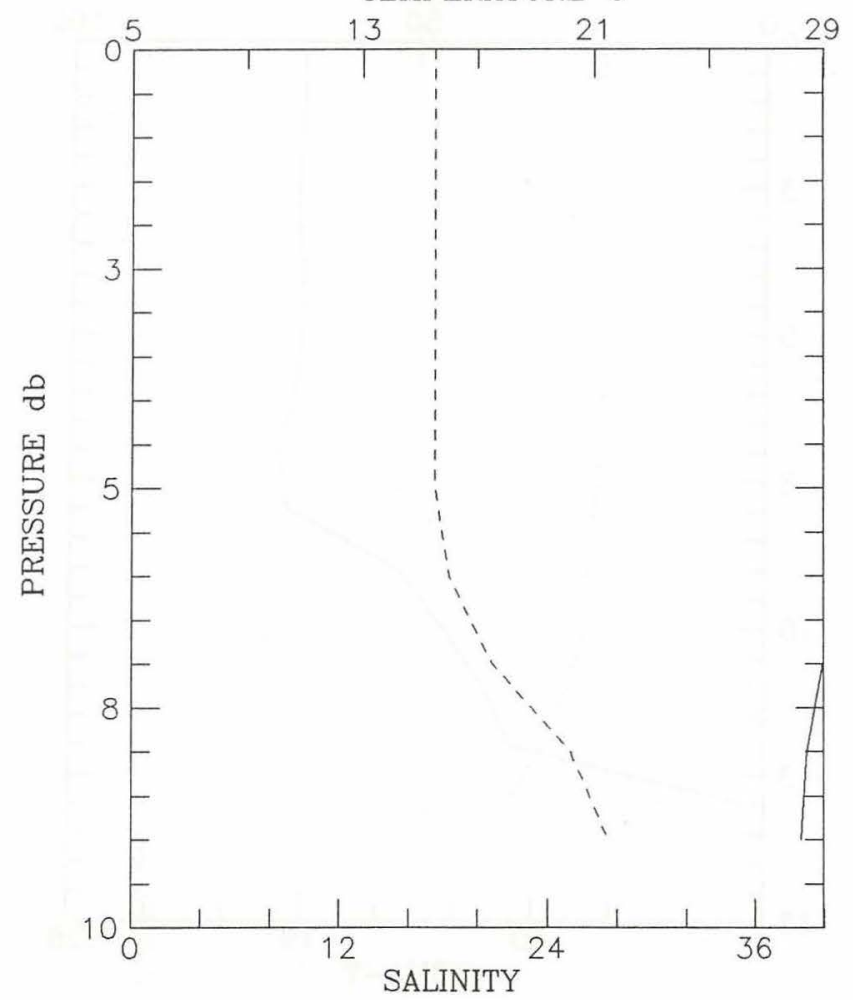

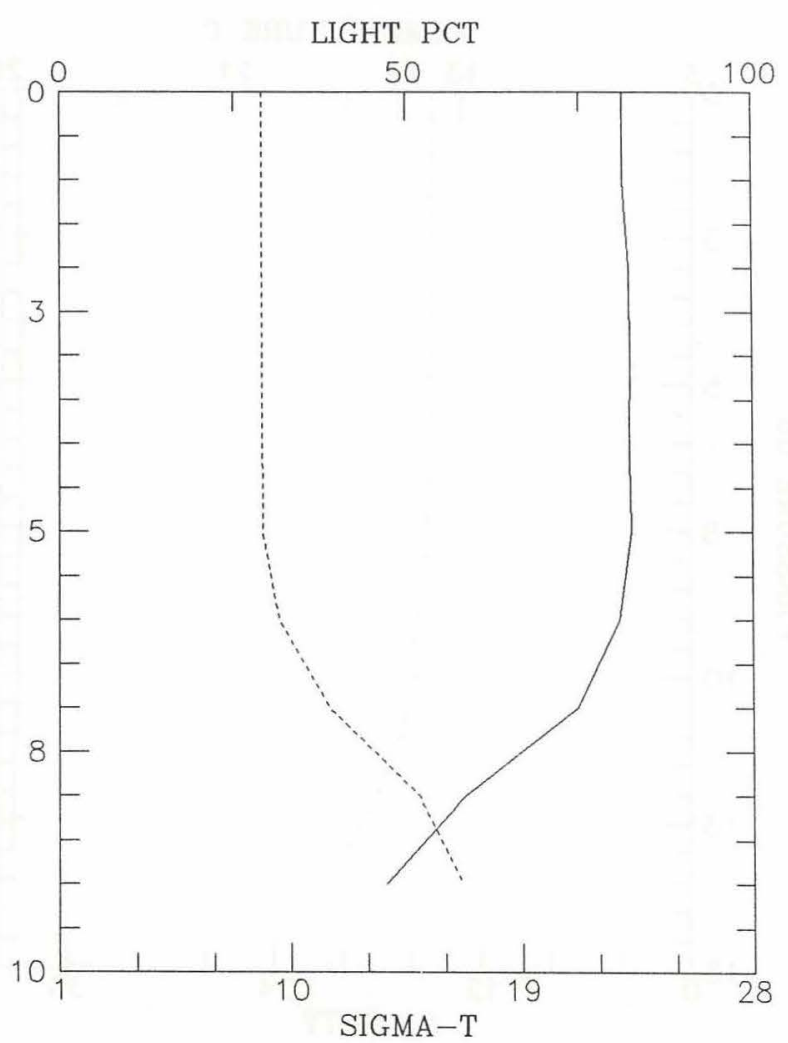




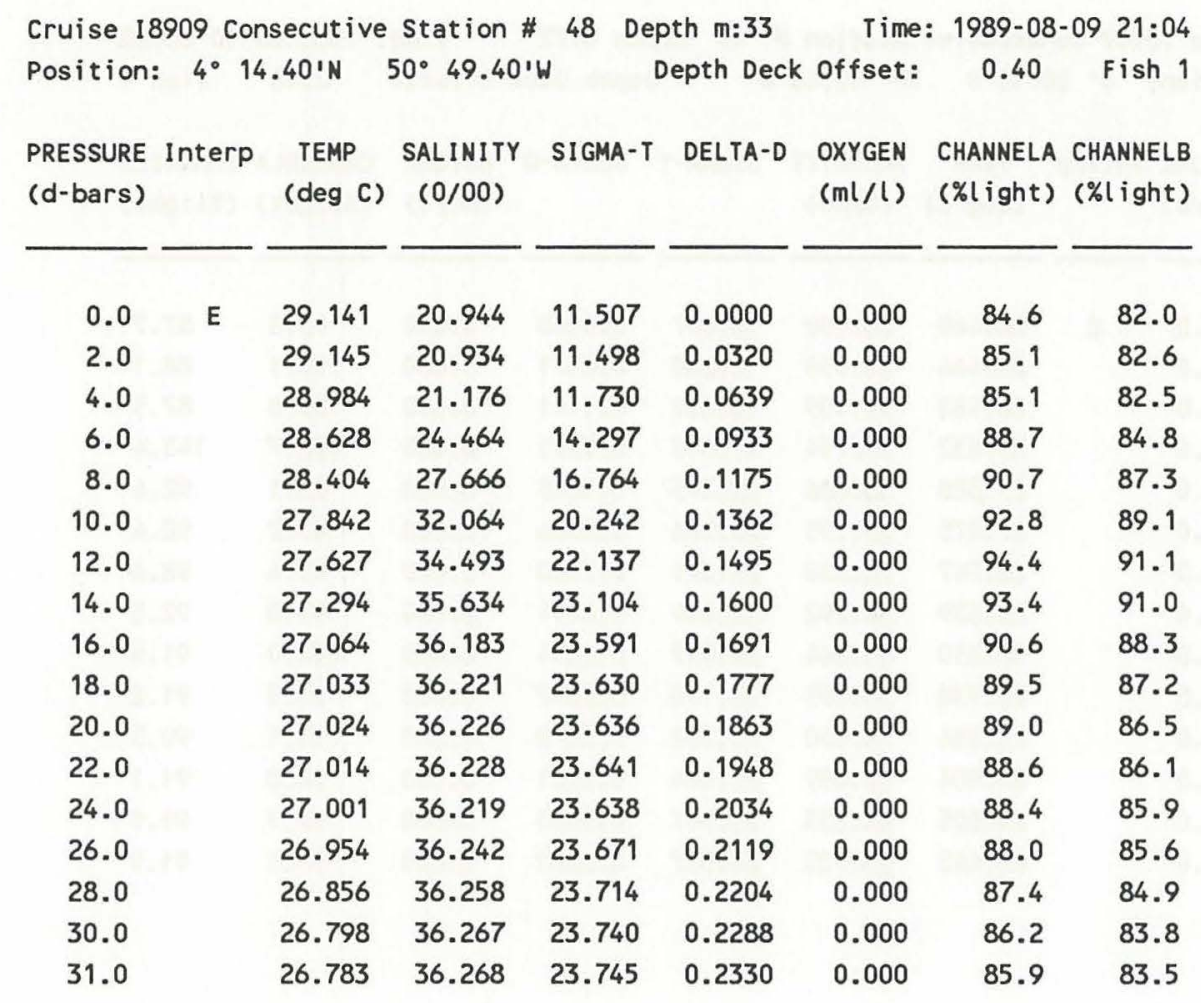
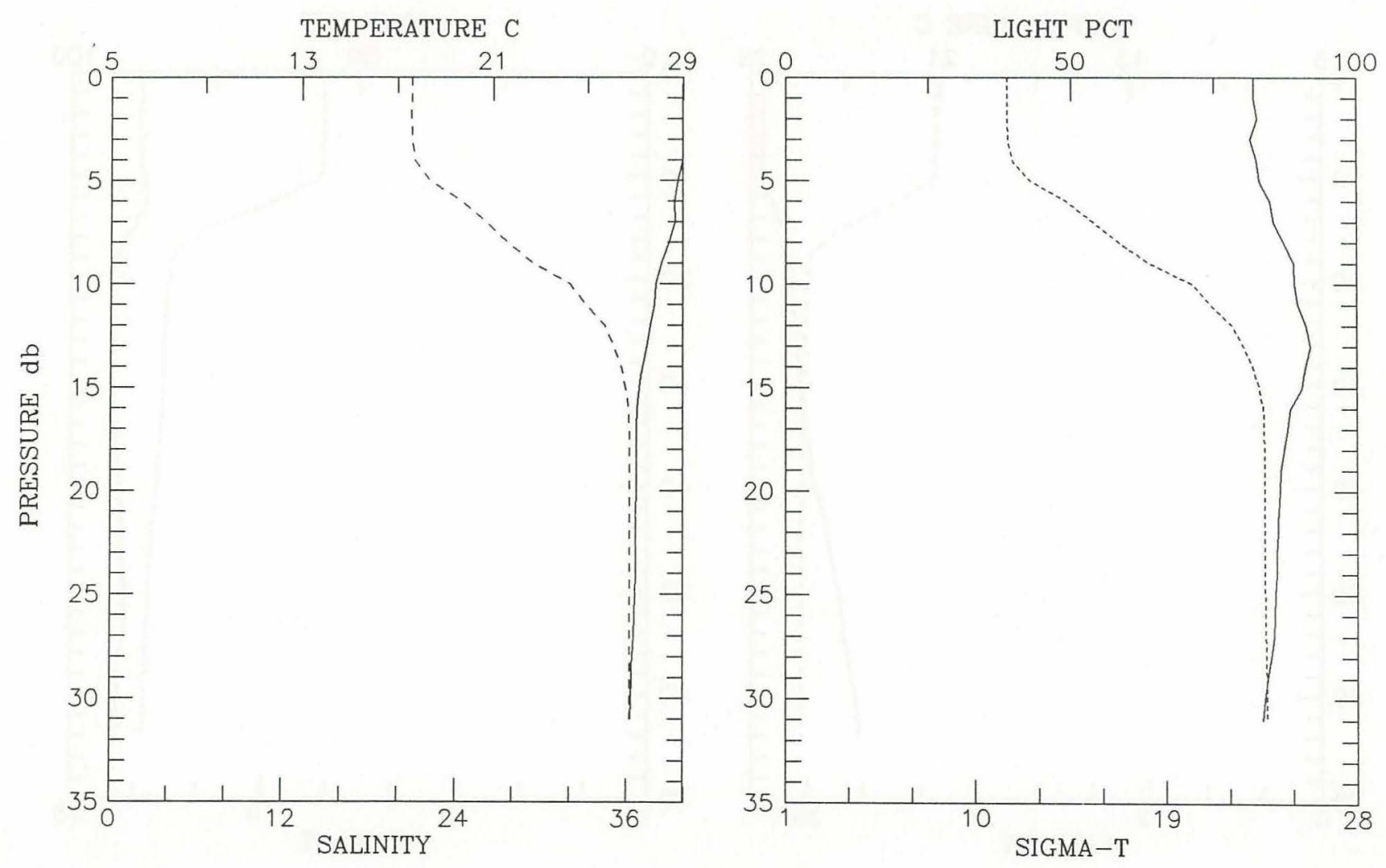


\begin{tabular}{|c|c|c|c|c|c|c|c|c|}
\hline \multirow{2}{*}{$\begin{array}{l}\text { Position: } \\
\text { PRESSURE } \\
\text { (d-bars) }\end{array}$} & \multicolumn{2}{|c|}{$=4^{\circ} 20.10^{\prime} \mathrm{N}$} & \multicolumn{2}{|c|}{$50^{\circ} 42.40^{\prime} \mathrm{W}$} & Depth Deck & Offset: & -0.10 & Fish \\
\hline & Interp & $\begin{array}{c}\text { TEMP } \\
(\operatorname{deg} C)\end{array}$ & $\begin{array}{l}\text { SALINITY } \\
(0 / 00)\end{array}$ & SIGMA-T & DELTA-D & $\begin{array}{l}\text { OXYGEN } \\
(\mathrm{ml} / \mathrm{l})\end{array}$ & $\begin{array}{l}\text { CHANNELA } \\
\text { (\%light) }\end{array}$ & $\begin{array}{l}\text { CHANNELB } \\
\text { (\%light) }\end{array}$ \\
\hline 0.0 & $E$ & 28.469 & 26.950 & 16.207 & 0.0000 & 0.000 & 90.3 & 87.7 \\
\hline 5.0 & & 28.466 & 26.950 & 16.208 & 0.0571 & 0.000 & 90.5 & 88.1 \\
\hline 10.0 & & 28.583 & 27.109 & 16.289 & 0.1141 & 0.000 & 89.8 & 87.5 \\
\hline 15.0 & & 27.632 & 33.794 & 21.610 & 0.1591 & 0.000 & 92.7 & 143.4 \\
\hline 20.0 & & 27.328 & 36.036 & 23.395 & 0.1845 & 0.000 & 95.1 & 92.4 \\
\hline 25.0 & & 27.075 & 36.195 & 23.596 & 0.2066 & 0.000 & 95.2 & 92.4 \\
\hline 30.0 & & 26.947 & 36.239 & 23.671 & 0.2280 & 0.000 & 95.4 & 92.6 \\
\hline 35.0 & & 26.639 & 36.292 & 23.809 & 0.2491 & 0.000 & 95.0 & 92.5 \\
\hline 40.0 & & 26.359 & 36.344 & 23.937 & 0.2694 & 0.000 & 94.0 & 91.5 \\
\hline 45.0 & & 25.738 & 36.395 & 24.170 & 0.2887 & 0.000 & 93.3 & 91.2 \\
\hline 50.0 & & 25.284 & 36.450 & 24.352 & 0.3072 & 0.000 & 93.1 & 90.5 \\
\hline 55.0 & & 25.004 & 36.459 & 24.444 & 0.3251 & 0.000 & 94.0 & 91.1 \\
\hline 60.0 & & 24.605 & 36.433 & 24.547 & 0.3425 & 0.000 & 94.7 & 91.9 \\
\hline 64.0 & & 24.445 & 36.423 & 24.587 & 0.3562 & 0.000 & 94.5 & 91.9 \\
\hline
\end{tabular}
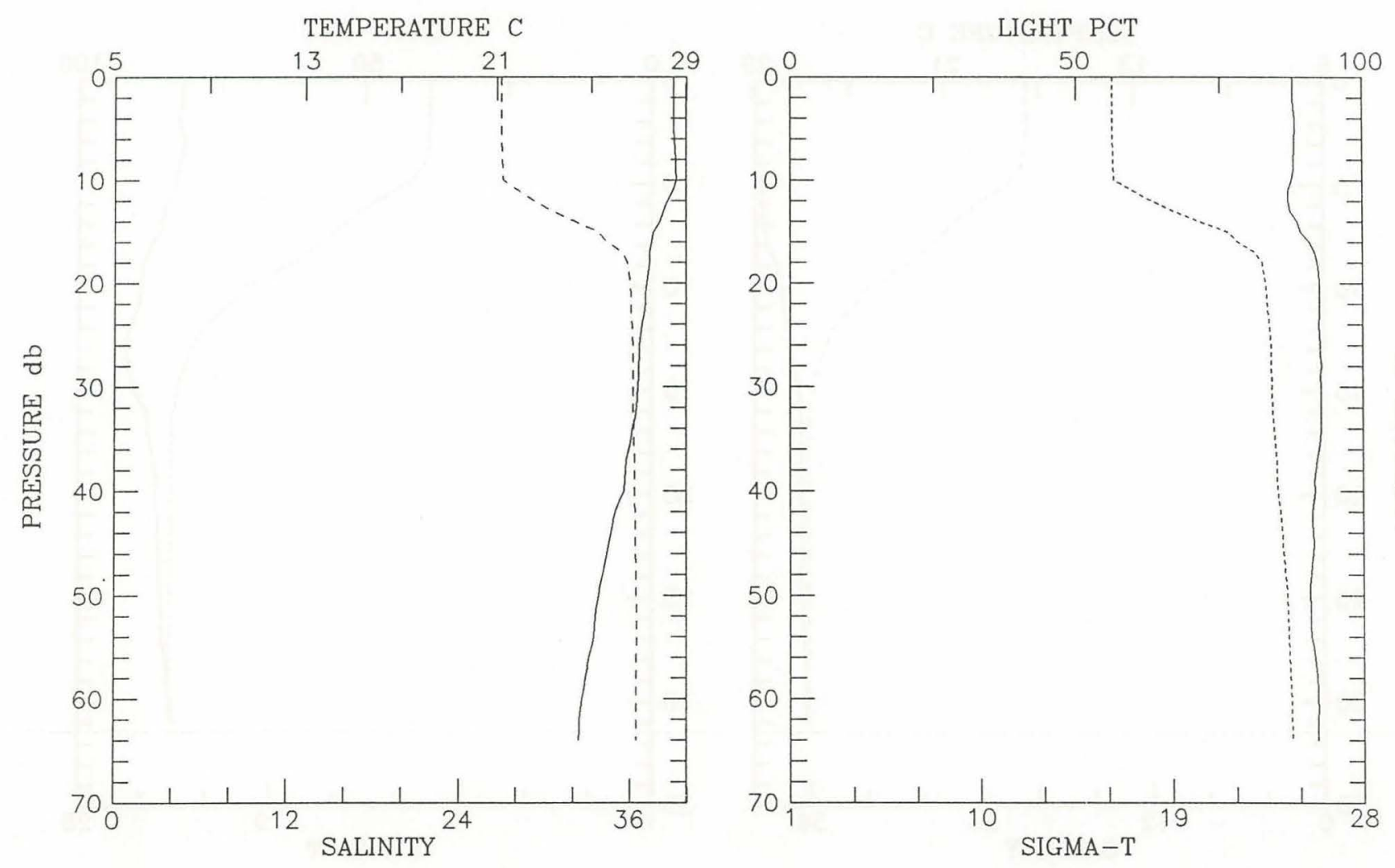


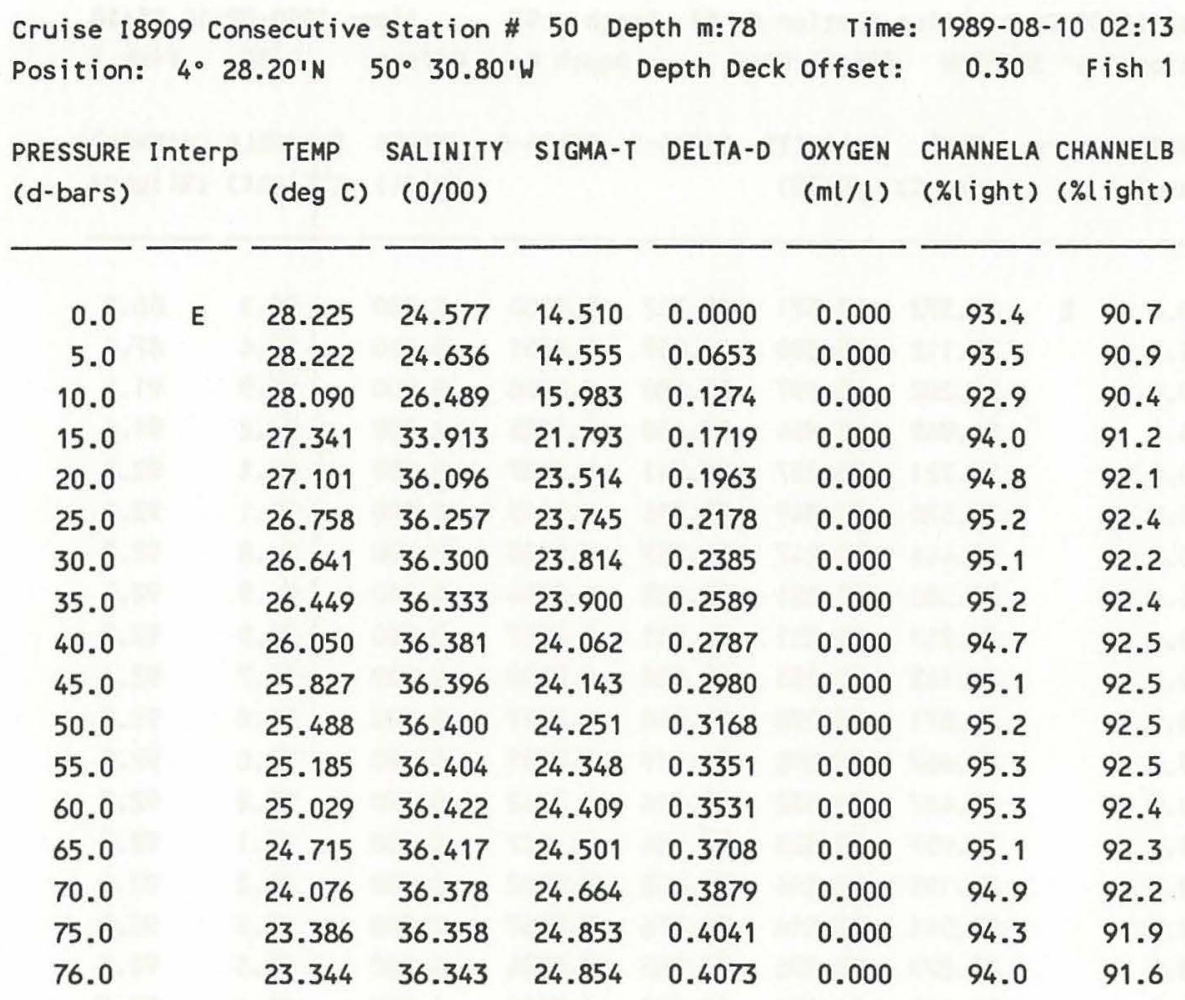

TEMPERATURE C

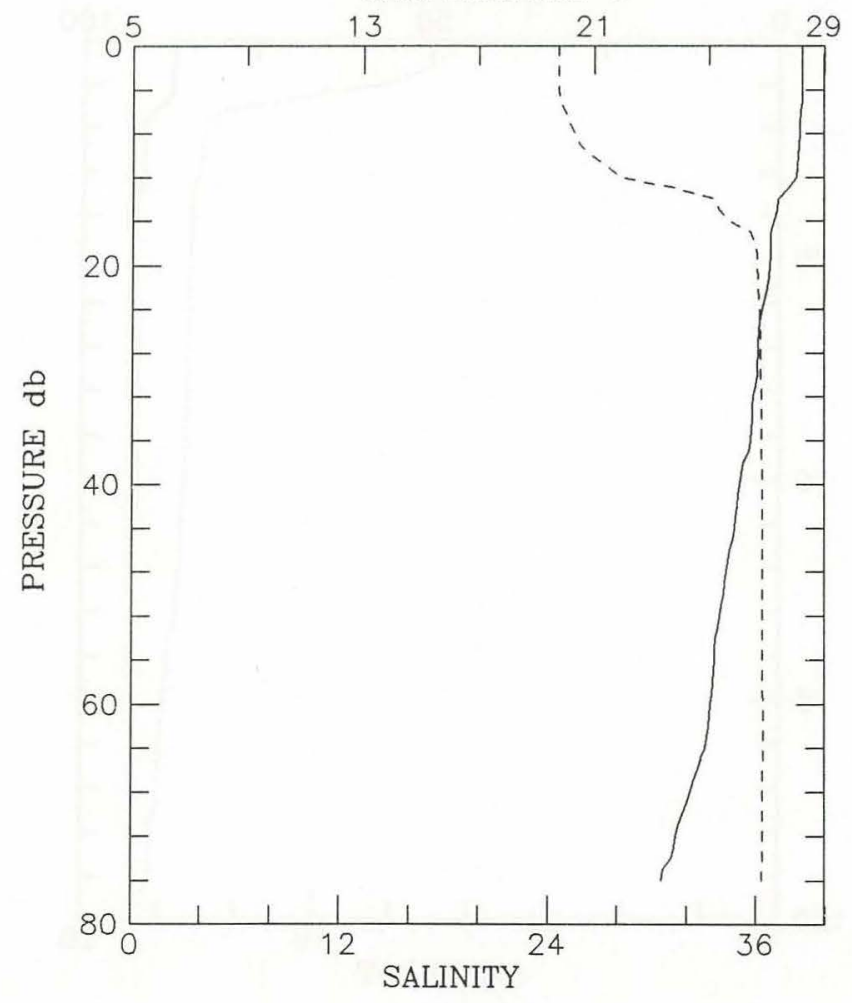

LIGHT PCT

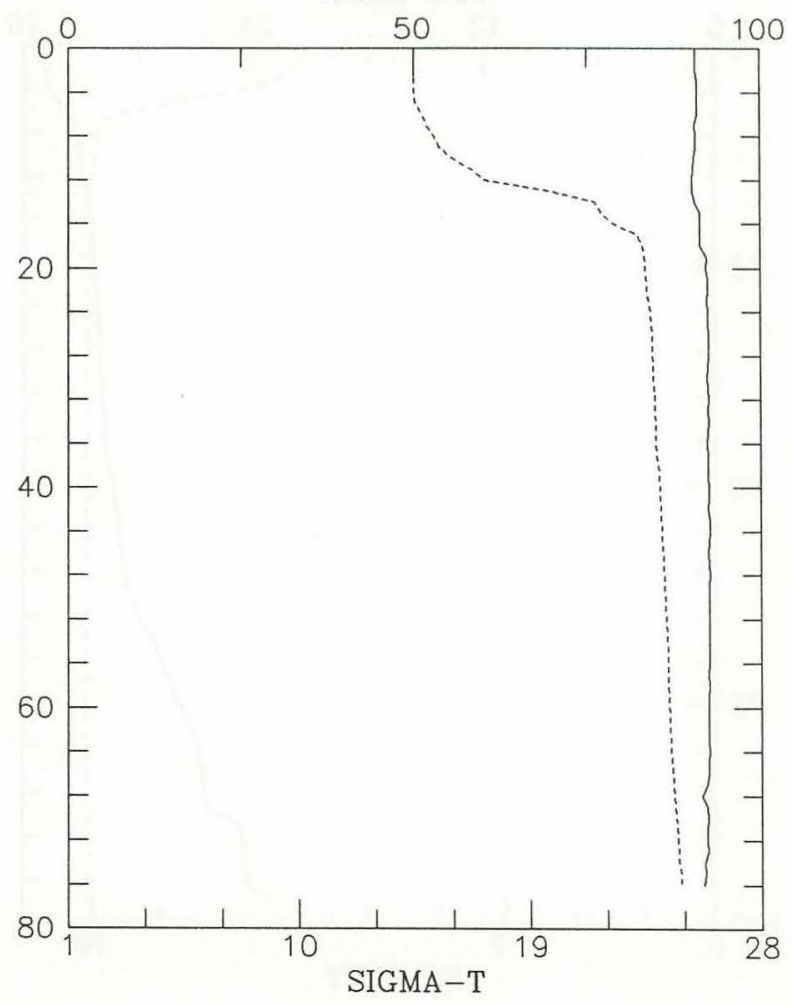


Cruise 18909 Consecutive Station \# 51 Depth m:97 Time: 1989-08-10 05:18 Position: $4^{\circ} 39.50^{\prime} \mathrm{N} 50^{\circ} 23.00^{\prime} \mathrm{W} \quad$ Depth Deck offset: $0.30 \quad \mathrm{Fish} 1$

\begin{tabular}{|c|c|c|c|c|c|c|c|c|}
\hline $\begin{array}{l}\text { PRESSURE } \\
\text { (d-bars) }\end{array}$ & Interp & $\begin{array}{c}\text { TEMP } \\
(\operatorname{deg} C)\end{array}$ & $\begin{array}{c}\text { SALINITY } \\
(0 / 00)\end{array}$ & SIGMA-T & DELTA-D & $\begin{array}{l}\text { OXYGEN } \\
(\mathrm{ml} / \mathrm{l})\end{array}$ & $\begin{array}{l}\text { CHANNELA } \\
\text { (\%light) }\end{array}$ & $\begin{array}{l}\text { CHANNELB } \\
\text { (\%light) }\end{array}$ \\
\hline 0.0 & E & 28.372 & 23.571 & 13.712 & 0.0000 & 0.000 & 89.3 & 86.8 \\
\hline 5.0 & & 28.112 & 26.880 & 16.269 & 0.0651 & 0.000 & 89.4 & 87.1 \\
\hline 10.0 & & 27.202 & 35.697 & 23.180 & 0.0996 & 0.000 & 93.9 & 91.1 \\
\hline 15.0 & & 26.965 & 35.954 & 23.450 & 0.1225 & 0.000 & 94.2 & 91.4 \\
\hline 20.0 & & 26.721 & 36.237 & 23.741 & 0.1437 & 0.000 & 95.1 & 92.3 \\
\hline 25.0 & & 26.520 & 36.249 & 23.814 & 0.1645 & 0.000 & 95.1 & 92.4 \\
\hline 30.0 & & 26.444 & 36.247 & 23.837 & 0.1850 & 0.000 & 94.8 & 92.2 \\
\hline 35.0 & & 26.306 & 36.251 & 23.883 & 0.2054 & 0.000 & 94.9 & 92.1 \\
\hline 40.0 & & 26.247 & 36.251 & 23.901 & 0.2257 & 0.000 & 94.9 & 92.2 \\
\hline 45.0 & & 26.142 & 36.253 & 23.936 & 0.2459 & 0.000 & 94.7 & 92.1 \\
\hline 50.0 & & 25.871 & 36.278 & 24.040 & 0.2657 & 0.000 & 94.8 & 92.0 \\
\hline 55.0 & & 25.667 & 36.298 & 24.119 & 0.2851 & 0.000 & 95.0 & 92.2 \\
\hline 60.0 & & 25.467 & 36.332 & 24.206 & 0.3042 & 0.000 & 95.0 & 92.3 \\
\hline 65.0 & & 24.957 & 36.333 & 24.364 & 0.3227 & 0.000 & 95.1 & 92.3 \\
\hline 70.0 & & 24.195 & 36.344 & 24.603 & 0.3402 & 0.000 & 95.2 & 92.4 \\
\hline 75.0 & & 23.541 & 36.316 & 24.776 & 0.3567 & 0.000 & 95.3 & 92.5 \\
\hline 80.0 & & 22.879 & 36.296 & 24.953 & 0.3724 & 0.000 & 95.3 & 92.5 \\
\hline 85.0 & & 22.688 & 36.285 & 25.000 & 0.3876 & 0.000 & 95.4 & 92.7 \\
\hline 90.0 & & 21.342 & 36.184 & 25.303 & 0.4020 & 0.000 & 95.5 & 92.7 \\
\hline 95.0 & & 21.222 & 36.174 & 25.328 & 0.4156 & 0.000 & 95.4 & 92.6 \\
\hline 97.0 & & 20.322 & 36.198 & 25.591 & 0.4208 & 0.000 & 95.3 & 92.5 \\
\hline
\end{tabular}
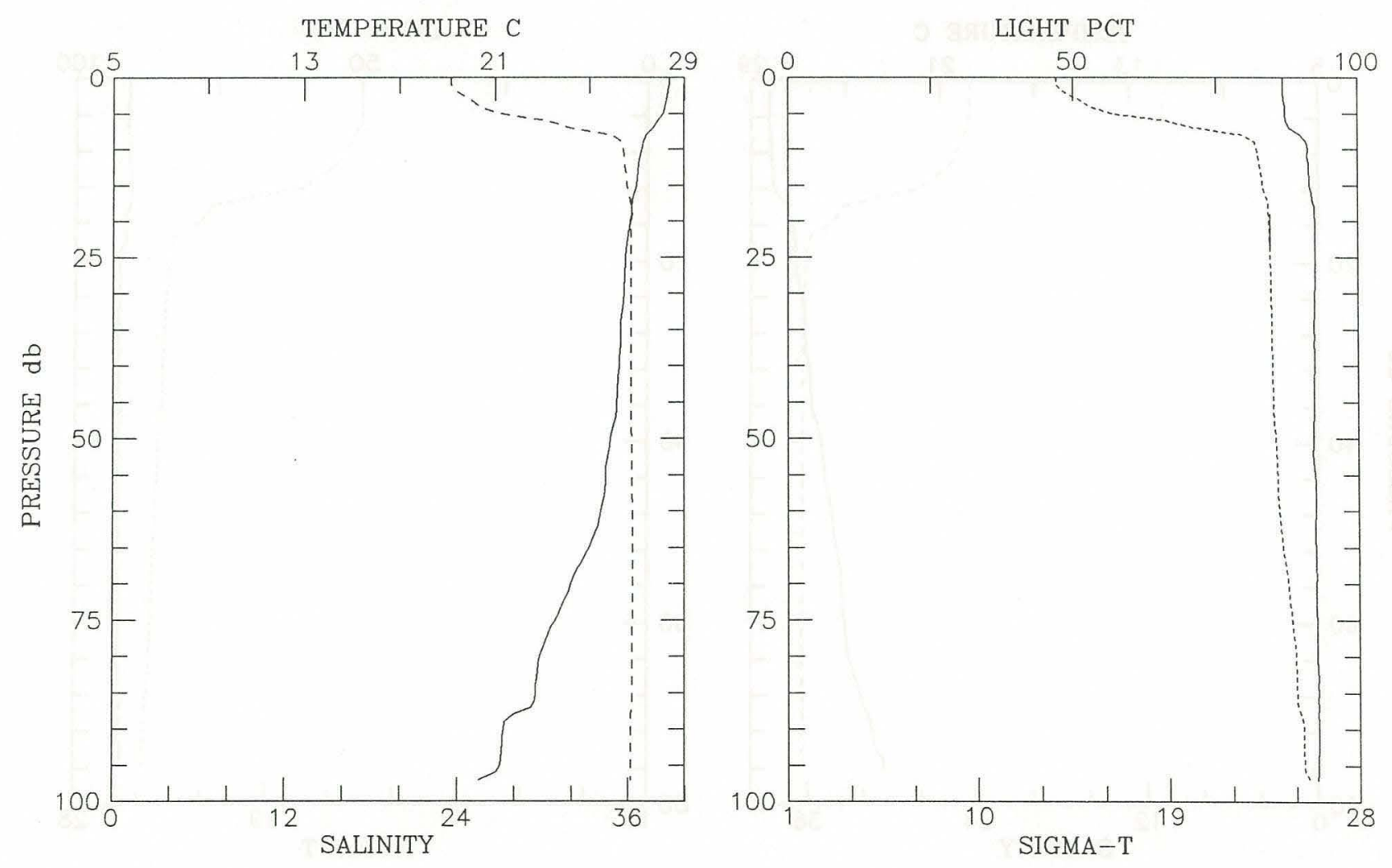


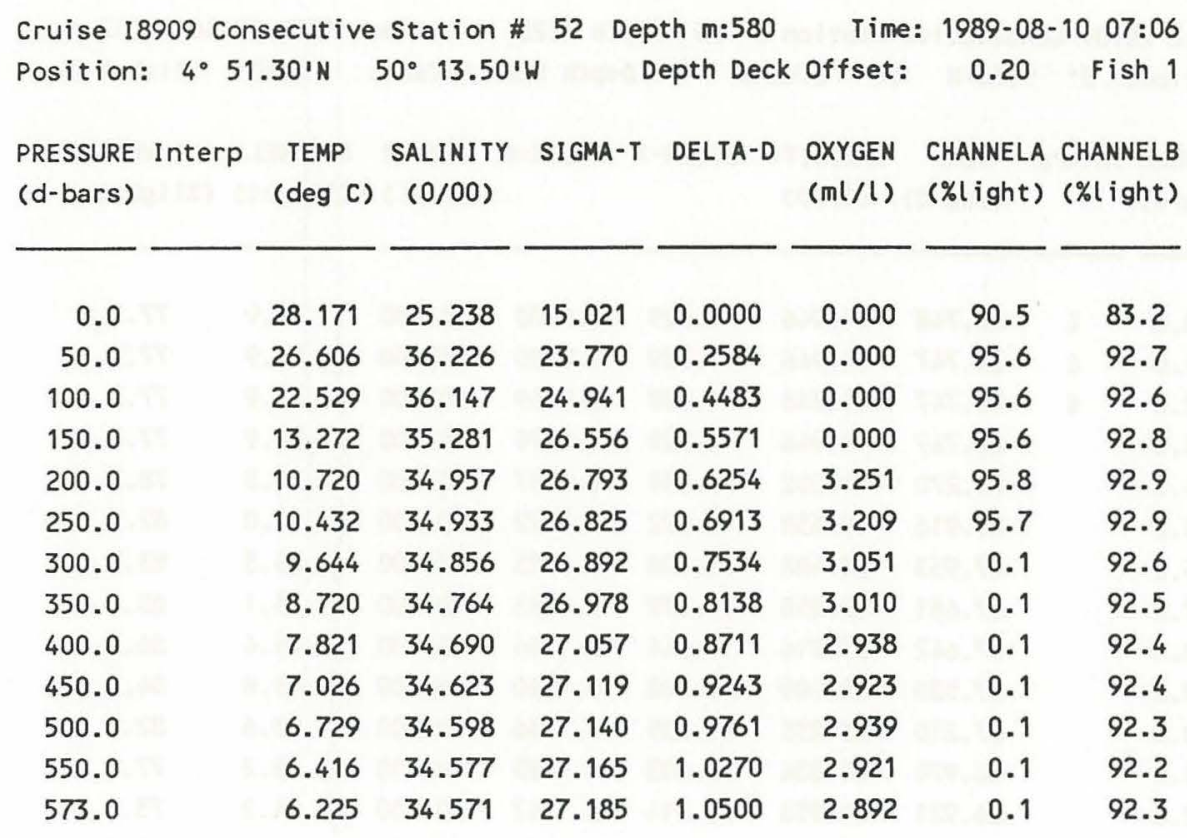
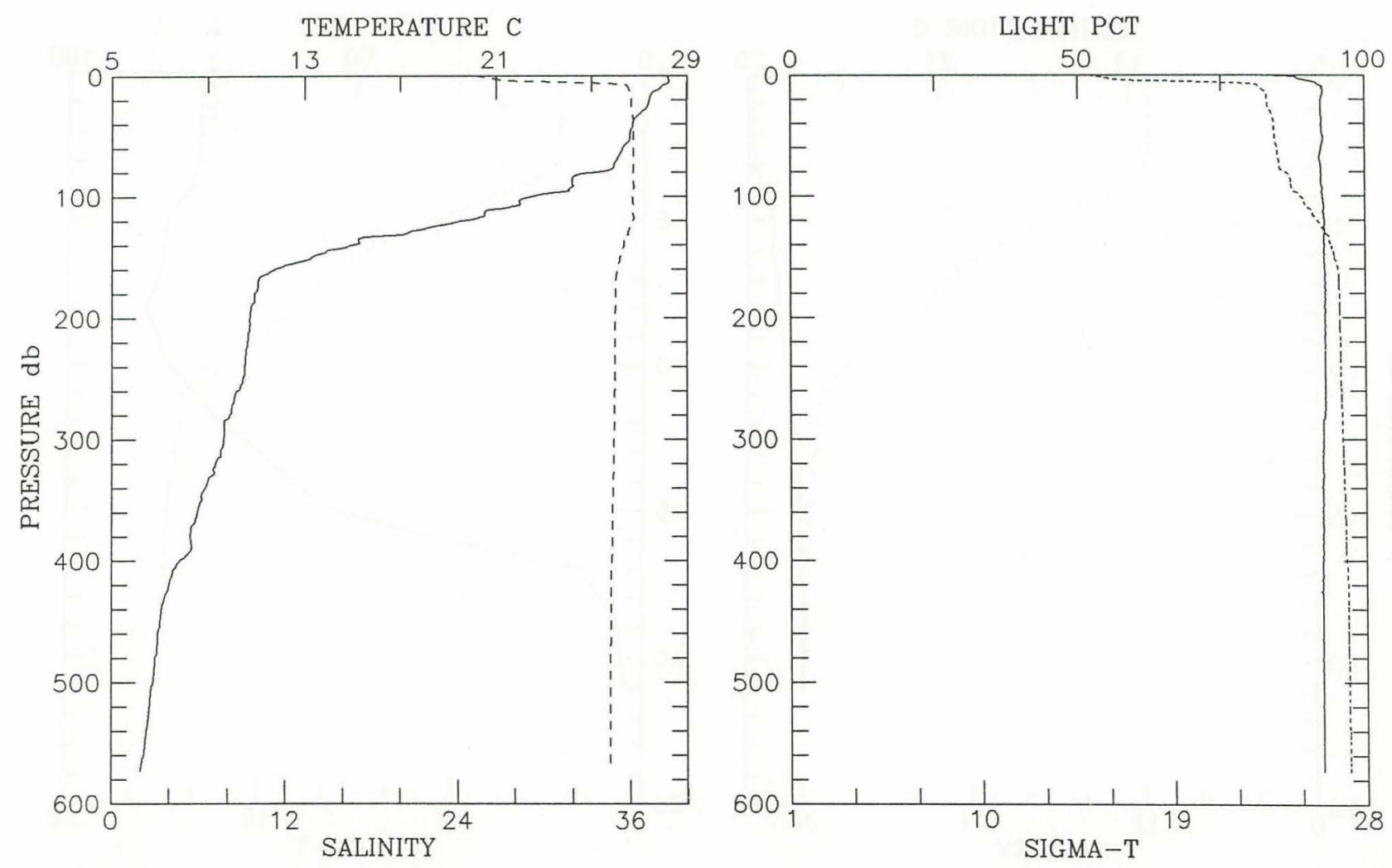


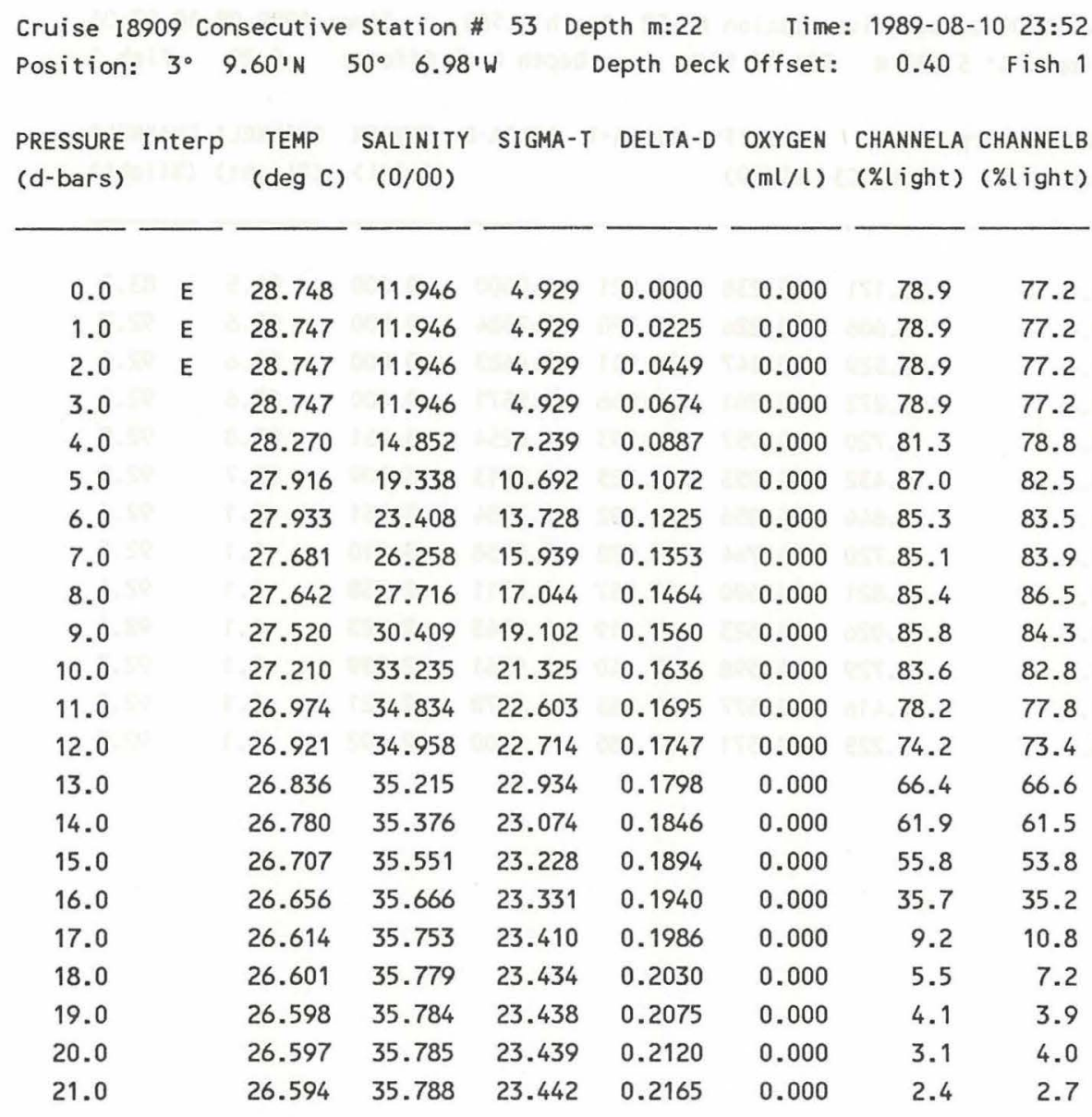
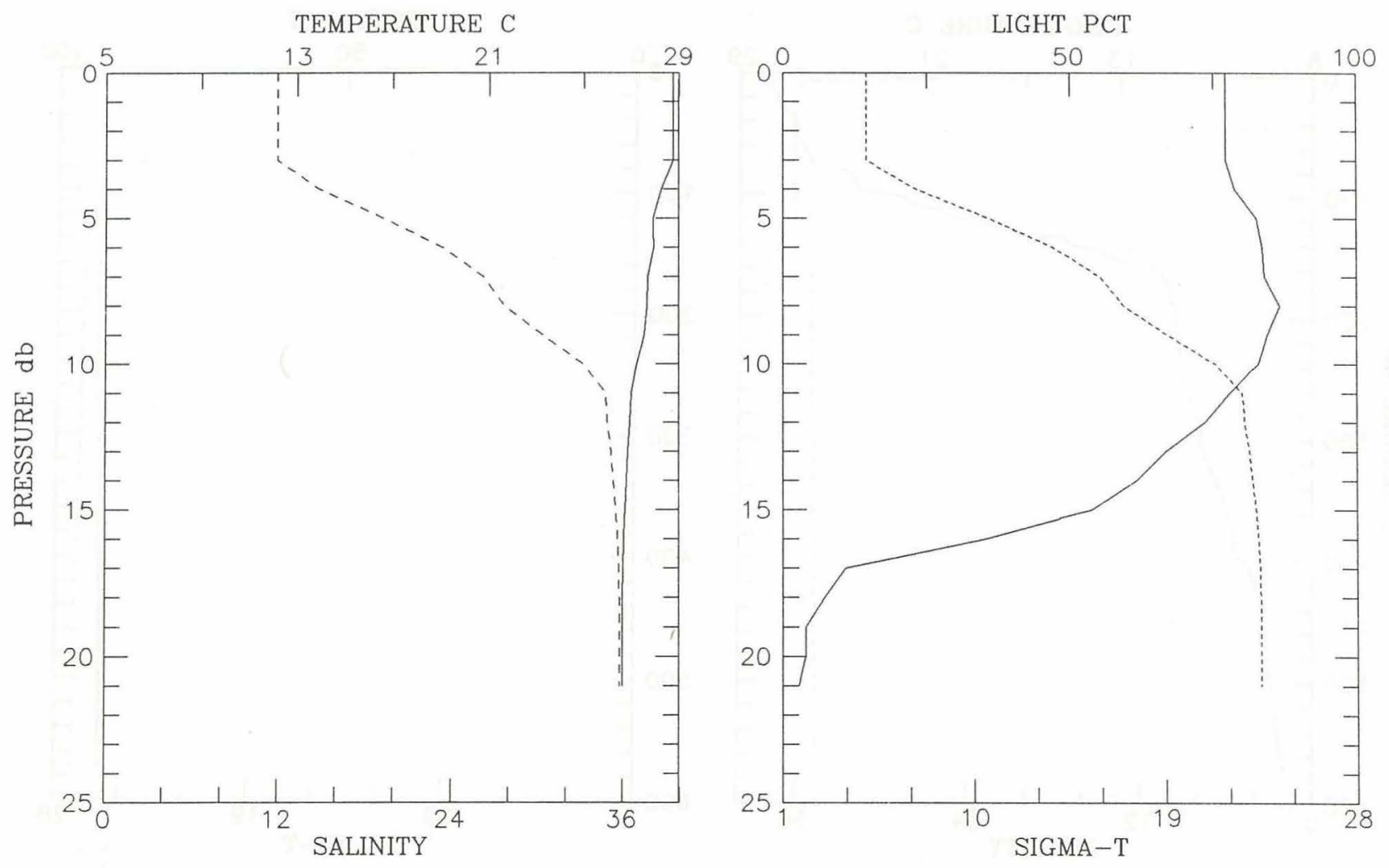


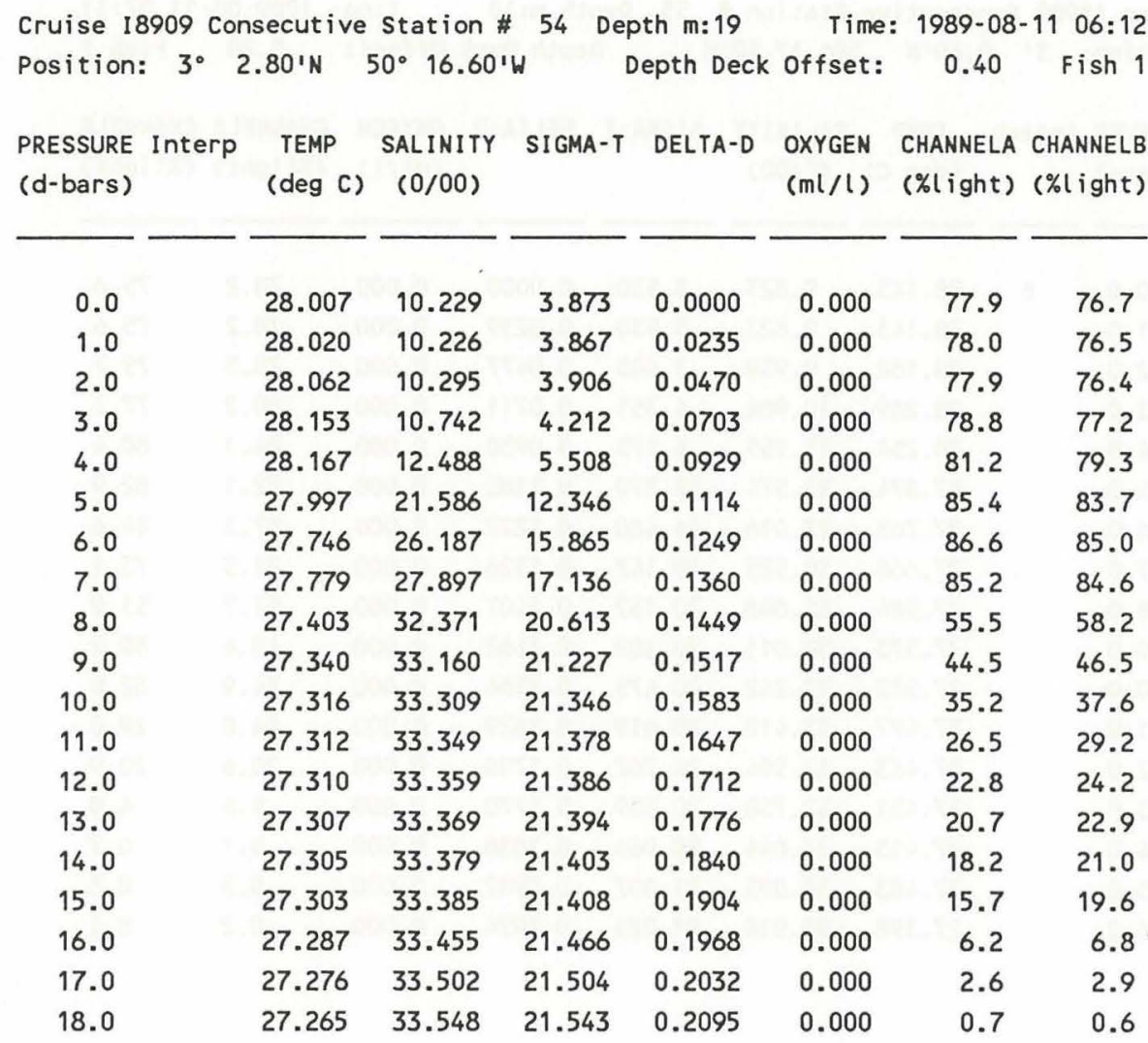
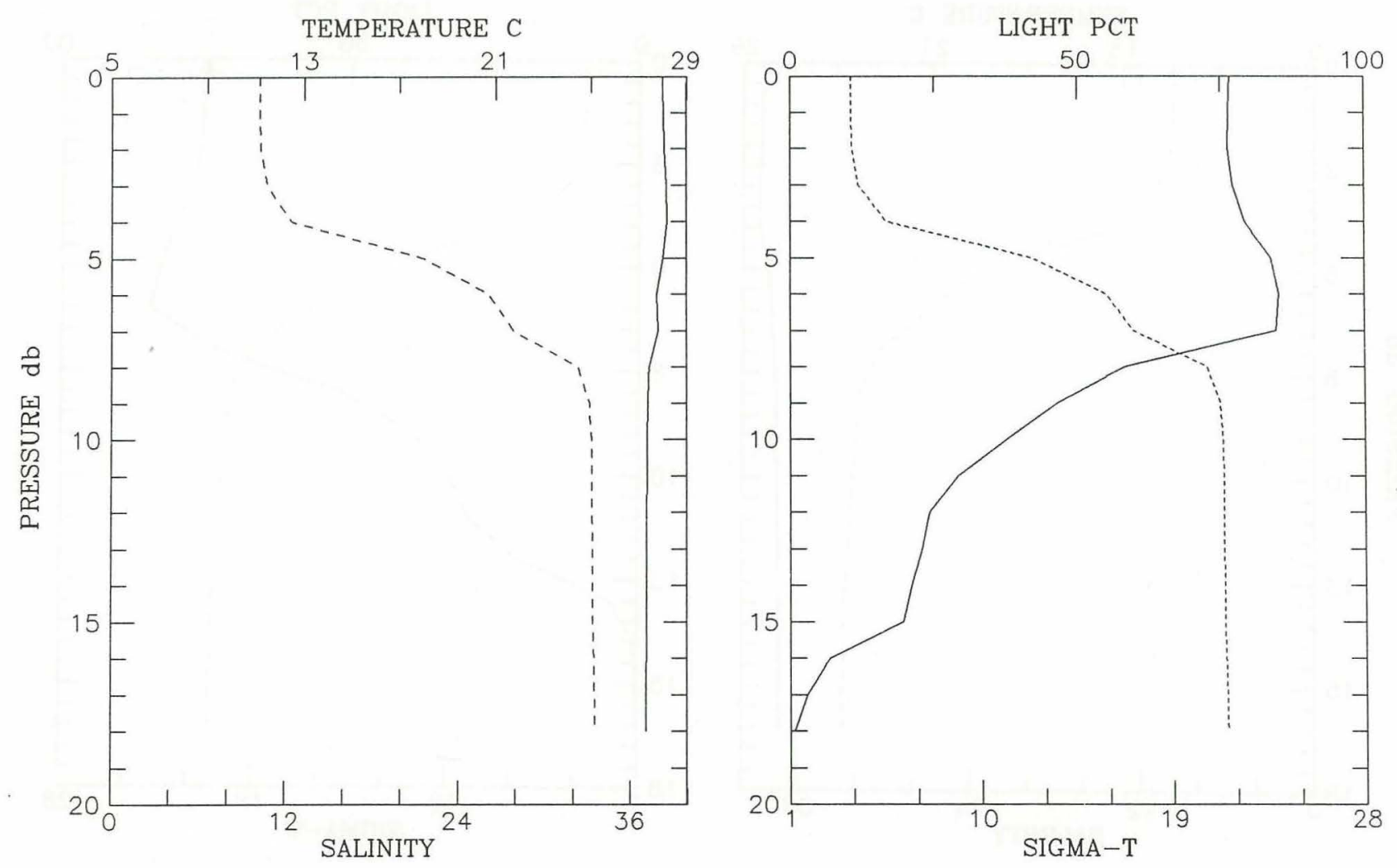


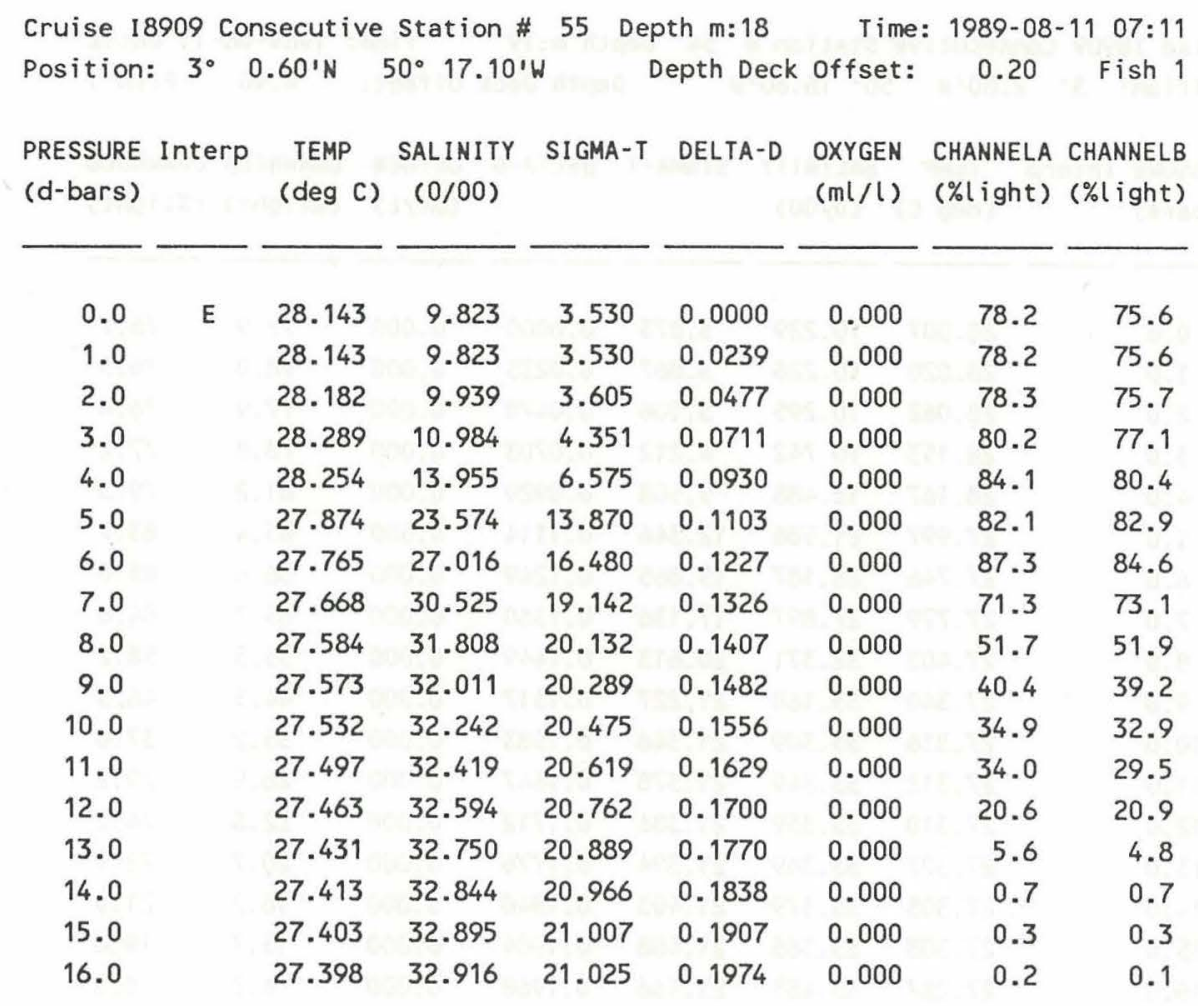
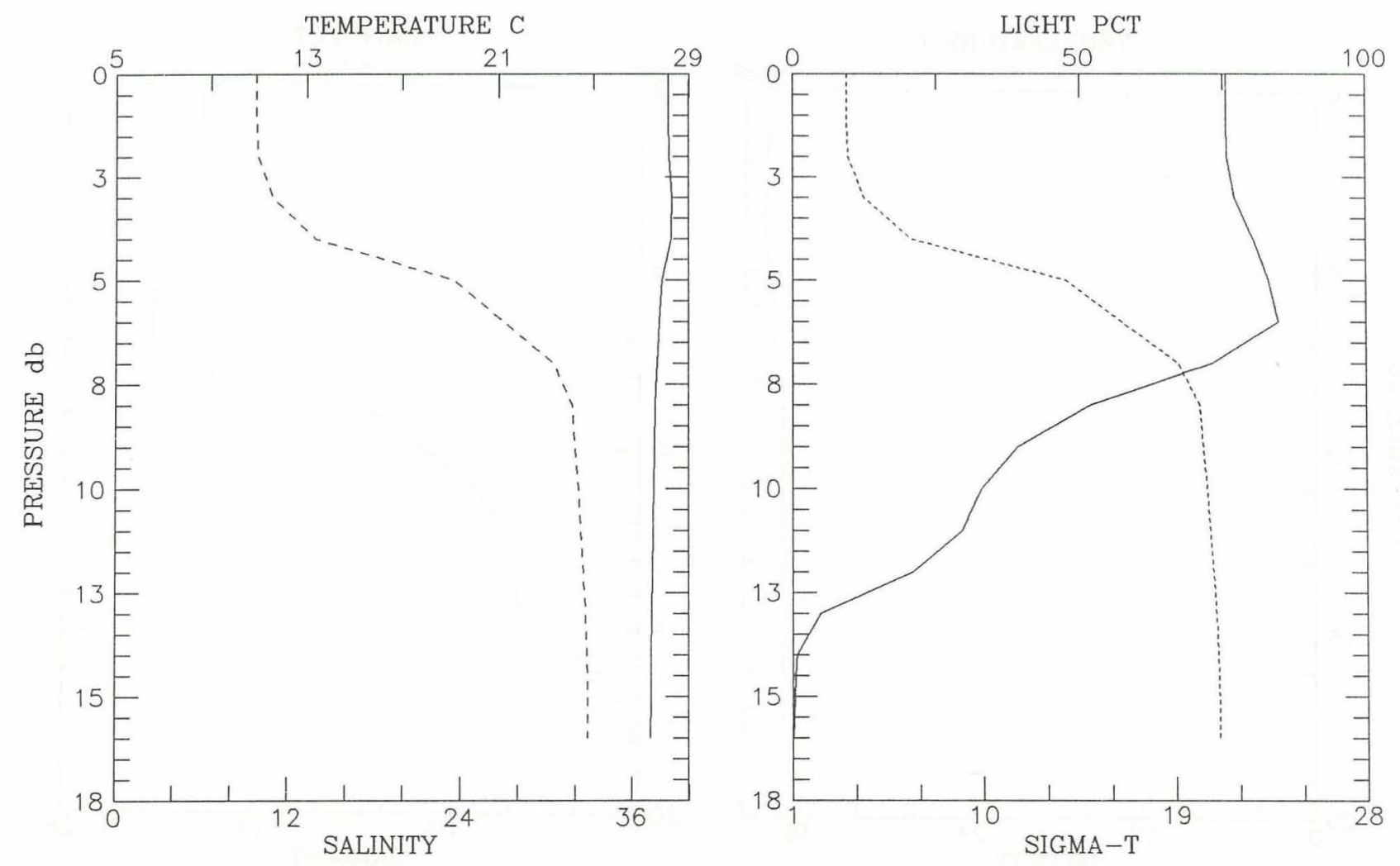


\begin{tabular}{|c|c|c|c|c|c|c|c|c|}
\hline \multicolumn{9}{|c|}{ Cruise 18909 Consecutive Station \# 56 Depth m:17 } \\
\hline Position: & $: 3^{\circ}$ & $3.50^{\prime} \mathrm{N}$ & $50^{\circ} 16.90^{\prime}$ & & epth Deck & offset: & 0.20 & Fish 1 \\
\hline $\begin{array}{l}\text { PRESSURE } \\
\text { (d-bars) }\end{array}$ & Interp & $\begin{array}{c}\text { TEMP } \\
(\operatorname{deg} C)\end{array}$ & $\begin{array}{l}\text { SALINITY } \\
(0 / 00)\end{array}$ & SIGMA-T & DELTA-D & $\begin{array}{l}\text { OXYGEN } \\
(m l / l)\end{array}$ & $\begin{array}{l}\text { CHANNELA } \\
\text { (\%light) }\end{array}$ & $\begin{array}{l}\text { CHANNELB } \\
\text { (\%light) }\end{array}$ \\
\hline 0.0 & E & 28.100 & 10.066 & 3.724 & 0.0000 & 0.000 & 80.0 & 77.9 \\
\hline 1.0 & & 28.099 & 10.066 & 3.724 & 0.0237 & 0.000 & 80.0 & 77.9 \\
\hline 2.0 & & 28.130 & 10.181 & 3.801 & 0.0473 & 0.000 & 79.9 & 77.8 \\
\hline 3.0 & & 28.201 & 10.665 & 4.140 & 0.0707 & 0.000 & 81.0 & 78.9 \\
\hline 4.0 & & 28.211 & 14.186 & 6.760 & 0.0926 & 0.000 & 83.8 & 81.6 \\
\hline 5.0 & & 27.981 & 21.934 & 12.611 & 0.1104 & 0.000 & 86.2 & 84.6 \\
\hline 6.0 & & 27.792 & 26.292 & 15.930 & 0.1237 & 0.000 & 86.8 & 84.4 \\
\hline 7.0 & & 27.797 & 27.988 & 17.199 & 0.1348 & 0.000 & 86.0 & 84.1 \\
\hline 8.0 & & 27.778 & 29.880 & 18.623 & 0.1446 & 0.000 & 75.5 & 74.4 \\
\hline 9.0 & & 27.768 & 30.479 & 19.076 & 0.1535 & 0.000 & 66.0 & 65.3 \\
\hline 10.0 & & 27.717 & 31.059 & 19.528 & 0.1619 & 0.000 & 50.0 & 50.8 \\
\hline 11.0 & & 27.661 & 31.473 & 19.856 & 0.1700 & 0.000 & 16.2 & 18.2 \\
\hline 12.0 & & 27.605 & 31.795 & 20.116 & 0.1777 & 0.000 & 2.0 & 2.8 \\
\hline 13.0 & & 27.576 & 31.957 & 20.247 & 0.1853 & 0.000 & 0.7 & 0.6 \\
\hline 14.0 & & 27.556 & 32.066 & 20.335 & 0.1928 & 0.000 & 0.3 & 0.4 \\
\hline 15.0 & & 27.539 & 32.150 & 20.404 & 0.2002 & 0.000 & 0.2 & 0.1 \\
\hline
\end{tabular}
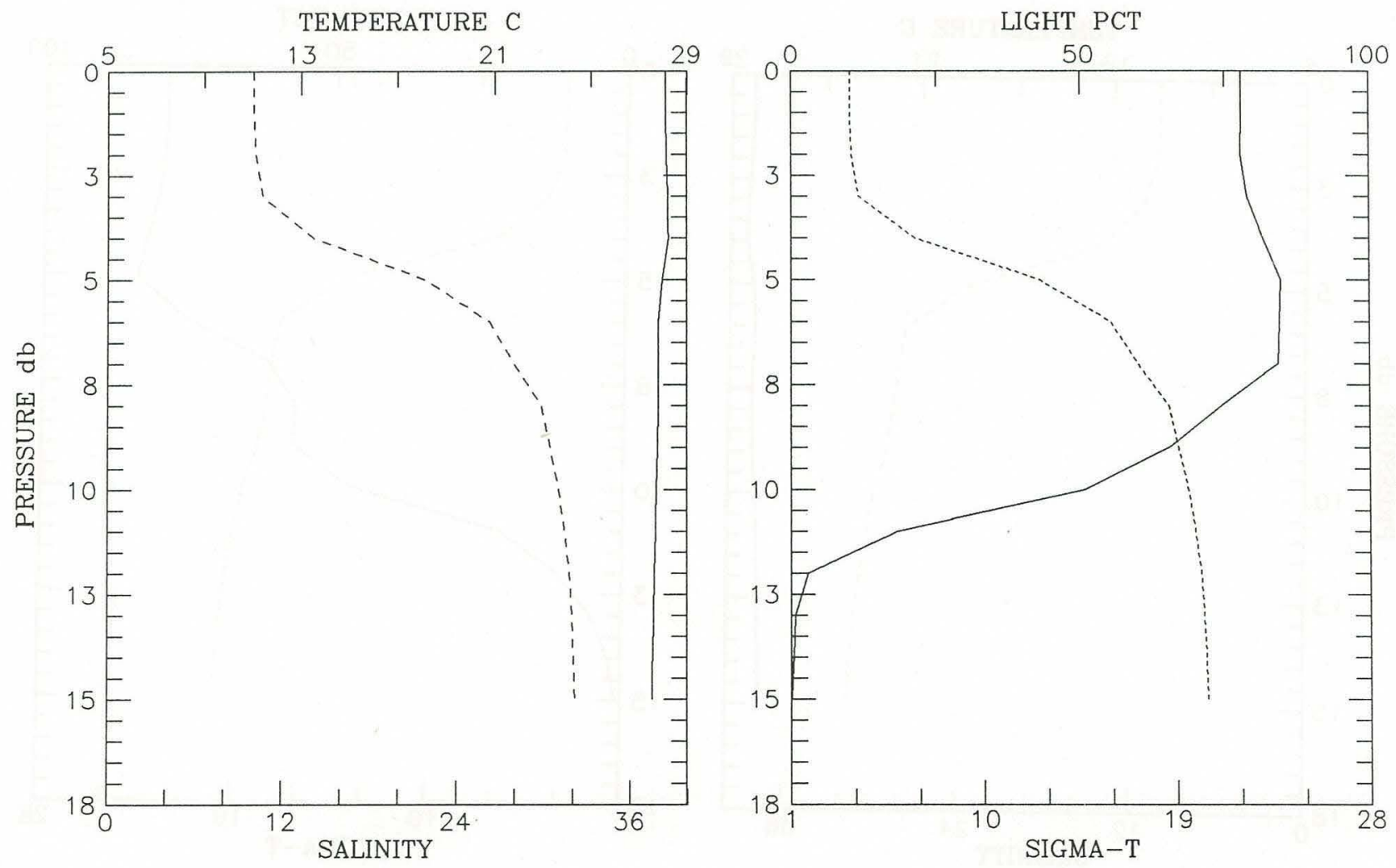


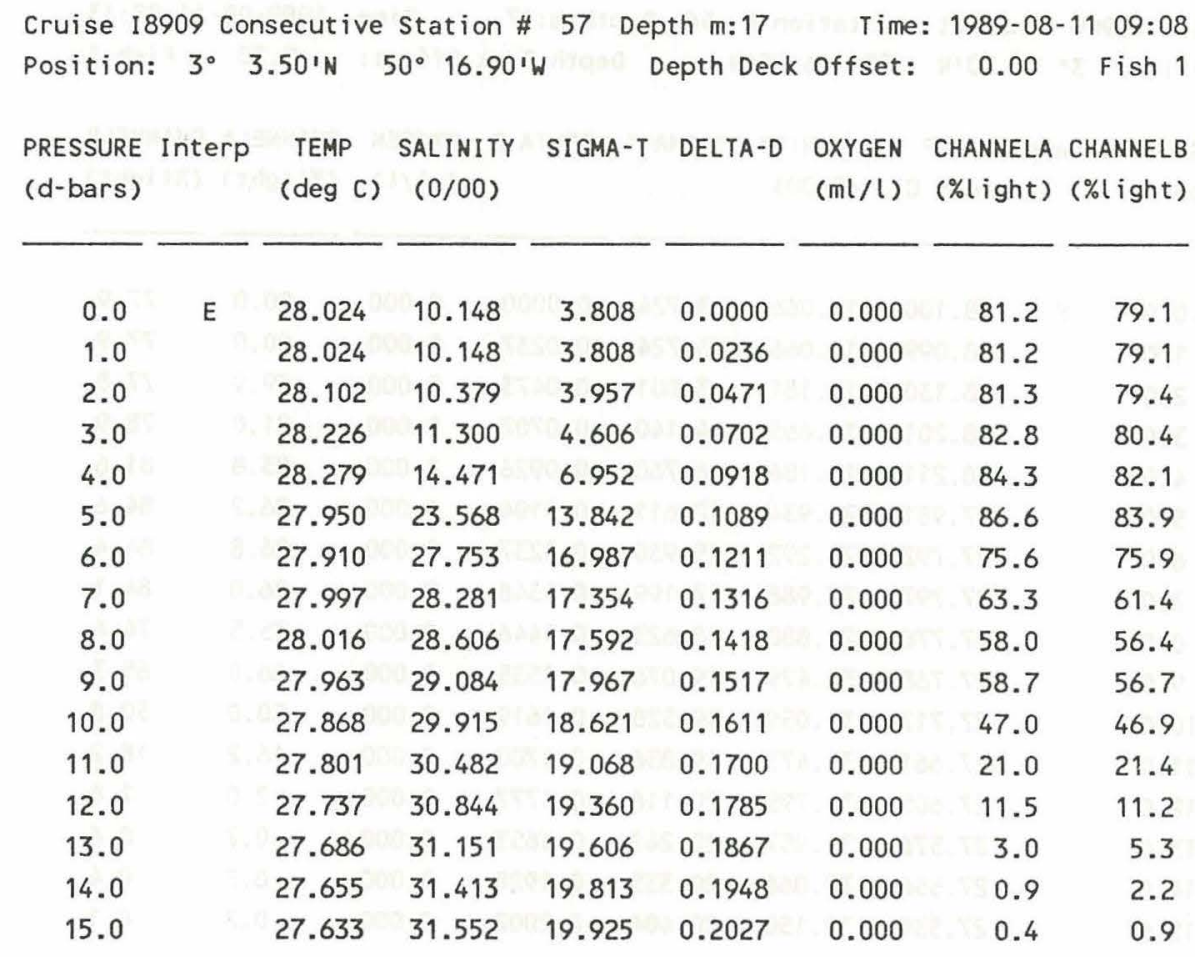
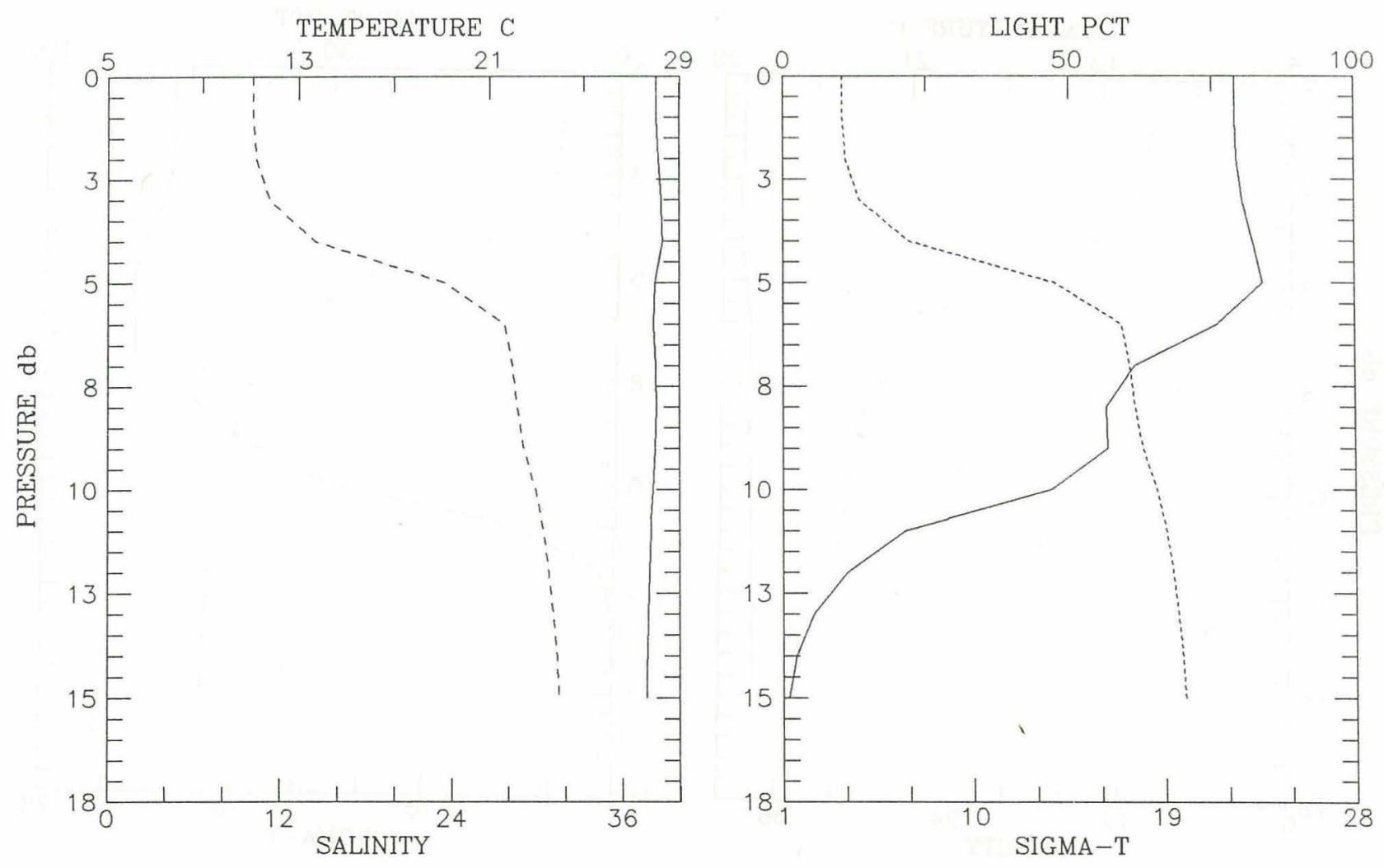


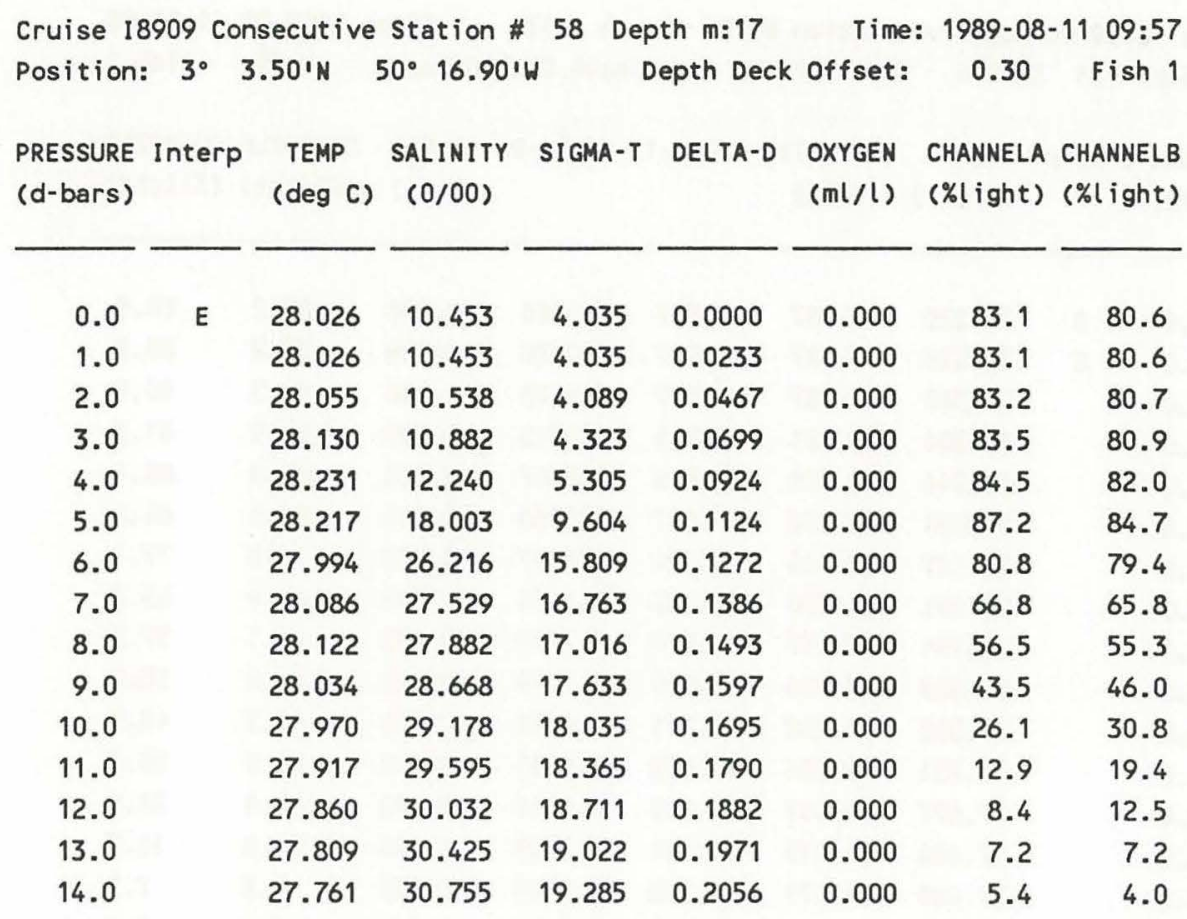
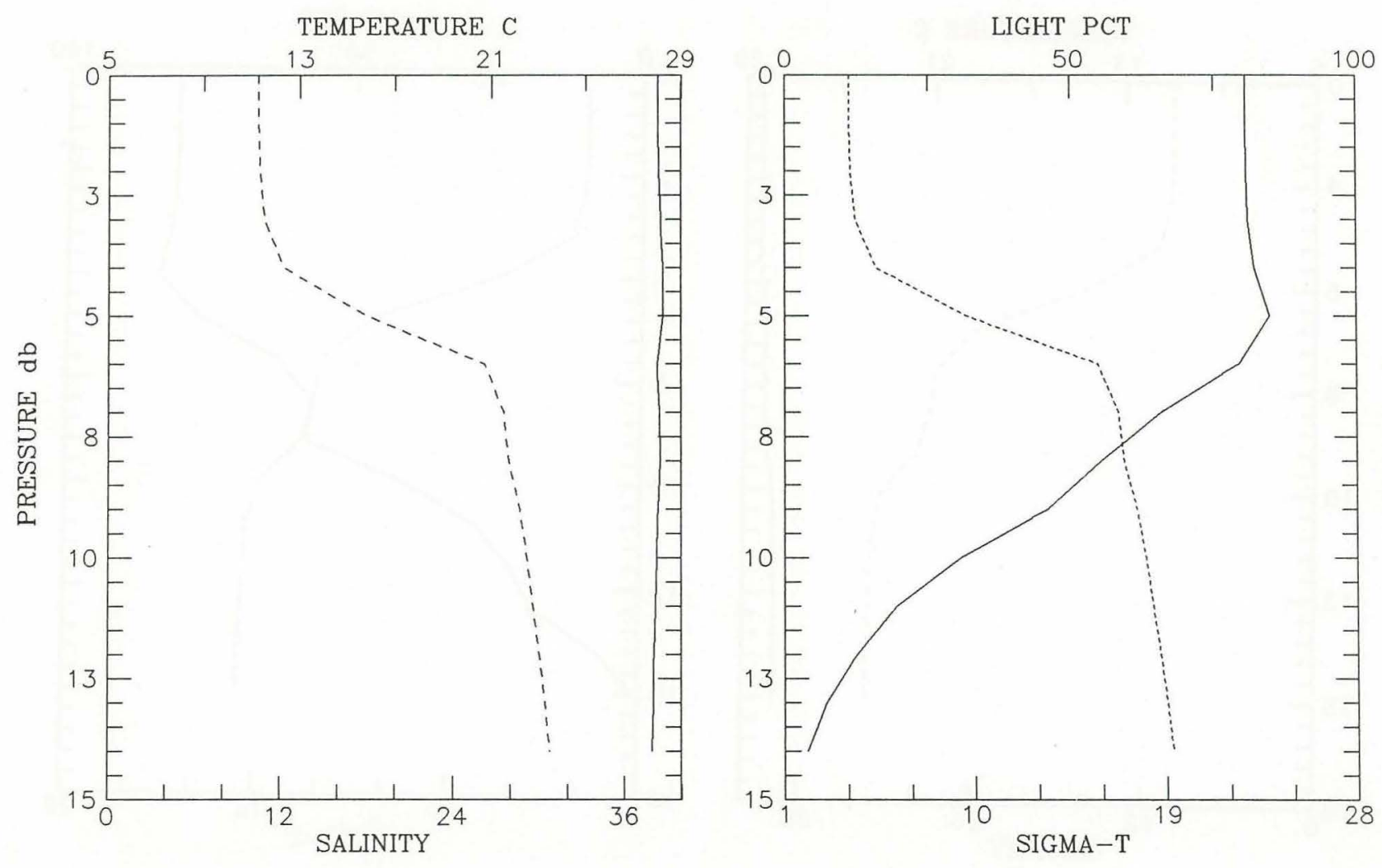


\begin{tabular}{|c|c|c|c|c|c|c|c|c|}
\hline \multirow{2}{*}{$\begin{array}{l}\text { Cruise I } \\
\text { Position } \\
\text { PRESSURE } \\
\text { (d-bars) }\end{array}$} & $\begin{array}{l}8909 \\
: \quad 3^{\circ}\end{array}$ & $\begin{array}{l}\text { nsecut ive } \\
3.501 \mathrm{~N}\end{array}$ & $\begin{array}{l}\text { e station \# } \\
50^{\circ} 16.90^{\prime} \mathrm{W}\end{array}$ & $\begin{array}{r}59 \text { Def } \\
W\end{array}$ & $\begin{array}{l}\text { th } \mathrm{m:} 17 \\
\text { epth Deck }\end{array}$ & Time: & $\begin{array}{c}1989-08- \\
0.40\end{array}$ & $\begin{array}{r}11 \text { 11:08 } \\
\text { Fish } 1\end{array}$ \\
\hline & Interp & $\begin{array}{l}\text { TEMP } \\
(\operatorname{deg} C)\end{array}$ & $\begin{array}{l}\text { SALINITY } \\
(0 / 00)\end{array}$ & SIGMA-T & DELTA-D & $\begin{array}{l}\text { OXYGEN } \\
(m l / l)\end{array}$ & $\begin{array}{l}\text { CHANNELA } \\
\text { (\%light) }\end{array}$ & $\begin{array}{l}\text { CHANNELB } \\
\text { (\%light) }\end{array}$ \\
\hline 0.0 & $E$ & 28.329 & 9.987 & 3.597 & 0.0000 & 0.000 & 83.2 & 80.9 \\
\hline 1.0 & $E$ & 28.329 & 9.987 & 3.597 & 0.0238 & 0.000 & 83.2 & 80.9 \\
\hline 2.0 & & 28.329 & 9.987 & 3.597 & 0.0476 & 0.000 & 83.2 & 80.9 \\
\hline 3.0 & & 28.344 & 10.191 & 3.745 & 0.0713 & 0.000 & 83.9 & 81.3 \\
\hline 4.0 & & 28.344 & 10.901 & 4.273 & 0.0947 & 0.000 & 84.8 & 82.1 \\
\hline 5.0 & & 28.264 & 15.706 & 7.877 & 0.1160 & 0.000 & 85.5 & 84.0 \\
\hline 6.0 & & 28.127 & 23.444 & 13.694 & 0.1327 & 0.000 & 79.0 & 77.1 \\
\hline 7.0 & & 28.191 & 26.555 & 16.000 & 0.1454 & 0.000 & 63.9 & 63.2 \\
\hline 8.0 & & 28.151 & 27.017 & 16.359 & 0.1569 & 0.000 & 61.1 & 57.3 \\
\hline 9.0 & & 28.068 & 27.756 & 16.939 & 0.1679 & 0.000 & 59.8 & 58.0 \\
\hline 10.0 & & 27.822 & 30.362 & 18.971 & 0.1776 & 0.000 & 41.3 & 40.8 \\
\hline 11.0 & & 27.731 & 30.964 & 19.452 & 0.1861 & 0.000 & 29.5 & 28.7 \\
\hline 12.0 & & 27.697 & 31.147 & 19.600 & 0.1944 & 0.000 & 27.0 & 22.5 \\
\hline 13.0 & & 27.686 & 31.215 & 19.654 & 0.2025 & 0.000 & 20.8 & 18.7 \\
\hline 14.0 & & 27.666 & 31.371 & 19.778 & 0.2105 & 0.000 & 4.3 & 7.5 \\
\hline 15.0 & & 27.658 & 31.406 & 19.807 & 0.2185 & 0.000 & 1.0 & 1.0 \\
\hline
\end{tabular}
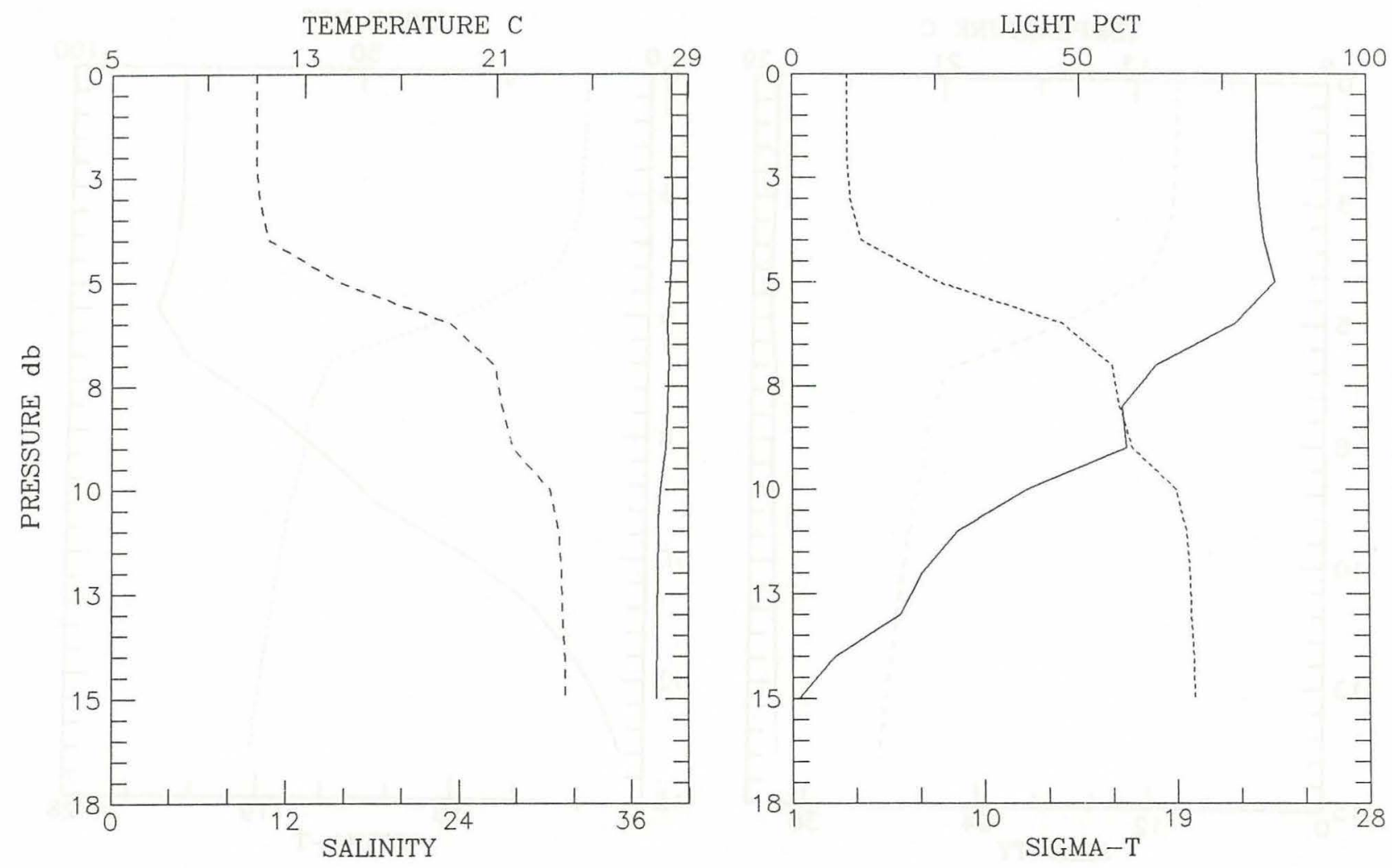


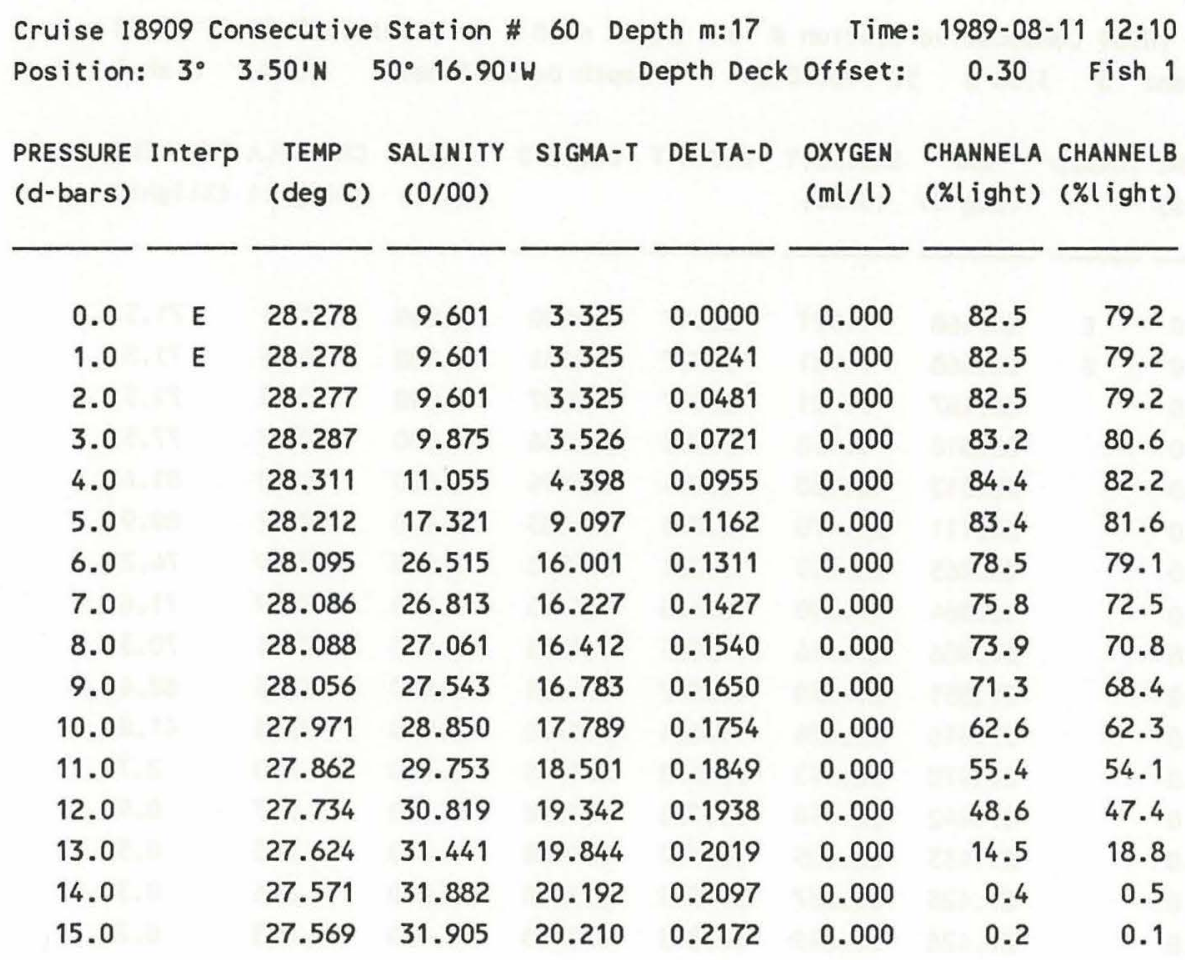
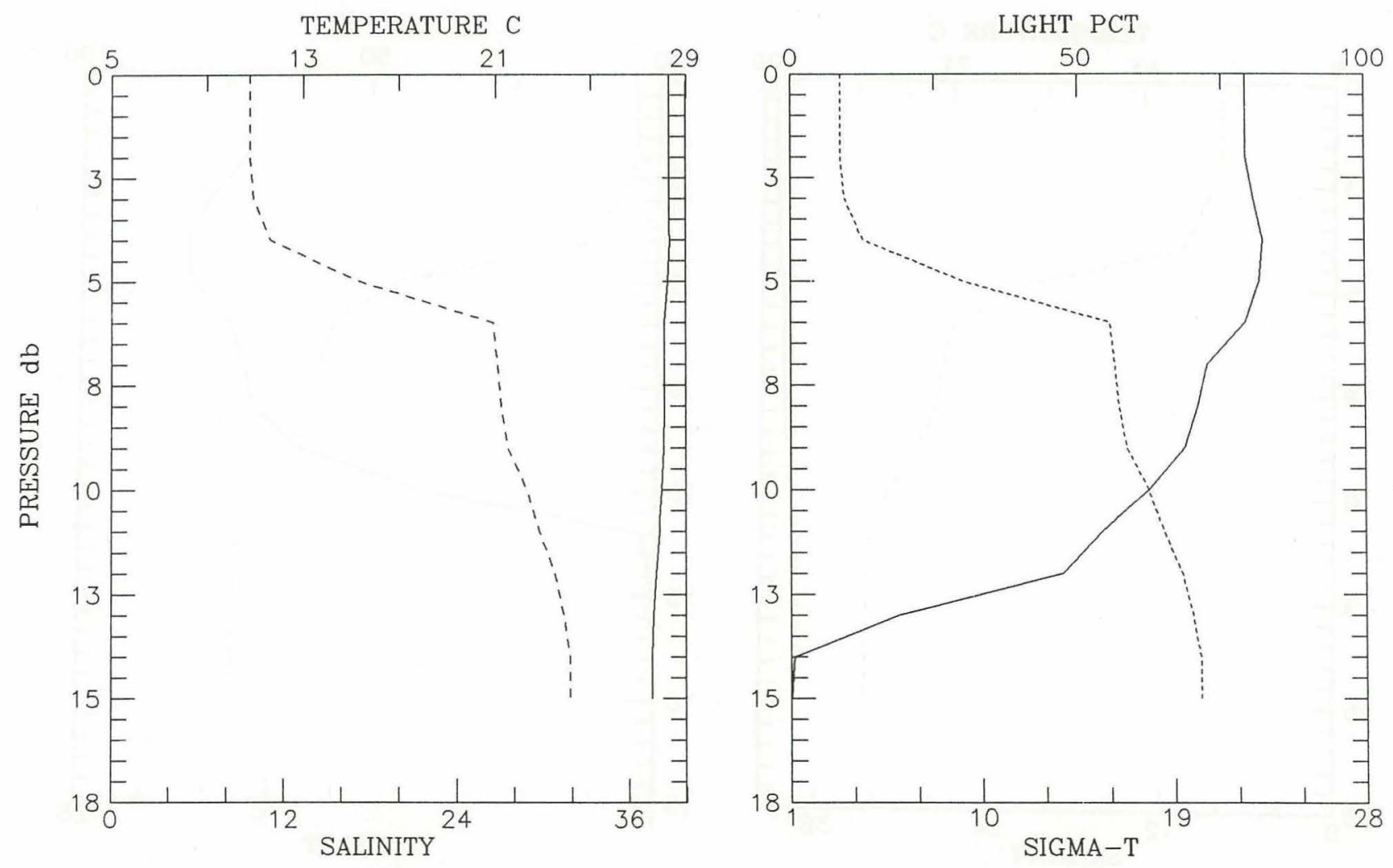


\begin{tabular}{|c|c|c|c|c|c|c|c|c|}
\hline Position: & $3^{\circ} 3$ & $.50^{\prime} \mathrm{N}$ & $50^{\circ} 16.90^{\prime}$ & & epth Deck & Offset: & 0.50 & Fish 1 \\
\hline $\begin{array}{l}\text { PRESSURE } \\
\text { (d-bars) }\end{array}$ & Interp & $\begin{array}{l}\text { TEMP } \\
(\operatorname{deg} C)\end{array}$ & $\begin{array}{l}\text { SALINITY } \\
(0 / 00)\end{array}$ & SIGMA-T & DELTA-D & $\begin{array}{l}\text { OXYGEN } \\
(\mathrm{ml} / \mathrm{l})\end{array}$ & $\begin{array}{l}\text { CHANNELA } \\
(\% \text { light) }\end{array}$ & $\begin{array}{l}\text { CHANNELB } \\
(\% \text { ight })\end{array}$ \\
\hline 0.0 & $E$ & 28.468 & 7.921 & 2.017 & 0.0000 & 0.000 & 74.5 & 71.5 \\
\hline 1.0 & $E$ & 28.468 & 7.921 & 2.017 & 0.0254 & 0.000 & 74.5 & 71.5 \\
\hline 2.0 & & 28.467 & 7.921 & 2.017 & 0.0507 & 0.000 & 74.5 & 71.5 \\
\hline 3.0 & & 28.318 & 9.158 & 2.983 & 0.0756 & 0.000 & 79.7 & 77.5 \\
\hline 4.0 & & 28.312 & 10.660 & 4.104 & 0.0994 & 0.000 & 84.0 & 81.4 \\
\hline 5.0 & & 28.111 & 22.470 & 12.971 & 0.1183 & 0.000 & 83.2 & 80.9 \\
\hline 6.0 & & 28.065 & 26.809 & 16.231 & 0.1313 & 0.000 & 75.9 & 74.2 \\
\hline 7.0 & & 28.064 & 27.390 & 16.666 & 0.1425 & 0.000 & 72.9 & 71.6 \\
\hline 8.0 & & 27.986 & 27.916 & 17.085 & 0.1533 & 0.000 & 71.1 & 70.3 \\
\hline 9.0 & & 27.851 & 29.530 & 18.337 & 0.1633 & 0.000 & 63.5 & 62.4 \\
\hline 10.0 & & 27.616 & 31.224 & 19.684 & 0.1720 & 0.000 & 33.3 & 41.0 \\
\hline 11.0 & & 27.470 & 32.353 & 20.578 & 0.1796 & 0.000 & 2.0 & 2.7 \\
\hline 12.0 & & 27.442 & 32.550 & 20.736 & 0.1868 & 0.000 & 0.7 & 0.9 \\
\hline 13.0 & & 27.433 & 32.605 & 20.780 & 0.1938 & 0.000 & 0.5 & 0.5 \\
\hline 14.0 & & 27.428 & 32.637 & 20.805 & 0.2008 & 0.000 & 0.4 & 0.3 \\
\hline 15.0 & & 27.426 & 32.649 & 20.815 & 0.2078 & 0.000 & 0.3 & 0.2 \\
\hline
\end{tabular}
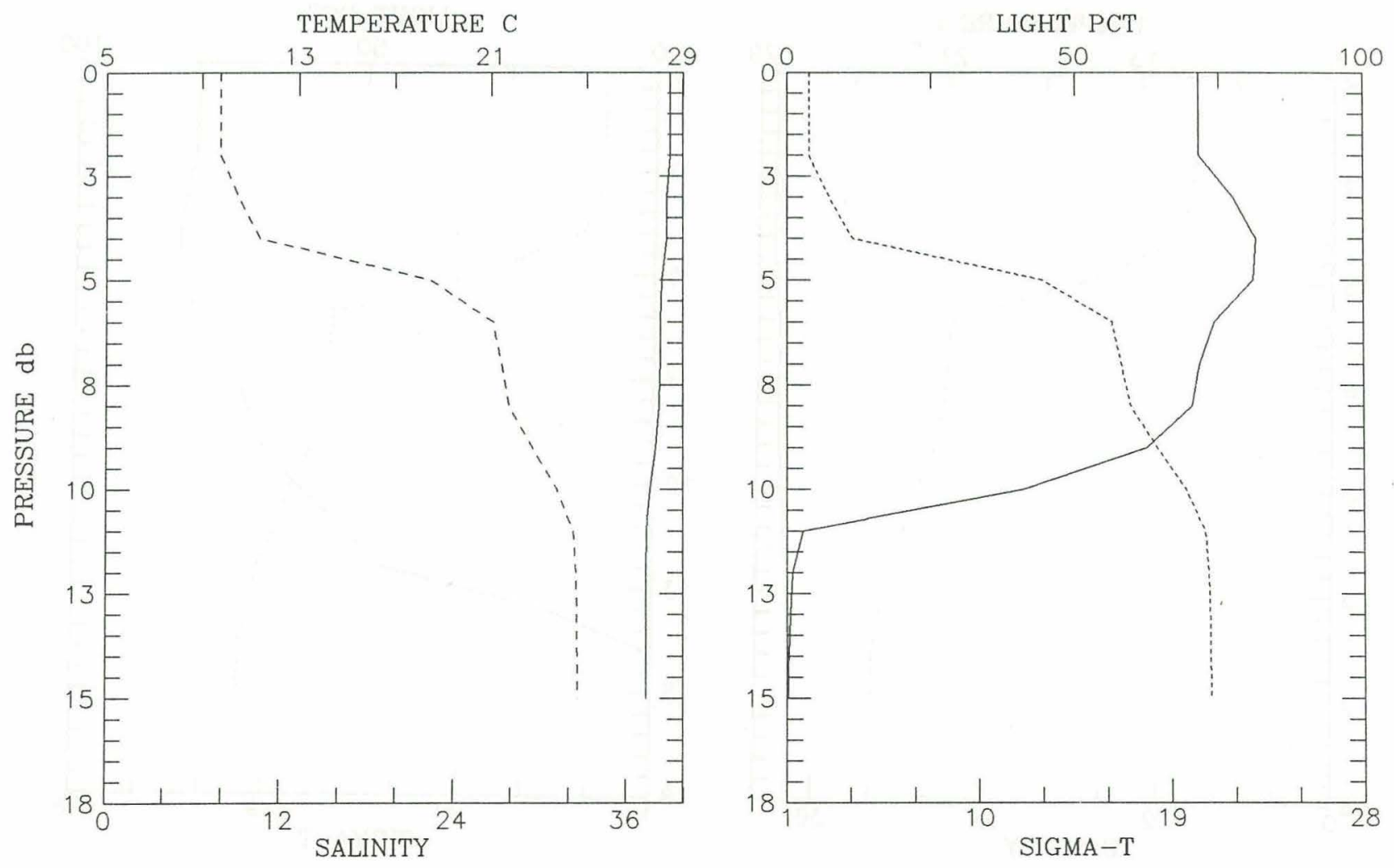


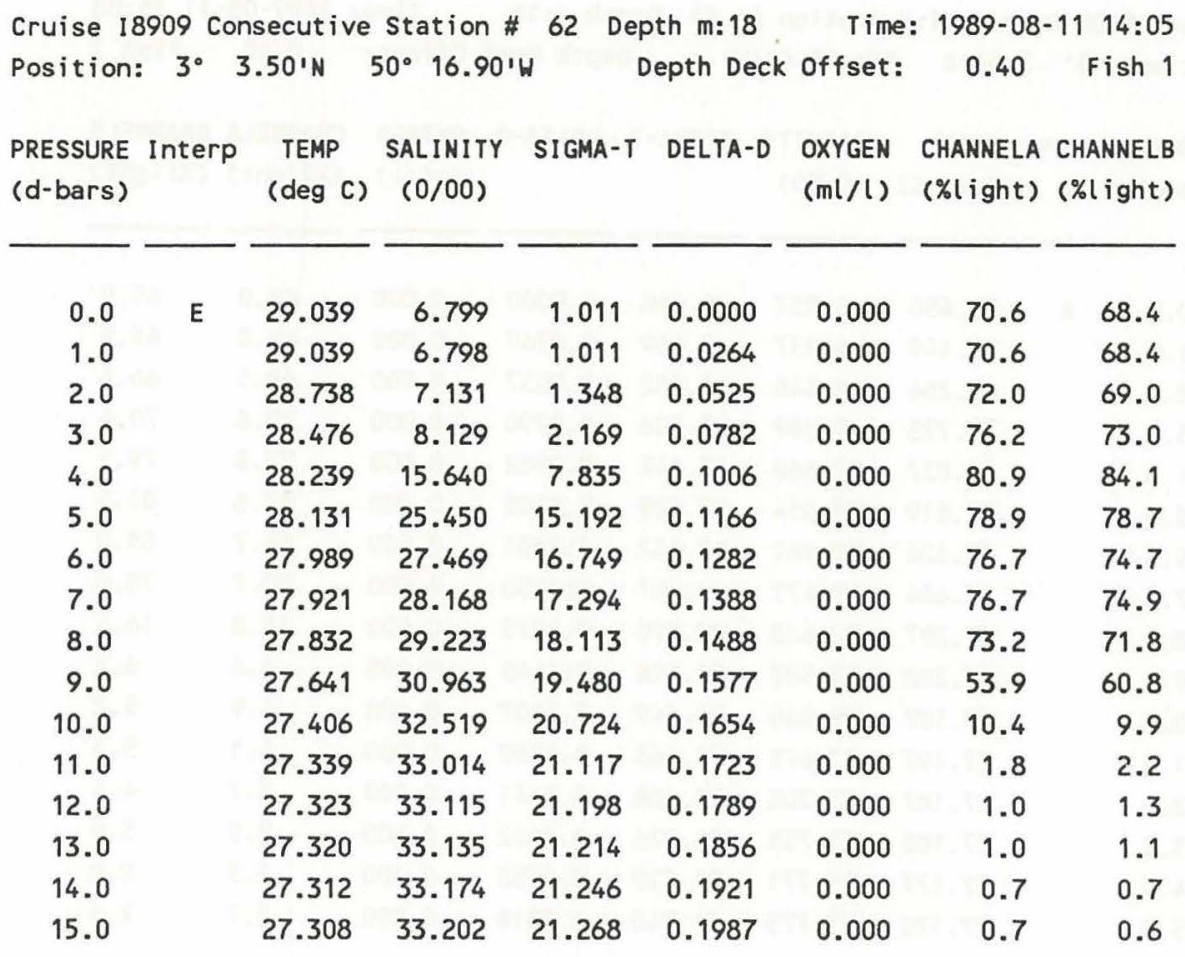
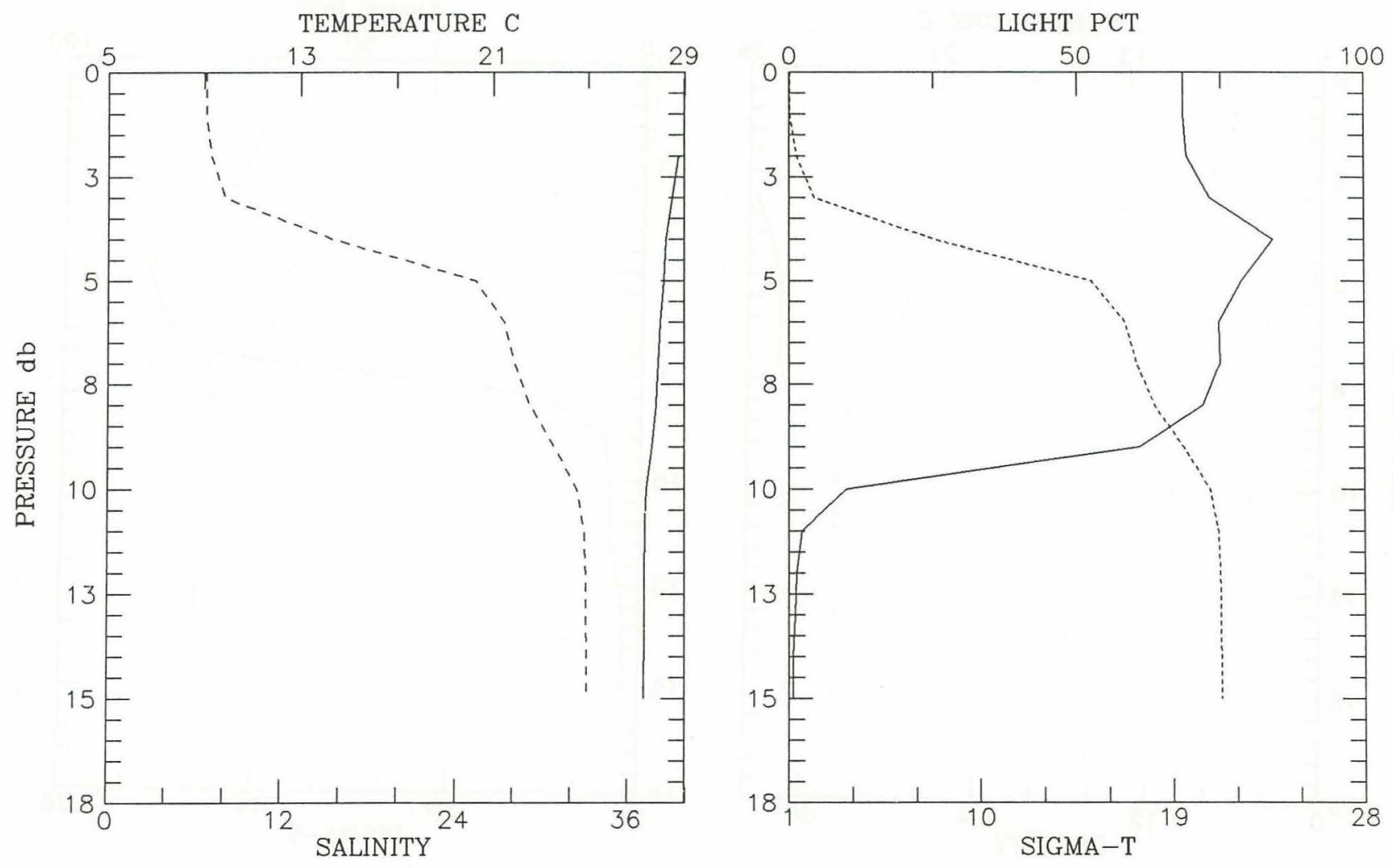


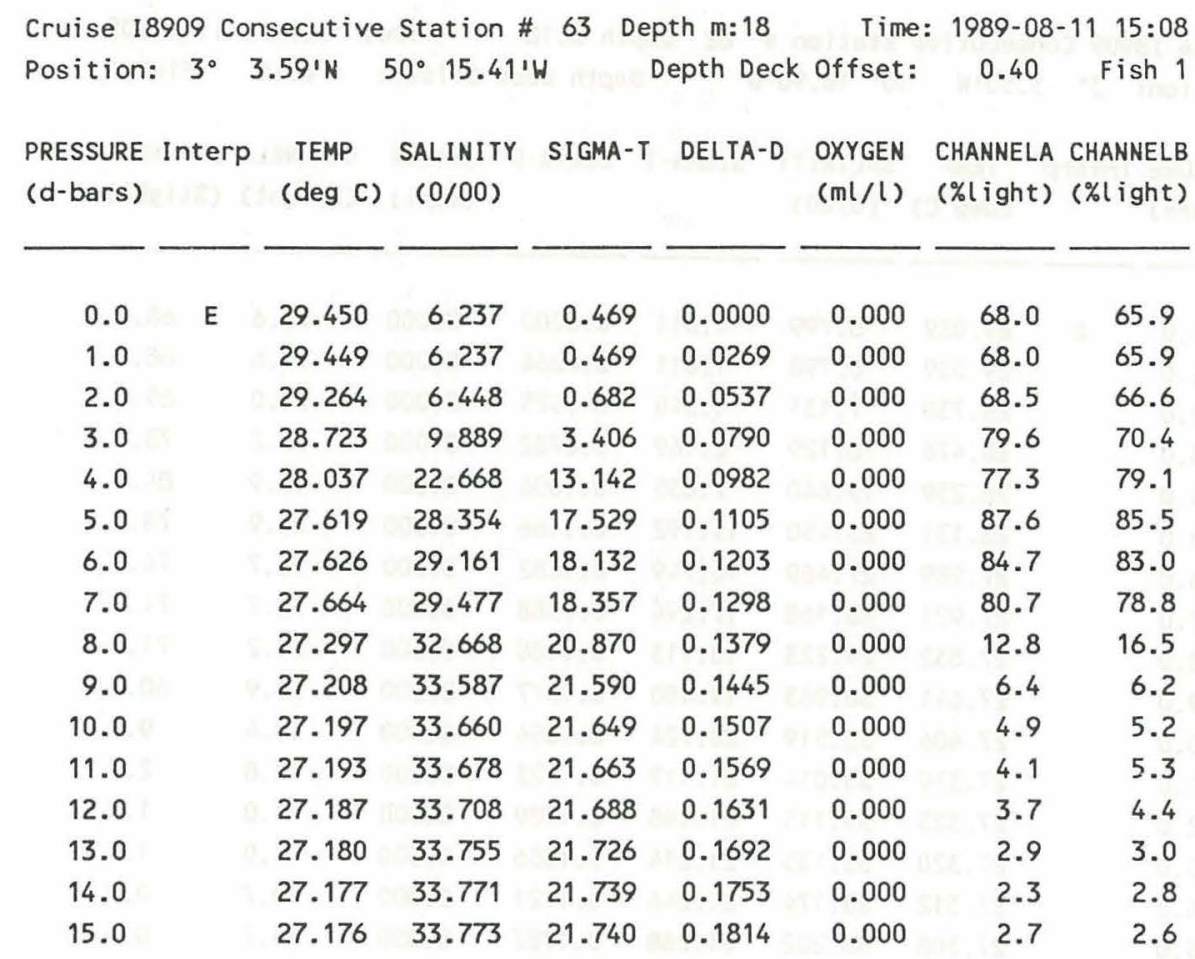
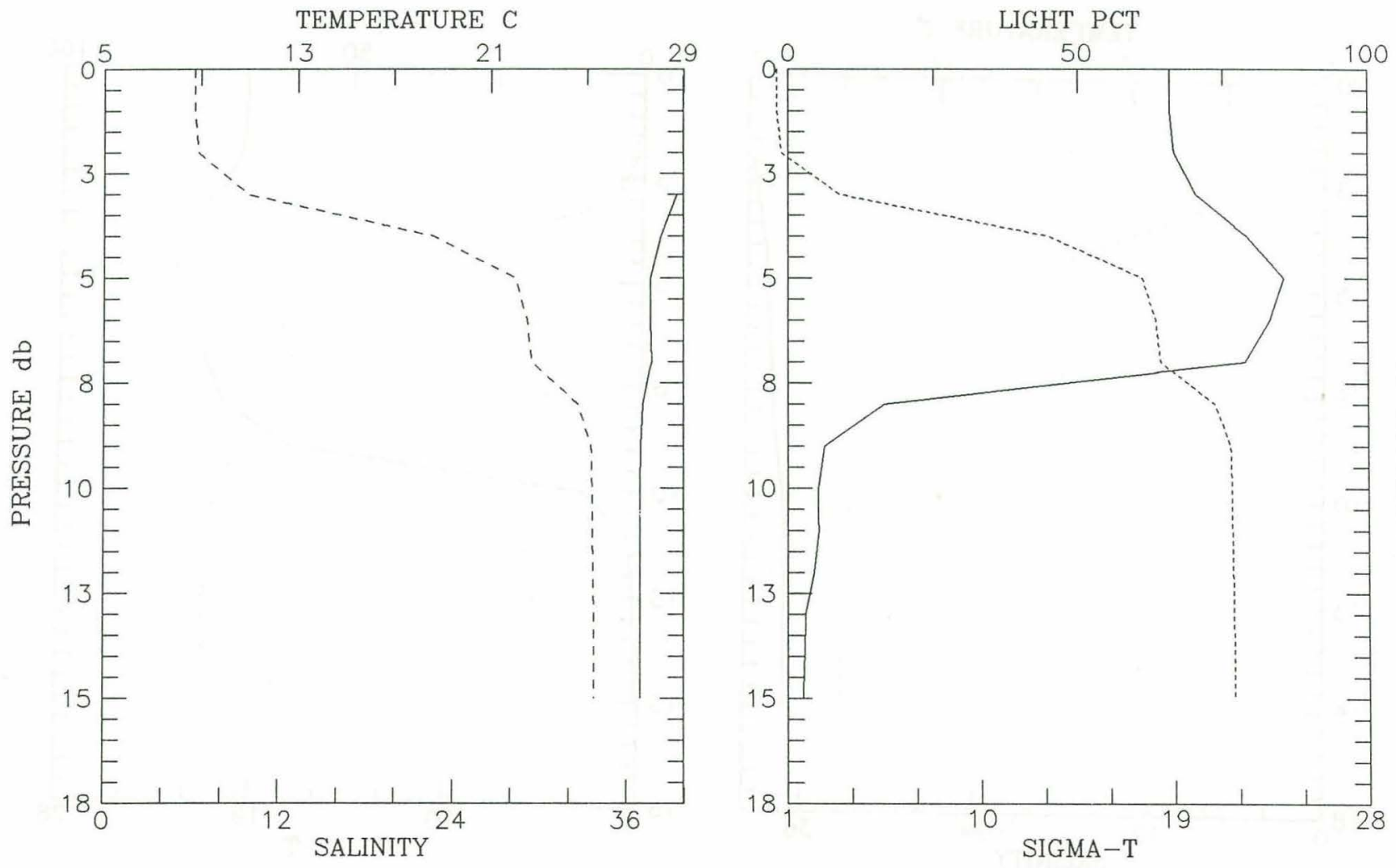


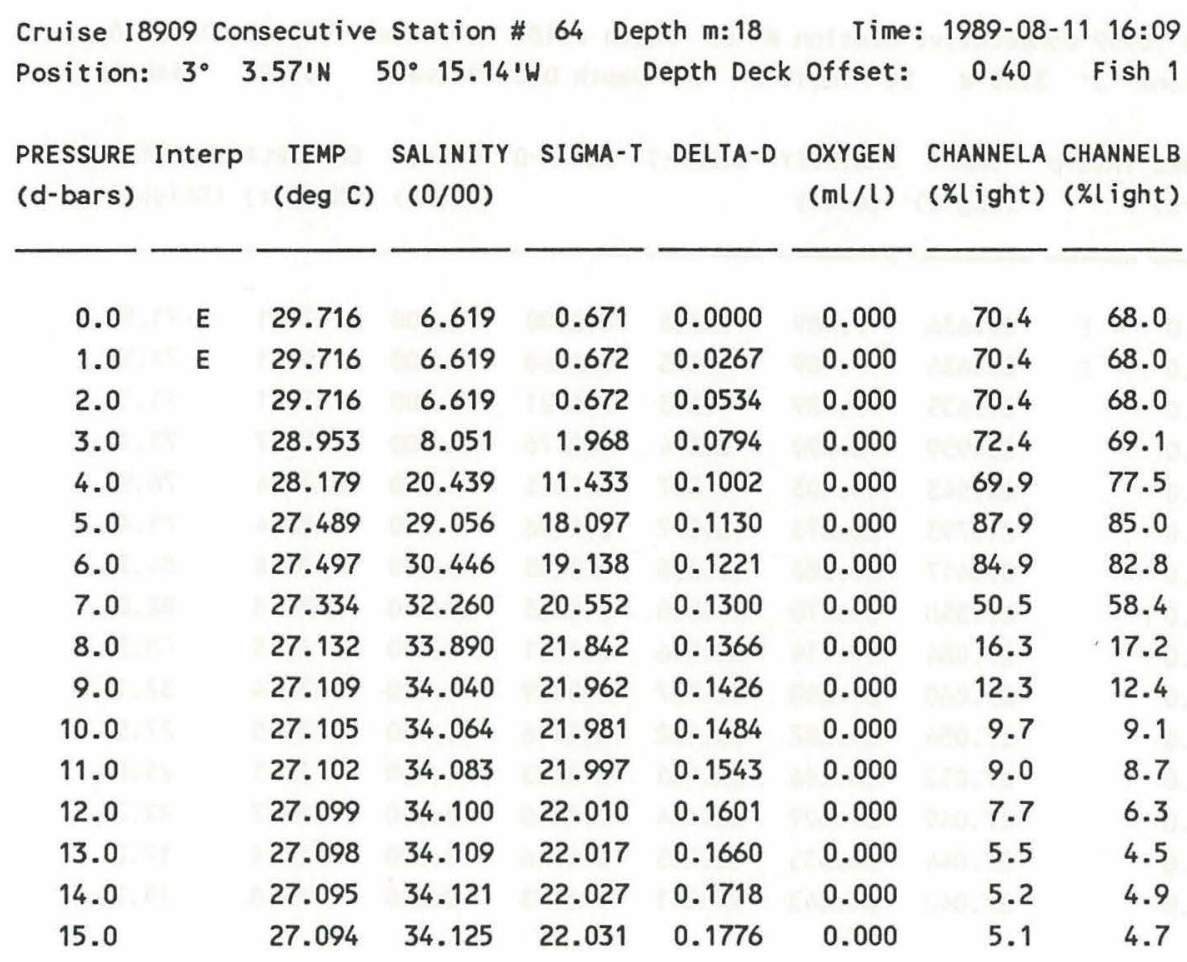
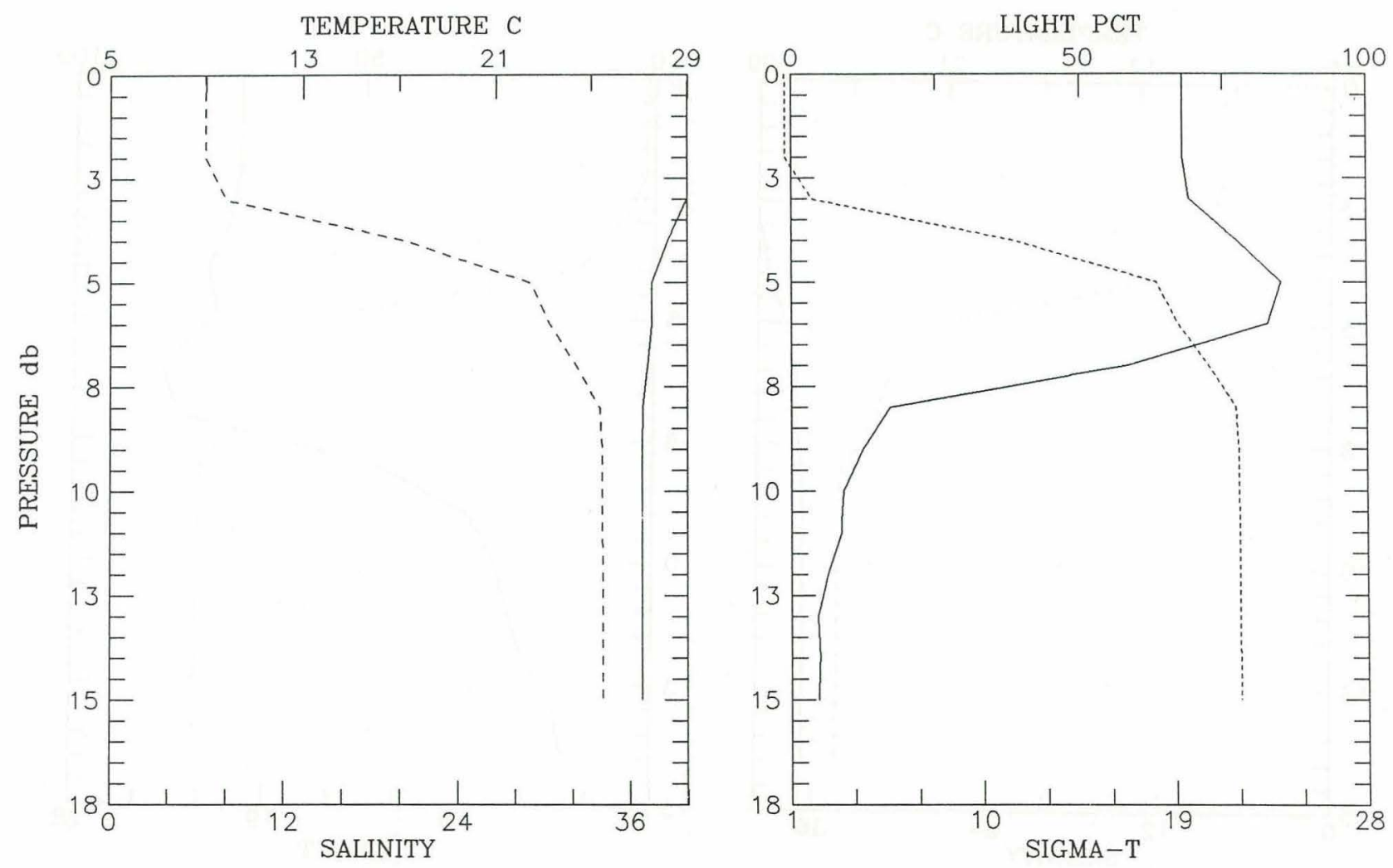


\begin{tabular}{|c|c|c|c|c|c|c|c|c|}
\hline $\begin{array}{l}\text { Cruise I8 } \\
\text { Position: }\end{array}$ & $\begin{array}{l}39090 \\
3^{\circ}\end{array}$ & 50 N & $50^{\circ} 16.19$ & 65 & epth Deck & offset: & 0.50 & Fish 1 \\
\hline $\begin{array}{l}\text { PRESSURE } \\
\text { (d-bars) }\end{array}$ & Interp & $\begin{array}{c}\text { TEMP } \\
(\operatorname{deg} \mathrm{C})\end{array}$ & $\begin{array}{l}\text { SALINITY } \\
(0 / 00)\end{array}$ & SIGMA-T & DELTA-D & $\begin{array}{l}\text { OXYGEN } \\
(\mathrm{ml} / \mathrm{l})\end{array}$ & $\begin{array}{l}\text { CHANNELA } \\
\text { (\%light) }\end{array}$ & $\begin{array}{l}\text { CHANNELB } \\
\text { (\%light) }\end{array}$ \\
\hline 0.0 & $E$ & 29.636 & 7.489 & 1.343 & 0.0000 & 0.000 & 74.1 & 71.5 \\
\hline 1.0 & $E$ & 29.636 & 7.489 & 1.343 & 0.0260 & 0.000 & 74.1 & 71.5 \\
\hline 2.0 & & 29.635 & 7.489 & 1.343 & 0.0521 & 0.000 & 74.1 & 71.5 \\
\hline 3.0 & & 28.939 & 8.590 & 2.374 & 0.0776 & 0.000 & 76.7 & 73.1 \\
\hline 4.0 & & 28.543 & 11.403 & 4.587 & 0.1015 & 0.000 & 77.4 & 76.9 \\
\hline 5.0 & & 27.793 & 26.676 & 16.217 & 0.1186 & 0.000 & 86.4 & 75.4 \\
\hline 6.0 & & 27.417 & 30.586 & 19.268 & 0.1285 & 0.000 & 88.0 & 84.7 \\
\hline 7.0 & & 27.358 & 31.970 & 20.326 & 0.1365 & 0.000 & 80.3 & 82.0 \\
\hline 8.0 & & 27.084 & 34.114 & 22.026 & 0.1431 & 0.000 & 45.5 & 48.3 \\
\hline 9.0 & & 27.060 & 34.238 & 22.127 & 0.1489 & 0.000 & 34.4 & 32.1 \\
\hline 10.0 & & 27.054 & 34.282 & 22.162 & 0.1546 & 0.000 & 30.5 & 27.5 \\
\hline 11.0 & & 27.052 & 34.286 & 22.165 & 0.1603 & 0.000 & 28.5 & 25.0 \\
\hline 12.0 & & 27.049 & 34.309 & 22.184 & 0.1660 & 0.000 & 24.3 & 22.3 \\
\hline 13.0 & & 27.044 & 34.335 & 22.205 & 0.1716 & 0.000 & 21.4 & 17.2 \\
\hline 14.0 & & 27.042 & 34.343 & 22.211 & 0.1773 & 0.000 & 19.8 & 15.2 \\
\hline
\end{tabular}
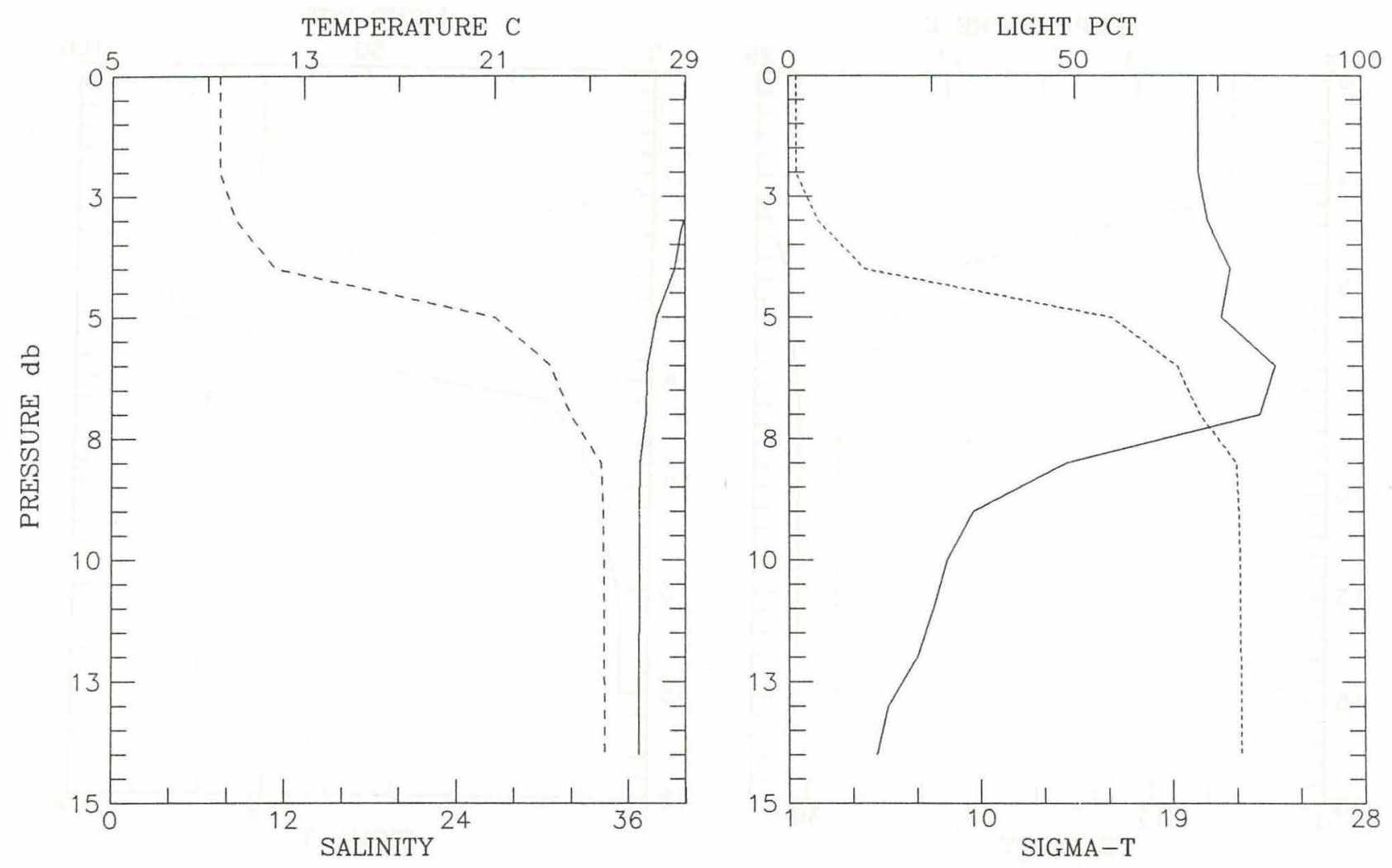


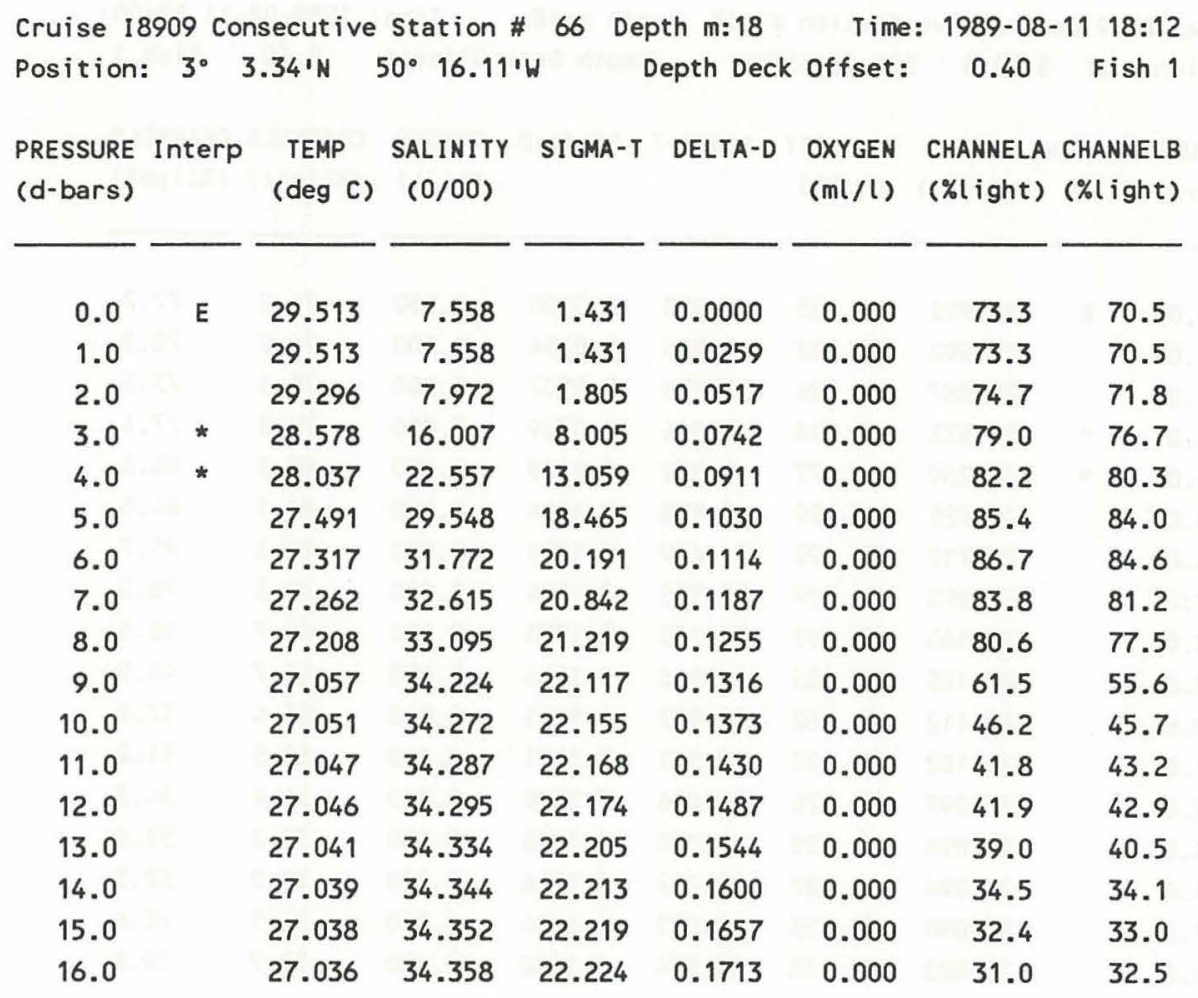
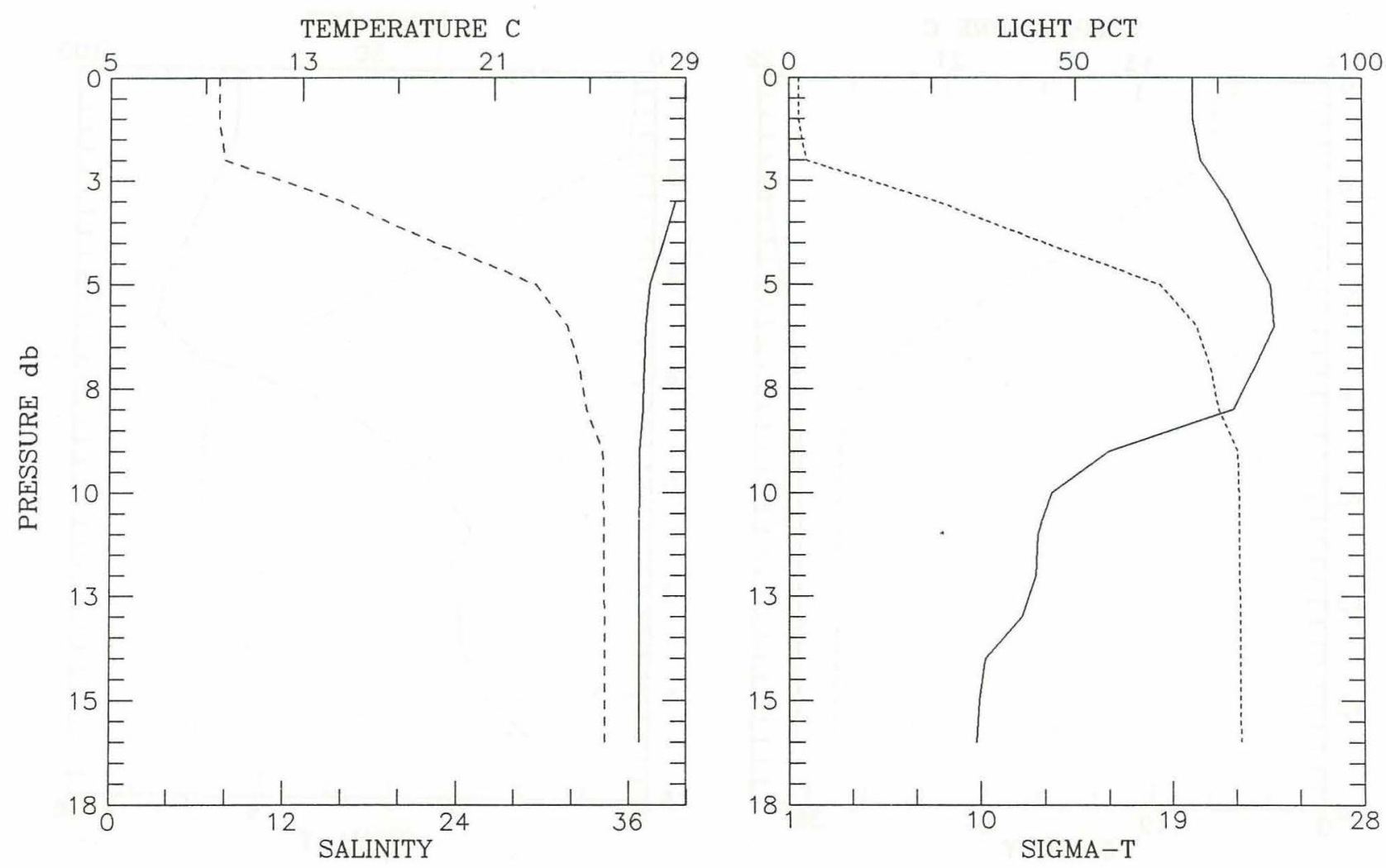


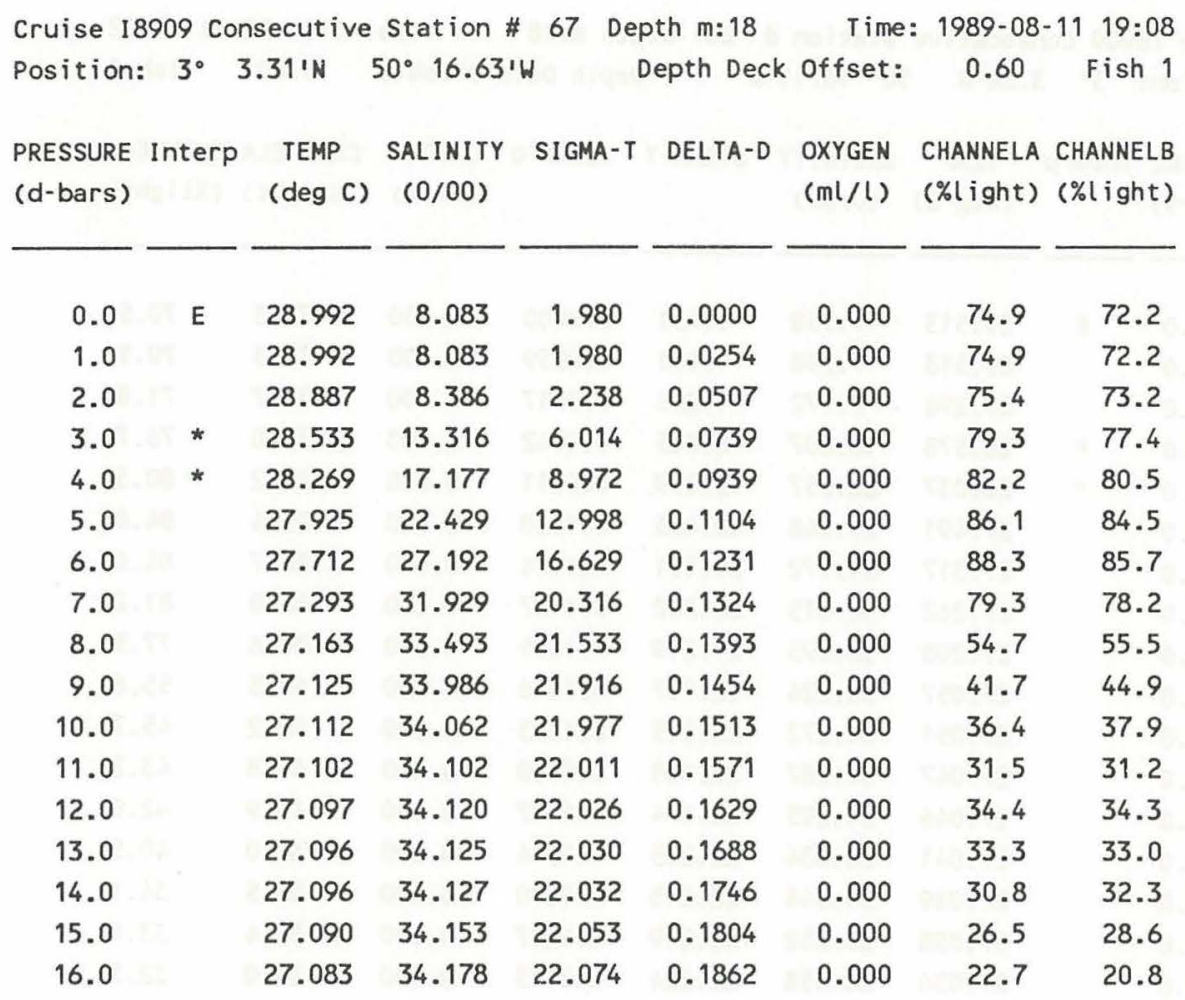
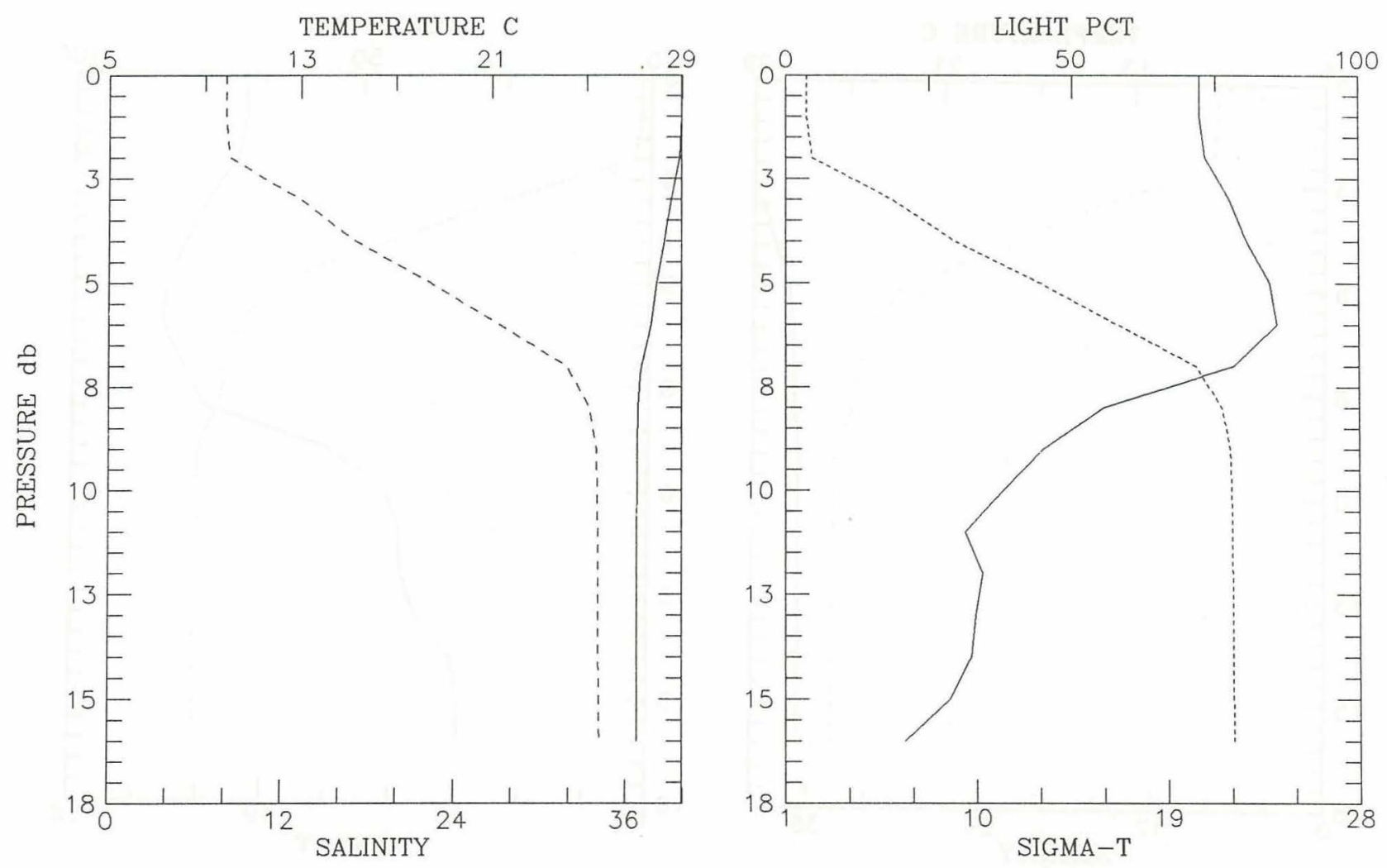


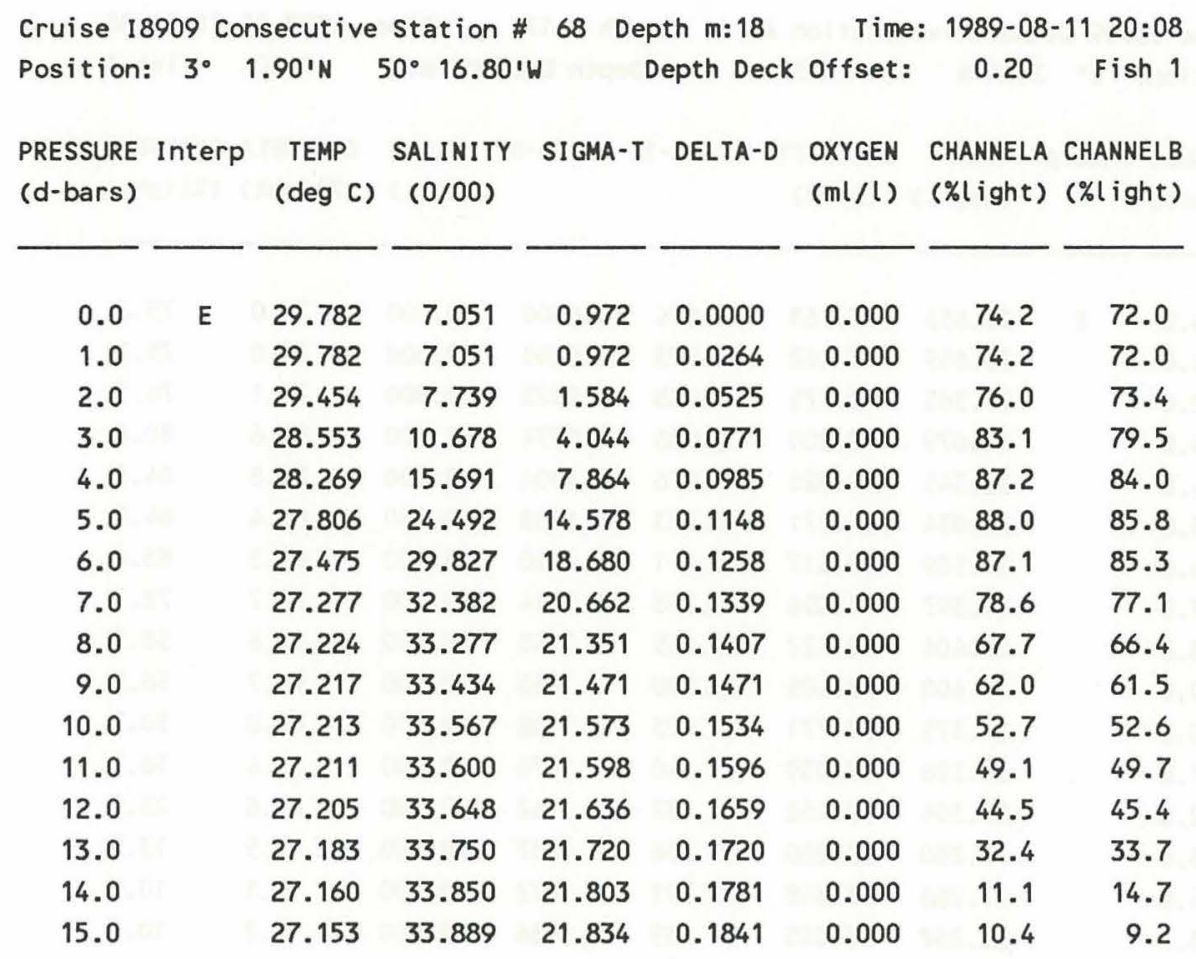
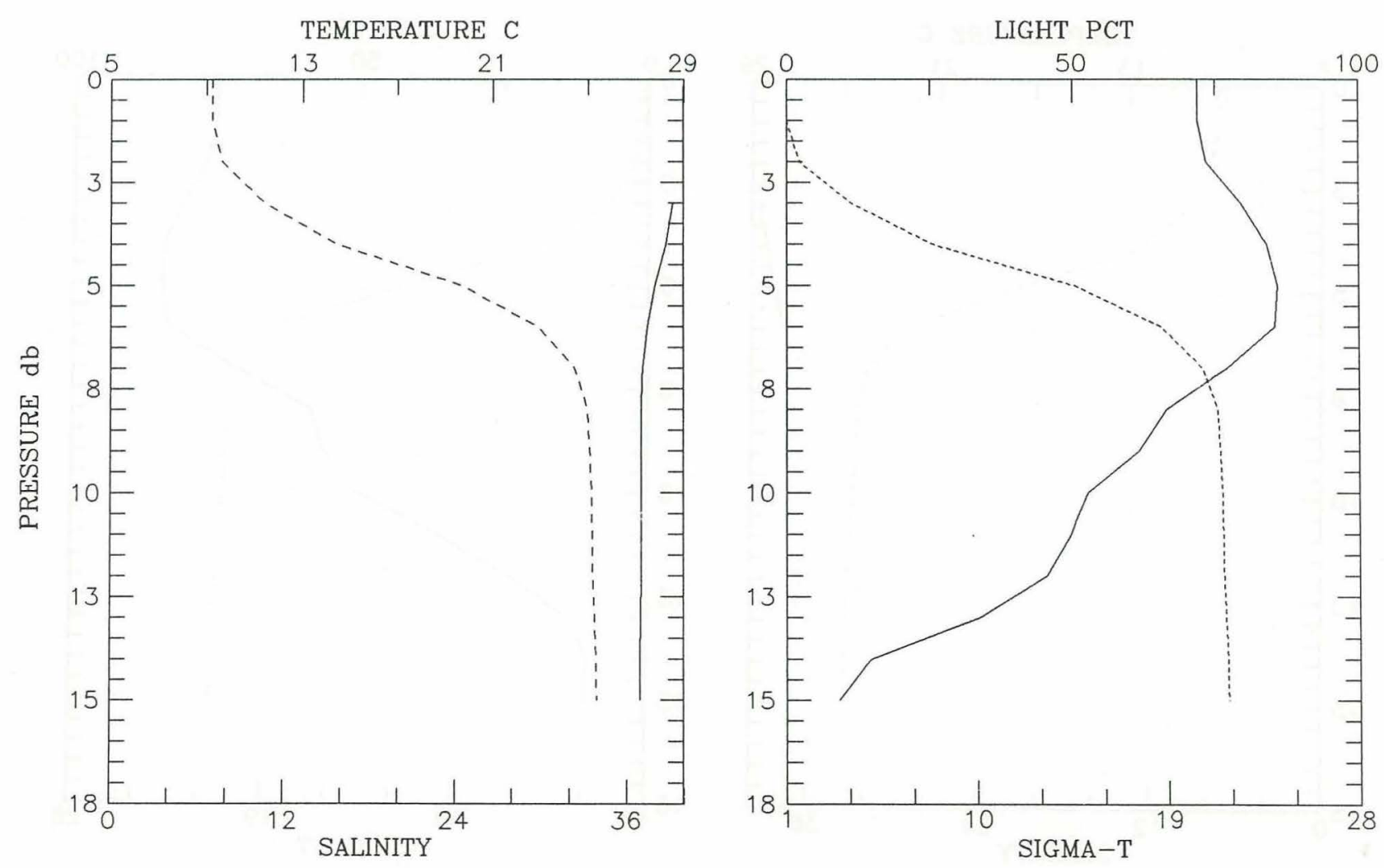


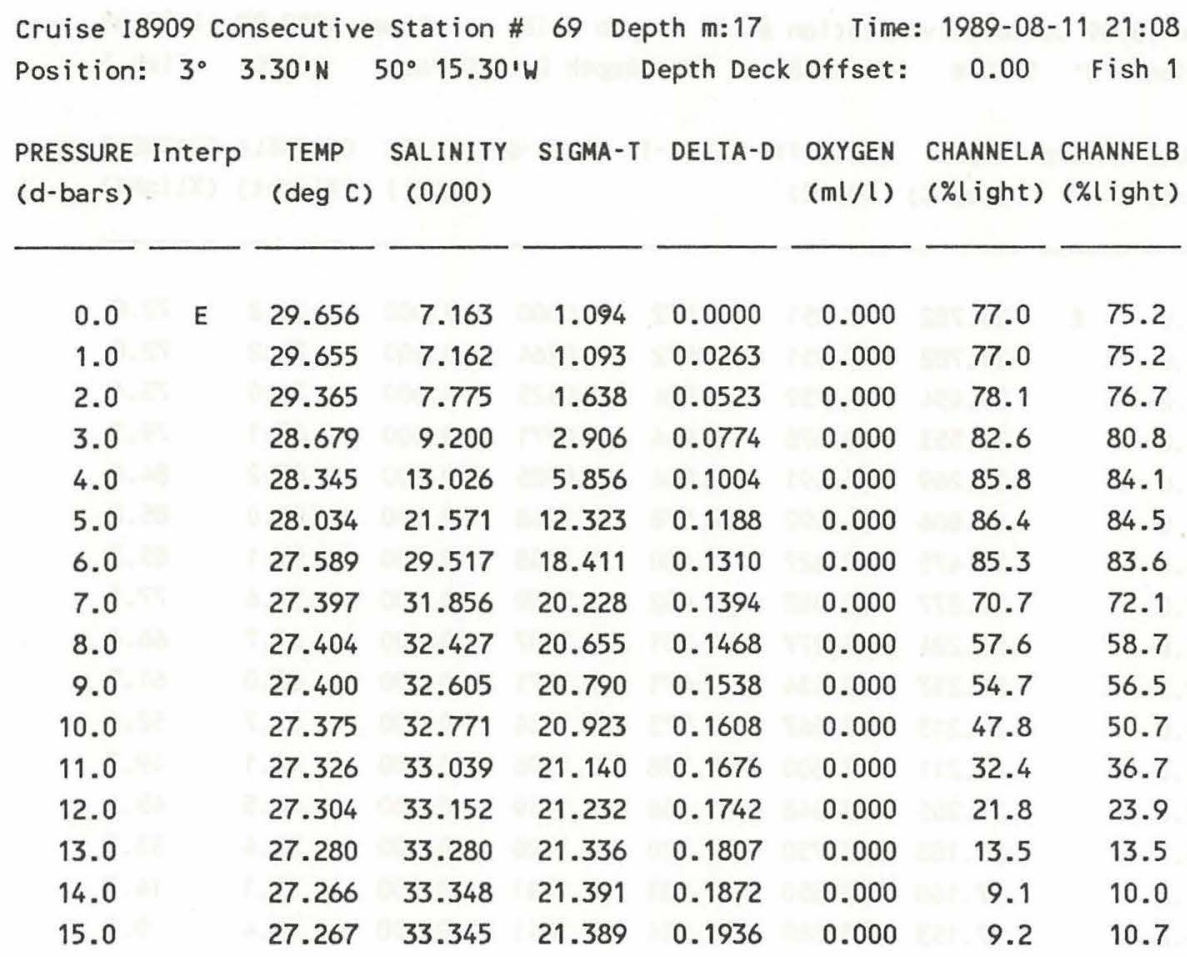
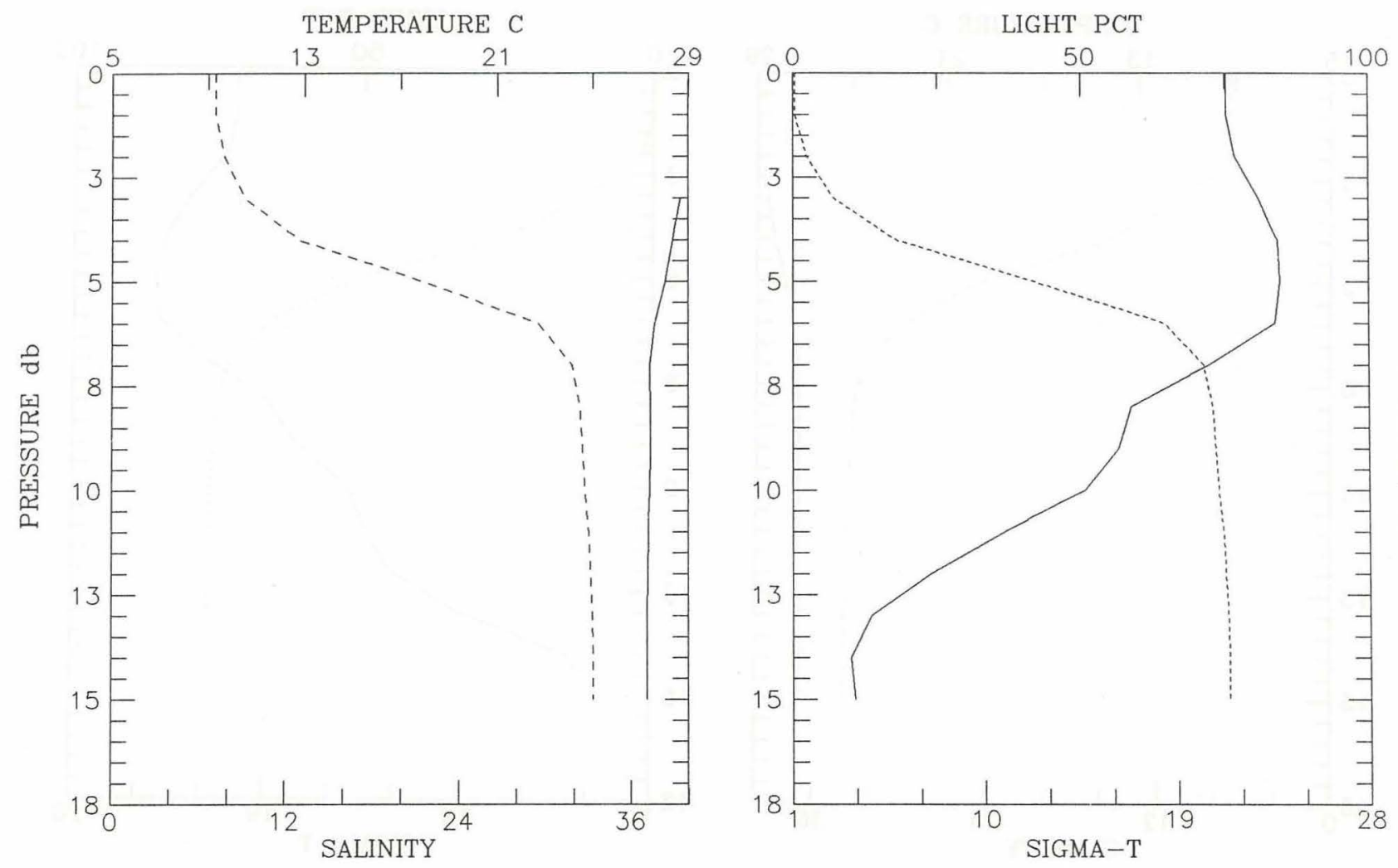


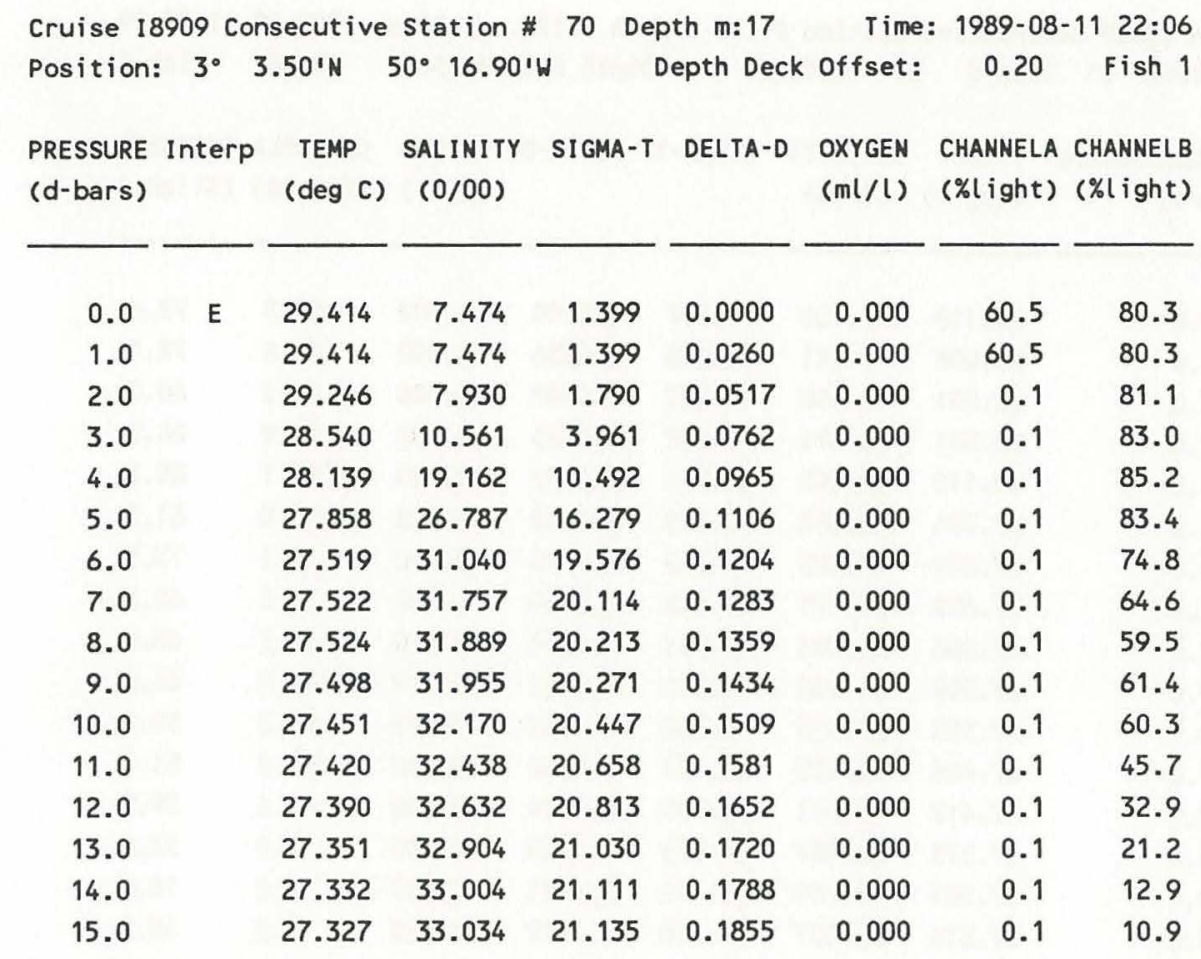
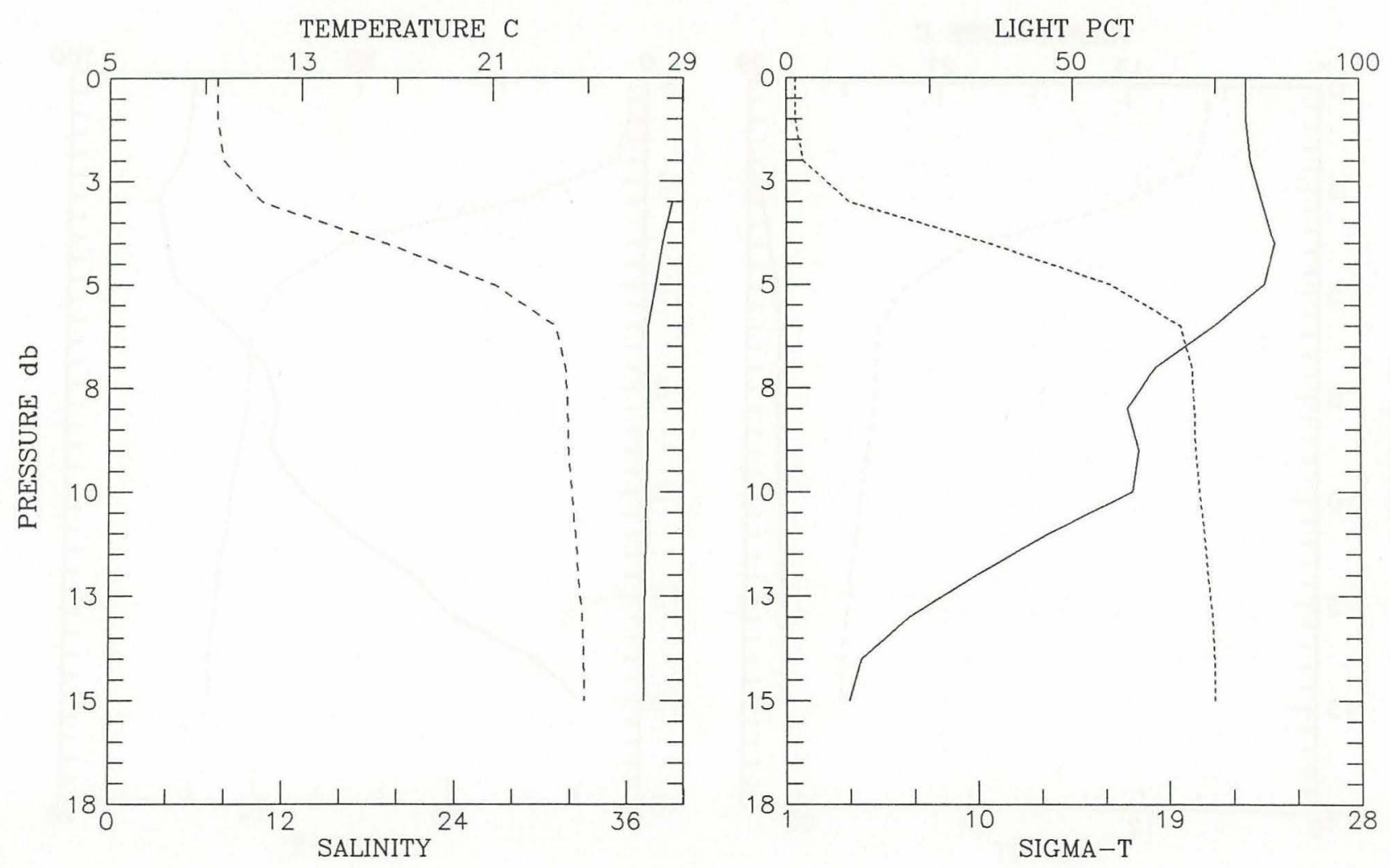


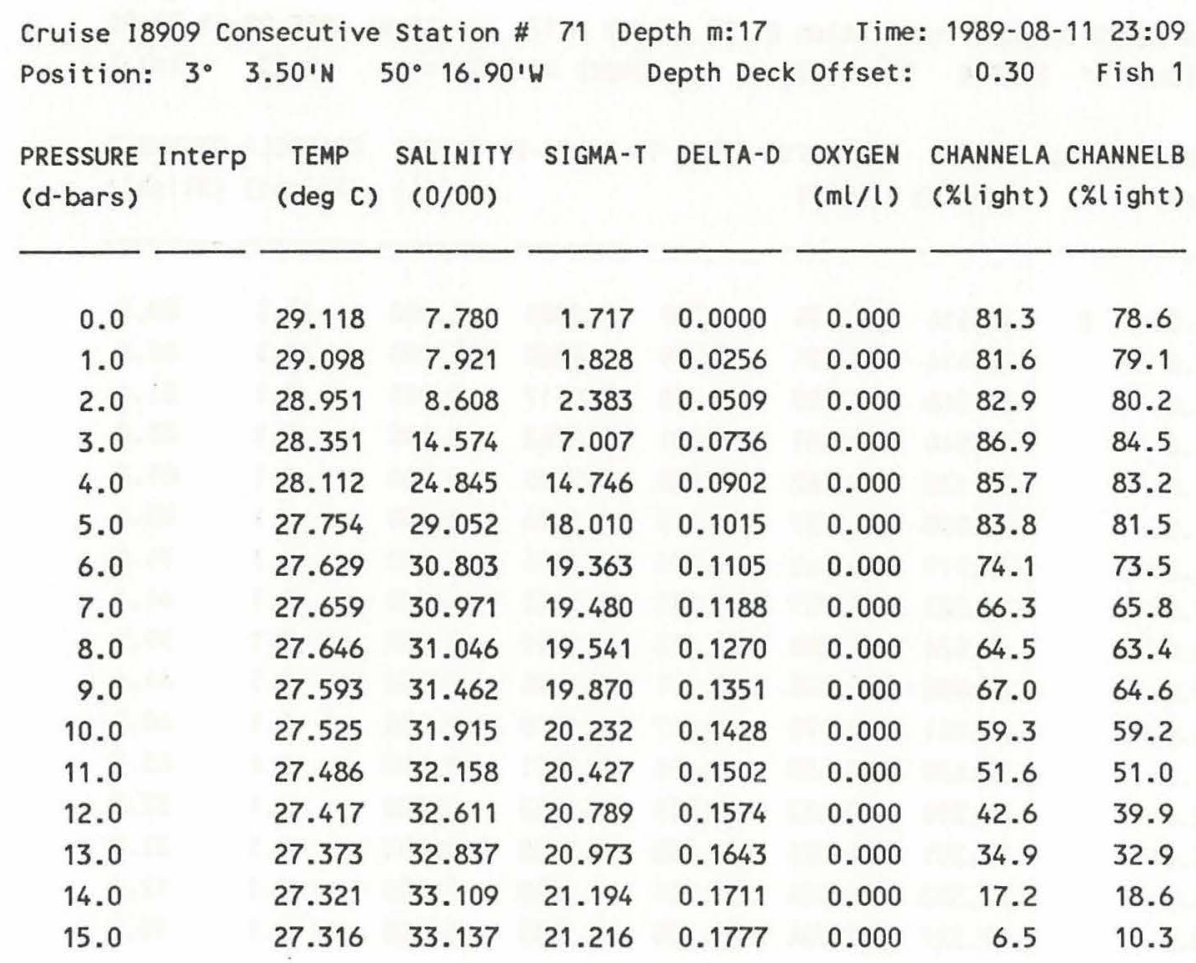
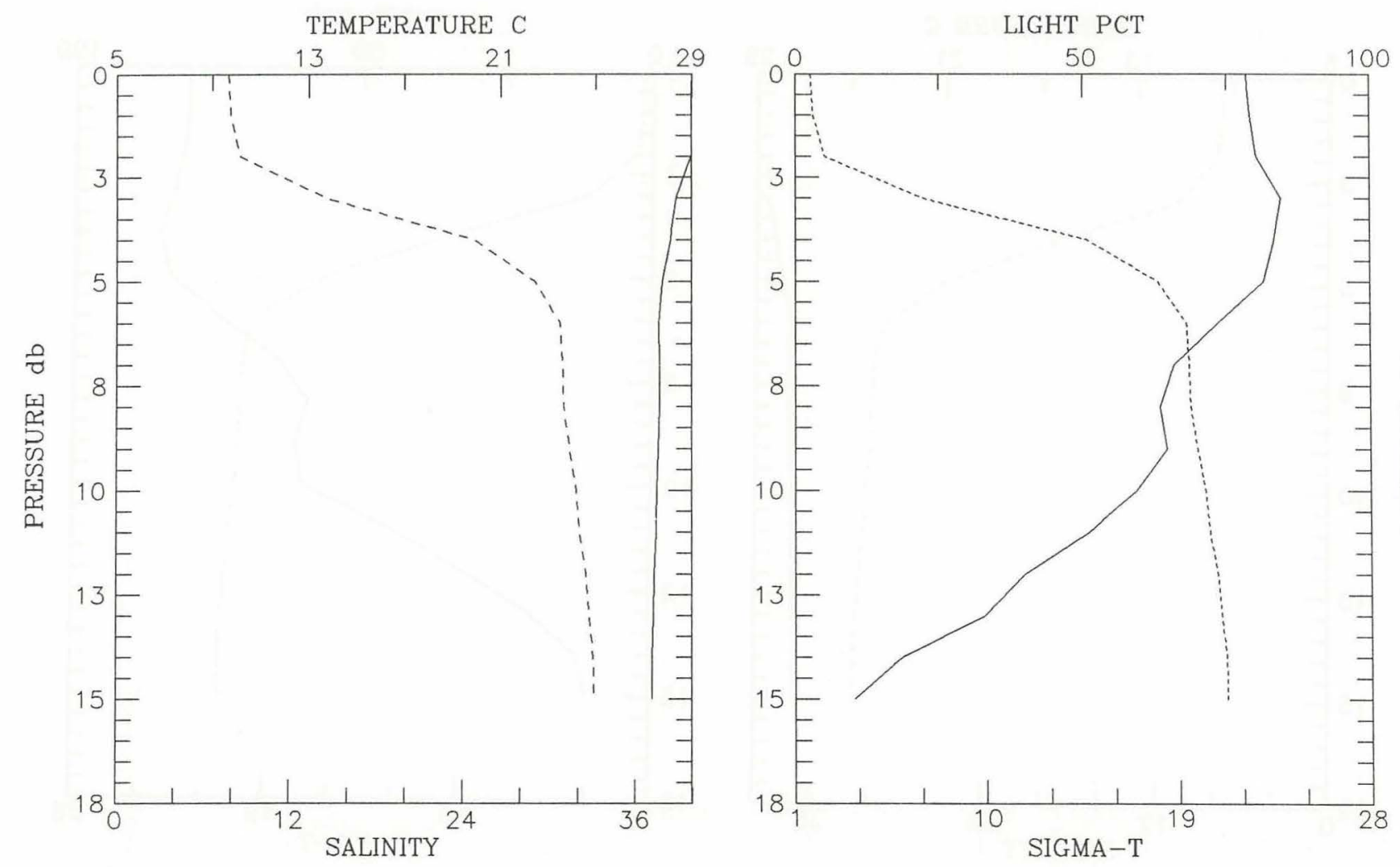


\begin{tabular}{|c|c|c|c|c|c|c|c|c|}
\hline $\begin{array}{l}\text { Cruise } 18 \\
\text { Position: }\end{array}$ & $\begin{array}{l}8909 \mathrm{C} \\
: 3^{\circ}\end{array}$ & $\begin{array}{l}.50^{\prime} \mathrm{N}\end{array}$ & $\begin{array}{l}\text { Station } \\
50^{\circ} 16.90^{\prime}\end{array}$ & 12 & $\begin{array}{l}\text { th } \mathrm{m}: 17 \\
\text { epth Deck }\end{array}$ & $\begin{array}{r}\text { Time: } \\
\text { Offset: }\end{array}$ & $\begin{array}{c}1989-08 \\
0.20\end{array}$ & Fish 1 \\
\hline $\begin{array}{l}\text { PRESSURE } \\
\text { (d-bars) }\end{array}$ & Interp & $\begin{array}{c}\text { TEMP } \\
(\operatorname{deg} C)\end{array}$ & $\begin{array}{c}\text { SALINITY } \\
(0 / 00)\end{array}$ & SIGMA-T & DELTA-D & $\begin{array}{l}\text { OXYGEN } \\
(\mathrm{ml} / \mathrm{l})\end{array}$ & $\begin{array}{l}\text { CHANNELA } \\
\text { (\%light) }\end{array}$ & $\begin{array}{l}\text { CHANNELB } \\
\text { (\%light) }\end{array}$ \\
\hline 0.0 & E & 29.046 & 7.374 & 1.437 & 0.0000 & 0.000 & 81.2 & 78.6 \\
\hline 1.0 & & 29.046 & 7.374 & 1.437 & 0.0259 & 0.000 & 81.2 & 78.6 \\
\hline 2.0 & & 28.948 & 8.376 & 2.212 & 0.0515 & 0.000 & 82.6 & 79.8 \\
\hline 3.0 & & 28.640 & 11.103 & 4.334 & 0.0756 & 0.000 & 84.8 & 82.5 \\
\hline 4.0 & & 28.149 & 25.921 & 15.539 & 0.0931 & 0.000 & 83.0 & 83.5 \\
\hline 5.0 & & 27.808 & 29.454 & 18.294 & 0.1039 & 0.000 & 82.3 & 80.1 \\
\hline 6.0 & & 27.810 & 29.771 & 18.531 & 0.1132 & 0.000 & 75.1 & 72.8 \\
\hline 7.0 & & 27.809 & 29.959 & 18.673 & 0.1223 & 0.000 & 69.6 & 66.8 \\
\hline 8.0 & & 27.759 & 30.374 & 19.000 & 0.1312 & 0.000 & 68.2 & 66.3 \\
\hline 9.0 & & 27.675 & 30.743 & 19.304 & 0.1397 & 0.000 & 69.5 & 65.8 \\
\hline 10.0 & & 27.617 & 31.275 & 19.722 & 0.1480 & 0.000 & 69.0 & 66.8 \\
\hline 11.0 & & 27.499 & 32.149 & 20.415 & 0.1557 & 0.000 & 60.8 & 60.9 \\
\hline 12.0 & & 27.406 & 32.688 & 20.850 & 0.1628 & 0.000 & 52.0 & 52.3 \\
\hline 13.0 & & 27.353 & 33.001 & 21.102 & 0.1697 & 0.000 & 47.8 & 46.2 \\
\hline 14.0 & & 27.298 & 33.259 & 21.314 & 0.1763 & 0.000 & 24.5 & 29.4 \\
\hline 15.0 & & 27.265 & 33.399 & 21.430 & 0.1827 & 0.000 & 5.2 & 5.4 \\
\hline
\end{tabular}
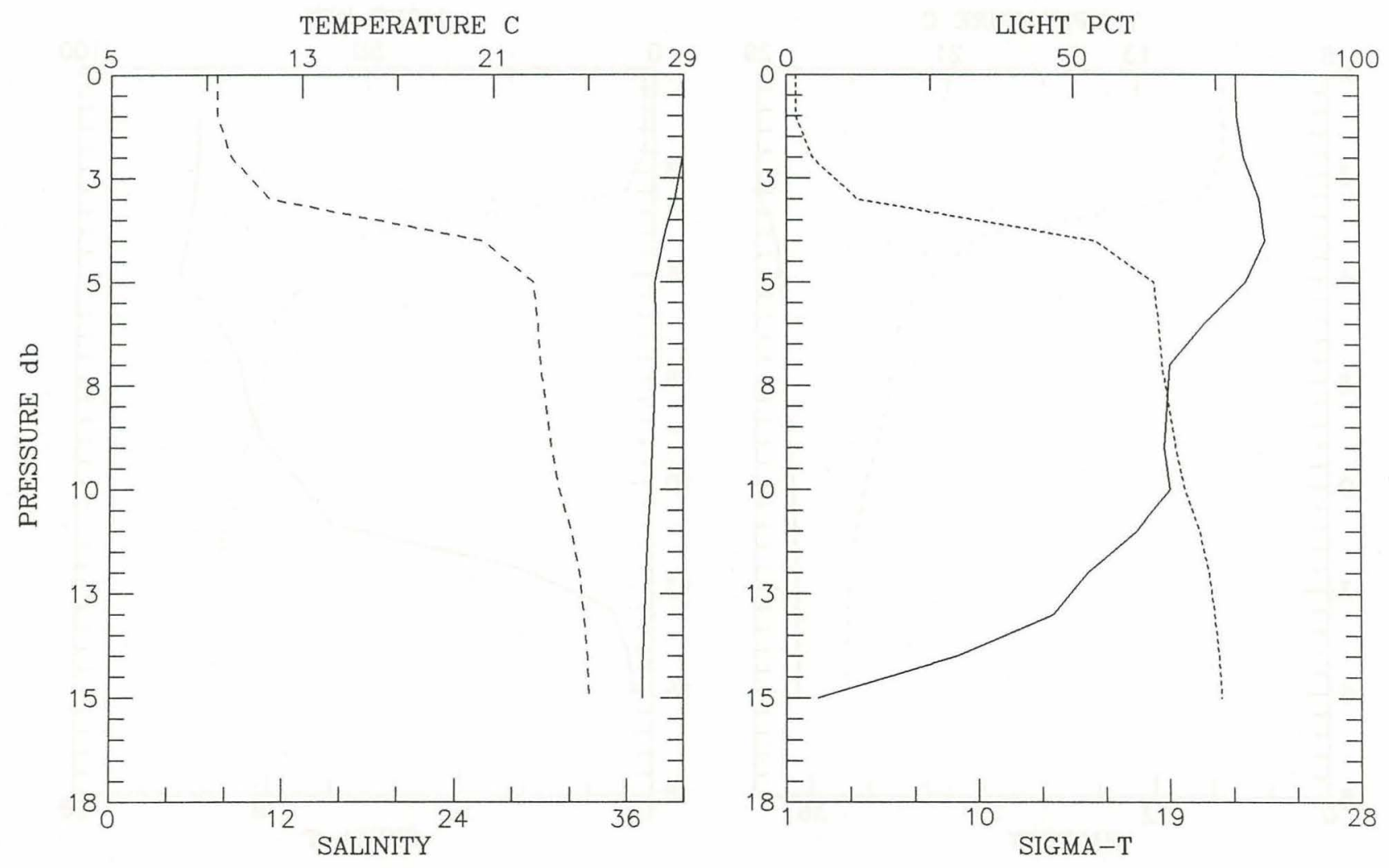


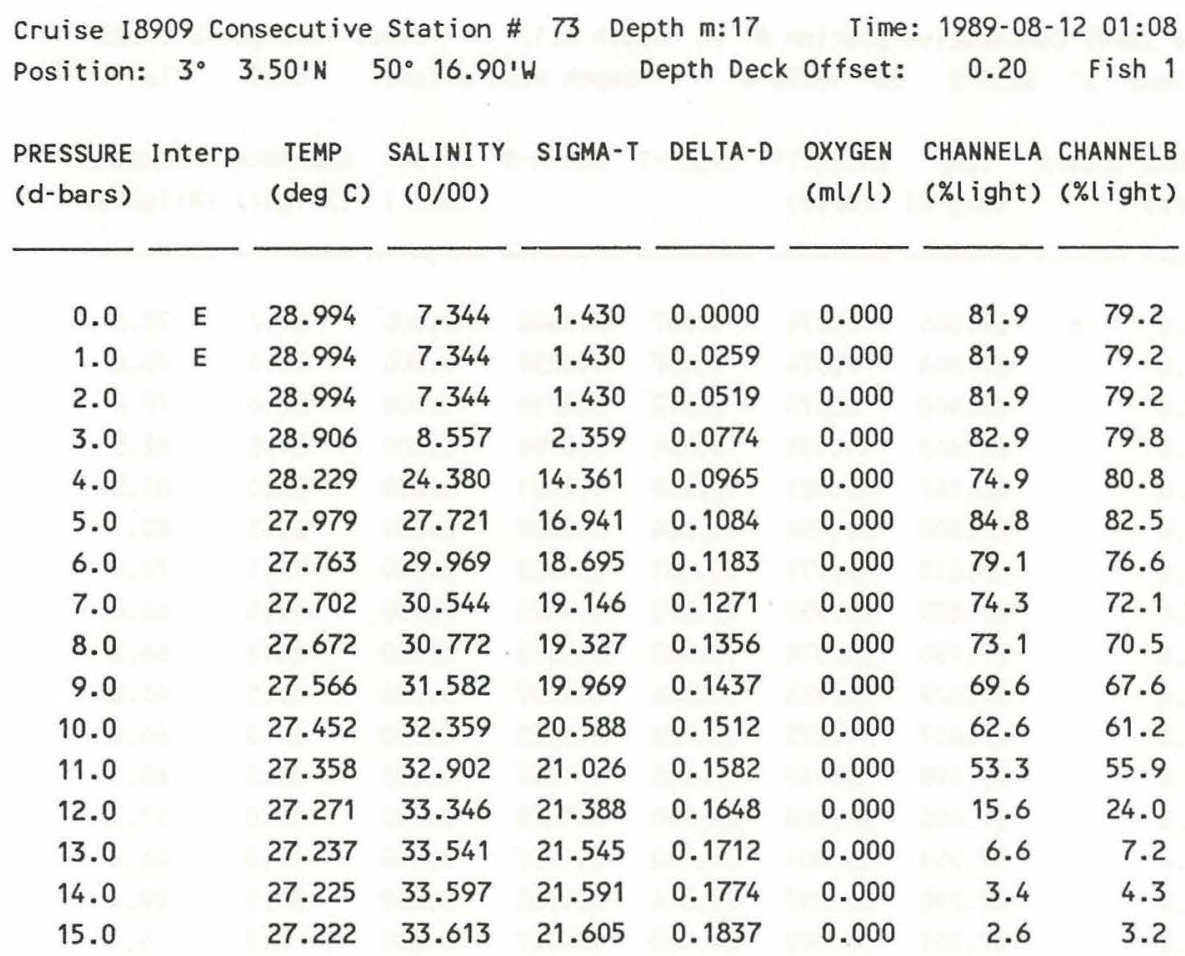
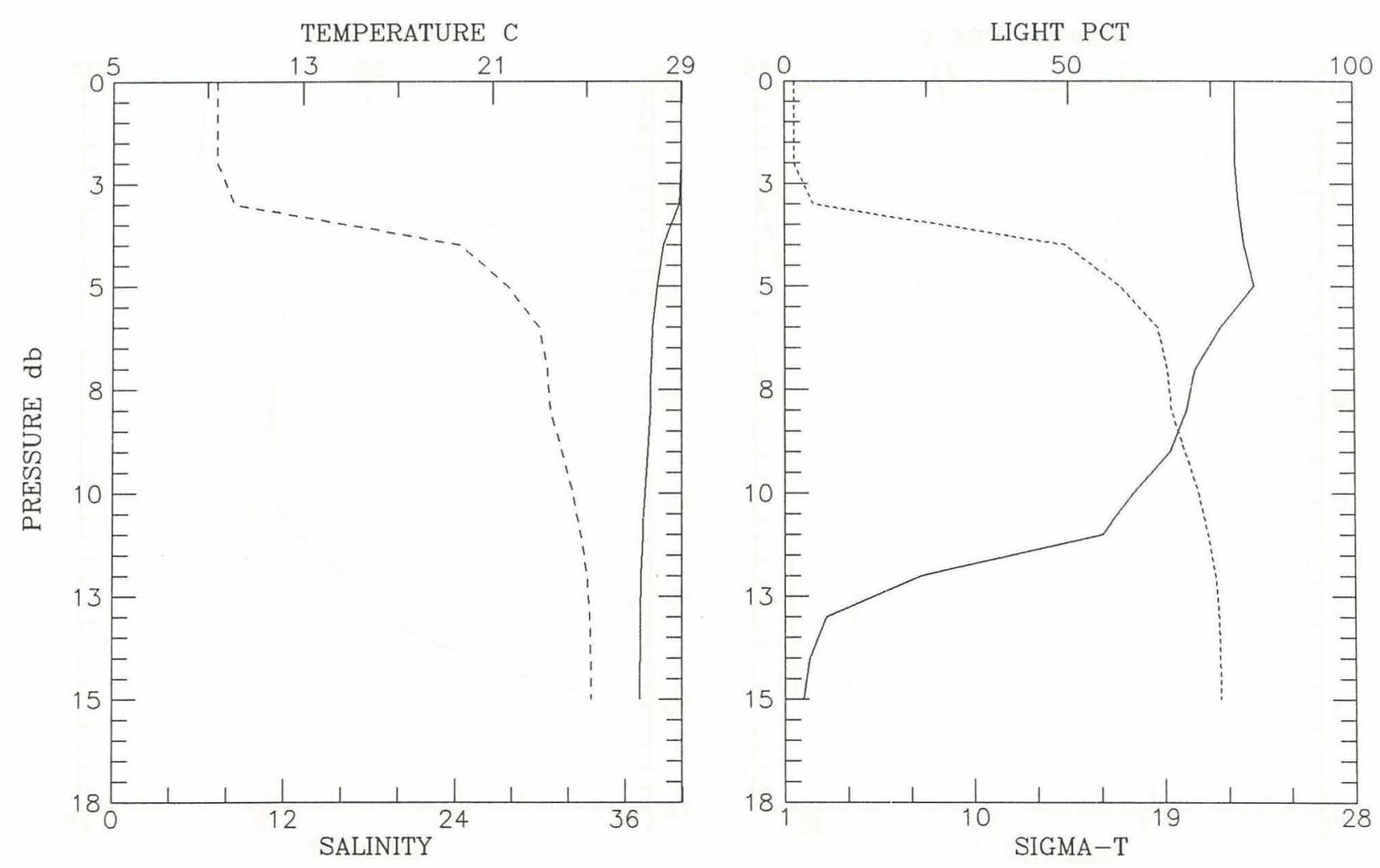


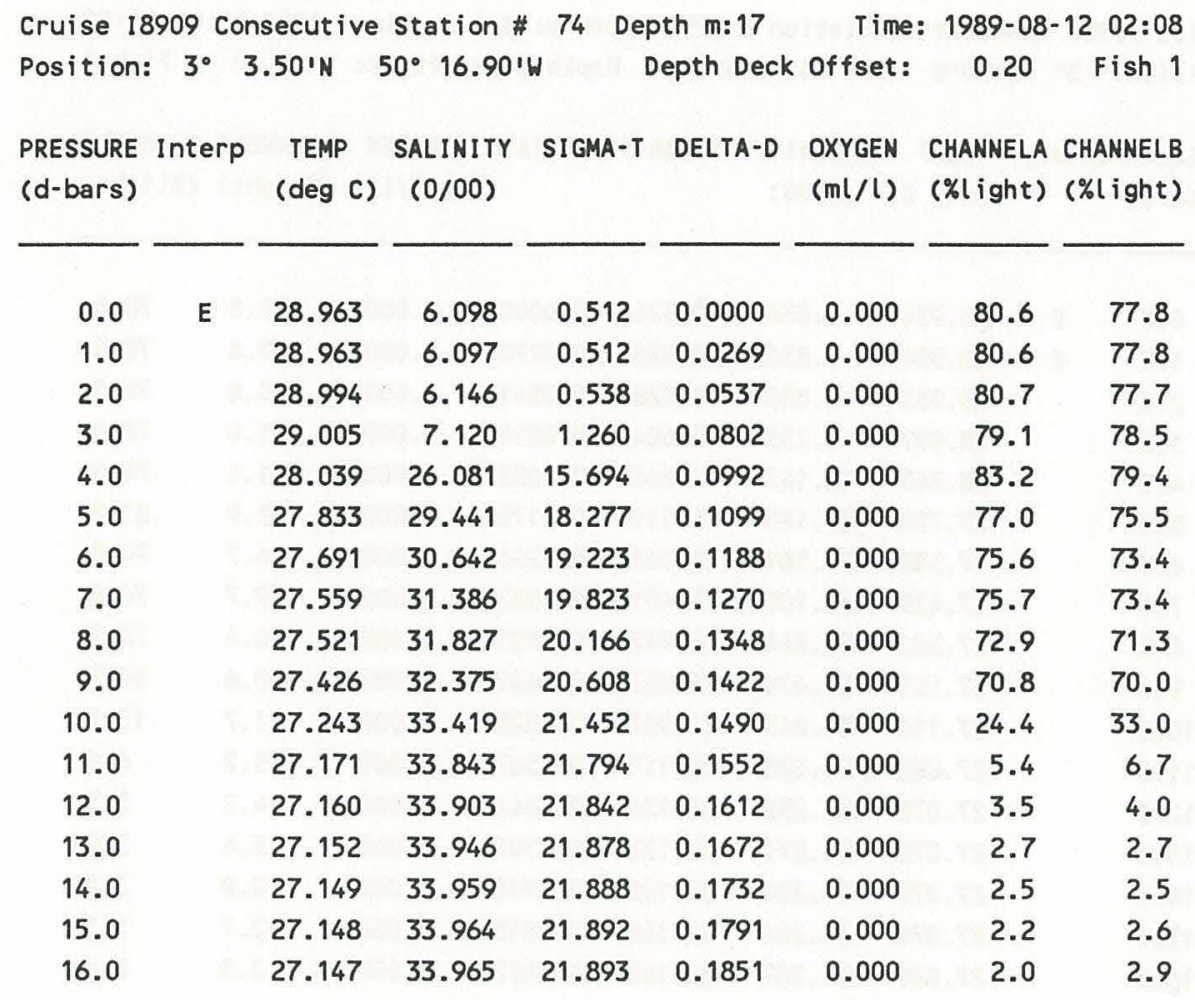
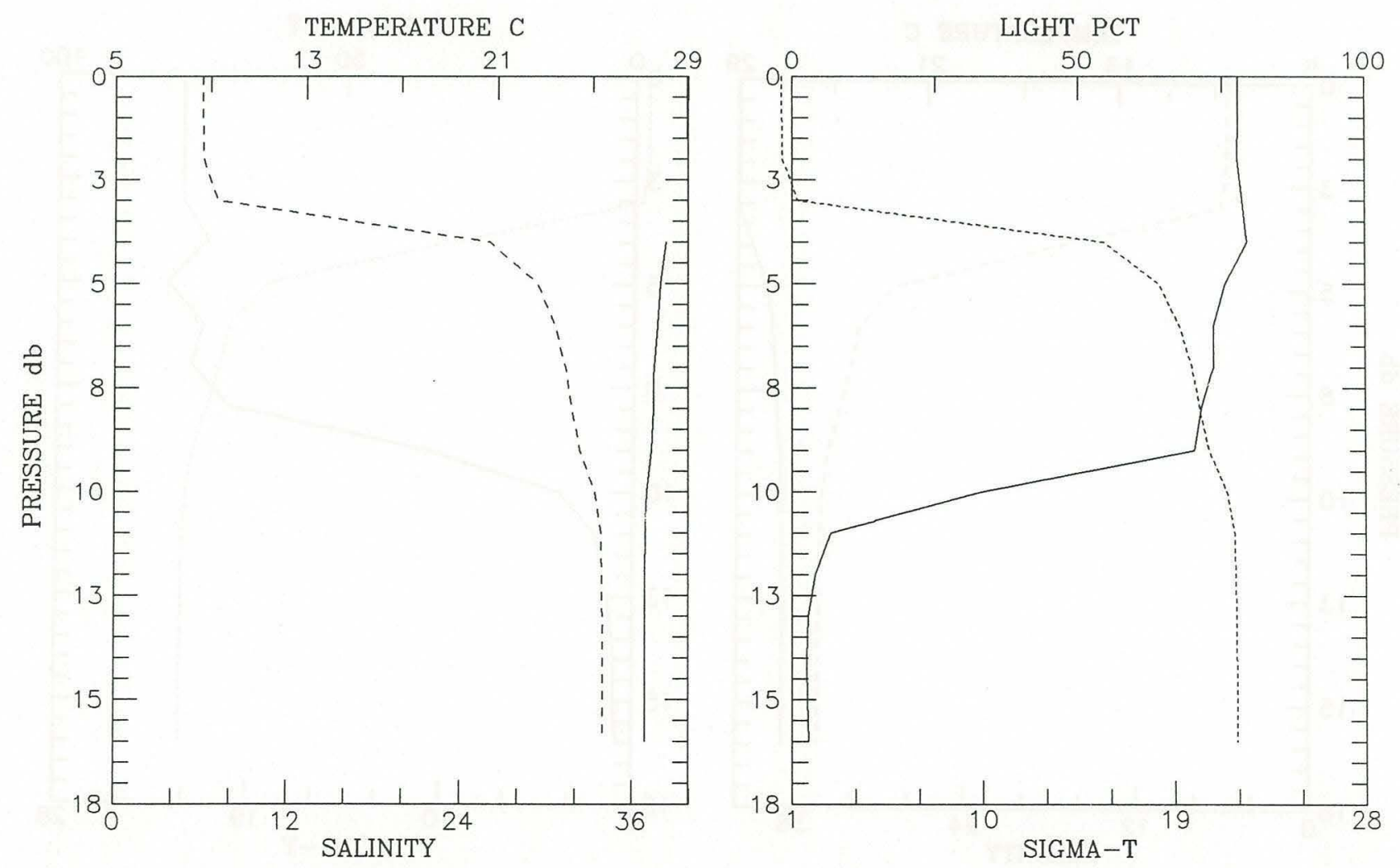


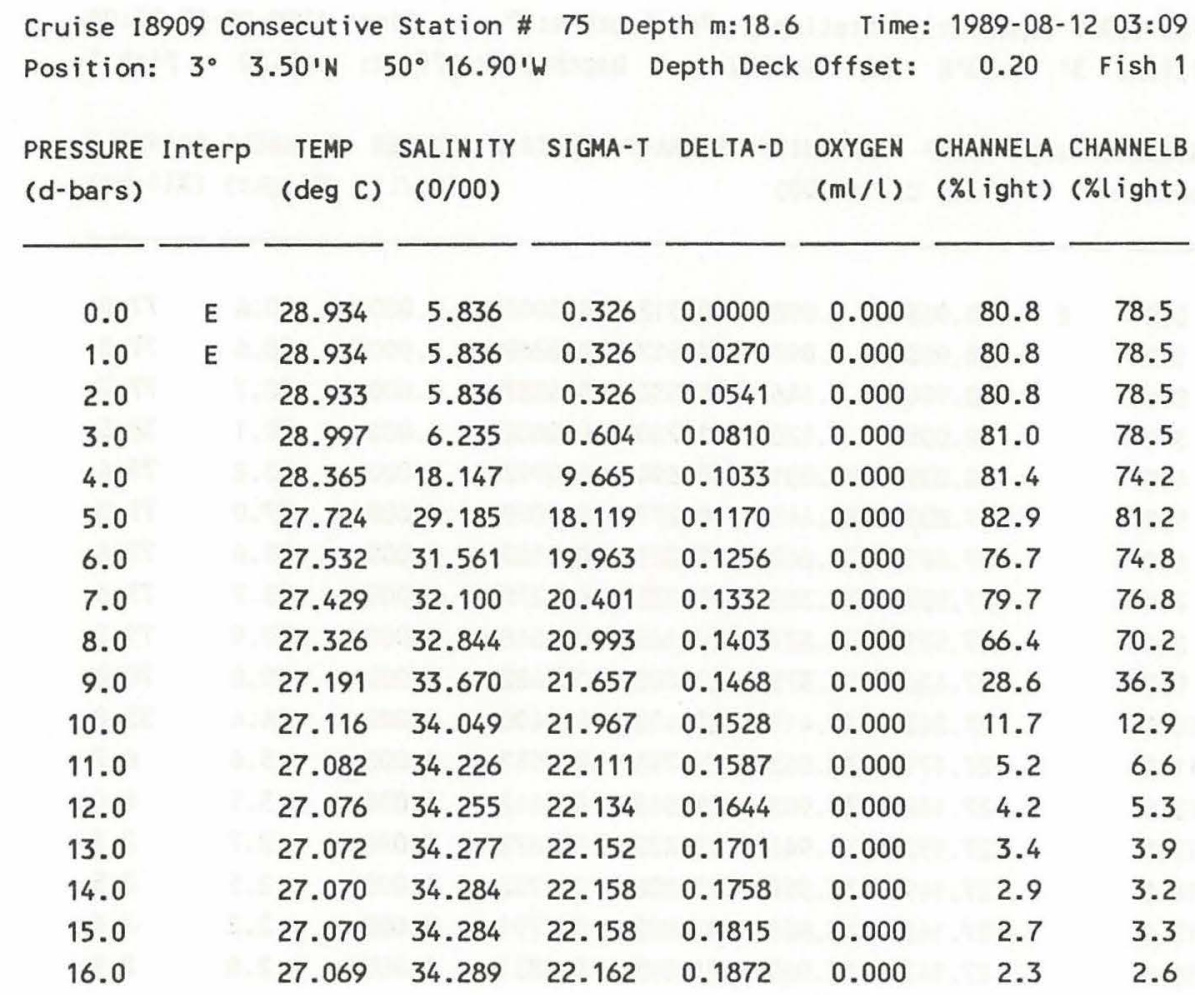
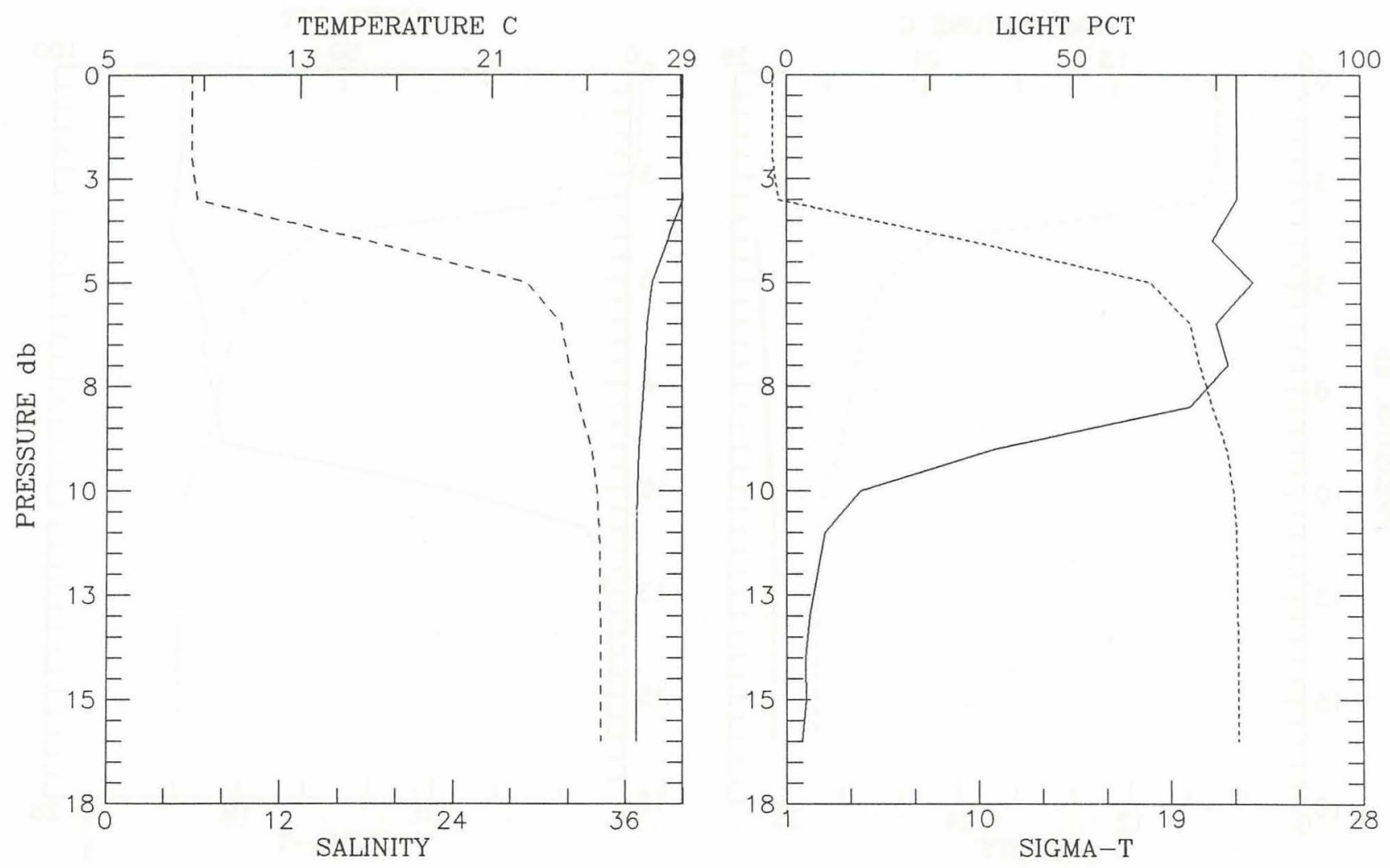


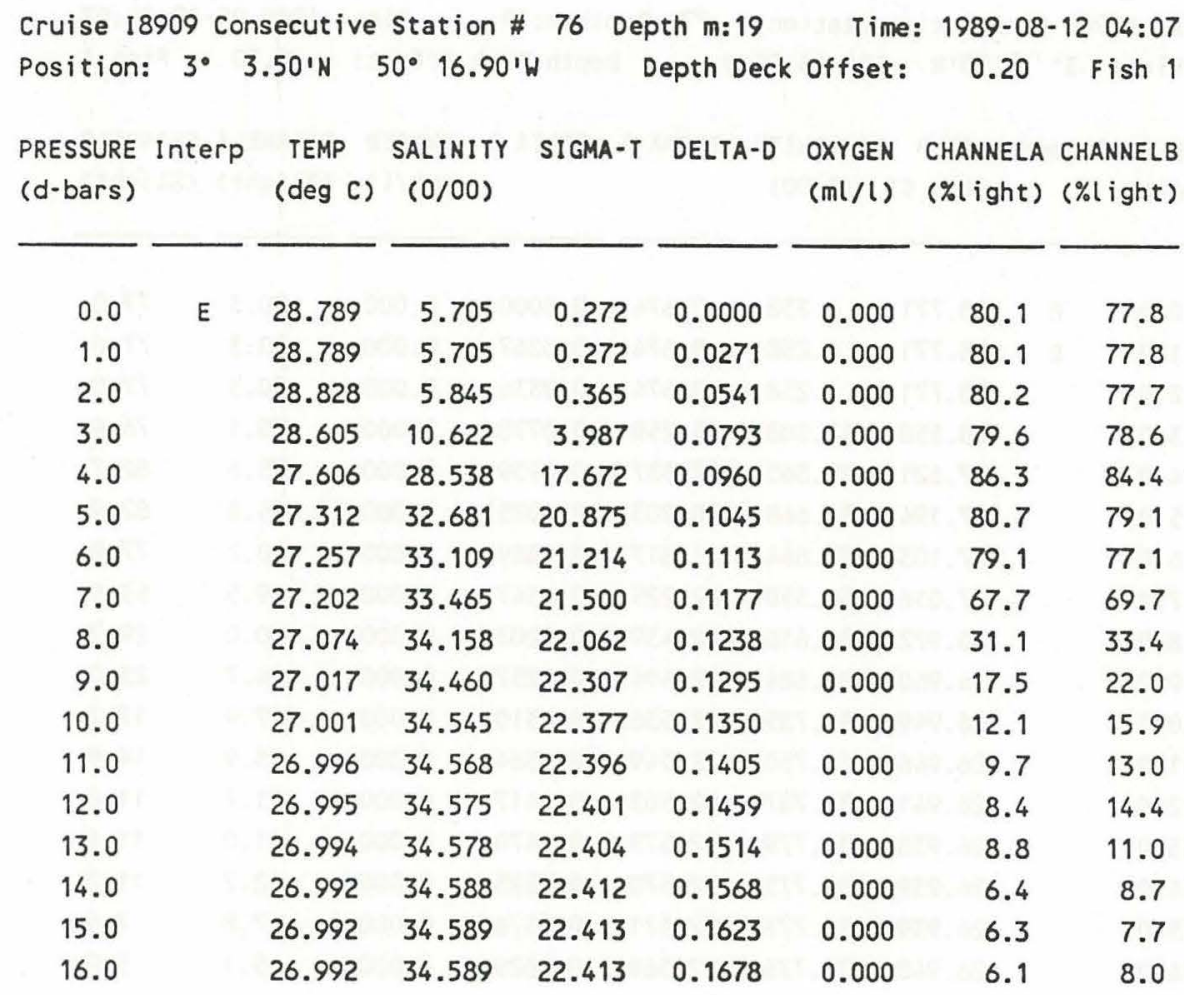
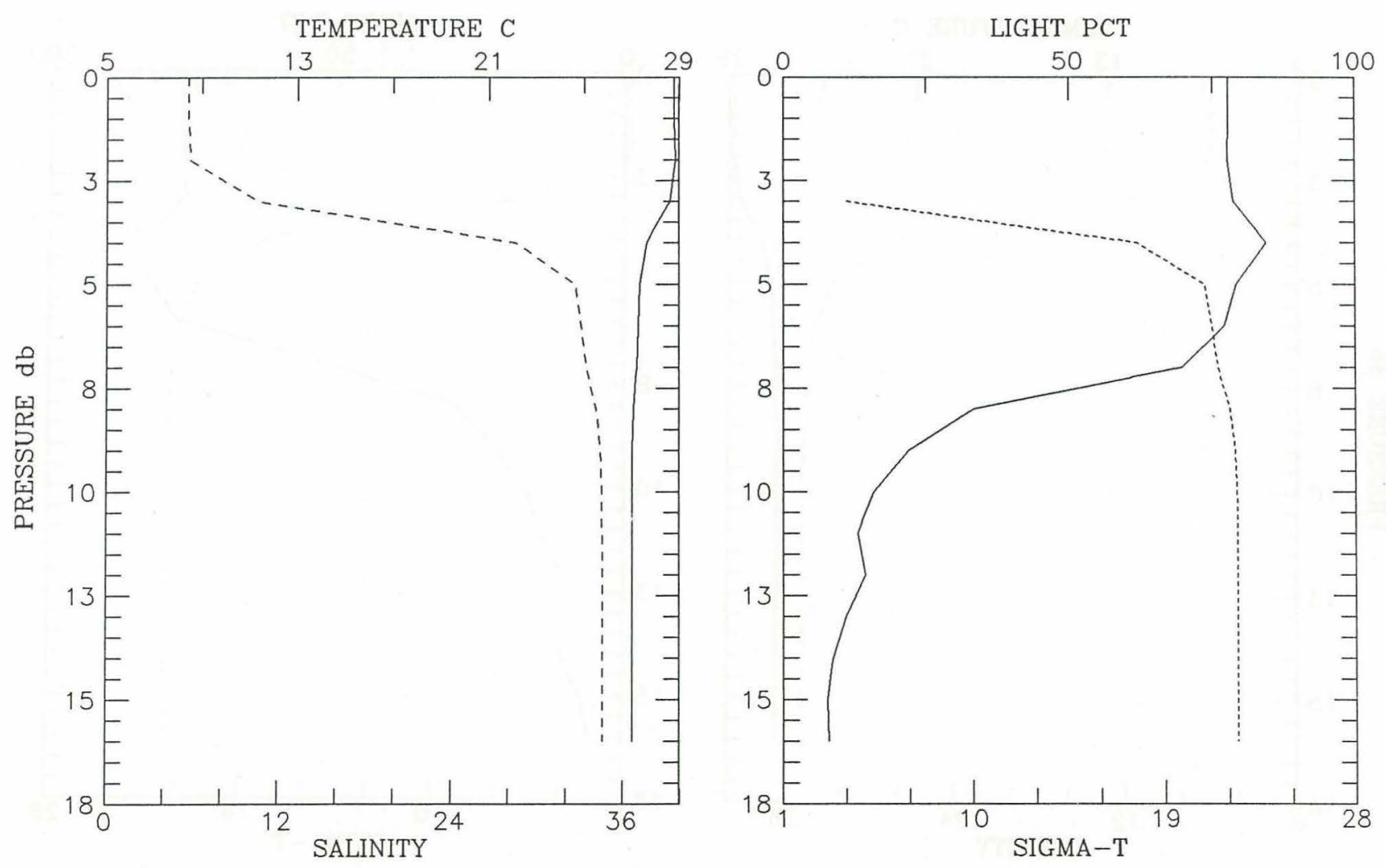
Cruise 18909 Consecutive Station \# 77 Depth m:19 Time: 1989-08-12 05:07 Position: $3^{\circ} 3.50^{\prime} \mathrm{N} 50^{\circ} 16.90 \mathrm{~W} \quad$ Depth Deck offset: $0.20 \quad$ Fish 1

\begin{tabular}{|c|c|c|c|c|c|c|c|c|}
\hline $\begin{array}{l}\text { PRESSURE } \\
\text { (d-bars) }\end{array}$ & Interp & $\begin{array}{c}\text { TEMP } \\
(\operatorname{deg} \mathrm{C})\end{array}$ & $\begin{array}{l}\text { SALINITY } \\
(0 / 00)\end{array}$ & SIGMA-T & DELTA-D & $\begin{array}{l}\text { OXYGEN } \\
(\mathrm{ml} / \mathrm{l})\end{array}$ & $\begin{array}{l}\text { CHANNELA } \\
\text { (\%light) }\end{array}$ & $\begin{array}{l}\text { CHANNELB } \\
\text { (\%light) }\end{array}$ \\
\hline 0.0 & $E$ & 28.771 & 6.238 & 0.674 & 0.0000 & 0.000 & 80.3 & 77.0 \\
\hline 1.0 & E & 28.771 & 6.238 & 0.674 & 0.0267 & 0.000 & 80.3 & 77.0 \\
\hline 2.0 & & 28.771 & 6.238 & 0.674 & 0.0534 & 0.000 & 80.3 & 77.0 \\
\hline 3.0 & & 28.550 & 12.308 & 5.259 & 0.0778 & 0.000 & 70.1 & 76.4 \\
\hline 4.0 & & 27.621 & 28.365 & 17.537 & 0.0939 & 0.000 & 75.6 & 82.7 \\
\hline 5.0 & & 27.194 & 32.668 & 20.903 & 0.1025 & 0.000 & 85.8 & 82.9 \\
\hline 6.0 & & 27.103 & 33.844 & 21.817 & 0.1089 & 0.000 & 80.2 & 77.9 \\
\hline 7.0 & & 27.016 & 34.350 & 22.225 & 0.1147 & 0.000 & 49.5 & 53.0 \\
\hline 8.0 & & 26.972 & 34.616 & 22.439 & 0.1203 & 0.000 & 30.0 & 29.7 \\
\hline 9.0 & & 26.960 & 34.684 & 22.494 & 0.1257 & 0.000 & 24.7 & 23.0 \\
\hline 10.0 & & 26.949 & 34.735 & 22.536 & 0.1310 & 0.000 & 17.9 & 17.2 \\
\hline 11.0 & & 26.946 & 34.750 & 22.549 & 0.1364 & 0.000 & 13.9 & 14.9 \\
\hline 12.0 & & 26.941 & 34.767 & 22.563 & 0.1417 & 0.000 & 11.7 & 11.0 \\
\hline 13.0 & & 26.938 & 34.779 & 22.573 & 0.1470 & 0.000 & 11.0 & 11.4 \\
\hline 14.0 & & 26.939 & 34.775 & 22.570 & 0.1523 & 0.000 & 12.2 & 11.2 \\
\hline 15.0 & & 26.939 & 34.777 & 22.571 & 0.1576 & 0.000 & 7.8 & 7.5 \\
\hline 16.0 & & 26.940 & 34.775 & 22.569 & 0.1629 & 0.000 & 5.1 & 5.9 \\
\hline
\end{tabular}
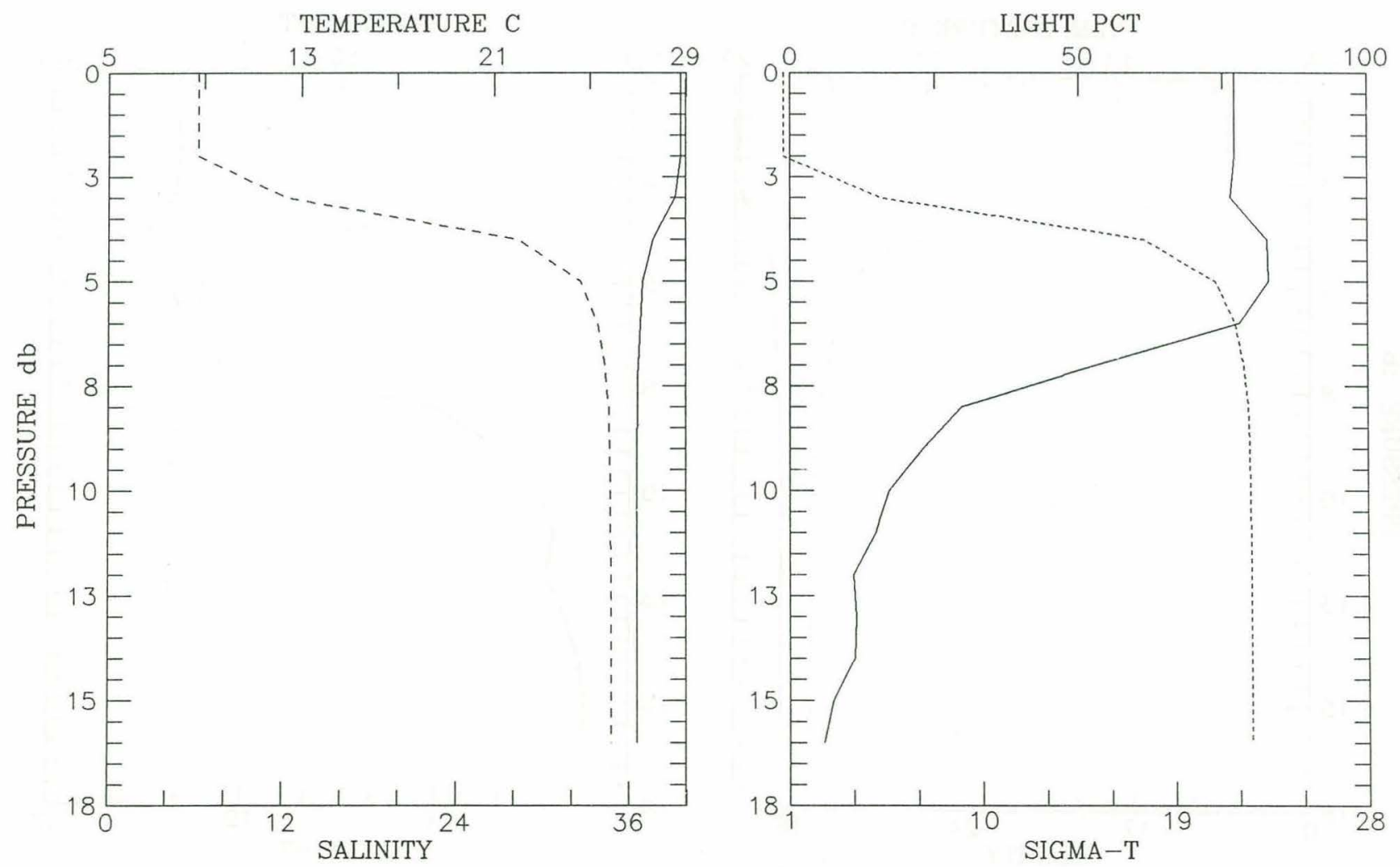


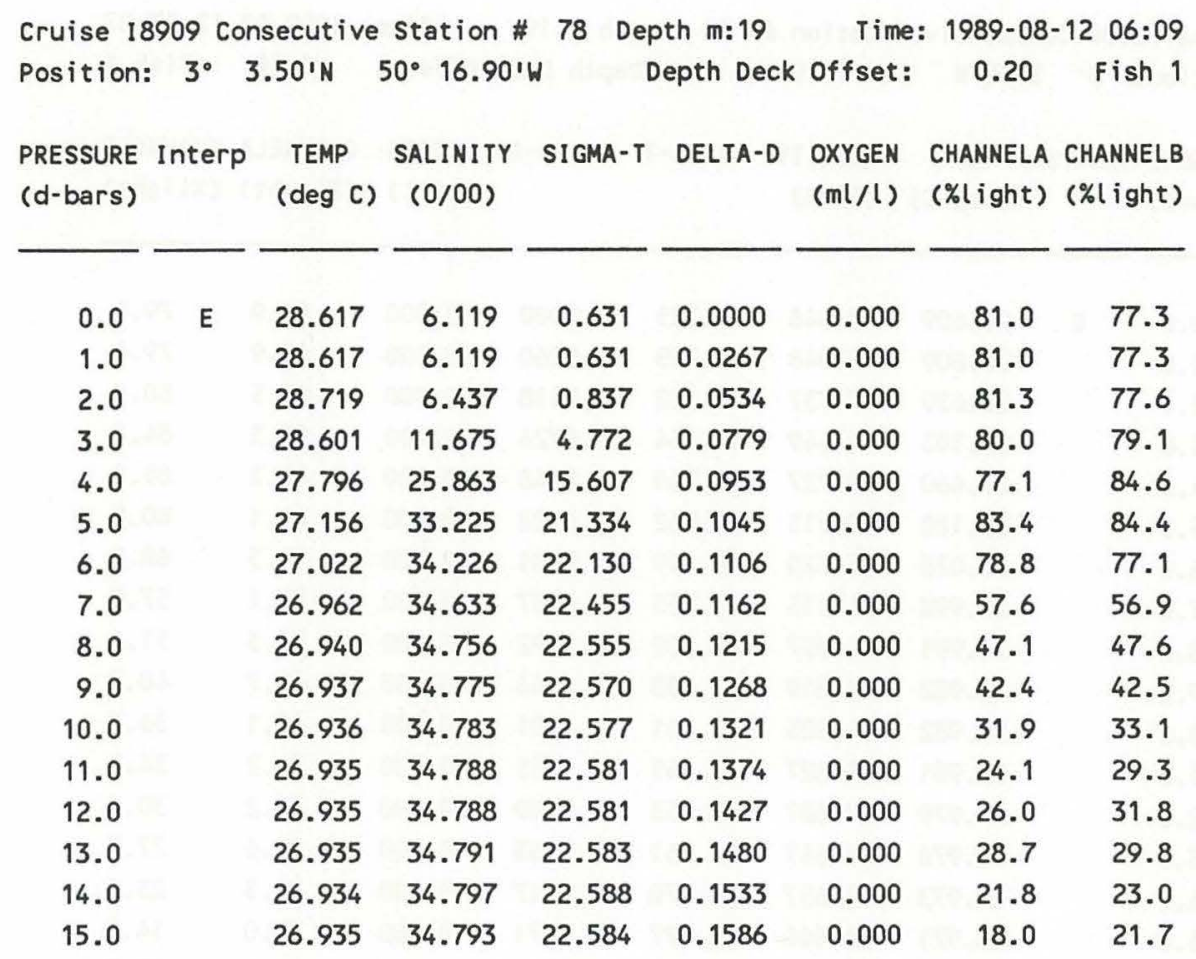
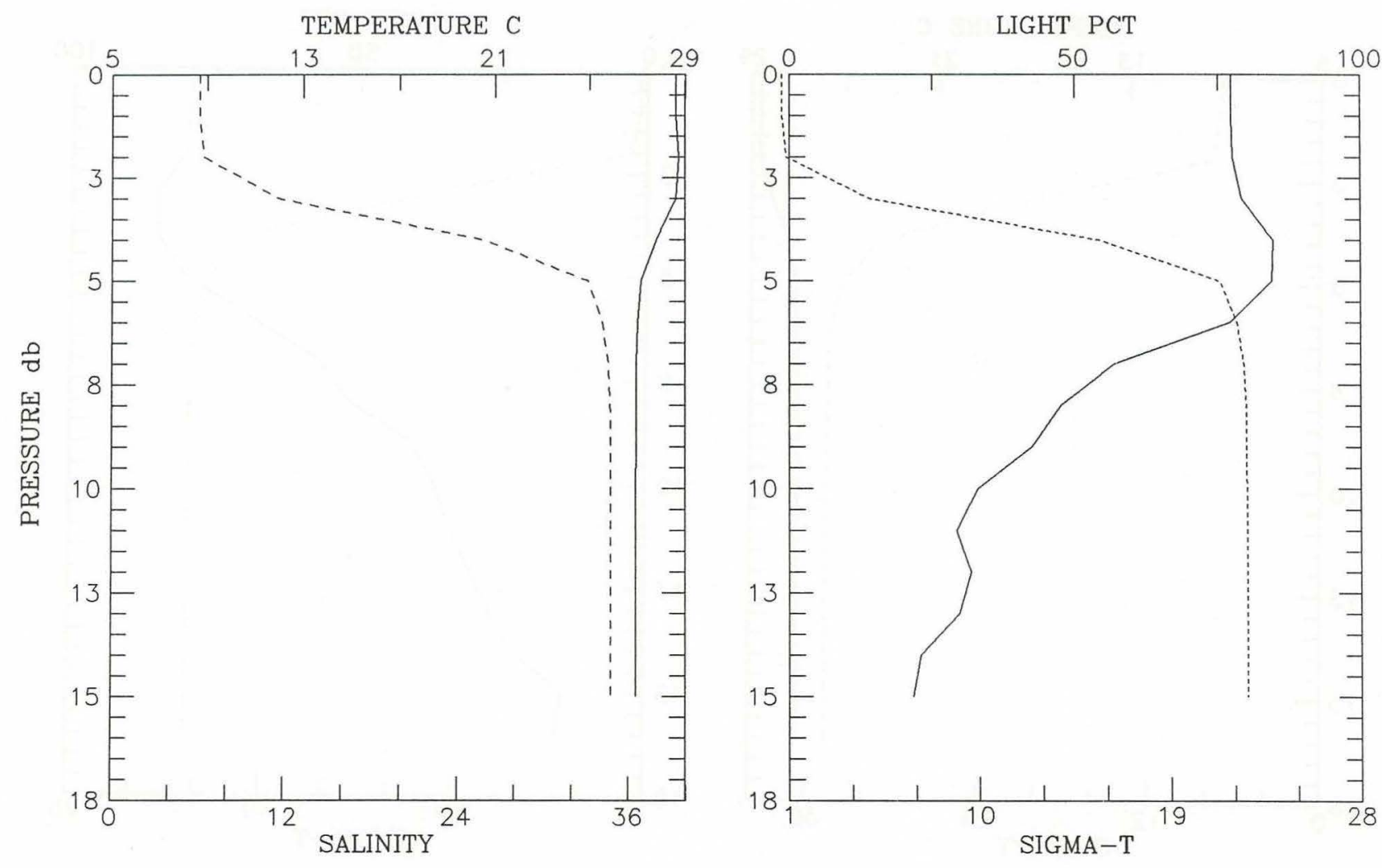


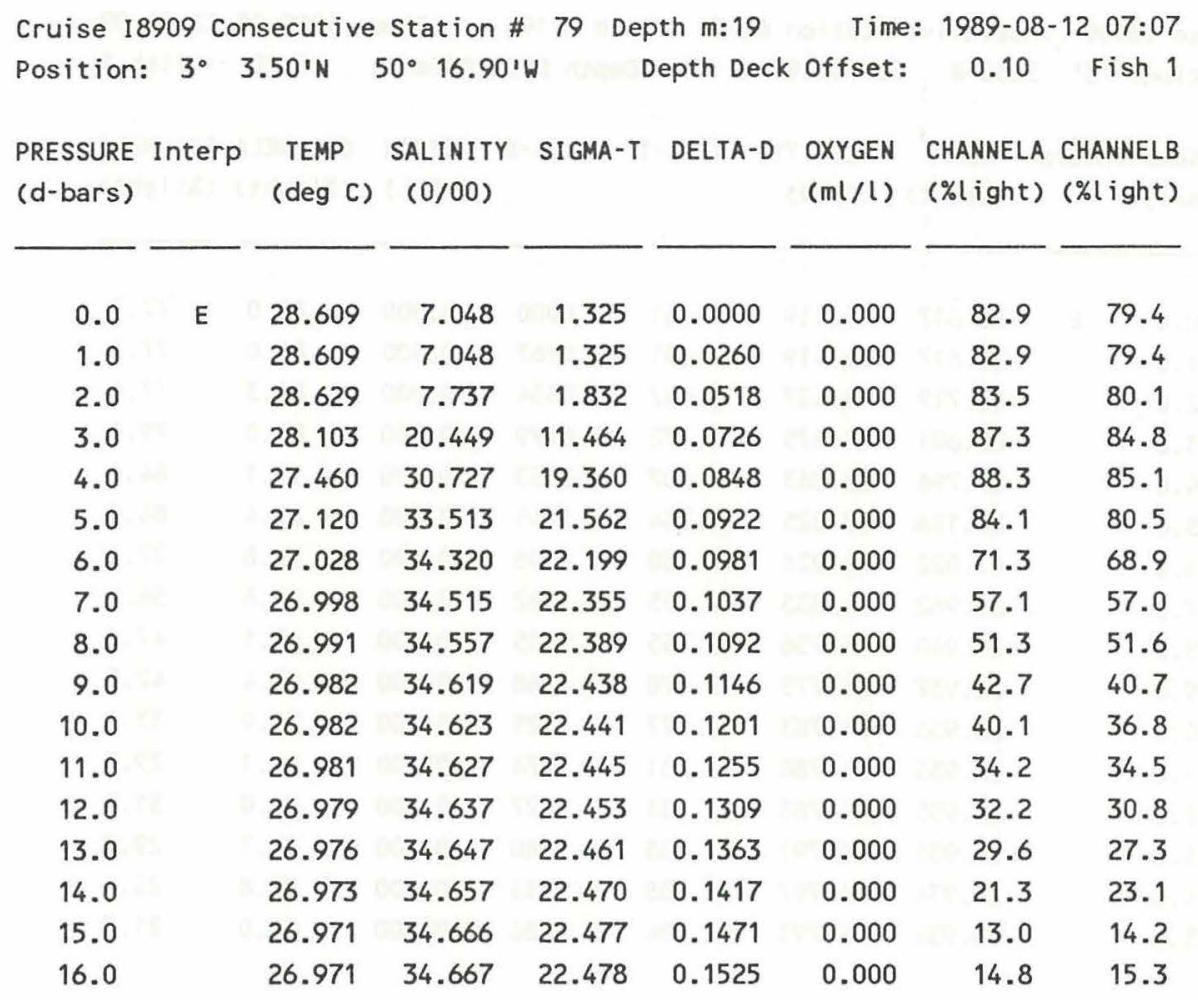
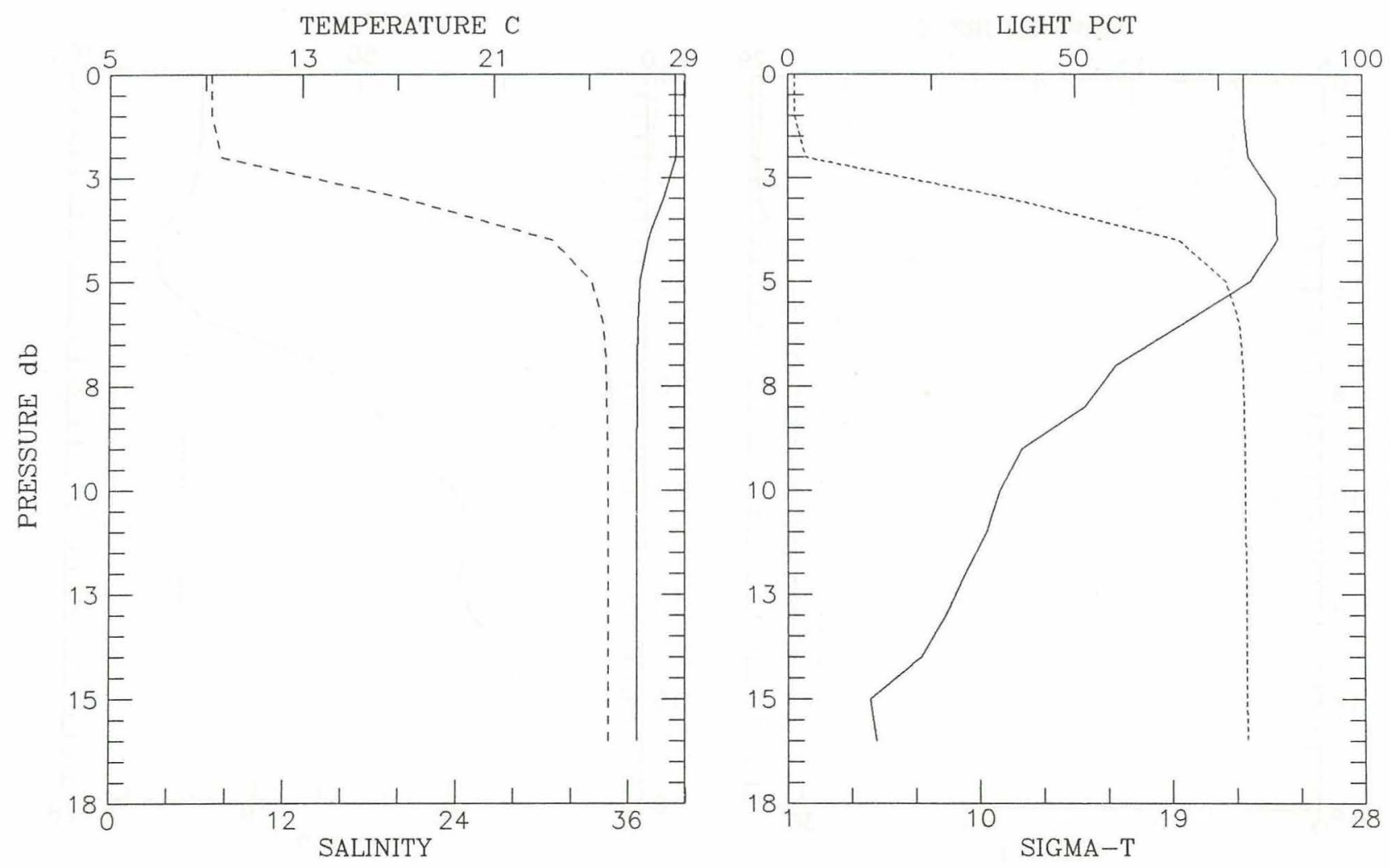


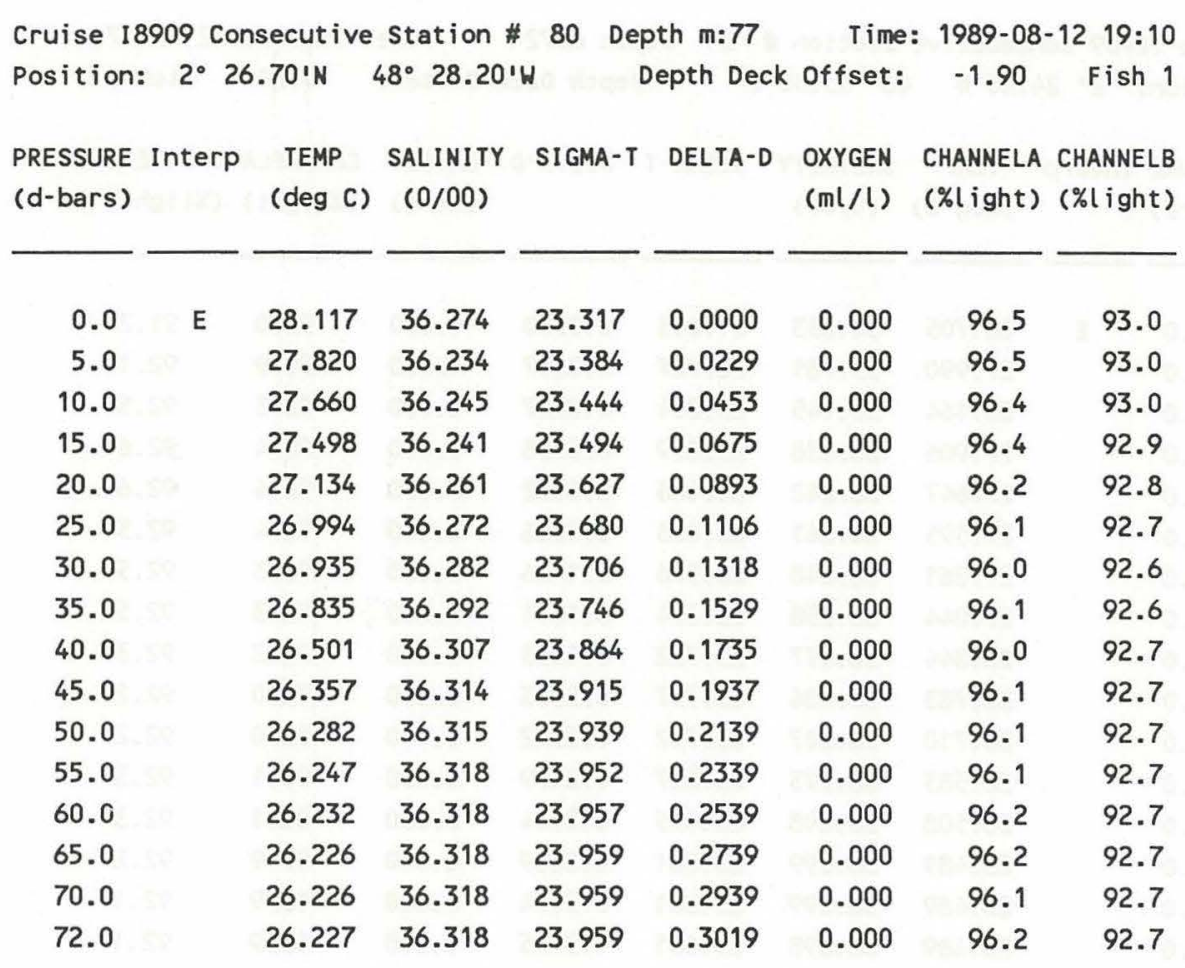
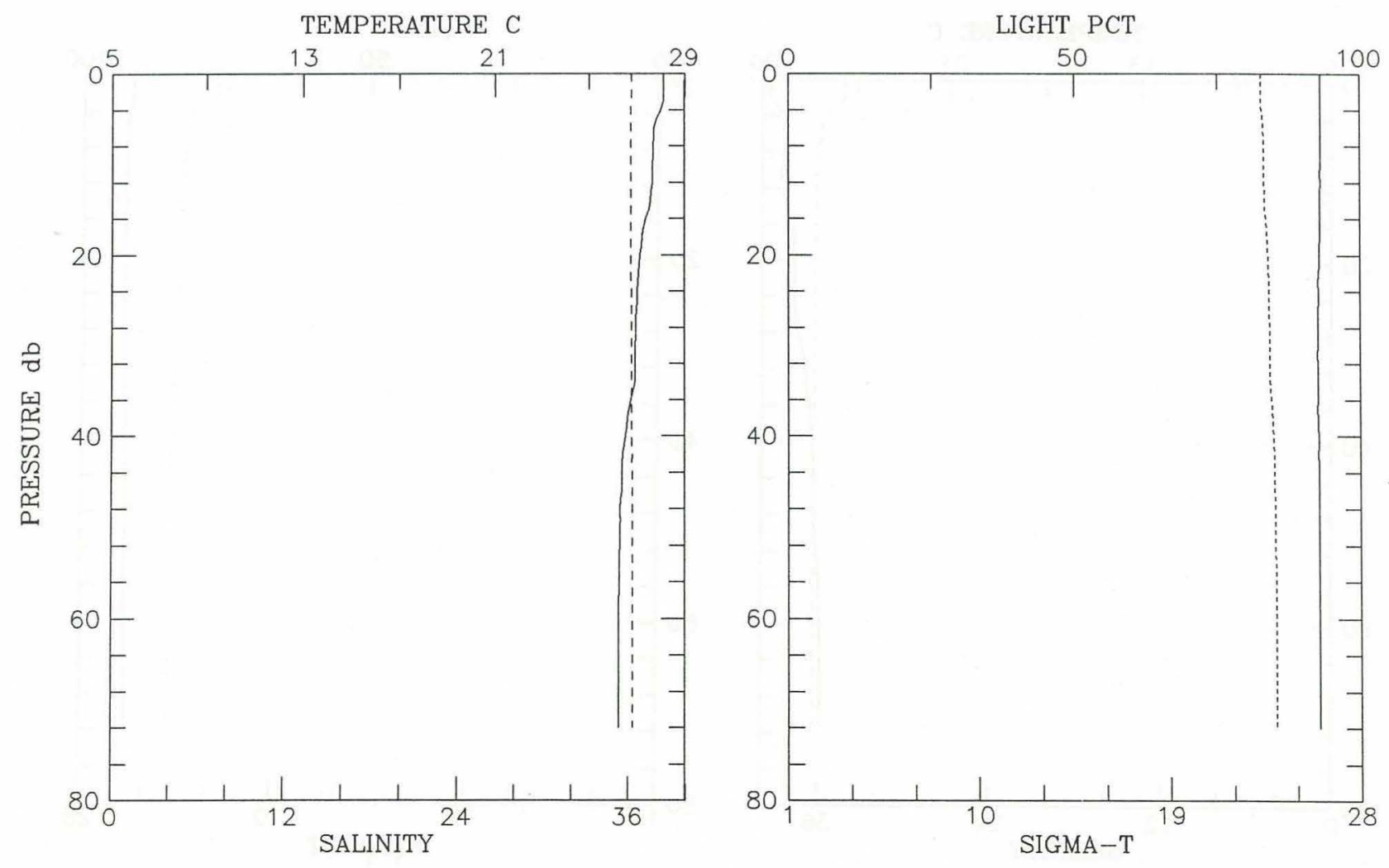


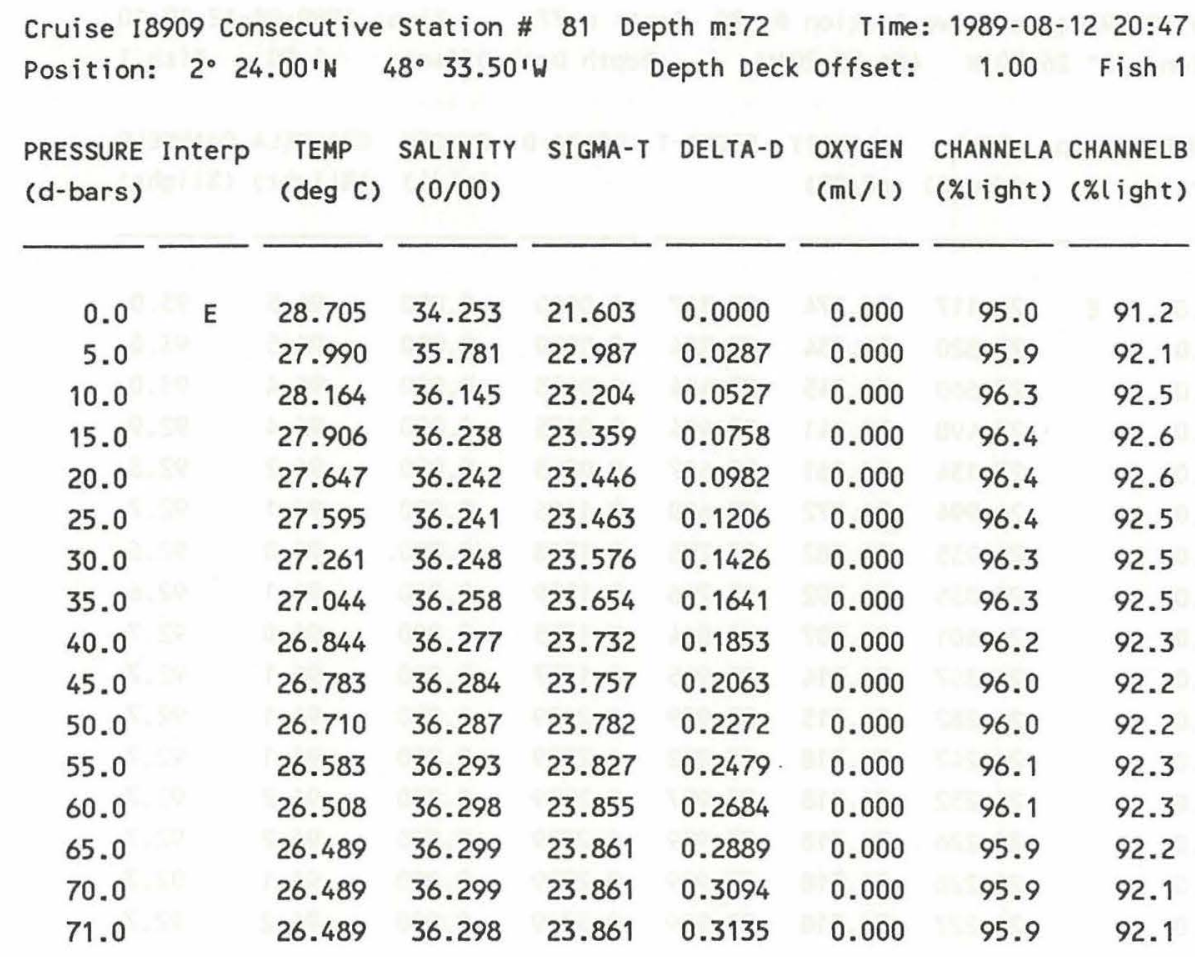
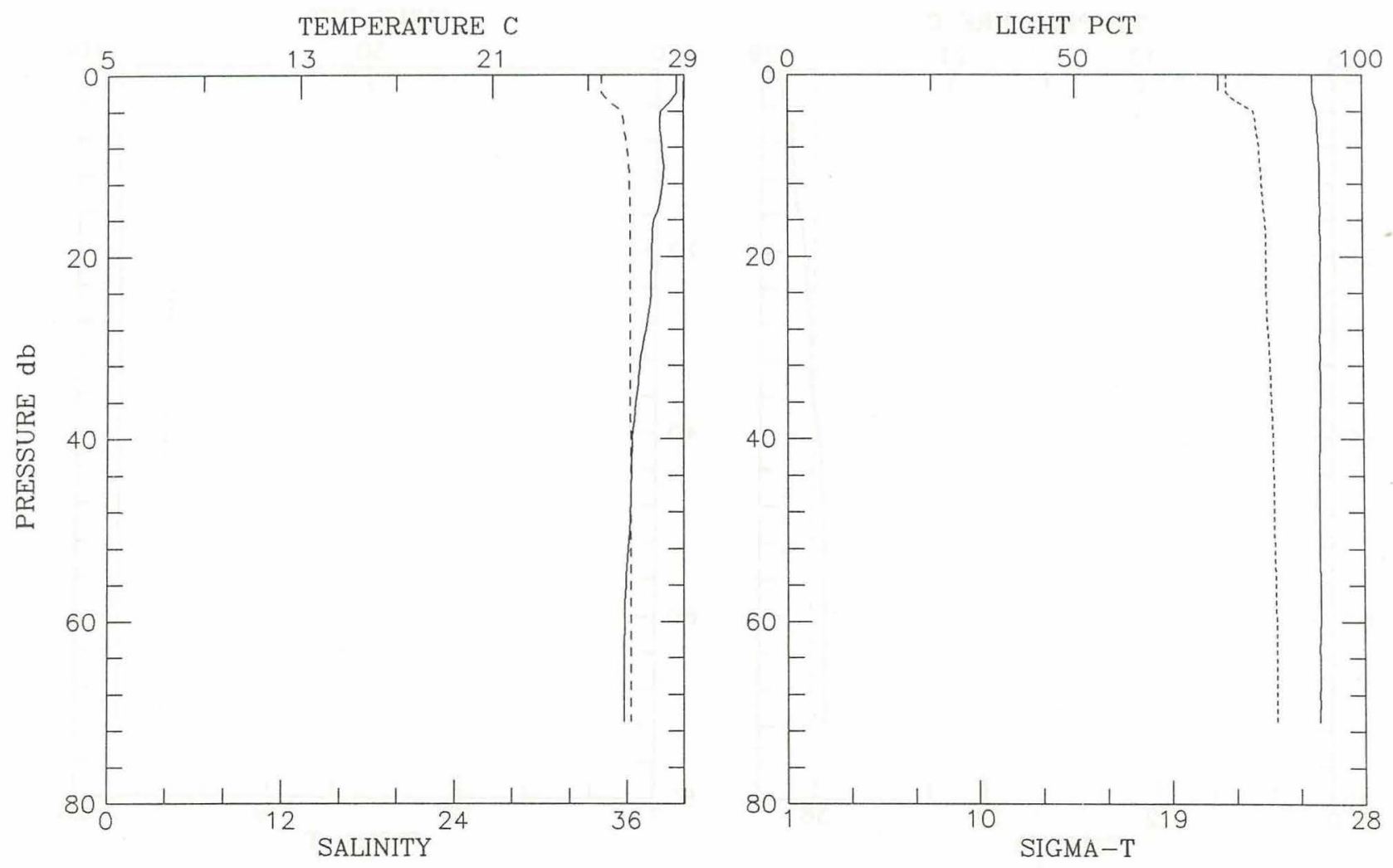


\begin{tabular}{|c|c|c|c|c|c|c|c|c|}
\hline \multirow{2}{*}{$\begin{array}{l}\text { Position: } \\
\text { PRESSURE } \\
\text { (d-bars) }\end{array}$} & \multicolumn{2}{|c|}{$2^{\circ} 16.40^{\prime} \mathrm{N}$} & \multicolumn{2}{|c|}{$48^{\circ} 37.90^{\prime} \mathrm{W}$} & Depth Deck & \multirow{2}{*}{$\begin{array}{l}\text { Offset: } \\
\text { OXYGEN } \\
(\mathrm{ml} / \mathrm{l})\end{array}$} & \multirow{2}{*}{$\begin{array}{r}-0.10 \\
\text { CHANNELA } \\
(\% \text { tight })\end{array}$} & \multirow{2}{*}{$\begin{array}{r}\text { Fish } 1 \\
\text { CHANNELB } \\
\text { (\%light) }\end{array}$} \\
\hline & Interp & $\begin{array}{c}\text { TEMP } \\
(\operatorname{deg} C)\end{array}$ & $\begin{array}{c}\text { SALINITY } \\
(0 / 00)\end{array}$ & SIGMA-T & DELTA-D & & & \\
\hline 0.0 & $E$ & 28.763 & 34.546 & 21.804 & 0.0000 & 0.000 & 95.4 & 91.1 \\
\hline 2.0 & & 28.291 & 35.336 & 22.554 & 0.0117 & 0.000 & 95.8 & 91.3 \\
\hline 4.0 & & 28.008 & 35.831 & 23.019 & 0.0218 & 0.000 & 96.4 & 92.0 \\
\hline 6.0 & & 27.927 & 36.092 & 23.242 & 0.0312 & 0.000 & 96.7 & 92.2 \\
\hline 8.0 & & 27.817 & 36.140 & 23.314 & 0.0405 & 0.000 & 96.6 & 92.1 \\
\hline 10.0 & & 27.757 & 36.170 & 23.356 & 0.0496 & 0.000 & 96.3 & 91.9 \\
\hline 12.0 & & 27.732 & 36.183 & 23.374 & 0.0587 & 0.000 & 96.2 & 91.7 \\
\hline 14.0 & & 27.716 & 36.191 & 23.385 & 0.0678 & 0.000 & 96.1 & 91.7 \\
\hline 16.0 & & 27.702 & 36.198 & 23.395 & 0.0768 & 0.000 & 96.2 & 91.7 \\
\hline 18.0 & & 27.691 & 36.207 & 23.406 & 0.0858 & 0.000 & 96.2 & 91.8 \\
\hline 20.0 & & 27.674 & 36.215 & 23.417 & 0.0948 & 0.000 & 96.2 & 91.9 \\
\hline 22.0 & & 27.666 & 36.219 & 23.423 & 0.1038 & 0.000 & 96.3 & 91.9 \\
\hline 24.0 & & 27.631 & 36.228 & 23.441 & 0.1128 & 0.000 & 96.4 & 92.0 \\
\hline 26.0 & & 27.608 & 36.236 & 23.454 & 0.1217 & 0.000 & 96.5 & 92.1 \\
\hline 28.0 & & 27.548 & 36.242 & 23.478 & 0.1306 & 0.000 & 96.6 & 92.1 \\
\hline 30.0 & & 27.468 & 36.241 & 23.504 & 0.1395 & 0.000 & 96.6 & 92.2 \\
\hline 32.0 & & 27.339 & 36.237 & 23.542 & 0.1483 & 0.000 & 96.7 & 92.3 \\
\hline 34.0 & & 26.850 & 36.234 & 23.698 & 0.1569 & 0.000 & 96.3 & 91.8 \\
\hline 36.0 & & 26.638 & 36.239 & 23.769 & 0.1653 & 0.000 & 95.7 & 91.3 \\
\hline 38.0 & & 26.620 & 36.240 & 23.776 & 0.1737 & 0.000 & 95.5 & 91.2 \\
\hline 40.0 & & 26.571 & 36.242 & 23.793 & 0.1820 & 0.000 & 95.3 & 91.0 \\
\hline
\end{tabular}
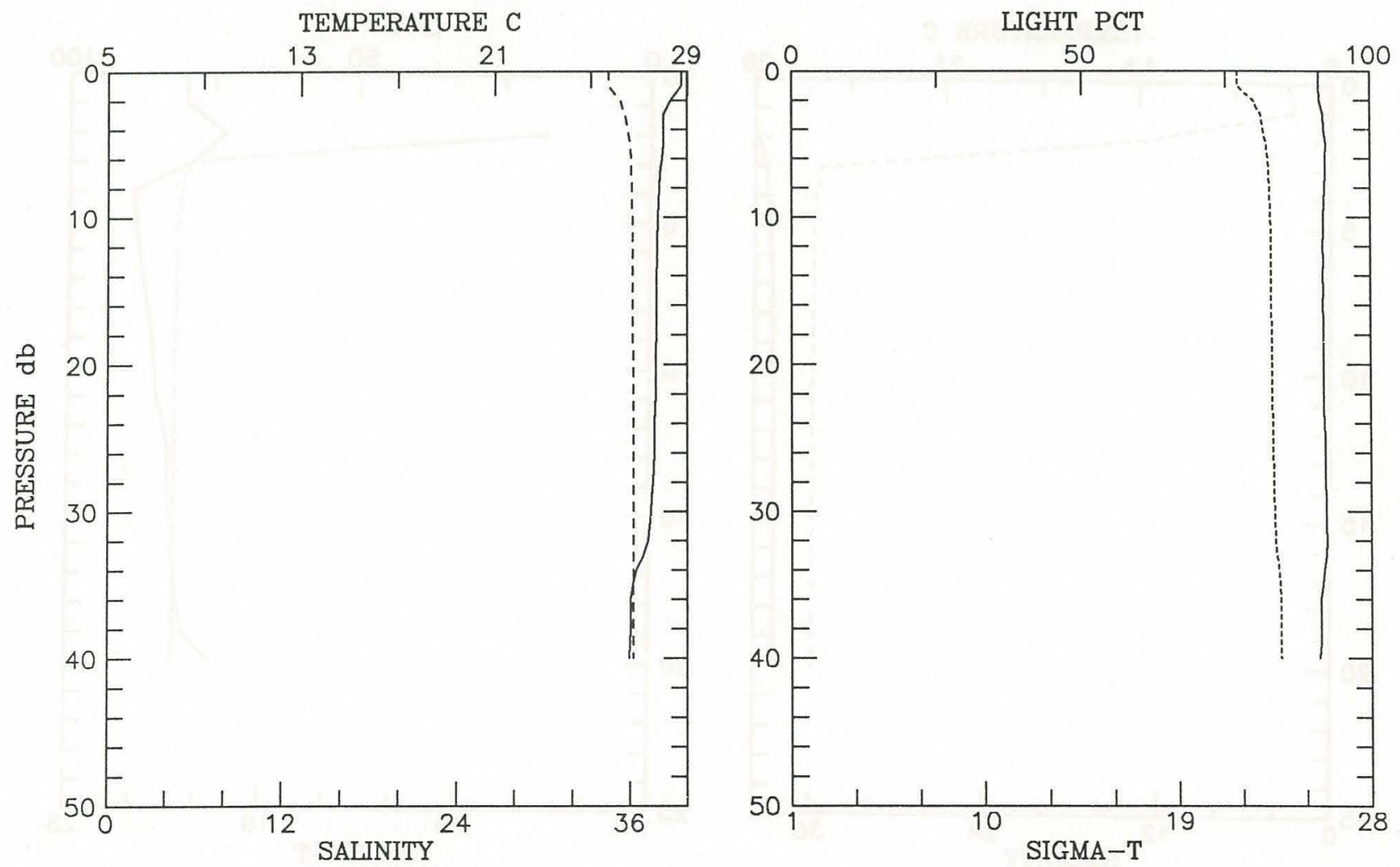


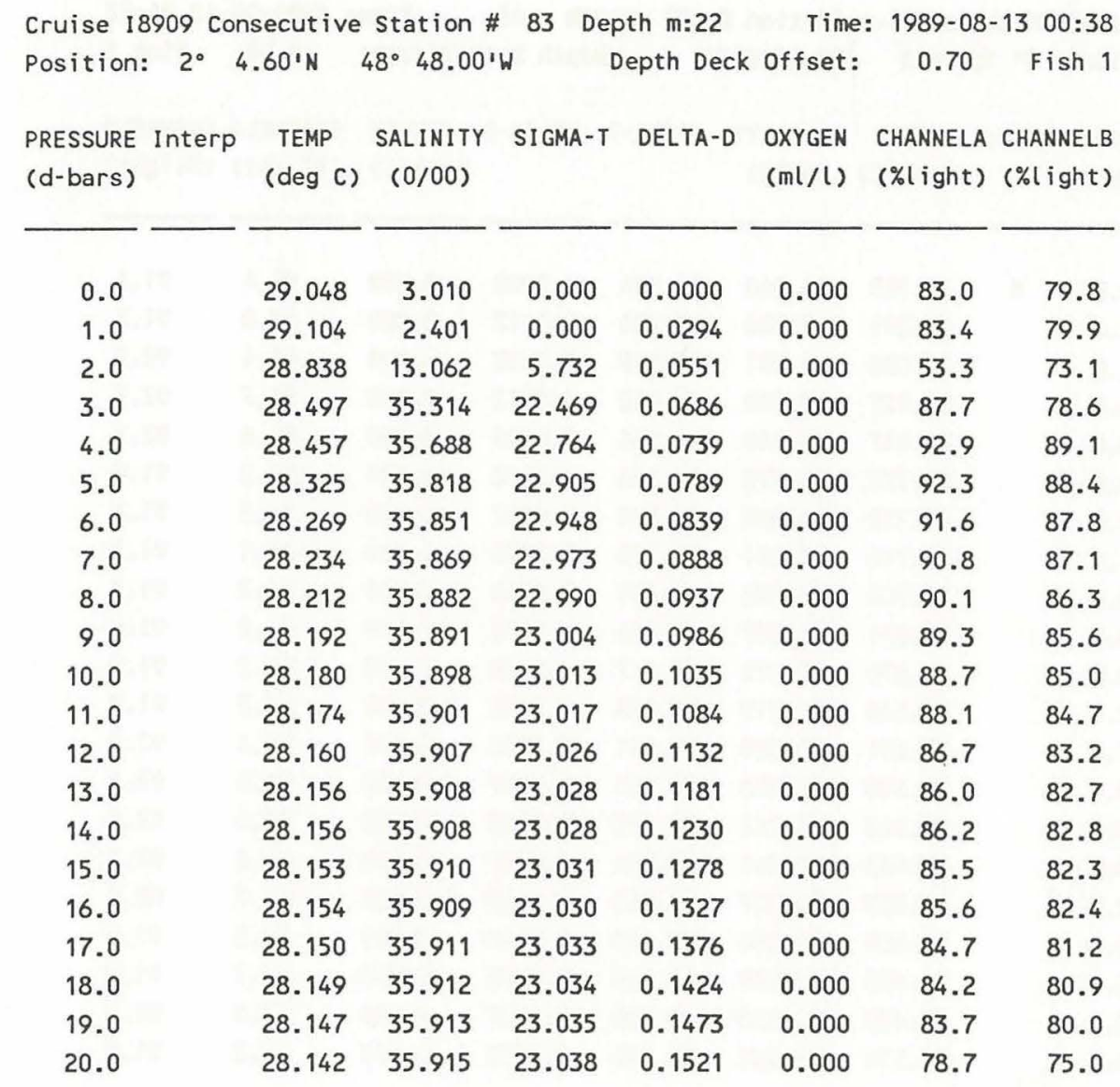
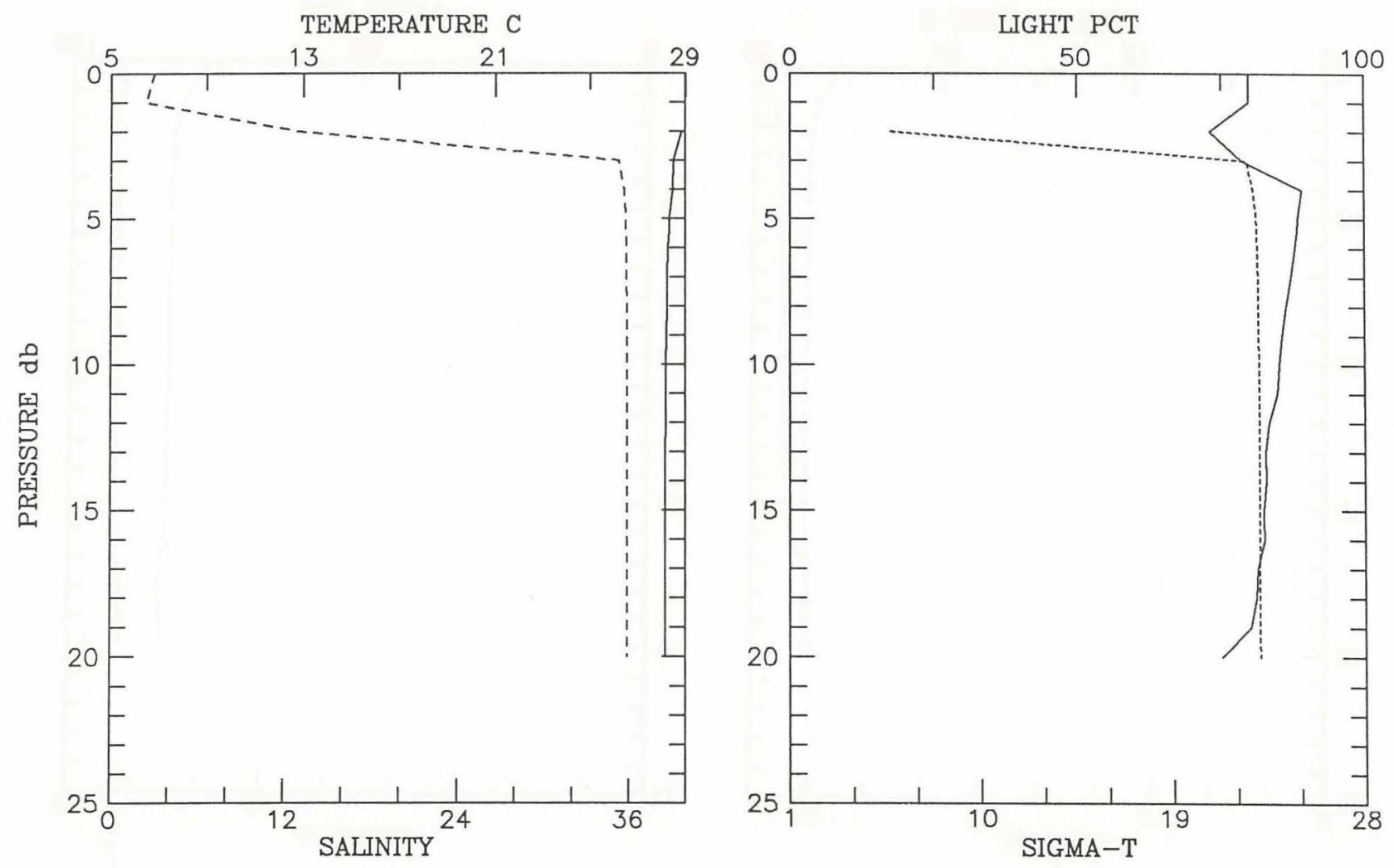


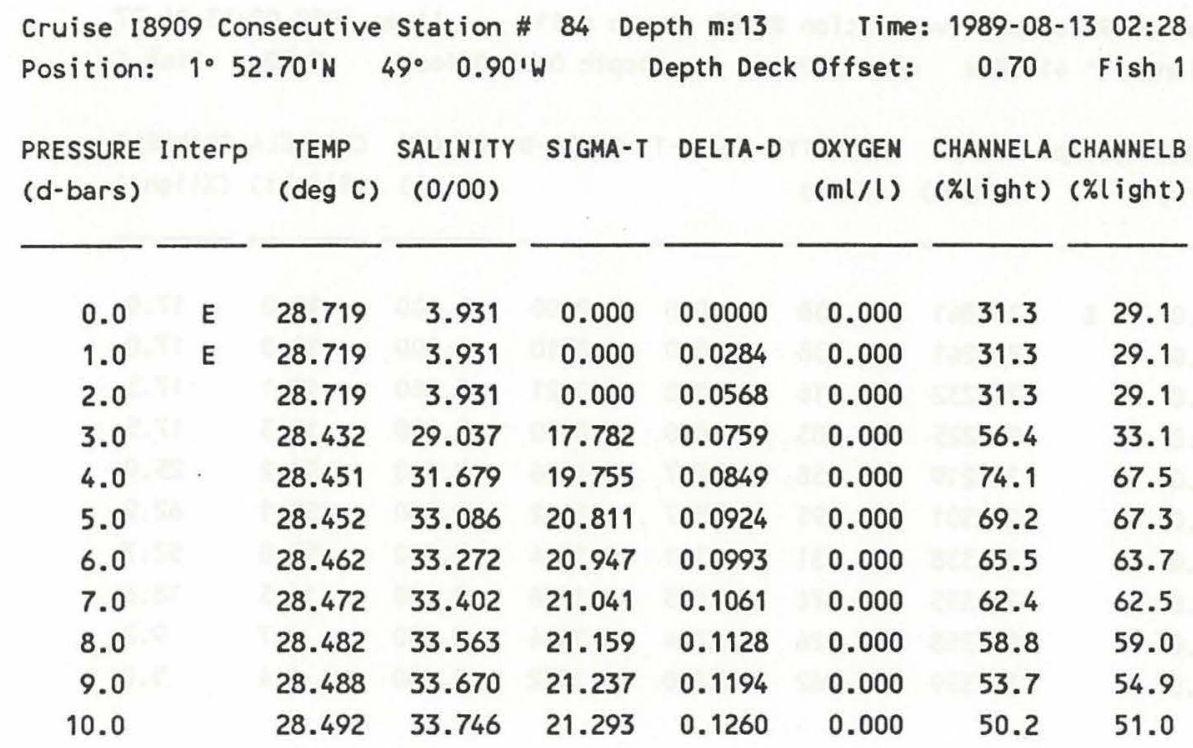
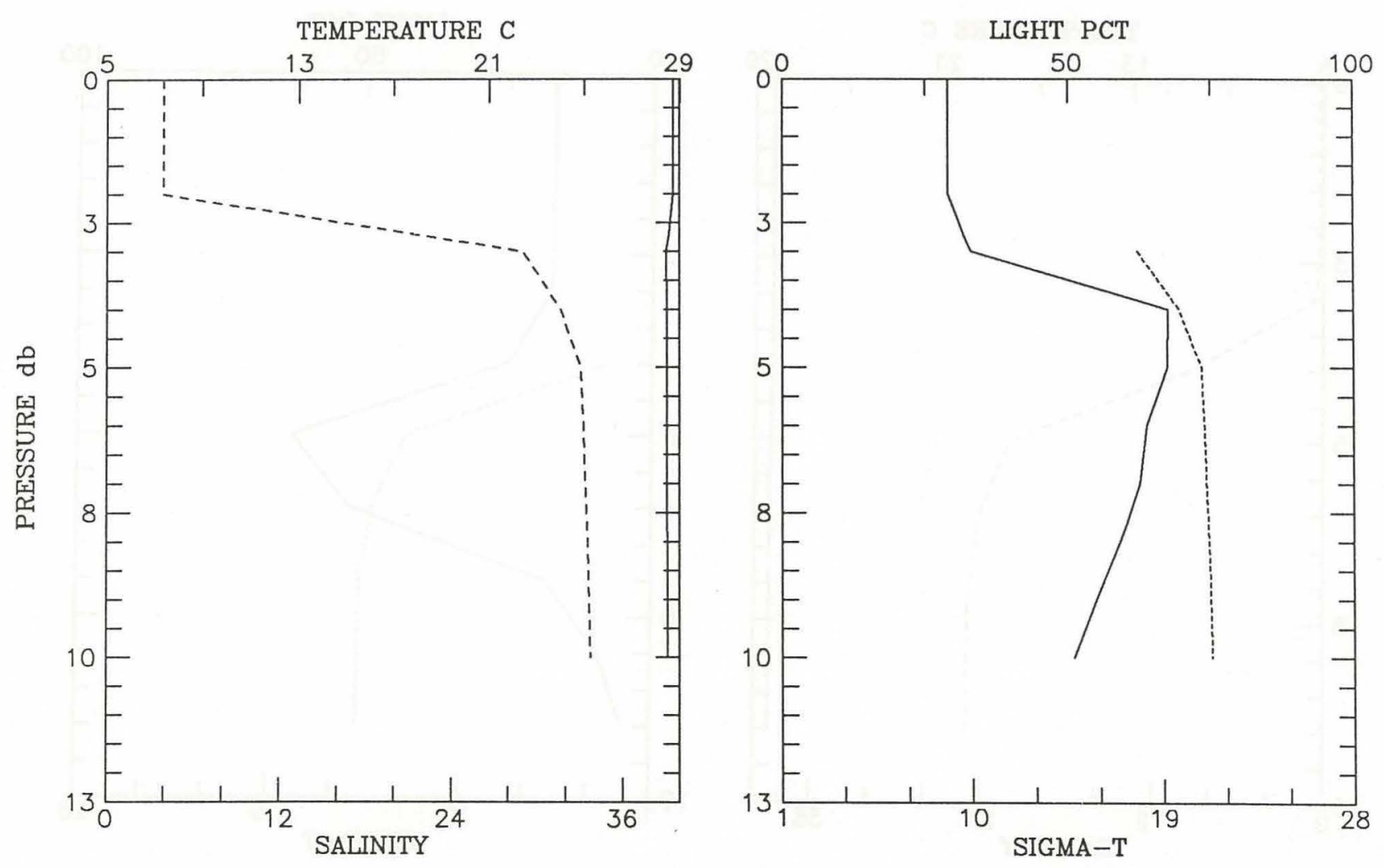


\begin{tabular}{|c|c|c|c|c|c|c|c|c|}
\hline Position: & $=\quad 1^{\circ} 41$ & $.00^{\prime} \mathrm{N}$ & $49^{\circ} 12.00$ & & epth Deck & Offset: & 0.70 & Fish 1 \\
\hline $\begin{array}{l}\text { PRESSURE } \\
\text { (d-bars) }\end{array}$ & Interp & $\begin{array}{c}\text { TEMP } \\
(\operatorname{deg} C)\end{array}$ & $\begin{array}{l}\text { SALINITY } \\
(0 / 00)\end{array}$ & SI GMA-T & DELTA-D & $\begin{array}{l}\text { OXYGEN } \\
(\mathrm{ml} / \mathrm{l})\end{array}$ & $\begin{array}{l}\text { CHANNELA } \\
\text { (\%light) }\end{array}$ & $\begin{array}{l}\text { CHANNELB } \\
\text { (\%light) }\end{array}$ \\
\hline 0.0 & $E$ & 28.241 & 0.238 & 0.000 & 0.0000 & 0.000 & 18.0 & 17.0 \\
\hline 1.0 & & 28.241 & 0.238 & 0.000 & 0.0310 & 0.000 & 18.0 & 17.0 \\
\hline 2.0 & & 28.232 & 0.216 & 0.000 & 0.0621 & 0.000 & 18.1 & 17.3 \\
\hline 3.0 & & 28.225 & 0.483 & 0.000 & 0.0930 & 0.000 & 19.3 & 17.5 \\
\hline 4.0 & & 28.219 & 9.258 & 3.087 & 0.1206 & 0.000 & 33.2 & 25.0 \\
\hline 5.0 & & 28.301 & 21.995 & 12.557 & 0.1402 & 0.000 & 55.1 & 62.9 \\
\hline 6.0 & & 28.338 & 24.131 & 14.141 & 0.1544 & 0.000 & 53.0 & 52.7 \\
\hline 7.0 & & 28.355 & 24.876 & 14.693 & 0.1676 & 0.000 & 16.5 & 18.6 \\
\hline 8.0 & & 28.358 & 25.026 & 14.804 & 0.1804 & 0.000 & 6.7 & 9.2 \\
\hline 9.0 & & 28.359 & 25.062 & 14.830 & 0.1932 & 0.000 & 4.4 & 5.0 \\
\hline
\end{tabular}
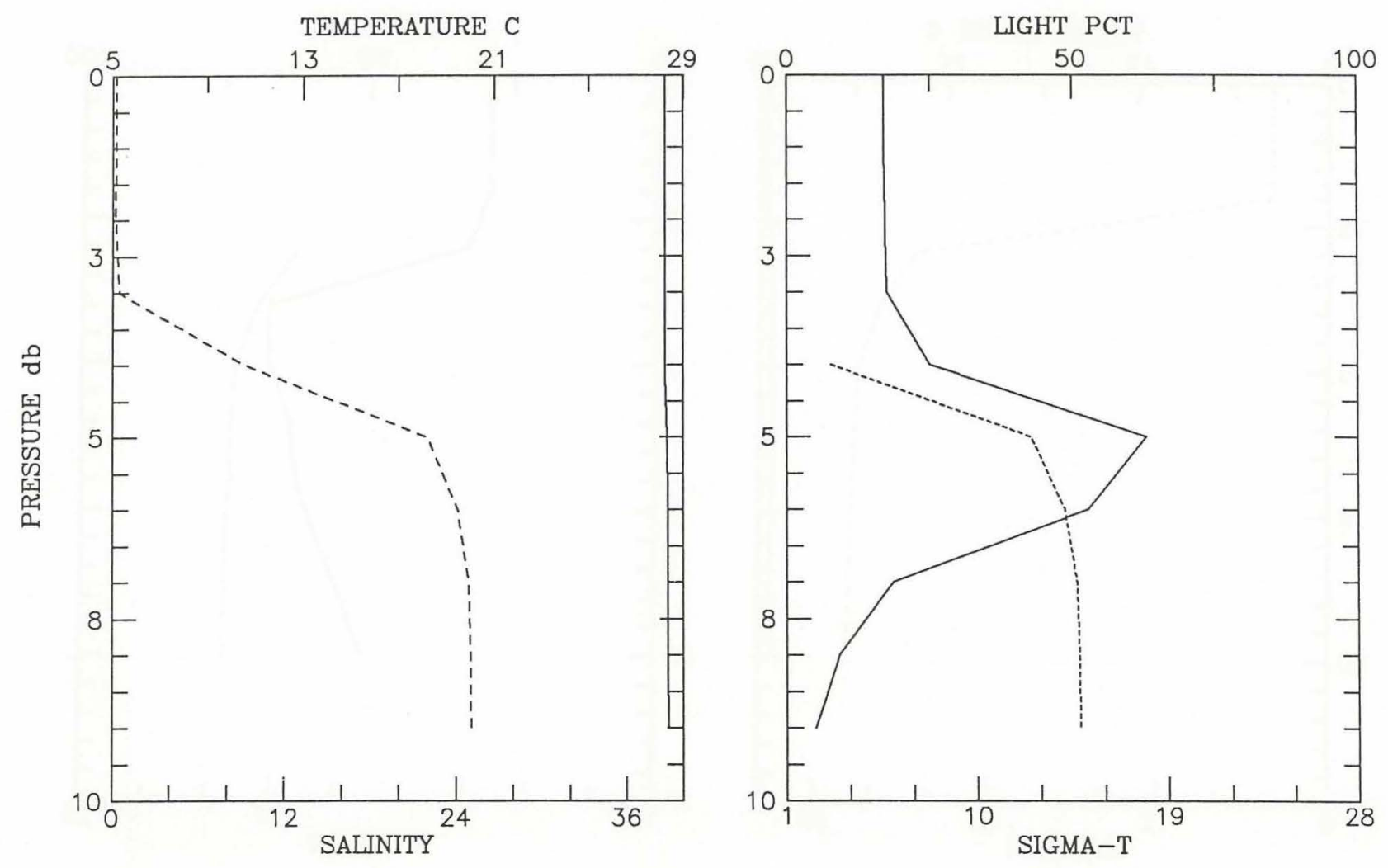


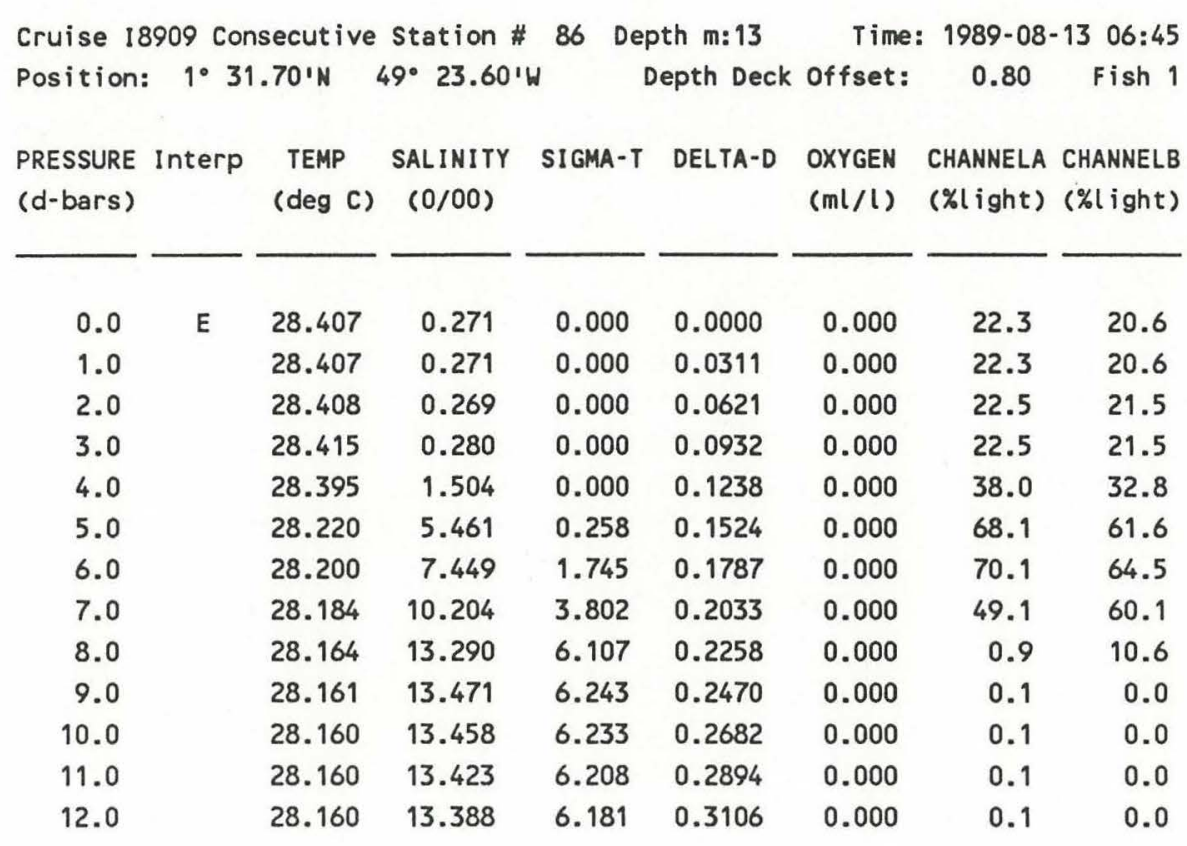
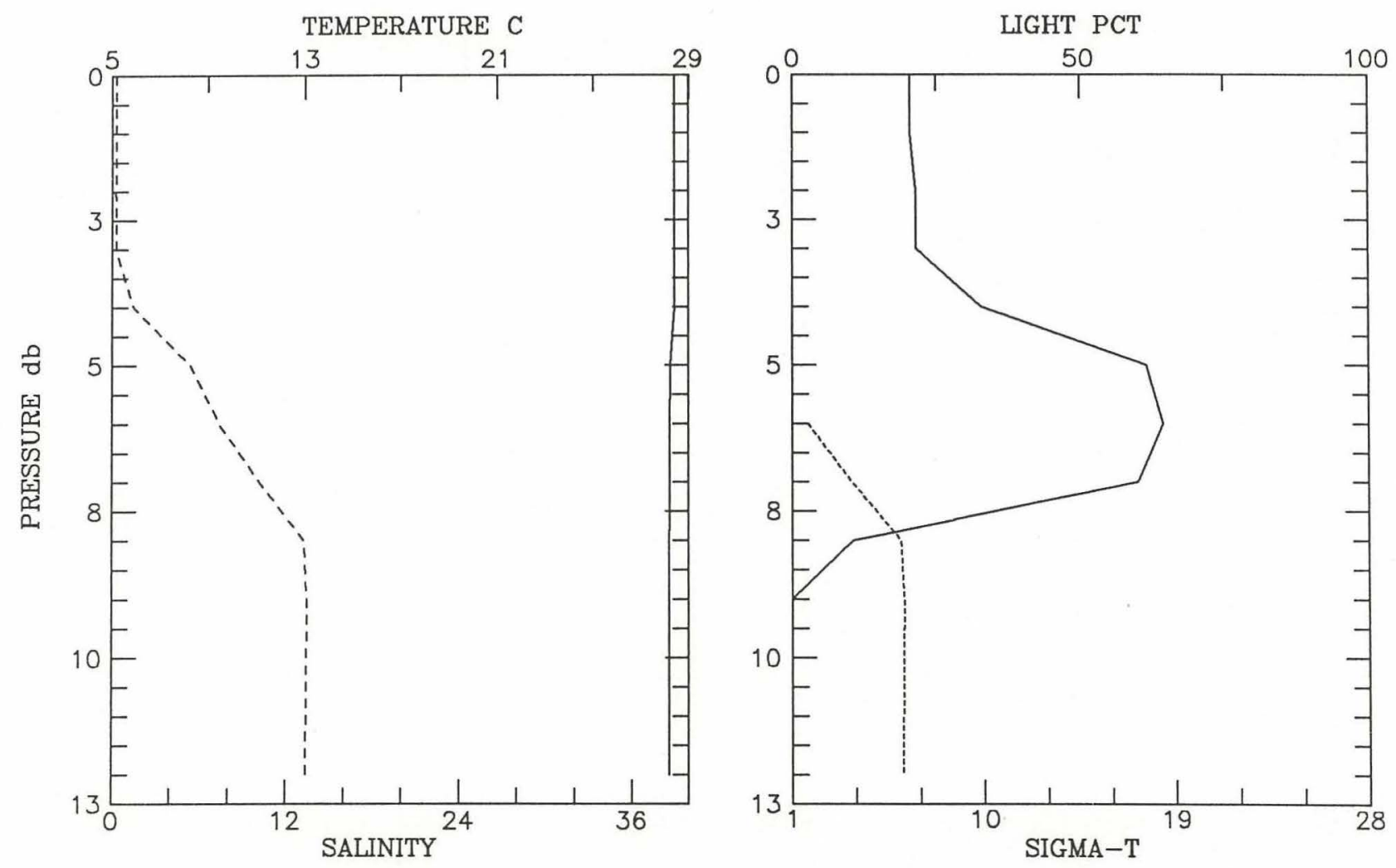


\begin{tabular}{|c|c|c|}
\hline $\begin{array}{l}\text { REPORT DOCUMENTATION } \\
\text { PAGE }\end{array}$ & $\begin{array}{l}\text { 1. REPORT NO. } \\
\text { WHOI-89-53 }\end{array}$ & 3. Recipient's Accession No. \\
\hline \multirow{2}{*}{\multicolumn{2}{|c|}{$\begin{array}{l}\text { 4. Title and Subtitle } \\
\text { CTD Obscrvations on the North Brazil Shelf During A Multidisciplinary Amazon Shelf } \\
\text { SEDiment Study, AMASSEDS, August } 1989 .\end{array}$}} & $\begin{array}{l}\text { 5. Report Date } \\
\text { November, } 1989\end{array}$ \\
\hline & & 6. \\
\hline \multicolumn{2}{|c|}{$\begin{array}{l}\text { 7. Author(s) } \\
\text { Richard Limeburner and Robert C. Beardsley }\end{array}$} & $\begin{array}{l}\text { 8. Performing Organization Rept. No. } \\
\text { WHOI-89-53 }\end{array}$ \\
\hline \multirow{2}{*}{\multicolumn{2}{|c|}{$\begin{array}{l}\text { 9. Performing Organization Name and Address } \\
\text { The Woods Hole Oceanographic Institution } \\
\text { Woods Hole, Massachusetts } 02543\end{array}$}} & 10. Project/Task/Work Unit No. \\
\hline & & $\begin{array}{l}\text { 11. Contract(C) or Grant(G) No. } \\
\text { (C) } \\
\text { (G) OCE } 88-12917\end{array}$ \\
\hline \multicolumn{2}{|c|}{ 12. Sponsoring Organization Name and Address } & $\begin{array}{l}\text { 13. Type of Report \& Period Covered } \\
\text { Technical Report }\end{array}$ \\
\hline \multicolumn{2}{|c|}{ Funding was provided by the National Science Foundation } & 14. \\
\hline
\end{tabular}

15. Supplementary Notes

This report should be cited as: Woods Hole Oceanog. Inst. Tech. Rept., WHOI-89-53.

16. Abstract (Limit: 200 words)

CTD and acoustic Doppler current profiler (ADCP) observations were made on the North Brazil shelf adjacent to the mouth of the Amazon River during R/V Iselin cruise I8909 August 3-14, 1989 as part of A Multidisciplinary Amazon Shelf SEDiment Study (AMASSEDS). These observations were obtained during a large-scale survey in support of geological and geochemical sampling, an anchored time series station consisting of 26 hourly CTD casts, and one transect which was repeated off the mouth of the Amazon River. The maximum sampling depth at each station was within two meters of the bottom.

The primary objectives of the AMASSEDS hydrographic measurement program were to (a) observe and characterize the temperature, salinity, density, oxygen, fluorescence and light transmission fields and their spatial variability on the north Brazilian shelf directly influenced by the Amazon River discharge, (b) resolve the seaward extent and vertical structure of the surface plume of low salinity Amazon River water during different stages of river discharge, (c) describe the spatial structure of the turbidity and associated suspended sediment distributions across the shelf, (d) characterize the properties of the Amazon shelf water beneath the surface plume and their seasonal variability, and (e) describe the landward penetration of the North Brazil Current (NBC) with respect to water properties and shelf currents. This report represents a summary in graphic and tabular form of the hydrographic observations made during the first AMASSEDS cruise (I8909) on the R/V Iselin.

17. Document Analysis a. Descriptors

1. hydrography/CTD

2. North Brazil coastal region

3. Amazon river

4. AMASSEDS (A Multidisciplinary Amazon Shelf SEDiment Study)

b. Identifiers/Open-Ended Terms

c. COSATI Field/Group

18. Availability Statement

Approved for publication; distribution unlimited.

\begin{tabular}{|l|l|}
\hline $\begin{array}{c}\text { 19. Security Class (This Report) } \\
\text { UNCLASSIFIED }\end{array}$ & $\begin{array}{c}\text { 21. No. of Pages } \\
134\end{array}$ \\
\hline 20. Security Class (This Page) & 22. Price \\
\hline
\end{tabular}




\section{DOCUMENT LIBRARY}

July 5, 1989

Distribution List for Technical Report Exchange

Attn: Stella Sanchez-Wade

Documents Section

Scripps Institution of Oceanography

Library, Mail Code C-075C

La Jolla, CA 92093

Hancock Library of Biology \& Oceanography

Alan Hancock Laboratory

University of Southern California

University Park

Los Angeles, CA 90089-0371

Gifts \& Exchanges

Library

Bedford Institute of Oceanography

P.O. Box 1006

Dartmouth, NS, B2Y 4A2, CANADA

Office of the International

Ice Patrol

c/o Coast Guard R \& D Center

Avery Point

Groton, CT 06340

Library

Physical Oceanographic Laboratory

Nova University

8000 N. Ocean Drive

Dania, FL 33304

NOAA/EDIS Miami Library Center 4301 Rickenbacker Causeway

Miami, FL 33149

Library

Skidaway Institute of Oceanography

P.O. Box 13687

Savannah, GA 31416

Institute of Geophysics

University of Hawaii

Library Room 252

2525 Correa Road

Honolulu, HI 96822

Library

Chesapeake Bay Institute

4800 Atwell Road

Shady Side, MD 20876

MIT Libraries

Serial Journal Room 14E-210

Cambridge, MA 02139
Director, Ralph M. Parsons Laboratory

Room 48-311

MIT

Cambridge, MA 02139

Marine Resources Information Center

Building E38-320

MIT

Cambridge, MA 02139

Library

Lamont-Doherty Geological Observatory

Colombia University

Palisades, NY 10964

Library

Serials Department

Oregon State University

Corvallis, OR 97331

Pell Marine Science Library

University of Rhode Island

Narragansett Bay Campus

Narragansett, RI 02882

Working Collection

Texas A\&M University

Dept. of Oceanography

College Station, TX 77843

Library

Virginia Institute of Marine Science

Gloucester Point, VA 23062

Fisheries-Oceanography Library

151 Oceanography Teaching Bldg.

University of Washington

Seattle, WA 98195

Library

R.S.M.A.S.

University of Miami

4600 Rickenbacker Causeway

Miami, FL 33149

Maury Oceanographic Library

Naval Oceanographic Office

Bay St. Louis

NSTL, MS 39522-5001

Marine Sciences Collection

Mayaguez Campus Library

University of Puerto Rico

Mayagues, Puerto Rico 00708 